\title{
Total Synthesis of (-)-Disorazole $\mathbf{C}_{\mathbf{1}}$
}

Luca Lizzadro, Oliver Spieß, and Dieter Schinzer*.

Chemisches Institut

Otto-von-Guericke-Universität

Universitätsplatz 2, 39106 Magdeburg (Germany)

\section{SUPPORTING INFORMATION}

\section{Table of Contents}

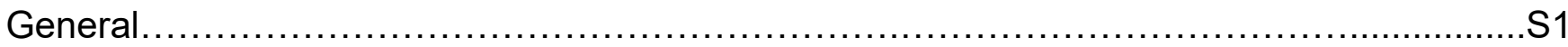

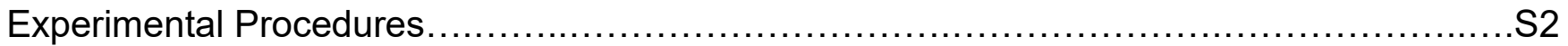

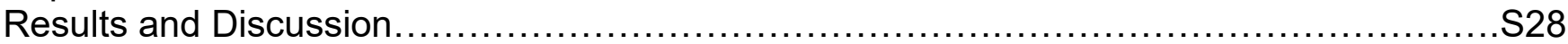

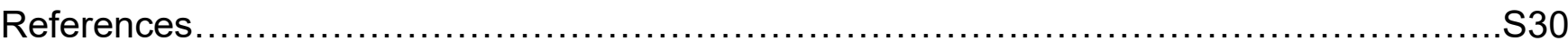

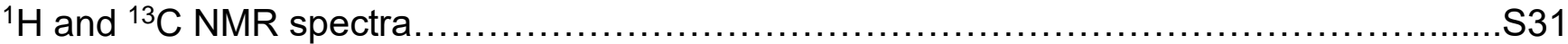

\section{General}

Solvents were dried by standard procedures and redistilled under $\mathrm{N}_{2}$ atmosphere prior to use. All reactions were run under nitrogen unless otherwise stated. For reactions that require heating, an oil bath was used. The products were purified by flash chromatography on Merck silica gel $60(40-63 \mu \mathrm{m})$. Electrospray (ESI) and electron ionisation (EI) mass spectra were recorded on Finnigan MAT 95 and Waters Xevo G2-TOF spectrometers. ${ }^{1} \mathrm{H}$ and ${ }^{13} \mathrm{C}$ NMR spectra were recorded on Brucker AVIII 400 and Brucker AVI 600 spectrometers. Chemical shifts $(\delta)$ are reported in ppm from tetramethylsilane, referenced to the solvent resonance resulting from incomplete deuteration $\left({ }^{1} \mathrm{H} \mathrm{NMR}=\mathrm{CDCl}_{3}: \delta 7.26, \mathrm{CD}_{3} \mathrm{OD}: \delta 3.31 ;{ }^{13} \mathrm{C} \mathrm{NMR}=\mathrm{CDCl}_{3}: \delta 77.16\right.$, $\mathrm{CD}_{3} \mathrm{OD}$ : $\delta$ 49.00). Data are reported as follows: chemical shift, multiplicity ( $\mathrm{s}=$ singlet, $d=$ doublet, $\mathrm{t}=$ triplet, $\mathrm{br}=$ broad, $\mathrm{m}=$ multiplet, $\mathrm{app}=$ apparent), coupling constants $(\mathrm{Hz})$ and integration. Optical rotations were recorded on Perkin-Elmer 341 and Anton Paar MCP150 polarimeters. Infrared (IR) spectra were recorded on Brucker Vertex 70v Bands are characterized as strong (s), medium (m), weak (w) or broad (br). 


\section{Experimental Procedures}

(S)-HYTRA 2 and (E)-2,2-dimethyl-3-pentenal $\mathbf{3}$ are both commercially available, but the authors generally synthesized them using the following procedures.

\section{Synthesis of (S)-(-)-2-Hydroxy-1,2,2-triphenylethylacetate [(S)-HYTRA] (2)}

(S)-HYTRA 2 was prepared according to the procedure reported by Braun et al.[1] starting from (S)-(+)-Mandelic acid and with some modifications in the last step. The use of acetic anhydride in the presence of catalytic scandium triflate allowed a much easier work up, providing the same yield as the method used by Braun with acetyl chloride and pyridine.

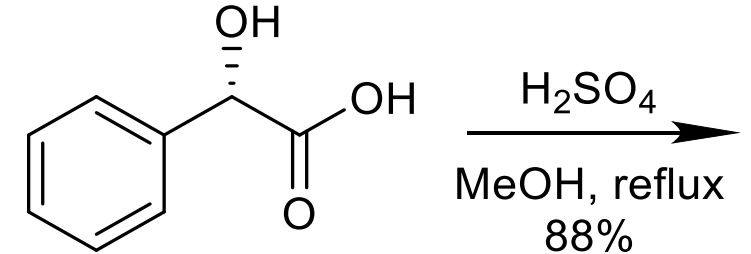

$88 \%$<smiles>COC(=O)[C@H](O)c1ccccc1</smiles>

S1

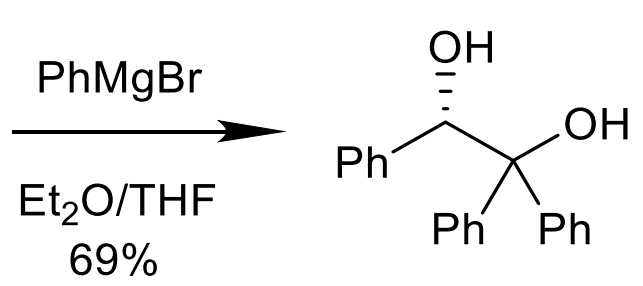

S2

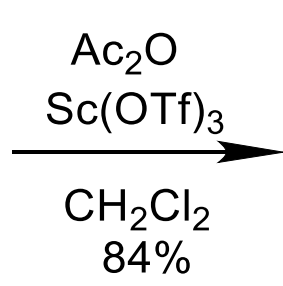

$84 \%$<smiles>CC(=O)O[C@@H](c1ccccc1)C(O)(c1ccccc1)c1ccccc1</smiles>

2

(S)-(+)-Mandelic acid methyl ester (S1)<smiles>COC(=O)[C@H](O)c1ccccc1</smiles>

\section{S1}

To a solution of (S)-(+)-Mandelic acid (57.13 g, $376 \mathrm{mmol}, 1 \mathrm{eq})$ in $\mathrm{MeOH}(300 \mathrm{~mL})$, concentrated sulfuric acid $(600 \mu \mathrm{L}, 11.3 \mathrm{mmol}, 0.03 \mathrm{eq})$ was added and the mixture was refluxed for $4 \mathrm{~h}$. The reaction was quenched with $\mathrm{K}_{2} \mathrm{CO}_{3}(1.04 \mathrm{~g}, 7.52 \mathrm{mmol}, 0.02 \mathrm{eq})$ in 1.2 $\mathrm{mL}$ of water and the $\mathrm{MeOH}$ was evaporated in vacuo. Then $\mathrm{Et}_{2} \mathrm{O}(300 \mathrm{~mL})$ was added and the solid was filtered off; the filtrate was concentrated and crystallized from hexane $(75 \mathrm{~mL})$ to furnish ester $\mathbf{S 1}(54.9 \mathrm{~g}, 330.72 \mathrm{mmol}, 88 \%)$ as a white solid.

General Data: $\mathrm{C}_{9} \mathrm{H}_{10} \mathrm{O}_{3}$; FW: 166.06; TLC: $\mathrm{R}_{\mathrm{f}}=0.35$ (pentane/Et2O 1:1); UV (+); Vanillin: yellow; mp: $56-58^{\circ} \mathrm{C} ;[\alpha]_{D}^{20}=+144.0(c=1.0, \mathrm{MeOH})$.

${ }^{1} \mathrm{H}-\mathrm{NMR}\left(600 \mathrm{MHz}^{\mathrm{CDCl}}{ }_{3}\right)$ : $\delta$ (ppm): 7.43-7.40 (m, 2H, Ar-H); 7.39-7.35 (m, 2H, Ar-H); 7.35$7.31(\mathrm{~m}, 1 \mathrm{H}, \mathrm{Ar}-\mathrm{H}) ; 5.18(\mathrm{~s}, 1 \mathrm{H}, \mathrm{CHOH}), 3.76\left(\mathrm{~s}, 3 \mathrm{H}, \mathrm{OCH}_{3}\right)$.

${ }^{13}$ C-NMR (151 MHz, CDCl $)$ : $\delta(\mathrm{ppm}): 174.3(\mathrm{C}=\mathrm{O}) ; 138.3(\mathrm{ArC}) ; 128.8(\mathrm{ArCH}) ; 128.7$ $(\mathrm{ArCH}) ; 126.7(\mathrm{ArCH}) ; 73.00(\mathrm{CH}) ; 53.2\left(\mathrm{OCH}_{3}\right)$. 
MS (ESI): $m / z(\%): 184.09(100)\left[M+\mathrm{NH}_{4}\right]^{+}, 189.05(87)[M+\mathrm{Na}]^{+}, 149.06(72)$. HRMS (ESI) m/ $z$ : [M+Na] ${ }^{+}$Calcd for $\mathrm{C}_{9} \mathrm{H}_{10} \mathrm{O}_{3} \mathrm{Na}: 189.0528$; found: 189.0538 .

(S)-(-)-1,1,2-Triphenyl-1,2-ethandiol (S2)<smiles>O[C@H](c1ccccc1)C(O)(c1ccccc1)c1ccccc1</smiles>

S2

To a solution of Phenylmagnesium bromide $3 \mathrm{M}$ in $\mathrm{Et}_{2} \mathrm{O}(200 \mathrm{~mL}, 600 \mathrm{mmol}, 5.0 \mathrm{eq})$, ester S1 $(20 \mathrm{~g}, 120.36 \mathrm{mmol}, 1 \mathrm{eq})$ in $\mathrm{Et}_{2} \mathrm{O}(120 \mathrm{~mL})$ and THF $(12 \mathrm{~mL})$ was added dropwise at $0^{\circ} \mathrm{C}$ at such a rate that the temperature does not rise above $10^{\circ} \mathrm{C}(90 \mathrm{~min}$ required). The mixture was allowed to slowly warm to room temperature overnight and then refluxed for 1 h. After cooling to room temperature, the mixture was carefully poured into ice $(200 \mathrm{~g})$ and $2 \mathrm{M} \mathrm{HCl}$ was added dropwise to adjust the $\mathrm{pH}$ value to 4 . The mixture was stirred for $1 \mathrm{~h}$ at room temperature and then the organic layer was separated. The aqueous phase was extracted with $\mathrm{CH}_{2} \mathrm{Cl}_{2}(3 \times 200 \mathrm{~mL})$ and the combined organic extracts were washed with $\mathrm{NaHCO}_{3}$, dried over $\mathrm{Na}_{2} \mathrm{SO}_{4}$, filtered and concentrated in vacuo. Crystallization of the residue from methanol $(70 \mathrm{~mL})$ afforded diol $\mathbf{S 2}(24.1 \mathrm{~g}, 83 \mathrm{mmol}, 69 \%)$ as a white needleshaped solid, which was dried under vacuum for several hours to remove all traces of methanol.

General Data: $\mathrm{C}_{20} \mathrm{H}_{18} \mathrm{O}_{2}$; FW: 290.13; TLC: $\mathrm{R}_{\mathrm{f}}=0.25$ (pentane/Et $2 \mathrm{O} 2: 1$ ); UV (+); mp: 123$127^{\circ} \mathrm{C} ;[\alpha]_{D}^{20}=-125.5\left(c=1.0, \mathrm{CHCl}_{3}\right)$.

${ }^{1} \mathrm{H}-\mathrm{NMR}(400 \mathrm{MHz}, \mathrm{CDCl})$ ): $\delta(\mathrm{ppm})$ : 7.73-7.64 (m, 2H, Ar- $H$ ); 7.46-7.36 (m, 2H, Ar- H); 7.35$7.28(\mathrm{~m}, 1 \mathrm{H}, \mathrm{Ar}-\mathrm{H})$; 7.20-7.02 (m, 10H, Ar-H); $5.61(\mathrm{~s}, 1 \mathrm{H}, \mathrm{CHOH}) ; 3.15\left(\mathrm{~s}, 1 \mathrm{H}, \mathrm{Ph}_{2} \mathrm{COH}\right)$; $2.47(\mathrm{~s}, 1 \mathrm{H}, \mathrm{PhCHOH})$.

${ }^{13} \mathrm{C}$-NMR (100 MHz, $\left.\mathrm{CDCl}_{3}\right): \delta(\mathrm{ppm}): 145.2(\mathrm{ArC}) ; 143.5(\mathrm{ArC}) ; 138.9(\mathrm{ArC}) ; 128.6(\mathrm{ArCH}) ;$ 128.2 (ArCH); $127.8(\mathrm{ArCH}) ; 127.7(\mathrm{ArCH}) ; 127.6(\mathrm{ArCH}) ; 127.5(\mathrm{ArCH}) ; 127.1(\mathrm{ArCH}) ;$ $126.9(\mathrm{ArCH}) ; 126.3(\mathrm{ArCH}) ; 80.9(\mathrm{CH}) ; 78.1(\mathrm{C})$.

MS (ESI): $m / z(\%): 273.12$ (100), $308.16(38)\left[M+\mathrm{NH}_{4}\right]^{+}, 195.08$ (14).

HRMS (ESI) $\mathrm{m} / z$ : $\left[M+\mathrm{NH}_{4}\right]^{+}$Calcd for $\mathrm{C}_{20} \mathrm{H}_{22} \mathrm{NO}_{2}: 308.1651$; found: 308.1654 .

(S)-(-)-2-Hydroxy-1,2,2-triphenylethylacetate [(S)-HYTRA] (2)<smiles>CC(=O)OC(c1ccccc1)C(O)(c1ccccc1)c1ccccc1</smiles>

2

$\mathrm{Sc}(\mathrm{OTf})_{3}(817 \mathrm{mg}, 1.66 \mathrm{mmol}, 0.02 \mathrm{eq})$ in $\mathrm{CH}_{3} \mathrm{CN}(80 \mathrm{~mL})$ was added dropwise at room temperature to a solution of diol $\mathbf{S 2}(24.1 \mathrm{~g}, 83 \mathrm{mmol}, 1 \mathrm{eq})$ and acetic anhydride $(11.77 \mathrm{~mL}$, $124.5 \mathrm{mmol}, 1.5 \mathrm{eq}$ ) in $\mathrm{CH}_{3} \mathrm{CN}(340 \mathrm{~mL})$. The mixture was stirred for $3 \mathrm{~h}$ at room temperature and then the solid was filtered, washed with in $\mathrm{CH}_{3} \mathrm{CN}(2 \times 20)$ and dried under vacuum to furnish (S)-HYTRA 2 (23.2 g, $69.8 \mathrm{mmol}, 84 \%)$ as a white solid.

General Data: $\mathrm{C}_{22} \mathrm{H}_{20} \mathrm{O}_{3}$; FW: 332.14; TLC: $\mathrm{R}_{\mathrm{f}}=0.50$ (pentane/Et2 $\mathrm{O}$ 2:1); UV (+); mp: 249$251^{\circ} \mathrm{C} ;[\alpha]_{D}^{20}=-215.5(c=1.0$, Pyridine $)$. 
${ }^{1} \mathrm{H}-\mathrm{NMR}\left(600 \mathrm{MHz}, \mathrm{CDCl}_{3}\right): \delta(\mathrm{ppm}): 7.57-7.53(\mathrm{~m}, 2 \mathrm{H}, \mathrm{Ar}-H)$; 7.39-7.33 (m, 2H, Ar- $H$ ); 7.30$7.26(\mathrm{~m}, 1 \mathrm{H}, \mathrm{Ar}-H)$; 7.19-7.03 (m, 10H, Ar-H); 6.68 (s, 1H, PhCH); $2.82\left(\mathrm{~s}, 1 \mathrm{H}, \mathrm{Ph}_{2} \mathrm{COH}\right)$; $1.98\left(\mathrm{~s}, 3 \mathrm{H}, \mathrm{CH}_{3} \mathrm{CO}_{2} \mathrm{CH}\right)$.

${ }^{13} \mathrm{C}$-NMR $\left(151 \mathrm{MHz}, \mathrm{CDCl}_{3}\right): \delta(\mathrm{ppm}): 169.8(\mathrm{C}=\mathrm{O}) ; 144.9(\operatorname{ArC}) ; 142.8(\operatorname{ArC}) ; 135.9(\operatorname{ArC}) ;$ 128.6 (ArCH); 128.5 ( $\mathrm{ArCH}) ; 128.0(\mathrm{ArCH}) ; 127.9(\mathrm{ArCH}) ; 127.6(\mathrm{ArCH}) ; 127.5(\mathrm{ArCH}) ;$ 127.1 ( $\mathrm{ArCH}) ; 126.4(\mathrm{ArCH}) ; 126.3(\mathrm{ArCH}) ; 80.4(\mathrm{C}) ; 78.6(\mathrm{CH}) ; 21.3\left(\mathrm{CH}_{3}\right)$.

MS (ESI): $\mathrm{m} / z(\%): 274.27$ (100), 280.26 (42), $355.13(38)[\mathrm{M}+\mathrm{Na}]^{+}$.

HRMS (ESI) $\mathrm{m} / z$ : $[\mathrm{M}+\mathrm{Na}]^{+}$Calcd for $\mathrm{C}_{22} \mathrm{H}_{20} \mathrm{O}_{3} \mathrm{Na}: 355.1310$; found: 355.1359 .

\section{Synthesis of (E)-2,2-dimethyl-3-pentenal (3)}

The preparation of $(E)-2,2-$ dimethyl-3-pentenal $\mathbf{3}$ follows the procedure of a key intermediate of the Epothilone A synthesis ${ }^{[2]}$ developed by our group, with the appropriate modifications in order to get the desired product.

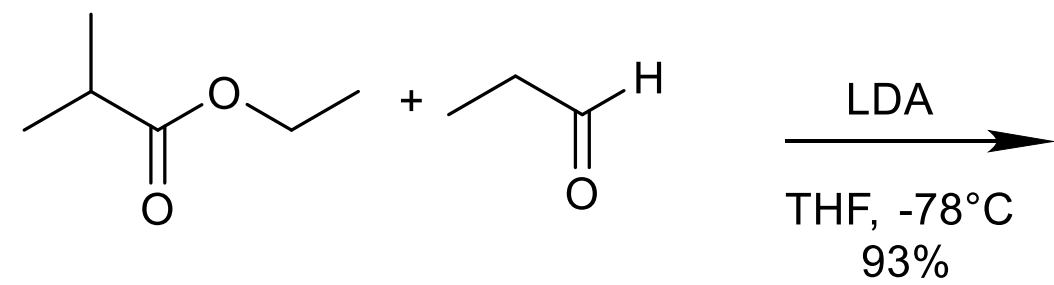<smiles>CCOC(=O)C(C)(C)C(O)CC</smiles>

S3
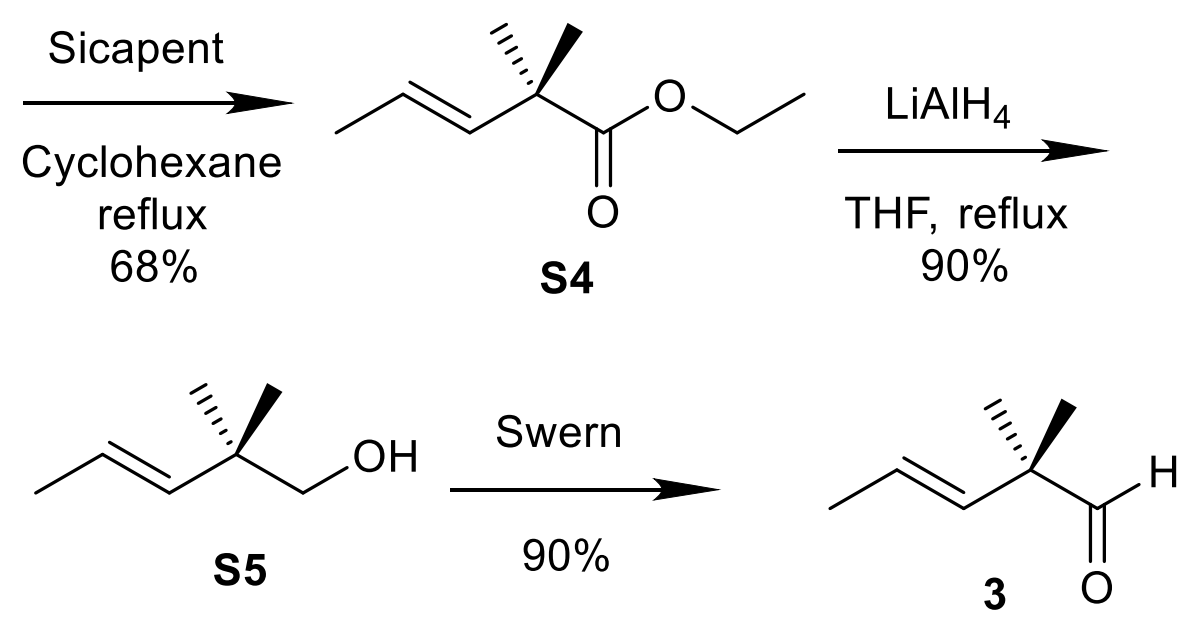

Ethyl 3-hydroxy-2,2-dimethylpentanoate (S3)<smiles>CCOC(=O)C(C)(C)C(O)CC</smiles>

S3

$n$-BuLi (133 mL, $332.38 \mathrm{mmol}, 1.1 \mathrm{eq}, 2.5 \mathrm{M}$ solution in hexane) was added dropwise at $78^{\circ} \mathrm{C}$ to a solution of diisopropylamine $(46.5 \mathrm{~mL}, 332.37 \mathrm{mmol}, 1.1 \mathrm{eq})$ in THF (300 mL). This LDA solution was stirred for $30 \mathrm{~min}$ at $0^{\circ} \mathrm{C}$ and then cooled to $-78^{\circ} \mathrm{C}$. Ethyl isobutyrate (40.6 mL, $302.16 \mathrm{mmol}, 1 \mathrm{eq})$ dissolved in THF $(58 \mathrm{~mL})$ was added dropwise and the mixture was stirred for $1 \mathrm{~h}$ at $-78^{\circ} \mathrm{C}$. Propionaldehyde $(23.8 \mathrm{~mL}, 332.38 \mathrm{mmol}, 1.1 \mathrm{eq})$ was added dropwise at $-78^{\circ} \mathrm{C}$ and then the bath was removed and the mixture was stirred for $30 \mathrm{~min}$ 
between $-50^{\circ} \mathrm{C}$ and $-10^{\circ} \mathrm{C}$. The reaction was quenched by dropwise addition of saturated aqueous $\mathrm{NH}_{4} \mathrm{Cl}$ solution (300 mL) and the organic layer was separated and the aqueous phase was extracted with $\mathrm{Et}_{2} \mathrm{O}(3 \times 200 \mathrm{~mL})$. The combined organic extracts were dried over $\mathrm{Na}_{2} \mathrm{SO}_{4}$, filtered and concentrated in vacuo. Purification of the residue by vacuum distillation through a short Vigreux column afforded $\beta$-hydroxy ester S3 (48.97 g, 281.25 mmol, 93\%) as a colorless liquid.

General Data: $\mathrm{C}_{9} \mathrm{H}_{18} \mathrm{O}_{3}$; FW: 174.13; TLC: $\mathrm{R}_{\mathrm{f}}=0.35$ (pentane/Et $2 \mathrm{O} 2: 1$ ); UV (-); Vanillin: light blue; bp: $105^{\circ} \mathrm{C}$ (5 mbar).

1H-NMR (600 MHz, CDCl 3 ): $\delta(p p m): 4.14\left(q d, J=14.2,7.2,1.1 \mathrm{~Hz}, 2 \mathrm{H}, \mathrm{OCH}_{2} \mathrm{CH}_{3}\right) ; 3.49$ (dd, $J=10.6,2.1 \mathrm{~Hz}, 1 \mathrm{H}, \mathrm{CHOH}) ; 2.35(\mathrm{~s}, 1 \mathrm{H}, \mathrm{CHOH}) ; 1.54-1.46\left(\mathrm{~m}, 1 \mathrm{H}, 1 \mathrm{CH}_{2} \mathrm{CH}_{3}\right) ; 1.29-$ $1.20\left(\mathrm{~m}, 1 \mathrm{H}, 1 \mathrm{CH}_{2} \mathrm{CH}_{3}\right) ; 1.25\left(\mathrm{t}, J=7.2 \mathrm{~Hz}, 3 \mathrm{H}, \mathrm{OCH}_{2} \mathrm{CH}_{3}\right) ; 1.17\left(\mathrm{~s}, 3 \mathrm{H}, \mathrm{CH}_{3}\right) ; 1.15(\mathrm{~s}, 3 \mathrm{H}$, $\left.\mathrm{CH}_{3}\right) ; 1.01$ (t, $\left.\mathrm{J}=7.5 \mathrm{~Hz}, 3 \mathrm{H}, \mathrm{CH}_{2} \mathrm{CH}_{3}\right)$.

${ }^{13} \mathrm{C}-N M R\left(151 \mathrm{MHz}, \mathrm{CDCl}_{3}\right): \delta(\mathrm{ppm}): 177.9(\mathrm{C}=\mathrm{O}) ; 78.5(\mathrm{CH}) ; 60.8\left(\mathrm{CH}_{2}\right) ; 47.1(\mathrm{C}) ; 24.7$ $\left(\mathrm{CH}_{2}\right) ; 22.5\left(\mathrm{CH}_{3}\right) ; 20.5\left(\mathrm{CH}_{3}\right) ; 14.3\left(\mathrm{CH}_{3}\right) ; 11.4\left(\mathrm{CH}_{3}\right)$.

IR(neat): 3464 (br); 2977 (m); 2938 (m); 2978 (m); 1712 (s); 1466 (m); 1366 (m); 1264 (s); 1139 (s); 1094 (s); $1024(\mathrm{~m}) ; 975(\mathrm{~s}) ; 861(\mathrm{~m}) \mathrm{cm}^{-1}$.

MS (ESI): $m / z(\%): 175.13(100)[M+]^{+}, 197.11(72)[M+N a]^{+}, 173.06(68), 170.05(26)$, 169.04 (22).

HRMS (ESI) $m / z:[M+H]+$ Calcd for $\mathrm{C}_{9} \mathrm{H}_{19} \mathrm{O}_{3}:$ : 175.1329; found: 175.1329 .

\section{Ethyl (E)-2,2-dimethyl-3-pentenoate (S4)}

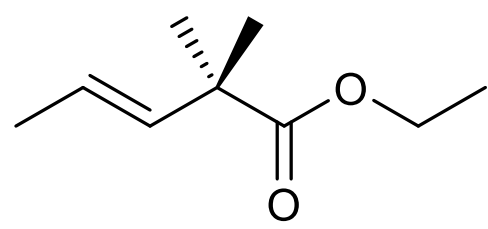

S4

Hydroxy ester S3 (48.97 g, $281.25 \mathrm{mmol}$ ) was refluxed with Sicapent ${ }^{\circledR}(70 \mathrm{~g})$ in Cyclohexane $(250 \mathrm{~mL})$ for $30 \mathrm{~min}$. The solvent was removed by atmospheric distillation and the residue was distilled under vacuum to afford ester $\mathbf{S 4}(29.85 \mathrm{~g}, 191.25 \mathrm{mmol}, 68 \%)$ as a colorless liquid.

General Data: $\mathrm{C}_{9} \mathrm{H}_{16} \mathrm{O}_{2}$; FW: 156.11 ; TLC: $\mathrm{R}_{\mathrm{f}}=0.75$ (pentane/Et $2 \mathrm{O} 2: 1$ ); UV (-); bp: 55-60 $\mathrm{C}$ (10 mbar).

${ }^{1} \mathrm{H}-N M R\left(600 \mathrm{MHz}, \mathrm{CDCl}_{3}\right): \delta(\mathrm{ppm}): 5.61(\mathrm{dq}, J=15.6,1.5 \mathrm{~Hz}, 1 \mathrm{H}, \mathrm{CH}=\mathrm{CH}) ; 5.53-5.45$ (m, $1 \mathrm{H}, \mathrm{CH}=\mathrm{CH}$ ); 4.10 (q, J = 7.3 Hz, 2H, OCH $\left.\mathrm{CH}_{3}\right) ; 1.67$ (dd, J = 6.3, $1.4 \mathrm{~Hz}, 3 \mathrm{H}, \mathrm{OCH}_{2} \mathrm{CH}_{3}$ ), $1.25\left(\mathrm{~s}, 6 \mathrm{H}, 2 \mathrm{CH}_{3}\right) ; 1.23\left(\mathrm{t}, \mathrm{J}=7.1 \mathrm{~Hz}, 3 \mathrm{H}, \mathrm{CHCH}_{3}\right)$.

${ }^{13} \mathrm{C}-N M R\left(151 \mathrm{MHz}, \mathrm{CDCl}_{3}\right): \delta(\mathrm{ppm}): 176.9(\mathrm{C}=\mathrm{O}) ; 135.7(\mathrm{CH}) ; 123.4(\mathrm{CH}) ; 60.7\left(\mathrm{CH}_{2}\right)$; $44.1(\mathrm{C}) ; 25.2\left(\mathrm{CH}_{3}\right) ; 18.2\left(\mathrm{CH}_{3}\right) ; 14.3\left(\mathrm{CH}_{3}\right)$.

IR(neat): 2980 (s); 2921 (m); $2329(\mathrm{w}) ; 1632$ (w); $1383(\mathrm{~m}) ; 1252(\mathrm{~m}) ; 1152(\mathrm{~m}) ; 1073(\mathrm{~m})$; $954 ; 814(\mathrm{w}) ; 606(\mathrm{w}) \mathrm{cm}^{-1}$.

MS (El, $70 \mathrm{eV}): m / z(\%): 156.07$ (100) [M] ${ }^{+}, 141.05$ (77), 116.03 (54), $110.02(21)$.

HRMS (El, $70 \mathrm{eV}) \mathrm{m} / \mathrm{z}$ : [M] ${ }^{+}$Calcd for $\mathrm{C}_{9} \mathrm{H}_{16} \mathrm{O}_{2}$ : 156.1145; found: 156.1145 .

(E)- 2,2-dimethyl-3-penten-1-ol (S5)

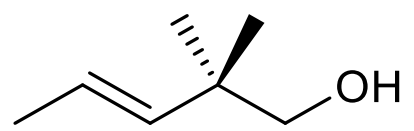


To a solution of ester $\mathbf{S 4}(16.37 \mathrm{~g}, 104.94 \mathrm{mmol}, 1 \mathrm{eq})$ in THF (150 mL), $\mathrm{LiAlH}_{4}(7.98 \mathrm{~g}$, $209.87 \mathrm{mmol}, 2 \mathrm{eq})$ was added and the mixture was refluxed for $2 \mathrm{~h}$. After cooling to $0^{\circ} \mathrm{C}$, $\mathrm{Et}{ }_{2} \mathrm{O}(100 \mathrm{~mL})$ was added and the reaction was quenched by dropwise addition of water (10 $\mathrm{mL}$ ). The mixture was stirred for $30 \mathrm{~min}$ at room temperature until a white precipitate was formed, which was filtered off by suction through Celite and washed with Et2O (3x200 mL). The filtrate and the washings were combined and concentrated in vacuo to furnish crude alcohol 6 (10.77 g, $94.45 \mathrm{mmol}, 90 \%)$ as a colorless liquid, which was used for the preparation of aldehyde $\mathbf{S 5}$ without further purification.

General Data: $\mathrm{C}_{7} \mathrm{H}_{14} \mathrm{O}$; FW: 114.10; TLC: $\mathrm{R}_{\mathrm{f}}=0.35$ (pentane/Et ${ }_{2} \mathrm{O}$ 2:1); UV (-); dark blue; bp: $152-160^{\circ} \mathrm{C}$.

${ }^{1} \mathrm{H}-\mathrm{NMR}\left(400 \mathrm{MHz}, \mathrm{CDCl}_{3}\right)$ : $\delta$ (ppm): 5.50-5.43 (m, $\left.1 \mathrm{H}, \mathrm{CH}=\mathrm{CH}\right) ; 5.35$ (dd, $J=15.6,1.3 \mathrm{~Hz}$, $1 \mathrm{H}, \mathrm{CH}=\mathrm{CH}) ; 3.27(\mathrm{~s}, 2 \mathrm{H}, \mathrm{CH} 2 \mathrm{OH}) ; 1.69$ (dd, $\left.J=6.5,1.2 \mathrm{~Hz}, 3 \mathrm{H}, \mathrm{CHCH}_{3}\right) ; 0.977(\mathrm{~s}, 6 \mathrm{H}$, $2 \mathrm{CH} \mathrm{H}_{3}$.

${ }^{13} \mathrm{C}-N M R\left(100 \mathrm{MHz}, \mathrm{CDCl}_{3}\right): \delta(\mathrm{ppm}): 137.9(\mathrm{CH}) ; 124.3(\mathrm{CH}) ; 71.7\left(\mathrm{CH}_{2}\right) ; 38.5(\mathrm{C}) ; 24.1$ $\left(\mathrm{CH}_{3}\right) ; 18.4\left(\mathrm{CH}_{3}\right)$.

IR(neat): 3366 (br); 3027 (w); 2960 (m); 2930 (m); 2869 (m); 1723 (w); 1450 (m); 1378 (m); $1248(\mathrm{~m}) ; 1158(\mathrm{w}) ; 1044(\mathrm{~m}) ; 970(\mathrm{~m}) ; 894(\mathrm{w}) ; 697(\mathrm{w}) ; 610(\mathrm{w}) \mathrm{cm}^{-1}$.

MS (ESI): $m / z(\%): 175.13(100), 115.11(58)[M+H]^{+}, 141.05(77), 116.03(54), 110.02(21)$. HRMS (ESI) $m / z$ : $[M+H]^{+}$Calcd for $\mathrm{C}_{7} \mathrm{H}_{15} \mathrm{O}$ : 115.1123 ; found: 115.1119 .

(E)-2,2-dimethyl-3-pentenal (3)

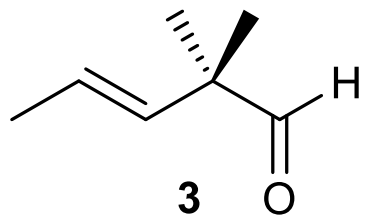

DMSO (11.2 mL, $157.4 \mathrm{mmol}, 2.0 \mathrm{eq})$ in $\mathrm{CH}_{2} \mathrm{Cl}_{2}(45 \mathrm{~mL})$ was added dropwise at $-78^{\circ} \mathrm{C}$ to a stirred solution of $(\mathrm{COCl})_{2}(8.1 \mathrm{~mL}, 94.44 \mathrm{mmol}, 1.2 \mathrm{eq})$ in $\mathrm{CH}_{2} \mathrm{Cl}_{2}(210 \mathrm{~mL})$. The mixture was stirred for $10 \mathrm{~min}$ at $-78^{\circ} \mathrm{C}$. The crude (E)-2,2-dimethyl-3-penten-1-ol S5 (8.97 g, 78.7 mmol, $1 \mathrm{eq})$ dissolved in $\mathrm{CH}_{2} \mathrm{Cl}_{2}(60 \mathrm{~mL})$ was added dropwise at $-78^{\circ} \mathrm{C}$ and the mixture was stirred for $1 \mathrm{~h}$ at $-78^{\circ} \mathrm{C}$. The reaction was quenched by dropwise addition of NEt3 $(54.6 \mathrm{~mL}$, $393.5 \mathrm{mmol}, 5.0 \mathrm{eq}$ ) and the mixture was warmed to room temperature over $45 \mathrm{~min}$. Water was added $(250 \mathrm{~mL})$ and the mixture was stirred for $10 \mathrm{~min}$. The organic layer was separated and the aqueous phase was extracted with $\mathrm{CH}_{2} \mathrm{Cl}_{2}(3 \times 150 \mathrm{~mL})$. The combined organic extracts were washed with $1 \mathrm{M} \mathrm{HCl}(150 \mathrm{~mL})$, dried over $\mathrm{Na}_{2} \mathrm{SO}_{4}$, filtered and concentrated in vacuo. Vacuum distillation of the residue afforded aldehyde 3 (7.9 g, $70.83 \mathrm{mmol}, 90 \%)$ as a colorless liquid, which was stored in the freezer.

General Data: $\mathrm{C}_{7} \mathrm{H}_{12} \mathrm{O}$; FW: 112.09; TLC: $\mathrm{R}_{\mathrm{f}}=0.80$ (pentane/Et $\mathrm{F}_{2} \mathrm{O}$ 5:1); UV (-); bp: 127$128^{\circ} \mathrm{C}$.

${ }^{1} \mathrm{H}-N M R\left(600 \mathrm{MHz}, \mathrm{CDCl}_{3}\right): \delta$ (ppm): 9.32 (s, $\left.1 \mathrm{H}, \mathrm{CHO}\right) ; 5.57-5.49(\mathrm{~m}, 1 \mathrm{H}, \mathrm{CH}=\mathrm{CH}) ; 5.36$ $(\mathrm{dd}, J=15.7,1.6 \mathrm{~Hz}, 1 \mathrm{H}, \mathrm{CH}=\mathrm{CH}) ; 1.71$ (dd, $\left.J=6.4,1.5 \mathrm{~Hz}, 3 \mathrm{H}, \mathrm{CHCH}_{3}\right) ; 1.14(\mathrm{~s}, 6 \mathrm{H}$, $2 \mathrm{CH}_{3}$.

${ }^{13} \mathrm{C}-N M R\left(151 \mathrm{MHz}, \mathrm{CDCl}_{3}\right): \delta(\mathrm{ppm}): 203.0(\mathrm{C}=\mathrm{O}) ; 132.6(\mathrm{CH}) ; 127.2(\mathrm{CH}) ; 48.6(\mathrm{C}) ; 21.6$ $\left(\mathrm{CH}_{3}\right) ; 18.5\left(\mathrm{CH}_{3}\right)$.

IR(CDCl $)$ : 3014 (m); 2935 (m); 2349 (w); 2328 (w); 2256 (w); 1723 (s); 1651 (w); 1466 (w); 1309 (w); $1213(\mathrm{w}) ; 1035(\mathrm{w}) ; 906(\mathrm{~s}) ; 728(\mathrm{~s}) ; 649(\mathrm{~m}) \mathrm{cm}^{-1}$.

MS (ESI): $m / z(\%): 109.10$ (100), 111.08 (35), 112.11 (30), 195.08 (31), 107.08 (21), 113.09 (4) $[\mathrm{M}+\mathrm{H}]^{+}$.

HRMS (ESI) $m / z:[M+H]^{+}$Calcd for $\mathrm{C}_{7} \mathrm{H}_{13} \mathrm{O}$ : 113.0966; found: 113.0976 . 
(1S)-2-Hydoxy-1,2,2-triphenylethl (3S,5E)-3hydoxy-4,4-dimethyl-5-heptenoate (4)

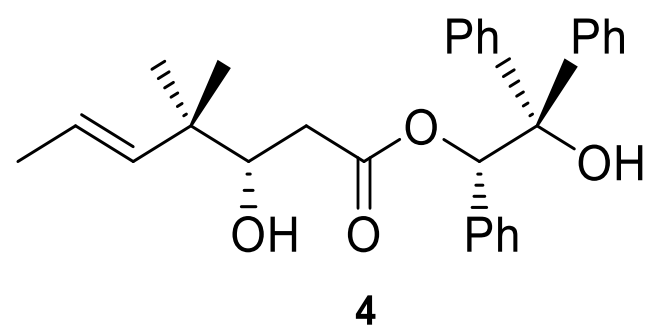

n-BuLi (22.5 mL, $56.32 \mathrm{mmol}, 2.2 \mathrm{eq}, 2.5 \mathrm{M}$ solution in hexane) was added dropwise at $78^{\circ} \mathrm{C}$ to a solution of diisopropylamine $(7.9 \mathrm{~mL}, 56.32 \mathrm{mmol}, 2.2 \mathrm{eq})$ in THF $(80 \mathrm{~mL})$. This LDA solution was stirred for $30 \mathrm{~min}$ at $0^{\circ} \mathrm{C}$ and then added dropwise to a solution of (S)HYTRA $2(8.5 \mathrm{~g}, 25.6 \mathrm{mmol}, 1 \mathrm{eq})$ in THF $(150 \mathrm{~mL})$ at $-78^{\circ} \mathrm{C}$. The mixture was stirred for 1 $\mathrm{h}$ at $0^{\circ} \mathrm{C}$. The resulting yellow-orange solution was cooled to $-78^{\circ} \mathrm{C}$ and a solution of aldehyde $3(3.44 \mathrm{~g}, 30.7 \mathrm{mmol}, 1.2 \mathrm{eq})$ in THF $(7 \mathrm{~mL})$ was added dropwise. The mixture was stirred for $2 \mathrm{~h} 30 \mathrm{~min}$ at $-78^{\circ} \mathrm{C}$. The reaction was quenched by dropwise addition of saturated aqueous $\mathrm{NH}_{4} \mathrm{Cl}$ solution $(150 \mathrm{~mL}$ ) and the mixture was allowed to warm to room temperature over $30 \mathrm{~min}$. The organic layer was separated and the aqueous phase was extracted with $\mathrm{CH}_{2} \mathrm{Cl}_{2}(3 \times 100 \mathrm{~mL})$. The combined organic extracts were dried over $\mathrm{Na}_{2} \mathrm{SO}_{4}$, filtered and concentrated in vacuo. Purification of the residue by flash chromatography (pentane/Et $2 \mathrm{O} 3: 1)$ afforded $\beta$-hydroxy ester 4 (8.75 g, $19.71 \mathrm{mmol}, 77 \%, 96 \%$ de) as a colorless solid.

General Data: $\mathrm{C}_{29} \mathrm{H}_{32} \mathrm{O}_{4}$; FW: 444.23; TLC: $\mathrm{R}_{\mathrm{f}}=0.45$ (pentane/Et $2 \mathrm{O} 2: 1$ ); UV (+); Vanillin: green; $\mathrm{mp}: 120-126^{\circ} \mathrm{C} ;[\alpha]_{D}^{20}=-167.8\left(c=1.0, \mathrm{CHCl}_{3}\right)$.

${ }^{1} \mathrm{H}-\mathrm{NMR}\left(400 \mathrm{MHz}, \mathrm{CDCl}_{3}\right): \delta$ (ppm): 7.61-7.53 (m, 2H, Ar-H); 7.41-7.33 (m, 2H, Ar-H); 7.31$7.26(\mathrm{~m}, 1 \mathrm{H}, \mathrm{Ar}-H)$; 7.22-7.09 (m, 8H, Ar-H); 7.08-7.05 (m, 2H, Ar-H); 6.71 (s, 1H, CHPh); 5.45-5.34 (m, $1 \mathrm{H}, \mathrm{CH}=\mathrm{CH}) ; 5.31(\mathrm{dd}, J=15.9,1.1 \mathrm{~Hz}, 1 \mathrm{H}, \mathrm{CH}=\mathrm{CH}) ; 3.51$ (dd, J= 10.4, 2.2 $\mathrm{Hz}, 1 \mathrm{H}, \mathrm{CHOH}) ; 2.86\left(\mathrm{~s}, 1 \mathrm{H}, \mathrm{CHOH}\right.$; 2.36 (dd, J=15.8, $2.2 \mathrm{~Hz}, 1 \mathrm{H}, 1 \mathrm{COCH}_{2}$ ); 2.24 (dd, J $\left.=15.8,10.3 \mathrm{~Hz}, 1 \mathrm{H}, 1 \mathrm{COCH}_{2}\right) ; 1.65\left(\mathrm{dd}, J=5.8,1.0 \mathrm{~Hz}, 3 \mathrm{H}, \mathrm{CHCH}_{3}\right) ; 0.923\left(\mathrm{~s}, 3 \mathrm{H}, 1 \mathrm{CCH}_{3}\right)$; $0.916\left(\mathrm{~s}, 3 \mathrm{H}, 1 \mathrm{CCH}_{3}\right)$.

${ }^{13} \mathrm{C}-N M R\left(100 \mathrm{MHz}, \mathrm{CDCl}_{3}\right): \delta$ (ppm): $172.4(\mathrm{C}=\mathrm{O}) ; 144.8(\mathrm{ArC}) ; 142.7(\mathrm{ArC}) ; 137.1(\mathrm{CH})$; 135.6 (ArC); $128.5(\mathrm{ArCH}) ; 128.5(\mathrm{ArCH}) ; 128.1(\mathrm{ArCH}) ; 127.9(\mathrm{ArCH}) ; 127.7(\mathrm{ArCH}) ; 127.5$ $(\mathrm{ArCH}) ; 127.2(\mathrm{ArCH}) ; 126.4(\mathrm{ArCH}) ; 126.3(\mathrm{ArCH}) ; 123.9(\mathrm{CH}) ; 80.5(\mathrm{CHPh}) ; 79.1$ $(\mathrm{CPh} 2 \mathrm{OH}) ; 74.9(\mathrm{CHOH}) ; 40.2(\mathrm{C}) ; 37.5\left(\mathrm{CH}_{2}\right) ; 23.8\left(\mathrm{CH}_{3}\right) ; 22.9\left(\mathrm{CH}_{3}\right) ; 18.4\left(\mathrm{CH}_{3}\right)$.

IR(neat): 3536 (br); 3029 (w); 2965 (w); 2876 (w); 1718 (m); 1600 (w); 1493 (m); 1447 (m); $1323(\mathrm{~m}) ; 1247(\mathrm{~m}) ; 1150(\mathrm{~m}) ; 1033(\mathrm{~m}) ; 974(\mathrm{~m}) ; 891(\mathrm{~m}) ; 752(\mathrm{~m}) ; 695(\mathrm{~s}) ; 612(\mathrm{~m}) \mathrm{cm}^{-1}$. MS (ESI): $m / z(\%): 273.13(100), 467.22(97)[M+\mathrm{Na}]^{+}, 911.45(40)[2 \mathrm{M}+\mathrm{Na}]^{+}, 195.08(31)$, $255.12(18), 444.23(<0.4)[M]^{+}$.

HRMS (ESI) $\mathrm{m} / \mathrm{z}$ : $[M+\mathrm{Na}]^{+}$Calcd for $\mathrm{C}_{29} \mathrm{H}_{32} \mathrm{O}_{4} \mathrm{Na}$ : 467.2098; found: 467.2198.

(3S,5E)-4,4-dimethyl-5-heptene-1,3-diol (5)

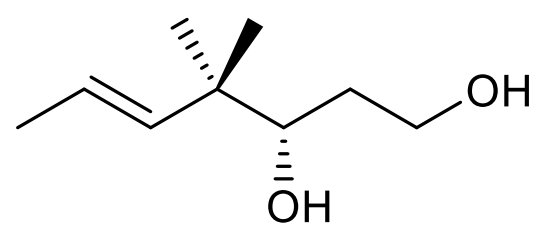


$\mathrm{LiAlH}_{4}(5.2 \mathrm{~g}, 137.97 \mathrm{mmol}, 7.0 \mathrm{eq})$ was added portionwise to a refluxing solution of $\beta$ hydroxy ester $4(8.75 \mathrm{~g}, 19.71 \mathrm{mmol}, 1 \mathrm{eq})$ in $\mathrm{Et}_{2} \mathrm{O}(210 \mathrm{~mL})$, during a period of $2 \mathrm{~h}$. Reflux was continued for $30 \mathrm{~min}$. After cooling to $0^{\circ} \mathrm{C}$, the reaction was quenched by dropwise addition of water $(10 \mathrm{~mL})$. Then $\mathrm{Et}_{2} \mathrm{O}(150 \mathrm{~mL})$ was added and the mixture was stirred for $30 \mathrm{~min}$ at room temperature until a white precipitate was formed. The precipitate was filtered off by suction through Celite and washed with $\mathrm{Et}_{2} \mathrm{O}(4 \times 100 \mathrm{~mL})$. The filtrate and the washings were combined and concentrated in vacuo. Purification of the residue by flash chromatography (pentane/Et ${ }_{2} \mathrm{O} 2: 1$ then pure $\left.\mathrm{Et}_{2} \mathrm{O}\right)$ afforded diol $5(2.8 \mathrm{~g}, 17.74 \mathrm{mmol}, 90 \%)$ as a colorless oil and (S)-(-)-1,1,2-triphenyl-1,2-ethandiol S2 (5.1 g, $17.74 \mathrm{mmol}, 90 \%)$.

General Data: $\mathrm{C}_{9} \mathrm{H}_{18} \mathrm{O}_{2}$; FW: 158.13; TLC: $\mathrm{R}_{\mathrm{f}}=0.30\left(\mathrm{Et}_{2} \mathrm{O}\right)$; UV (-); Vanillin: dark blue; $[\alpha]_{D}^{20}$ $=-7.28\left(c=0.7, \mathrm{CHCl}_{3}\right)$.

1H-NMR (400 MHz, $\left.\mathrm{CDCl}_{3}\right): \delta(\mathrm{ppm}): 5.52-5.43(\mathrm{~m}, 1 \mathrm{H}, \mathrm{CH}=\mathrm{CH}) ; 5.38(\mathrm{dq}, \mathrm{J}=15.6,1.3 \mathrm{~Hz}$, $1 \mathrm{H}, \mathrm{CH}=\mathrm{CH}) ; 3.86-3.76\left(\mathrm{~m}, 2 \mathrm{H}, \mathrm{CH}_{2} \mathrm{OH}\right) ; 3.47$ (dd, J = 10.7, $\left.2.2 \mathrm{~Hz}, 1 \mathrm{H}, \mathrm{CHOH}\right) ; 2.42$ (s, $2 \mathrm{H}, 2 \mathrm{OH}) ; 1.74-1.66\left(\mathrm{~m}, 1 \mathrm{H}, 1 \mathrm{CH}_{2} \mathrm{CH}_{2} \mathrm{OH}\right) ; 1.69$ (dd, $\left.J=6.0,1.3 \mathrm{~Hz}, 3 \mathrm{H}, \mathrm{CHCH}_{3}\right) ; 1.62-$ $1.52\left(\mathrm{~m}, 1 \mathrm{H}, 1 \mathrm{CH}_{2} \mathrm{CH}_{2} \mathrm{OH}\right) ; 0.980\left(\mathrm{~s}, 6 \mathrm{H}, 2 \mathrm{CCH}_{3}\right)$.

${ }^{13}$ C-NMR (100 MHz, CDCl $)$ : $\delta(p p m): 137.8(\mathrm{CH}) ; 124.6(\mathrm{CH}) ; 79.0(\mathrm{CH}) ; 62.7\left(\mathrm{CH}_{2}\right) ; 40.8$ $(\mathrm{C}) ; 32.8\left(\mathrm{CH}_{2}\right) ; 23.8\left(\mathrm{CH}_{3}\right) ; 22.3\left(\mathrm{CH}_{3}\right) ; 18.4\left(\mathrm{CH}_{3}\right)$.

IR(neat): 3336 (br); 3025 (w); 2961 (m); 2932 (m); 2876 (m); 1668 (w); 1468 (m); 1447 (m); $1382(\mathrm{~m}) ; 1320(\mathrm{~m}) ; 1192(\mathrm{w}) ; 1049(\mathrm{~s}) ; 971(\mathrm{~s}) ; 878(\mathrm{~m}) ; 832(\mathrm{w}) \mathrm{cm}^{-1}$.

MS (ESI): $m / z(\%): 181.1207(100)[M+N a]^{+}, 123.12(42), 158.13(<0.4)\left[M^{+}\right.$.

HRMS (ESI) $\mathrm{m} / \mathrm{z}$ : $[M+\mathrm{Na}]^{+}$Calcd for $\mathrm{C}_{9} \mathrm{H}_{18} \mathrm{O}_{2} \mathrm{Na}: 181.1205$; found: 181.1207.

(S,E)-3,3,9,9-tetraethyl-5-(2-methylpent-3-en-2-yl)-4,8-dioxa-3,9-disilaundecane (6)

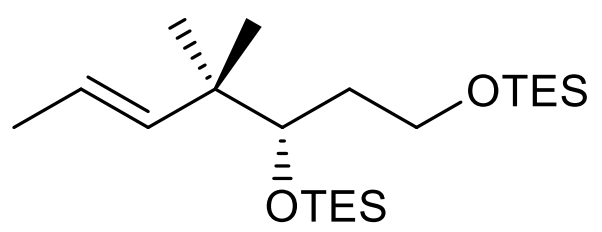

6

To a solution of diol 5 (1.96 g, $12.4 \mathrm{mmol}, 1 \mathrm{eq})$ in $\mathrm{CH}_{2} \mathrm{Cl}_{2}(120 \mathrm{~mL}), 2,6$-Lutidine (4.3 mL, $37.2 \mathrm{mmol}, 3 \mathrm{eq})$ and TESOTf $(5.9 \mathrm{~mL}, 26.0 \mathrm{mmol}, 2.1 \mathrm{eq})$ were sequentially added dropwise at $-78^{\circ} \mathrm{C}$. The mixture was stirred for $30 \mathrm{~min}$ at $-78^{\circ} \mathrm{C}$ and for $1 \mathrm{~h}$ at $0^{\circ} \mathrm{C}$. Saturated aqueous $\mathrm{NaHCO}_{3}$ solution $(100 \mathrm{~mL})$ was added and the layers were separated. The aqueous phase was extracted with $\mathrm{CH}_{2} \mathrm{Cl}_{2}(3 \times 80 \mathrm{~mL})$ and the combined organic extracts were dried over $\mathrm{Na}_{2} \mathrm{SO}_{4}$, filtered and concentrated in vacuo. Purification of the residue by flash chromatography (pentane) furnished compound $6(4.74 \mathrm{~g}, 12.28 \mathrm{mmol}, 99 \%)$ as a colorless liquid.

General Data: $\mathrm{C}_{21} \mathrm{H}_{46} \mathrm{O}_{2} \mathrm{Si}_{2}$; FW: 386.3; TLC: $\mathrm{R}_{\mathrm{f}}=0.20$ (pentane); UV (-); Vanillin: black; $[\alpha]_{D}^{20}=-15.00\left(c=0.5, \mathrm{CHCl}_{3}\right)$.

${ }^{1} \mathrm{H}-\mathrm{NMR}\left(600 \mathrm{MHz}, \mathrm{CDCl}_{3}\right): \delta(\mathrm{ppm}): 5.43(\mathrm{dq}, J=15.6,1.2 \mathrm{~Hz}, 1 \mathrm{H}, \mathrm{CH}=\mathrm{CH}) ; 5.38-5.30(\mathrm{~m}$, $1 \mathrm{H}, \mathrm{CH}=\mathrm{CH})$; 3.71-3.62 (m, $\left.1 \mathrm{H}, 1 \mathrm{CH}_{2} \mathrm{OSi}\right) ; 3.61-3.53\left(\mathrm{~m}, 1 \mathrm{H}, 1 \mathrm{CH}_{2} \mathrm{OSi}\right) ; 3.46(\mathrm{dd}, \mathrm{J}=8.4$, $2.8 \mathrm{~Hz}, 1 \mathrm{H}, \mathrm{CHOSi}) ; 1.77-1.67\left(\mathrm{~m}, 1 \mathrm{H}, 1 \mathrm{CH}_{2} \mathrm{CH}_{2} \mathrm{O}\right) ; 1.65\left(\mathrm{dd}, J=6.2,1.3 \mathrm{~Hz}, 3 \mathrm{H}, \mathrm{CHCH}_{3}\right)$; 1.53-1.43 (m, $\left.1 \mathrm{H}, 1 \mathrm{CH}_{2} \mathrm{CH}_{2} \mathrm{O}\right) ; 1.00-0.89\left(\mathrm{~m}, 18 \mathrm{H}, 2 \mathrm{OSi}\left(\mathrm{CH}_{2} \mathrm{CH}_{3}\right)_{3}\right) ; 0.969\left(\mathrm{~s}, 3 \mathrm{H}, 1 \mathrm{CCH}_{3}\right)$; $0.940\left(\mathrm{~s}, 3 \mathrm{H}, 1 \mathrm{CCH}_{3}\right) ; 0.654-0.552\left(\mathrm{~m}, 12 \mathrm{H}, 2 \mathrm{OSi}\left(\mathrm{CH}_{2} \mathrm{CH}_{3}\right)_{3}\right)$.

${ }^{13} \mathrm{C}$-NMR (151 MHz, CDCl $)$ : $\delta(\mathrm{ppm}): 139.4(\mathrm{CH}) ; 121.6(\mathrm{CH}) ; 77.0(\mathrm{CH}) ; 61.0\left(\mathrm{CH}_{2}\right) ; 41.2$ (C); $36.8\left(\mathrm{CH}_{2}\right) ; 24.7\left(\mathrm{CH}_{3}\right) ; 23.0\left(\mathrm{CH}_{3}\right) ; 18.4\left(\mathrm{CH}_{3}\right) ; 7.3\left(\mathrm{CH}_{3}\right) ; 6.9\left(\mathrm{CH}_{3}\right) ; 5.6\left(\mathrm{CH}_{2}\right) ; 4.6$ $\left(\mathrm{CH}_{2}\right)$. 
IR(neat): 2954 (m); 2915 (m); 2876 (m); 1632 (w); 1536 (w); 1460 (m); 1379 (m); 1238 (m); 1099 (s); 1006 (s); 800 (w); 725 (s); $674(\mathrm{~m}) \mathrm{cm}^{-1}$.

MS (ESI): $m / z(\%): 506.53$ (100), $156.12(3), 449.34(2), 387.31(<0.4)[M+\mathrm{H}]^{+}$.

HRMS (ESI) $\mathrm{m} / z$ : $[M+\mathrm{H}]^{+}$Calcd for $\mathrm{C}_{21} \mathrm{H}_{47} \mathrm{O}_{2} \mathrm{Si}_{2}: 387.3115$; found: 387.3111 .

\section{(S)-2,2-dimethyl-3,5-bis((triethylsilyl)oxy)pentanal (7)}

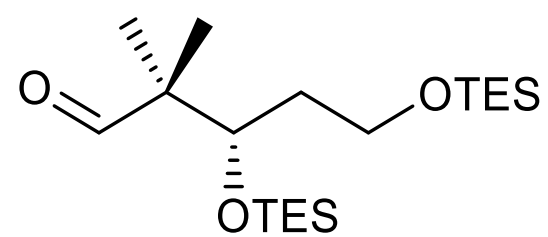

7

A stream of ozone in oxygen was bubbled through a solution of compound 6 (4.74 g, 12.28 mmol, $1 \mathrm{eq})$ in $\mathrm{CH}_{2} \mathrm{Cl}_{2}(400 \mathrm{~mL})$ at $-78^{\circ} \mathrm{C}$ until the blue color of the solution persisted. Then oxygen was bubbled for 10 min to remove excess of ozone and $\mathrm{PPh}_{3}(3.86 \mathrm{~g}, 14.73 \mathrm{mmol}$, $1.2 \mathrm{eq}$ ) was added at $-78^{\circ} \mathrm{C}$. The mixture was warmed to room temperature and stirred for $3 \mathrm{~h}$. The crude was concentrated in vacuo, then pentane $(300 \mathrm{~mL})$ was added (the triphenylphosphine oxide is insoluble in pentane) and the mixture was filtered on paper, with additional pentane washes. The filtrate was concentrated in vacuo and the residue was purified by flash chromatography (pentane/Et ${ }_{2} \mathrm{O} 100: 1$ to 50:1) to furnish aldehyde 7 (3.08 $\mathrm{g}, 8.22 \mathrm{mmol}, 67 \%$ ) as a colorless liquid.

General Data: $\mathrm{C}_{19} \mathrm{H}_{42} \mathrm{O}_{3} \mathrm{Si}_{2}$; FW: 374.27; TLC: $\mathrm{R}_{\mathrm{f}}=0.35$ (pentane/Et $2 \mathrm{O} 50: 1$ ); UV (-); Vanillin: violet; $[\alpha]_{D}^{20}=+10.11\left(c=0.9, \mathrm{CHCl}_{3}\right)$.

${ }^{1} \mathrm{H}-\mathrm{NMR}\left(400 \mathrm{MHz}, \mathrm{CDCl}_{3}\right): \delta(\mathrm{ppm}): 9.57$ (s, $\left.1 \mathrm{H}, \mathrm{CHO}\right) ; 3.99(\mathrm{dd}, J=8.2,3.0 \mathrm{~Hz}, 1 \mathrm{H}$, $\mathrm{CHOSi}) ; 3.71-3.58\left(\mathrm{~m}, 2 \mathrm{H}, \mathrm{CH}_{2} \mathrm{OSi}\right) ; 1.74-1.64\left(\mathrm{~m}, 1 \mathrm{H}, 1 \mathrm{CH}_{2} \mathrm{CH}_{2} \mathrm{O}\right) ; 1.62-1.52(\mathrm{~m}, 1 \mathrm{H}$, $\left.1 \mathrm{CH}_{2} \mathrm{CH}_{2} \mathrm{O}\right) ; 1.04\left(\mathrm{~s}, 3 \mathrm{H}, 1 \mathrm{CCH}_{3}\right) ; 1.00\left(\mathrm{~s}, 3 \mathrm{H}, 1 \mathrm{CCH}_{3}\right) ; 0.984-0.908(\mathrm{~m}, 18 \mathrm{H}$, $\left.2 \mathrm{OSi}\left(\mathrm{CH}_{2} \mathrm{CH}_{3}\right)_{3}\right) ; 0.659-0.519\left(\mathrm{~m}, 12 \mathrm{H}, 2 \mathrm{OSi}\left(\mathrm{CH}_{2} \mathrm{CH}_{3}\right)_{3}\right)$.

${ }^{13} \mathrm{C}-N M R\left(100 \mathrm{MHz}, \mathrm{CDCl}_{3}\right): \delta(\mathrm{ppm}): 206.6(\mathrm{C}=\mathrm{O}) ; 73.4(\mathrm{CH}) ; 59.8\left(\mathrm{CH}_{2}\right) ; 51.3(\mathrm{C}) ; 36.6$ $\left(\mathrm{CH}_{2}\right) ; 19.1\left(\mathrm{CH}_{3}\right) ; 17.6\left(\mathrm{CH}_{3}\right) ; 7.1\left(\mathrm{CH}_{3}\right) ; 6.9\left(\mathrm{CH}_{3}\right) ; 5.5\left(\mathrm{CH}_{2}\right) ; 4.6\left(\mathrm{CH}_{2}\right)$.

IR(neat): 2955 (s); 2932 (m); 2877 (s); 2731 (w); 1730 (m); 1671 (w); 1460 (m); 1415 (m); $1378(\mathrm{~m}) ; 1238(\mathrm{~m}) ; 1085(\mathrm{~s}) ; 1006(\mathrm{~s}) ; 962(\mathrm{~m}) ; 918(\mathrm{~m}) ; 821(\mathrm{~m}) ; 725(\mathrm{~s}) \mathrm{cm}^{-1}$.

MS (ESI): $m / z(\%): 243.17$ (100), $259.17(5), 156.12(4), 503.35(2), 375.27(<0.4)[M+\mathrm{H}]^{+}$. HRMS (ESI) $\mathrm{m} / \mathrm{z}:[\mathrm{M}+\mathrm{H}]^{+}$Calcd for $\mathrm{C}_{19} \mathrm{H}_{43} \mathrm{O}_{3} \mathrm{Si}_{2}: 375.2751$; found: 375.2743 .

(4S,6S)-5,5-dimethyl-6,8-bis((triethylsilyl)oxy)oct-1-en-4-ol (9)

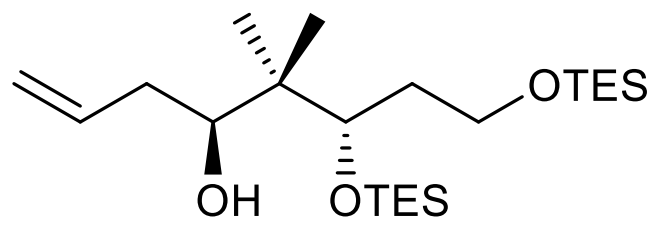

9

A solution of aldehyde $7(2.23 \mathrm{~g}, 5.96 \mathrm{mmol}, 1 \mathrm{eq})$ in $\mathrm{CH}_{2} \mathrm{Cl}_{2}(10 \mathrm{~mL})$ was added to a solution of $(R, R)-8^{[3]}(3.95 \mathrm{~g}, 7.15 \mathrm{mmol}, 1.2 \mathrm{eq})$ in $\mathrm{CH}_{2} \mathrm{Cl}_{2}(50 \mathrm{~mL})$ at $-15^{\circ} \mathrm{C}$ (ice/acetone bath). Then $\mathrm{Sc}(\mathrm{OTf})_{3}(147 \mathrm{mg}, 0.298 \mathrm{mmol}, 0.05 \mathrm{eq})$ was added, the flask was sealed under nitrogen, transferred into a freezer $\left(-15^{\circ} \mathrm{C}\right)$ and stirred for $48 \mathrm{~h}$. TBAF trihydrate $(1.88 \mathrm{~g}, 5.96 \mathrm{mmol}, 1$ eq) was added and the mixture was stirred for $30 \mathrm{~min}$ at room temperature. The solvent was evaporated and the residue was purified by flash chromatography (pentane/Et ${ }_{2} \mathrm{O} 50: 1$, then hexane/EtOAc/NEt3 1:1:0.1) to furnish the allylic alcohol $9(2.1 \mathrm{~g}, 5.06 \mathrm{mmol}, 85 \%, 84 \%$ de) 
as a colorless liquid and the recovered diamine of the Leighton reagent ${ }^{[3]}(3.64 \mathrm{~g}, 8.08 \mathrm{mmol}$, $87 \%$ ) as a yellow paste. Analysis by ${ }^{1} \mathrm{H}$ and ${ }^{13} \mathrm{C}$ NMR showed a $11: 1$ mixture of anti and syn diastereoisomers.

General Data: $\mathrm{C}_{22} \mathrm{H}_{48} \mathrm{O}_{3} \mathrm{Si}_{2} ; \mathrm{FW}: 416.31$; TLC: $\mathrm{R}_{\mathrm{f}}=0.2$ (pentane/Et $2 \mathrm{O} 50: 1$ ); UV (-); Vanillin: dark blue; $[\alpha]_{D}^{20}=-38.00\left(c=0.45, \mathrm{CHCl}_{3}\right)$.

${ }^{1} \mathrm{H}-\mathrm{NMR}\left(600 \mathrm{MHz}^{\mathrm{C}} \mathrm{CDCl}_{3}\right): \delta(\mathrm{ppm}): 5.97-5.89\left(\mathrm{~m}, 1 \mathrm{H}, \mathrm{CH}=\mathrm{CH}_{2}\right) ; 5.13-5.03(\mathrm{~m}, 2 \mathrm{H}$, $\left.\mathrm{CH}=\mathrm{CH}_{2}\right) ; 4.28(\mathrm{~s}, 1 \mathrm{H}, \mathrm{CHOH}) ; 3.78(\mathrm{dd}, \mathrm{J}=9.5,3.2 \mathrm{~Hz}, 1 \mathrm{H}, \mathrm{CHOH}) ; 3.73(\mathrm{~m}, 1 \mathrm{H}$, $1 \mathrm{CH}_{2} \mathrm{OSi}$ ); 3.69 (dd, $\left.J=8.6,2.2 \mathrm{~Hz}, 1 \mathrm{H}, \mathrm{CHOSi}\right) ; 3.63\left(\mathrm{~m}, 1 \mathrm{H}, 1 \mathrm{CH}_{2} \mathrm{OSi}\right) ; 2.16-2.06(\mathrm{~m}, 2 \mathrm{H}$, $\left.\mathrm{CH}_{2} \mathrm{CH}\right) ; 1.90-1.83\left(\mathrm{~m}, 1 \mathrm{H}, 1 \mathrm{CH}_{2} \mathrm{CH}_{2} \mathrm{O}\right) ; 1.74-1.65\left(\mathrm{~m}, 1 \mathrm{H}, 1 \mathrm{CH}_{2} \mathrm{CH}_{2} \mathrm{O}\right) ; 1.01(\mathrm{~s}, 3 \mathrm{H}$, $\left.1 \mathrm{CCH}_{3}\right) ; 0.991-0.931\left(\mathrm{~m}, 18 \mathrm{H}, 2 \mathrm{OSi}\left(\mathrm{CH}_{2} \mathrm{CH}_{3}\right)_{3}\right) ; 0.755\left(\mathrm{~s}, 3 \mathrm{H}, 1 \mathrm{CCH}_{3}\right) ; 0.663$ (qd, J = 7.7, $\left.1.3 \mathrm{~Hz}, 6 \mathrm{H}, \mathrm{OSi}\left(\mathrm{CH}_{2} \mathrm{CH}_{3}\right)_{3}\right) ; 0.590$ (q, J=8.0 Hz, $\left.6 \mathrm{H}, \mathrm{OSi}\left(\mathrm{CH}_{2} \mathrm{CH}_{3}\right)_{3}\right)$.

${ }^{13} \mathrm{C}-N M R\left(151 \mathrm{MHz}, \mathrm{CDCl}_{3}\right): \delta(\mathrm{ppm}): 137.1(\mathrm{CH}) ; 116.3(\mathrm{CH} 2) ; 80.5(\mathrm{CH}) ; 75.5(\mathrm{CH}) ; 60.2$ $\left(\mathrm{CH}_{2}\right) ; 40.6(\mathrm{C}) ; 36.6\left(\mathrm{CH}_{2}\right) ; 35.9\left(\mathrm{CH}_{2}\right) ; 23.3\left(\mathrm{CH}_{3}\right) ; 20.2\left(\mathrm{CH}_{3}\right) ; 7.1\left(\mathrm{CH}_{3}\right) ; 6.9\left(\mathrm{CH}_{3}\right) ; 5.4$ $\left(\mathrm{CH}_{2}\right) ; 4.6\left(\mathrm{CH}_{2}\right)$.

IR(neat): 3488 (br); 2955 (m); 2913 (m); 2876 (m); 1677 (w); 1640 (w); 1461 (m); 1415 (m); $1381(\mathrm{~m}) ; 1238(\mathrm{~m}) ; 1085(\mathrm{~s}) ; 1004(\mathrm{~s}) ; 908(\mathrm{w}) ; 782(\mathrm{~m}) ; 725(\mathrm{~s}) ; 672(\mathrm{~m}) \mathrm{cm}^{-1}$.

MS (ESI): $m / z(\%): 153.13$ (100), 303.23 (62), 285.22 (40), 254.24 (38), 325.21 (4), 417.32 (2) $[M+H]^{+}$.

HRMS (ESI) m/ $z$ : [M+H]+ Calcd for $\mathrm{C}_{22} \mathrm{H}_{48} \mathrm{O}_{3} \mathrm{Si}_{2}: 417.3220$; found: 417.3270 .

(5S,7S)-5-allyl-11,11-diethyl-6,6-dimethyl-7-((triethylsilyl)oxy)-2,4,10-trioxa-11silatridecane (10)

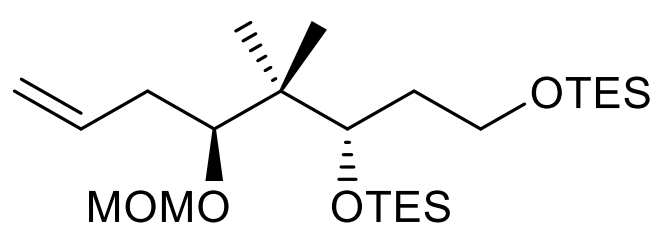

10

MOMCl $(1.3 \mathrm{~mL}, 17 \mathrm{mmol}, 3 \mathrm{eq})$ was added dropwise at $0^{\circ} \mathrm{C}$ to a solution of allylic alcohol 9 (2.36 g, $5.67 \mathrm{mmol}, 1 \mathrm{eq})$, DIPEA ( $3 \mathrm{~mL}, 17 \mathrm{mmol}, 3 \mathrm{eq}$ ) and DMAP (207 mg, $1.7 \mathrm{mmol}$, $0.3 \mathrm{eq})$ in $\mathrm{CH}_{2} \mathrm{Cl}_{2}(60 \mathrm{~mL})$. The mixture was stirred for $16 \mathrm{~h}$ at $50^{\circ} \mathrm{C}$ and then filtered on a pad of silica (pentane/Et $\mathrm{E}_{2} \mathrm{O} 10: 1$ ). The filtrate was concentrated in vacuo, the residue was again dissolved in $\mathrm{CH}_{2} \mathrm{Cl}_{2}(60 \mathrm{~mL})$ and treated with DIPEA ( $\left.3 \mathrm{~mL}, 17 \mathrm{mmol}, 3 \mathrm{eq}\right)$ and DMAP (207 mg, $1.7 \mathrm{mmol}, 0.3 \mathrm{eq})$. The mixture was cooled to $0^{\circ} \mathrm{C}, \mathrm{MOMCl}(1.3 \mathrm{~mL}, 17 \mathrm{mmol}, 3$ eq) was added dropwise and then the mixture was stirred for $20 \mathrm{~h}$ at $50^{\circ} \mathrm{C}$. Evaporation of the solvent and purification of the residue by flash chromatography (pentane/Et $2 \mathrm{O} 60: 1$ ) afforded the protected triol $10(2.4 \mathrm{~g}, 5.21 \mathrm{mmol}, 92 \%)$ as a colorless liquid.

General Data: $\mathrm{C}_{24} \mathrm{H}_{52} \mathrm{O}_{4} \mathrm{Si}_{2} ; \mathrm{FW}: 460.34$; TLC: $\mathrm{R}_{\mathrm{f}}=0.5$ (pentane/Et $2 \mathrm{O} 50: 1$ ); UV (-); Vanillin: dark blue; $[\alpha]_{D}^{20}=-20.0\left(c=0.9, \mathrm{CHCl}_{3}\right)$.

${ }^{1} \mathrm{H}-\mathrm{NMR}\left(600 \mathrm{MHz}, \mathrm{CDCl}_{3}\right): \delta(\mathrm{ppm}): 5.94-5.84\left(\mathrm{~m}, 1 \mathrm{H}, \mathrm{CH}=\mathrm{CH}_{2}\right) ; 5.10-4.96(\mathrm{~m}, 2 \mathrm{H}$, $\mathrm{CH}=\mathrm{CH}_{2}$ ); 4.64 (dd, $\left.J=17.3,6.7 \mathrm{~Hz}, 2 \mathrm{H}, \mathrm{OCH}_{2} \mathrm{OCH}_{3}\right) ; 3.72(\mathrm{~d}, J=9.0 \mathrm{~Hz}, 1 \mathrm{H}$, $\left.\mathrm{CHOCH}_{2} \mathrm{OCH}_{3}\right)$; 3.71-3.65 $\left(\mathrm{m}, 1 \mathrm{H}, 1 \mathrm{CH}_{2} \mathrm{OSi}\right) ; 3.64-3.57\left(\mathrm{~m}, 1 \mathrm{H}, 1 \mathrm{CH}_{2} \mathrm{OSi}\right) ; 3.40(\mathrm{dd}, \mathrm{J}=$ 8.4, $2.5 \mathrm{~Hz} 1 \mathrm{H}, \mathrm{CHOSi}) ; 3.37\left(\mathrm{~s}, 3 \mathrm{H}, \mathrm{OCH}_{3}\right) ; 2.35-2.26\left(\mathrm{~m}, 1 \mathrm{H}, 1 \mathrm{CH}_{2} \mathrm{CH}\right) ; 2.23-2.14(\mathrm{~m}, 1 \mathrm{H}$, $\left.1 \mathrm{CH}_{2} \mathrm{CH}\right) ; 1.72-1.60\left(\mathrm{~m}, 1 \mathrm{H}, 1 \mathrm{CH}_{2} \mathrm{CH}_{2} \mathrm{O}\right) ; 1.55-1.45\left(\mathrm{~m}, 1 \mathrm{H}, 1 \mathrm{CH}_{2} \mathrm{CH}_{2} \mathrm{O}\right) ; 1.00-0.923(\mathrm{~m}$, $\left.18 \mathrm{H}, 2 \mathrm{OSi}\left(\mathrm{CH}_{2} \mathrm{CH}_{3}\right)_{3}\right) ; 0.883\left(\mathrm{~s}, 6 \mathrm{H}, \mathrm{C}\left(\mathrm{CH}_{3}\right)_{2}\right) ; 0.674-0.544\left(\mathrm{~m}, 12 \mathrm{H}, 2 \mathrm{OSi}\left(\mathrm{CH}_{2} \mathrm{CH}_{3}\right)_{3}\right)$.

${ }^{13} \mathrm{C}-N M R\left(151 \mathrm{MHz}, \mathrm{CDCl}_{3}\right): \delta(\mathrm{ppm}): 137.0(\mathrm{CH}) ; 116.2\left(\mathrm{CH}_{2}\right) ; 98.2\left(\mathrm{CH}_{2}\right) ; 83.9(\mathrm{CH}) ; 73.9$ $(\mathrm{CH}) ; 60.4\left(\mathrm{CH}_{2}\right) ; 56.2\left(\mathrm{CH}_{3}\right) ; 43.7(\mathrm{C}) ; 35.9\left(\mathrm{CH}_{2}\right) ; 35.7\left(\mathrm{CH}_{2}\right) ; 19.9\left(\mathrm{CH}_{3}\right) ; 19.4\left(\mathrm{CH}_{3}\right) ; 7.3$ $\left(\mathrm{CH}_{3}\right) ; 6.9\left(\mathrm{CH}_{3}\right) ; 5.8\left(\mathrm{CH}_{2}\right) ; 4.6\left(\mathrm{CH}_{2}\right)$. 
IR(neat): $2954(\mathrm{~m}) ; 2911(\mathrm{~m}) ; 2876(\mathrm{~m}) ; 1640(\mathrm{w}) ; 1459(\mathrm{~m}) ; 1415(\mathrm{~m}) ; 1386(\mathrm{~m}) ; 1238(\mathrm{~m})$; 1090 (s); 1036 (s); 1004 (s); $912(\mathrm{~m}) ; 726(\mathrm{~s}) ; 673(\mathrm{~m}) \mathrm{cm}^{-1}$.

MS (ESI): $m / z(\%): 506.52$ (100), $243.17(23), 457.33(4), 461.34(<1)[M+H]^{+}$.

HRMS (ESI) $m / z$ : $[M+H]^{+}$Calcd for $\mathrm{C}_{24} \mathrm{H}_{52} \mathrm{O}_{4} \mathrm{Si}_{2}: 461.3438$; found: 461.3460.

(3S,5S,E)-5-(methoxymethoxy)-4,4-dimethyl-3-((triethylsilyl)oxy)non-7-en-1-ol (12)

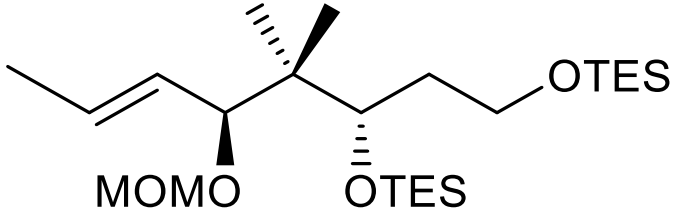

11

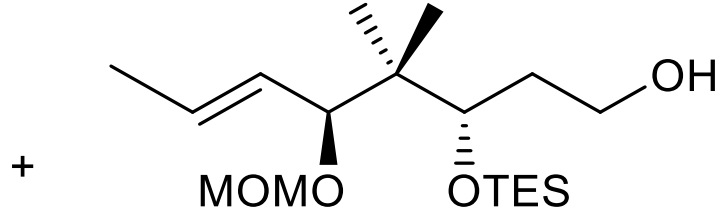

12

A solution of protected triol $10(2.3 \mathrm{~g}, 5 \mathrm{mmol}, 1 \mathrm{eq})$ and Grubbs II (212 $\mathrm{mg}, 0.250 \mathrm{mmol}$, $0.05 \mathrm{eq}$ ) in $\mathrm{MeOH}$ was stirred for $22 \mathrm{~h}$ at $60^{\circ} \mathrm{C}$. The mixture was then concentrated in vacuo and the residue was filtered on a pad of silica gel (pentane/Et ${ }_{2} \mathrm{O} 1: 1$ ). The filtrate was concentrated in vacuo affording a mixture of $12(80 \%)$ and $11(15 \%)$ as a colorless liquid, which was used in the next step without further purification. A small amount was further purified by flash chromatography (pentane/Et ${ }_{2} \mathrm{O} 60: 1$ to 3:1) for analytical purpose.

General Data (11): $\mathrm{C}_{24} \mathrm{H}_{52} \mathrm{O}_{4} \mathrm{Si}_{2} ; \mathrm{FW}: 460.34$; TLC: $\mathrm{R}_{\mathrm{f}}=0.5$ (pentane/Et $2 \mathrm{O} 50: 1$ ); UV (-); Vanillin: dark blue; $[\alpha]_{D}^{20}=+7.8\left(c=1.0, \mathrm{CHCl}_{3}\right)$.

${ }^{1} \mathrm{H}-N M R(11)\left(400 \mathrm{MHz}, \mathrm{CDCl}_{3}\right): \delta(\mathrm{ppm}): 5.64-5.53(\mathrm{~m}, 1 \mathrm{H}, \mathrm{CH}=\mathrm{CH}) ; 5.36-5.27(\mathrm{~m}, 1 \mathrm{H}$, $\mathrm{CH}=\mathrm{CH}) ; 4.66\left(\mathrm{~d}, J=6.6 \mathrm{~Hz}, 1 \mathrm{H}, 10 \mathrm{CH}_{2} \mathrm{OCH}_{3}\right) ; 4.46\left(\mathrm{~d}, J=6.6 \mathrm{~Hz}, 1 \mathrm{H}, 1 \mathrm{OCH}_{2} \mathrm{OCH}_{3}\right) ; 3.79$ (d, $\left.J=8.9 \mathrm{~Hz}, 1 \mathrm{H}, \mathrm{CHOCH}_{2} \mathrm{OCH}_{3}\right) ; 3.75-3.66\left(\mathrm{~m}, 1 \mathrm{H}, 1 \mathrm{CH}_{2} \mathrm{OSi}\right) ; 3.63$ (dd, J = 9.1, $2.2 \mathrm{~Hz}$, $1 \mathrm{H}, \mathrm{CHOSi}) ; 3.60-3.51\left(\mathrm{~m}, 1 \mathrm{H}, 1 \mathrm{CH}_{2} \mathrm{OSi}\right) ; 3.35\left(\mathrm{~s}, 3 \mathrm{H}, \mathrm{OCH}_{3}\right) ; 1.78-1.65(\mathrm{~m}, 1 \mathrm{H}$, $1 \mathrm{CH}_{2} \mathrm{CH}_{2} \mathrm{O}$ ); 1.71 (dd, $\left.J=6.4,1.5 \mathrm{~Hz}, 3 \mathrm{H}, \mathrm{CHCH}_{3}\right) ; 1.61-1.48\left(\mathrm{~m}, 1 \mathrm{H}, 1 \mathrm{CH}_{2} \mathrm{CH}_{2} \mathrm{O}\right.$ ); 0.994$0.929\left(\mathrm{~m}, 18 \mathrm{H}, 2 \mathrm{OSi}\left(\mathrm{CH}_{2} \mathrm{CH}_{3}\right)_{3}\right) ; 0.899\left(\mathrm{~s}, 3 \mathrm{H}, 1 \mathrm{CCH}_{3}\right) ; 0.848\left(\mathrm{~s}, 3 \mathrm{H}, 1 \mathrm{CCH}_{3}\right) ; 0.664-0.547$ $\left(\mathrm{m}, 12 \mathrm{H}, 2 \mathrm{OSi}\left(\mathrm{CH}_{2} \mathrm{CH}_{3}\right)_{3}\right)$.

${ }^{13}$ C-NMR (11) (100 MHz, $\left.\mathrm{CDCl}_{3}\right): \delta(\mathrm{ppm}): 130.7(\mathrm{CH}) ; 128.0(\mathrm{CH}) ; 93.5\left(\mathrm{CH}_{2}\right) ; 81.5(\mathrm{CH})$; $74.0(\mathrm{CH}) ; 60.9\left(\mathrm{CH}_{2}\right) ; 55.7\left(\mathrm{CH}_{3}\right) ; 42.7(\mathrm{C}) ; 36.0\left(\mathrm{CH}_{2}\right) ; 19.8\left(\mathrm{CH}_{3}\right) ; 19.4\left(\mathrm{CH}_{3}\right) ; 17.9\left(\mathrm{CH}_{3}\right)$; $7.3\left(\mathrm{CH}_{3}\right) ; 6.9\left(\mathrm{CH}_{3}\right) ; 5.8\left(\mathrm{CH}_{2}\right) ; 4.6\left(\mathrm{CH}_{2}\right)$.

IR(neat) (11): $2954(\mathrm{~m}) ; 2918(\mathrm{~m}) ; 2877(\mathrm{~m}) ; 1730(\mathrm{w}) ; 1671(\mathrm{w}) ; 1632(\mathrm{w}) ; 1461(\mathrm{~m}) ; 1415$ $(\mathrm{m}) ; 1379(\mathrm{~m}) ; 1239(\mathrm{~m}) ; 1095(\mathrm{~s}) ; 1033(\mathrm{~s}) ; 973(\mathrm{~m}) ; 922(\mathrm{~m}) ; 823(\mathrm{~m}) ; 726(\mathrm{~s}) \mathrm{cm}^{-1}$.

MS (ESI) (11): m/z (\%): 156.11 (100), 506.52 (75), 303.21 (25), 369.23 (18), $461.34(<1)$ $[\mathrm{M}+\mathrm{H}]^{+}$.

HRMS (ESI) $m / z$ (11): $[M+H]^{+}$Calcd for $\mathrm{C}_{24} \mathrm{H}_{53} \mathrm{O}_{4} \mathrm{Si}_{2}: 461.3482$; found: 461.3445 .

General Data (12): $\mathrm{C}_{18} \mathrm{H}_{38} \mathrm{O}_{4} \mathrm{Si} ; \mathrm{FW}: 346.25$; TLC: $\mathrm{R}_{\mathrm{f}}=0.3$ (pentane/Et $\mathrm{E}_{2} \mathrm{O}$ 3:1); UV (-); Vanillin: dark blue; $[\alpha]_{D}^{20}=+24.8\left(c=0.5, \mathrm{CHCl}_{3}\right)$.

${ }^{1} \mathrm{H}-N M R(12)\left(400 \mathrm{MHz}, \mathrm{CDCl}_{3}\right): \delta(\mathrm{ppm}): 5.65-5.54(\mathrm{~m}, 1 \mathrm{H}, \mathrm{CH}=\mathrm{CH}) ; 5.37-5.28(\mathrm{~m}, 1 \mathrm{H}$, $\mathrm{CH}=\mathrm{CH}) ; 4.66\left(\mathrm{~d}, J=6.5 \mathrm{~Hz}, 1 \mathrm{H}, 1 \mathrm{OCH} \mathrm{OCH}_{3}\right) ; 4.46\left(\mathrm{~d}, J=6.5 \mathrm{~Hz}, 1 \mathrm{H}, 1 \mathrm{OCH}_{2} \mathrm{OCH}_{3}\right) ; 3.78$ $\left(\mathrm{d}, J=9.1 \mathrm{~Hz}, 1 \mathrm{H}, \mathrm{CHOCH}_{2} \mathrm{OCH}_{3}\right) ; 3.78-3.71\left(\mathrm{~m}, 1 \mathrm{H}, 1 \mathrm{CH}_{2} \mathrm{OSi}\right) ; 3.68$ (dd, $J=8.7,2.6 \mathrm{~Hz}$, $1 \mathrm{H}, \mathrm{CHOSi}) ; 3.66-3.59\left(\mathrm{~m}, 1 \mathrm{H}, 1 \mathrm{CH}_{2} \mathrm{OSi}\right) ; 3.35\left(\mathrm{~s}, 3 \mathrm{H}, \mathrm{OCH}_{3}\right) ; 1.84-1.60(\mathrm{~m}, 1 \mathrm{H}$, $1 \mathrm{CH}_{2} \mathrm{CH}_{2} \mathrm{O}$ ); 1.72 (dd, $\left.J=6.4,1.6 \mathrm{~Hz}, 3 \mathrm{H}, \mathrm{CHCH}_{3}\right) ; 1.66-1.55\left(\mathrm{~m}, 1 \mathrm{H}, 1 \mathrm{CH}_{2} \mathrm{CH}_{2} \mathrm{O}\right.$ ); 0.963 $\left(\mathrm{t}, J=7.9 \mathrm{~Hz}, 9 \mathrm{H}, \mathrm{OSi}\left(\mathrm{CH}_{2} \mathrm{CH}_{3}\right)_{3}\right) ; 0.915\left(\mathrm{~s}, 3 \mathrm{H}, 1 \mathrm{CCH}_{3}\right) ; 0.867$ (s, 3H, $\left.1 \mathrm{CCH}_{3}\right) ; 0.630$ (q, $\left.6 \mathrm{H}, J=8.0 \mathrm{~Hz}, \mathrm{OSi}\left(\mathrm{CH}_{2} \mathrm{CH}_{3}\right)_{3}\right)$. 
${ }^{13} \mathrm{C}$-NMR (12) $\left(100 \mathrm{MHz}, \mathrm{CDCl}_{3}\right): \delta(\mathrm{ppm}): 131.1(\mathrm{CH}) ; 127.9(\mathrm{CH}) ; 93.5\left(\mathrm{CH}_{2}\right) ; 81.7(\mathrm{CH})$; 74.6 $(\mathrm{CH}) ; 61.1\left(\mathrm{CH}_{2}\right) ; 55.8\left(\mathrm{CH}_{3}\right) ; 42.6(\mathrm{C}) ; 35.7\left(\mathrm{CH}_{2}\right) ; 20.0\left(\mathrm{CH}_{3}\right) ; 19.3\left(\mathrm{CH}_{3}\right) ; 18.0\left(\mathrm{CH}_{3}\right)$; $7.3\left(\mathrm{CH}_{3}\right) ; 5.7\left(\mathrm{CH}_{2}\right)$.

IR(neat) (12): 3386 (br); 2954 (m); $2877(\mathrm{~m}) ; 1669$ (w); 1465 (m); 1415 (m); $1382(\mathrm{~m}) ; 1238$ $(\mathrm{m}) ; 1146(\mathrm{~m}) ; 1091(\mathrm{~m}) ; 1033(\mathrm{~s}) ; 972(\mathrm{~m}) ; 921(\mathrm{~m}) ; 840(\mathrm{~m}) ; 727(\mathrm{~s}) \mathrm{cm}^{-1}$.

MS (ESI) (12): $\mathrm{m} / z(\%): 156.11$ (100), 158.09 (48), 369.24 (42) [M+Na] ${ }^{+}, 189.12(28), 506.25$ (5), $347.26(<1)[M+H]^{+}$.

HRMS (ESI) $\mathrm{m} / z$ (12): $[\mathrm{M}+\mathrm{H}]^{+}$Calcd for $\mathrm{C}_{18} \mathrm{H}_{39} \mathrm{O}_{4} \mathrm{Si}: 347.2618$; found: 347.2570 .

(3S,5S,E)-5-(methoxymethoxy)-4,4-dimethyl-3-((triethylsilyl)oxy)oct-6-enal (13)

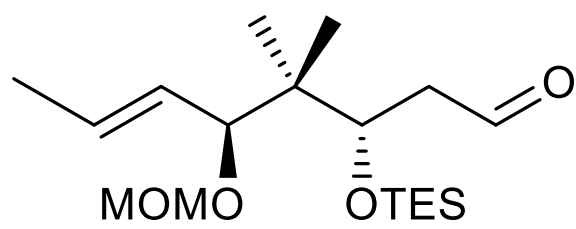

13

DMSO (0.780 mL, $11 \mathrm{mmol}, 2 \mathrm{eq})$ in $\mathrm{CH}_{2} \mathrm{Cl}_{2}(5 \mathrm{~mL})$ was added dropwise to a solution of oxalyl chloride $(0.644 \mathrm{~mL}, 7.5 \mathrm{mmol}, 1.5 \mathrm{eq})$ in $\mathrm{CH}_{2} \mathrm{Cl}_{2}(20 \mathrm{~mL})$ at $-78^{\circ} \mathrm{C}$. The mixture was stirred for 10 min at $-78^{\circ} \mathrm{C}$ and then the crude $\mathbf{1 1}+12$ dissolved in $\mathrm{CH}_{2} \mathrm{Cl}_{2}(5 \mathrm{~mL})$ was added dropwise. The reaction was stirred for $1 \mathrm{~h}$ at $-78^{\circ} \mathrm{C}$, quenched by dropwise addition of $\mathrm{NEt}_{3}$ ( $3.5 \mathrm{~mL}, 25 \mathrm{mmol}, 5 \mathrm{eq})$ and then warmed to room temperature over $45 \mathrm{~min} . \mathrm{H}_{2} \mathrm{O}(30 \mathrm{~mL})$ was added and the layers were separated. The aqueous phase was extracted with $\mathrm{CH}_{2} \mathrm{Cl}_{2}$ $(3 \times 20 \mathrm{~mL})$ and the combined organic extracts were dried over $\mathrm{Na}_{2} \mathrm{SO}_{4}$, filtered and concentrated in vacuo. Purification of the residue by flash chromatography (pentane/Et ${ }_{2} \mathrm{O}$ 10:1) afforded aldehyde $13(1.33 \mathrm{~g}, 3.86 \mathrm{mmol}, 77 \%)$ as a colorless liquid.

General Data: $\mathrm{C}_{18} \mathrm{H}_{36} \mathrm{O}_{4} \mathrm{Si} ; \mathrm{FW}: 344.24$; TLC: $\mathrm{R}_{\mathrm{f}}=0.3$ (pentane/Et $\mathrm{E}_{2} \mathrm{O}$ 10:1); UV (-); Vanillin: grey; $[\alpha]_{D}^{20}=+33.10\left(c=1.0, \mathrm{CHCl}_{3}\right)$.

${ }^{1} \mathrm{H}-\mathrm{NMR}\left(600 \mathrm{MHz}, \mathrm{CDCl}_{3}\right): \delta(\mathrm{ppm}): 9.80(\mathrm{dd}, J=3.0,1.4 \mathrm{~Hz}, 1 \mathrm{H}, \mathrm{CHO}) ; 5.59-5.52(\mathrm{~m}, 1 \mathrm{H}$, $\mathrm{CH}=\mathrm{CH}) ; 5.38-5.32(\mathrm{~m}, 1 \mathrm{H}, \mathrm{CH}=\mathrm{CH}) ; 4.65\left(\mathrm{~d}, J=6.7 \mathrm{~Hz}, 1 \mathrm{H}, 1 \mathrm{OCH}_{2} \mathrm{OCH}_{3}\right) ; 4.45(\mathrm{~d}, J=6.7$ $\left.\mathrm{Hz}, 1 \mathrm{H}, 1 \mathrm{OCH}_{2} \mathrm{OCH}_{3}\right) ; 4.18(\mathrm{dd}, J=6.8,3.8 \mathrm{~Hz}, 1 \mathrm{H}, \mathrm{CHOSi}) ; 3.73(\mathrm{~d}, J=9.3 \mathrm{~Hz}, 1 \mathrm{H}$, $\mathrm{CHOCH}_{2} \mathrm{OCH}_{3}$ ); 3.34 (s, 3H, OCH$H_{3}$; 2.62 (ddd, $J=16.7,3.9,1.4 \mathrm{~Hz}, 1 \mathrm{H}, 1 \mathrm{CH}_{2} \mathrm{CO}$ ); 2.54 (ddd, $\left.J=16.7,6.9,3.0 \mathrm{~Hz}, 1 \mathrm{H}, 1 \mathrm{CH}_{2} \mathrm{CO}\right) ; 1.72\left(\mathrm{dd}, J=6.4,1.6 \mathrm{~Hz}, 3 \mathrm{H}, \mathrm{CHCH}_{3}\right) ; 0.943(\mathrm{t}, J$ $\left.=7.9 \mathrm{~Hz}, 9 \mathrm{H}, \mathrm{OSi}\left(\mathrm{CH}_{2} \mathrm{CH}_{3}\right)_{3}\right) ; 0.895\left(\mathrm{~s}, 3 \mathrm{H}, 1 \mathrm{CCH}_{3}\right) ; 0.865\left(\mathrm{~s}, 3 \mathrm{H}, 1 \mathrm{CCH}_{3}\right) ; 0.592(\mathrm{q}, J=8.0$ $\left.\mathrm{Hz}, 6 \mathrm{H}, \mathrm{OSi}\left(\mathrm{CH}_{2} \mathrm{CH}_{3}\right)_{3}\right)$.

${ }^{13} \mathrm{C}-\mathrm{NMR}\left(151 \mathrm{MHz}, \mathrm{CDCl}_{3}\right): \delta(\mathrm{ppm}): 202.5(\mathrm{C}=\mathrm{O}) ; 131.4(\mathrm{CH}) ; 127.9(\mathrm{CH}) ; 93.5\left(\mathrm{CH}_{2}\right)$; $81.9(\mathrm{CH}) ; 72.2(\mathrm{CH}) ; 55.8\left(\mathrm{CH}_{3}\right) ; 48.2\left(\mathrm{CH}_{2}\right) ; 42.6(\mathrm{C}) ; 20.3\left(\mathrm{CH}_{3}\right) ; 19.8\left(\mathrm{CH}_{3}\right) ; 17.9\left(\mathrm{CH}_{3}\right)$; $7.1\left(\mathrm{CH}_{3}\right) ; 5.5\left(\mathrm{CH}_{2}\right)$.

IR(neat): $3075(\mathrm{w}) ; 2955(\mathrm{~m}) ; 2913(\mathrm{~m}) ; 2876(\mathrm{~m}) ; 1677(\mathrm{~m}) ; 1640(\mathrm{~m}) ; 1461(\mathrm{~m}) ; 1415(\mathrm{~m})$; $1381(\mathrm{w}) ; 1238(\mathrm{~m}) ; 1085(\mathrm{~s}) ; 1004(\mathrm{~s}) ; 974(\mathrm{~m}) ; 908(\mathrm{~m}) ; 782(\mathrm{~m}) ; 725(\mathrm{~s}) \mathrm{cm}^{-1}$.

MS (ESI): $m / z(\%): 362.27(100)[M+N a]^{+}, 367.22(76), 345.24(28)[M+H]^{+}, 369.24(16)$, $285.22(7)$.

HRMS (ESI) $\mathrm{m} / z:[\mathrm{M}+\mathrm{H}]^{+}$Calcd for $\mathrm{C}_{18} \mathrm{H}_{36} \mathrm{O}_{4} \mathrm{Si}: 345.2461$; found: 345.2436 . 
(5S,7S)-9,9-diethyl-7-((Z)-3-iodoallyl)-6,6-dimethyl-5-((E)-prop-1-en-1-yl)-2,4,8-trioxa-

9-silaundecane (14)

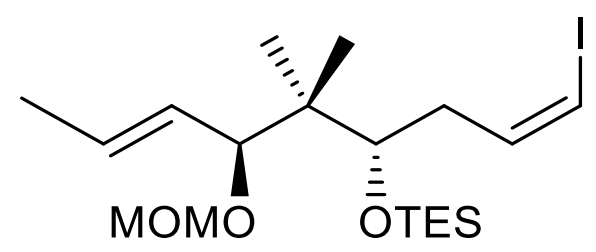

14

NaHMDS ( $1 \mathrm{M}$ in THF, $4.2 \mathrm{~mL}, 4.2 \mathrm{mmol}, 1.5 \mathrm{eq}$ ) was added dropwise at $0^{\circ} \mathrm{C}$ to a solution of IMePPh 3 ( $2.22 \mathrm{~g}, 4.2 \mathrm{mmol}, 1.5 \mathrm{eq})$ in THF $(30 \mathrm{~mL})$. The red solution was stirred for 10 $\mathrm{min}$ at room temperature and then cooled to $-78^{\circ} \mathrm{C}$. DMPU $(2.5 \mathrm{~mL}, 20.93 \mathrm{mmol}, 7.5 \mathrm{eq})$ was added dropwise, followed by aldehyde $13(960 \mathrm{mg}, 2.79 \mathrm{mmol}, 1 \mathrm{eq})$ in THF $(7 \mathrm{~mL})$. The mixture was stirred for $1 \mathrm{~h}$ at $-78^{\circ} \mathrm{C}$ and 30 min at room temperature. Saturated $\mathrm{NH}_{4} \mathrm{Cl}$ solution $(30 \mathrm{~mL})$ was added and the aqueous phase was extracted with $\mathrm{Et}_{2} \mathrm{O}(3 \times 25 \mathrm{~mL})$. The combined organic layers were dried over $\mathrm{Na}_{2} \mathrm{SO}_{4}$, filtered and concentrated in vacuo. The residue was purified by flash chromatography (pentane/Et $\mathrm{t}_{2} \mathrm{O} 100: 1$ ) giving the $Z$ lodide $14(875 \mathrm{mg}, 1.87 \mathrm{mmol}, 67 \%)$ as a slightly yellow liquid.

General Data: $\mathrm{C}_{19} \mathrm{H}_{37} \mathrm{IO}_{3} \mathrm{Si}$; FW: 468.16; TLC: $\mathrm{R}_{\mathrm{f}}=0.25$ (pentane/ $\mathrm{Et}_{2} \mathrm{O}$ 100:1); UV (-); Vanillin: black; $[\alpha]_{D}^{20}=+7.8\left(c=1.0, \mathrm{CHCl}_{3}\right)$.

${ }^{1} \mathrm{H}-\mathrm{NMR}\left(600 \mathrm{MHz}, \mathrm{CDCl}_{3}\right): \delta(\mathrm{ppm}): 6.32(\mathrm{q}, J=6.8 \mathrm{~Hz}, 1 \mathrm{H}, \mathrm{CH}=\mathrm{CHI}) ; 6.22(\mathrm{~m}, 1 \mathrm{H}$, $\mathrm{CH}=\mathrm{CH})$; 5.72-5.63 (m, $1 \mathrm{H}, \mathrm{CH}=\mathrm{CH}) ; 5.38-5.29(\mathrm{~m}, 1 \mathrm{H}, \mathrm{CH}=\mathrm{CH}) ; 4.68(\mathrm{~d}, J=6.5 \mathrm{~Hz}, 1 \mathrm{H}$, $\left.1 \mathrm{OCH}_{2} \mathrm{OCH}_{3}\right) ; 4.48\left(\mathrm{~d}, J=6.5 \mathrm{~Hz}, 1 \mathrm{H}, 1 \mathrm{OCH}_{2} \mathrm{OCH}_{3}\right) ; 3.82(\mathrm{~d}, J=9.1 \mathrm{~Hz}, 1 \mathrm{H}$, $\left.\mathrm{CHOCH}_{2} \mathrm{OCH}_{3}\right) ; 3.72(\mathrm{dd}, J=6.7,4.1 \mathrm{~Hz}, 1 \mathrm{H}, \mathrm{CHOSi}) ; 3.36\left(\mathrm{~s}, 3 \mathrm{H}, \mathrm{OCH}_{3}\right) ; 2.38-2.21(\mathrm{~m}$, $\left.2 \mathrm{H}, \mathrm{CH}_{2} \mathrm{CH}\right) ; 1.73\left(\mathrm{dd}, J=6.1,1.4 \mathrm{~Hz}, 3 \mathrm{H}, \mathrm{CHCH}_{3}\right) ; 0.957$ (t, $\left.9 \mathrm{H}, J=8.0, \mathrm{OSi}_{2}\left(\mathrm{CH}_{2} \mathrm{CH}_{3}\right)_{3}\right)$; $0.929\left(\mathrm{~s}, 3 \mathrm{H}, 1 \mathrm{CCH}_{3}\right) ; 0.867\left(\mathrm{~s}, 3 \mathrm{H}, 1 \mathrm{CCH}_{3}\right) ; 0.599\left(\mathrm{q}, J=8.0 \mathrm{~Hz}, 6 \mathrm{H}, \mathrm{OSi}\left(\mathrm{CH}_{2} \mathrm{CH}_{3}\right)_{3}\right)$.

${ }^{13} \mathrm{C}$-NMR $\left(151 \mathrm{MHz}, \mathrm{CDCl}_{3}\right)$ : $\delta(\mathrm{ppm}): 140.2(\mathrm{CH}) ; 131.4(\mathrm{CH}) ; 127.8(\mathrm{CH}) ; 93.6\left(\mathrm{CH}_{2}\right) ; 82.8$ $(\mathrm{CH}) ; 81.7(\mathrm{CH}) ; 75.7(\mathrm{CH}) ; 55.8\left(\mathrm{CH}_{3}\right) ; 43.1(\mathrm{C}) ; 38.8\left(\mathrm{CH}_{2}\right) ; 19.9\left(\mathrm{CH}_{3}\right) ; 19.6\left(\mathrm{CH}_{3}\right) ; 18.1$ $\left(\mathrm{CH}_{3}\right) ; 7.3\left(\mathrm{CH}_{3}\right) ; 5.7\left(\mathrm{CH}_{2}\right)$.

IR(neat): 3069 (w); 2980 (s); $2884(\mathrm{~m}) ; 1668$ (w); 1462 (m); 1382 (m); 1240 (m); 1147 (m); $1088(\mathrm{~s}) ; 1036(\mathrm{~s}) ; 970(\mathrm{~m}) ; 922(\mathrm{~m}) ; 820(\mathrm{w}) ; 724(\mathrm{~s}) ; 572(\mathrm{w}) \mathrm{cm}^{-1}$.

MS (ESI): m/z (\%): 506.53 (100), 507.53 (45), 504.51 (22), 469.16 (8) [M+H] $]^{+}, 457.16$ (5), 396.31 (4).

HRMS (ESI) m/z: $[M+H]^{+}$Calcd for $\mathrm{C}_{19} \mathrm{H}_{38} \mathrm{IO}_{3} \mathrm{Si}: 469.1635$; found: 469.1682 .

tert-Butyl ( $R$ )-3-hydroxypent-4-enoate (15)<smiles>C=C[C@H](O)CC(=O)OCCCC</smiles>

(R)-15 (99:1 er) $\quad 47 \%$<smiles>C=C[C@@H](CC(=O)OCCC)OC(C)=O</smiles>

OAc-(S)-15 48\%

Vinyl acetate ( $16 \mathrm{~mL}, 174.18 \mathrm{mmol}, 3 \mathrm{eq})$ was added to a solution of racemic tert-Butyl 3hydroxypent-4-enoate $(10 \mathrm{~g}, 58.06 \mathrm{mmol}, 1 \mathrm{eq})$ in pentane $(200 \mathrm{~mL})$. Then Amano Lipase PS 
$(6 \mathrm{~g})$ and $4 \hat{\mathrm{A}}$ molecular sieves $(9.5 \mathrm{~g})$ were added and the mixture was stirred for $24 \mathrm{~h}$ at $30^{\circ} \mathrm{C}$. The solids were filtered on paper and washed with $\mathrm{Et}_{2} \mathrm{O}$; the filtrate was concentrated in vacuo and the residue purified by flash chromatography (pentane/Et2O 6:1 to 2:1) to afford $(\boldsymbol{R})-15(4.71 \mathrm{~g}, 27.35 \mathrm{mmol}, 47 \%, 99: 1 \mathrm{er})$ as a colorless liquid and OAc-(S)-15 (6.04 g, $28.22 \mathrm{mmol}, 48 \%)$.

General Data: $\mathrm{C}_{9} \mathrm{H}_{16} \mathrm{O}_{3}$; FW: 172.11; TLC: $\mathrm{R}_{\mathrm{f}}=0.35$ (pentane/Et2O 2:1); UV (-); Vanillin: light blue; $[\alpha]_{D}^{20}=+8.9\left(c=0.55, \mathrm{CHCl}_{3}\right)$.

${ }^{1} \mathrm{H}-\mathrm{NMR}\left(400 \mathrm{MHz}, \mathrm{CDCl}_{3}\right): \delta(\mathrm{ppm}): 5.89-5.80\left(\mathrm{~m}, 1 \mathrm{H}, \mathrm{CH}=\mathrm{CH}_{2}\right) ; 5.29(\mathrm{dt}, J=17.2,1.5 \mathrm{~Hz}$, $\left.1 \mathrm{H}, 1 \mathrm{CH}=\mathrm{CH}_{2}\right) ; 5.13\left(\mathrm{dt}, J=10.5,1.4 \mathrm{~Hz}, 1 \mathrm{H}, 1 \mathrm{CH}=\mathrm{CH}_{2}\right) ; 4.49-4.43(\mathrm{~m}, 1 \mathrm{H}, \mathrm{CHOH}) ; 2.89$ (s, $1 \mathrm{H}, \mathrm{CHOH}$; 2.50 (dd, $J=16.1,4.0 \mathrm{~Hz}, 1 \mathrm{H}, 1 \mathrm{CH} \mathrm{HH}_{2}$; 2.41 (dd, $J=16.1,8.2 \mathrm{~Hz}, 1 \mathrm{H}$, $\left.\left.1 \mathrm{CH}_{2} \mathrm{CH}\right) ; 1.45\left(\mathrm{~s}, 9 \mathrm{H}, \mathrm{OC}\left(\mathrm{CH}_{3}\right)_{3}\right)\right)$.

${ }^{13} \mathrm{C}-N M R\left(100 \mathrm{MHz}_{\mathrm{CDCl}}\right)$ : $\delta$ (ppm): $171.8(\mathrm{C}=\mathrm{O}) ; 139.0(\mathrm{CH}) ; 115.3\left(\mathrm{CH}_{2}\right) ; 81.6(\mathrm{C}) ; 69.2$ $(\mathrm{CH}) ; 42.2\left(\mathrm{CH}_{2}\right) ; 28.2\left(\mathrm{C}\left(\mathrm{CH}_{3}\right) 3\right)$.

IR (neat): 3438 (br); $2980(\mathrm{~m}) ; 2933(\mathrm{w}) ; 1726(\mathrm{~s}) ; 1367(\mathrm{~m}) ; 1152(\mathrm{~s}) ; 922(\mathrm{~m}) \mathrm{cm}^{-1}$.

MS (ESI): $m / z(\%): 173.12(100)[M+H]^{+}, 117.05(96), 190.14$ (43) $[M+N a]^{+}, 195.10$ (23), $367.21(18)$.

HRMS (ESI) $m / z:[M+H]^{+}$Calcd for $\mathrm{C}_{9} \mathrm{H}_{17} \mathrm{O}_{3}:$ 173.1178; found: 173.1183 .

tert-Butyl (R)-3-Methoxypent-4-enoate (16)<smiles>C=C[C@H](CC(=O)OCCC)OC</smiles>

16

Proton Sponge ${ }^{\circledR}(17.5 \mathrm{~g}, 81.86 \mathrm{mmol}, 3 \mathrm{eq})$ and trimethyloxonium tetrafluoroborate $(8.08 \mathrm{~g}$, $54.58 \mathrm{mmol}, 2 \mathrm{eq})$ were added to a solution of $(\boldsymbol{R})-15(4.7 \mathrm{~g}, 27.29 \mathrm{mmol}, 1 \mathrm{eq})$ in $\mathrm{CH}_{2} \mathrm{Cl}_{2}$ $(115 \mathrm{~mL})$ and the mixture was stirred for $3 \mathrm{~h}$ at room temperature. The reaction mixture was then filtered through a pad of Celite and the filtrate was washed with saturated aqueous solution of $\mathrm{NaHSO}_{4}$ to remove Proton Sponge. The aqueous phase was extracted with $\mathrm{CH}_{2} \mathrm{Cl}_{2}$ and the organic extracts were dried over $\mathrm{Na}_{2} \mathrm{SO}_{4}$, filtered and concentrated in vacuo. The residue was purified by silica gel chromatography (pentane/Et $2 \mathrm{O} 20: 1$, then 2:1) to give $16(3.91 \mathrm{~g}, 21.03 \mathrm{mmol}, 77 \%)$ as a colorless liquid and unreacted alcohol (517 mg, 3.00 mmol, 11\%).

General Data: $\mathrm{C}_{10} \mathrm{H}_{18} \mathrm{O}_{3}$; FW: 186.13; TLC: $\mathrm{R}_{\mathrm{f}}=0.30$ (pentane/Et $2 \mathrm{O} 20: 1$ ); UV (-); Vanillin: dark blue; $[\alpha]_{D}^{20}=+1.1\left(c=0.55, \mathrm{CHCl}_{3}\right)$.

${ }^{1} \mathrm{H}-\mathrm{NMR}\left(400 \mathrm{MHz}, \mathrm{CDCl}_{3}\right): \delta(\mathrm{ppm}): 5.72-5.65\left(\mathrm{~m}, 1 \mathrm{H}, \mathrm{CH}=\mathrm{CH}_{2}\right) ; 5.27(\mathrm{dt}, J=17.1,1.4 \mathrm{~Hz}$, $\left.1 \mathrm{H}, 1 \mathrm{CH}=\mathrm{CH}_{2}\right) ; 5.21\left(\mathrm{dt}, J=10.5,1.2 \mathrm{~Hz}, 1 \mathrm{H}, 1 \mathrm{CH}=\mathrm{CH}_{2}\right) ; 4.00-3.93\left(\mathrm{~m}, 1 \mathrm{H}, \mathrm{CHOCH}_{3}\right) ; 3.28$ $\left(\mathrm{s}, 3 \mathrm{H}, \mathrm{OCH}_{3}\right) ; 2.51\left(\mathrm{dd}, J=15.0,8.1 \mathrm{~Hz}, 1 \mathrm{H}, 1 \mathrm{CH}_{2} \mathrm{CH}\right) ; 2.36(\mathrm{dd}, J=15.0,5.7 \mathrm{~Hz}, 1 \mathrm{H}$, $\left.\left.1 \mathrm{CH}_{2} \mathrm{CH}\right) ; 1.44\left(\mathrm{~s}, 9 \mathrm{H}, \mathrm{OC}\left(\mathrm{CH}_{3}\right)_{3}\right)\right)$.

${ }^{13} \mathrm{C}-N M R\left(100 \mathrm{MHz}, \mathrm{CDCl}_{3}\right)$ : $\delta(\mathrm{ppm}): 170.3(\mathrm{C}=\mathrm{O}) ; 137.3(\mathrm{CH}) ; 117.8\left(\mathrm{CH}_{2}\right) ; 80.7(\mathrm{C}) ; 79.5$ $(\mathrm{CH}) ; 56.6\left(\mathrm{CH}_{3}\right) ; 42.3\left(\mathrm{CH}_{2}\right) ; 28.2\left(\mathrm{C}\left(\mathrm{CH}_{3}\right) 3\right)$.

IR (neat): $2979(\mathrm{~m}) ; 2927(\mathrm{~m}) ; 1732(\mathrm{~s}) ; 1457(\mathrm{~m}) ; 1392(\mathrm{~m}) ; 1367(\mathrm{~m}) ; 1280(\mathrm{~m}) ; 1254(\mathrm{~m})$; $1156(\mathrm{~s}) ; 1102(\mathrm{~s}) ; 1018(\mathrm{w}) ; 991(\mathrm{~m}) ; 928(\mathrm{~m}) ; 845(\mathrm{~m}) ; 765(\mathrm{~m}) \mathrm{cm}^{-1}$.

MS (ESI): $m / z(\%): 131.07$ (100), $113.06(25), 279.17(15), 187.13(9)[M+H]^{+}, 252.23(6)$

HRMS (ESI) $\mathrm{m} / \mathrm{z}$ : $[\mathrm{M}+\mathrm{H}]^{+}$Calcd for $\mathrm{C}_{10} \mathrm{H}_{19} \mathrm{O}_{3}$ : 187.1334 ; found: 187.1336 . 
tert-butyl (R)-3-methoxy-4-oxobutanoate (17)<smiles>CCCCC(=O)C[C@@H](C=O)OC</smiles>

17

A stream of $\mathrm{O}_{3}$ in $\mathrm{O}_{2}$ was bubbled through a solution of $16(3.23 \mathrm{~g}, 17.37 \mathrm{mmol}, 1 \mathrm{eq})$ in $\mathrm{CH}_{2} \mathrm{Cl}_{2}(75 \mathrm{~mL})$ and $\mathrm{MeOH}(15 \mathrm{~mL})$ at $-78^{\circ} \mathrm{C}$ until the blue color of the solution persisted. Then $\mathrm{O}_{2}$ was bubbled for $10 \mathrm{~min}$ and $\mathrm{PPh}_{3}(5.47 \mathrm{~g}, 20.84 \mathrm{mmol}, 1.2 \mathrm{eq})$ was added. The mixture was warmed to room temperature and stirred for $2 \mathrm{~h}$. The solvents were removed in vacuo and the residue was purified by flash chromatography (pentane/Et2O 2:1) to afford aldehyde 17 (2.91 g, $15.5 \mathrm{mmol}, 89 \%)$ as a colorless liquid.

General Data: $\mathrm{C}_{9} \mathrm{H}_{16} \mathrm{O}_{4}$; FW: 188.10; TLC: $\mathrm{R}_{\mathrm{f}}=0.25$ (pentane/Et $2 \mathrm{O} 2: 1$ ); UV (-); Vanillin: yellow; $[\alpha]_{D}^{20}=+30.08\left(c=1.25, \mathrm{CHCl}_{3}\right)$.

${ }^{1} \mathrm{H}-\mathrm{NMR}\left(600 \mathrm{MHz}, \mathrm{CDCl}_{3}\right): \delta$ (ppm): 9.76 (s, $\left.1 \mathrm{H}, \mathrm{CHO}\right) ; 3.93-3.89\left(\mathrm{~m}, 1 \mathrm{H}, \mathrm{CHOCH}_{3}\right) ; 3.50$ (s, 3H, OCH$) ; 2.69$ (dd, $J=16.2,4.8 \mathrm{~Hz}, 1 \mathrm{H}, 1 \mathrm{CH}_{2} \mathrm{CH}$ ); 2.60 (dd, $J=16.1,6.6 \mathrm{~Hz}, 1 \mathrm{H}$, $\left.\left.1 \mathrm{CH}_{2} \mathrm{CH}\right) ; 1.44\left(\mathrm{~s}, 9 \mathrm{H}, \mathrm{OC}\left(\mathrm{CH}_{3}\right)_{3}\right)\right)$.

${ }^{13} \mathrm{C}-N M R\left(151 \mathrm{MHz}, \mathrm{CDCl}_{3}\right): \delta(\mathrm{ppm}): 202.1(\mathrm{CHO}) ; 169.3(\mathrm{C}=\mathrm{O}) ; 82.2(\mathrm{C}) ; 81.8(\mathrm{CH}) ; 58.8$ $\left(\mathrm{CH}_{3}\right) ; 37.1\left(\mathrm{CH}_{2}\right) ; 28.2\left(\mathrm{C}\left(\mathrm{CH}_{3}\right) 3\right)$.

IR(neat): $2979(\mathrm{~m}) ; 2933(\mathrm{~m}) ; 2832(\mathrm{w}) ; 1729(\mathrm{~s}) ; 1456(\mathrm{w}) ; 1368(\mathrm{~m}) ; 1256(\mathrm{~m}) ; 1113$ (s); $1076(\mathrm{~m}) ; 953(\mathrm{w}) ; 846(\mathrm{w}) \mathrm{cm}^{-1}$.

MS (El, 70eV): m/z (\%): $189.11(100)[M+H]^{+}, 187.10(23), 188.10(23)\left[M^{+}, 190.09\right.$ (22).

HRMS (El, 70eV) m/z: [M+H] ${ }^{+}$Calcd for $\mathrm{C}_{9} \mathrm{H}_{17} \mathrm{O}_{4}$ : 189.1121 ; found: 189.1116 .

tert-butyl (R,E)-3-methoxy-7-(triisopropylsilyl)hept-4-en-6-ynoate (19)

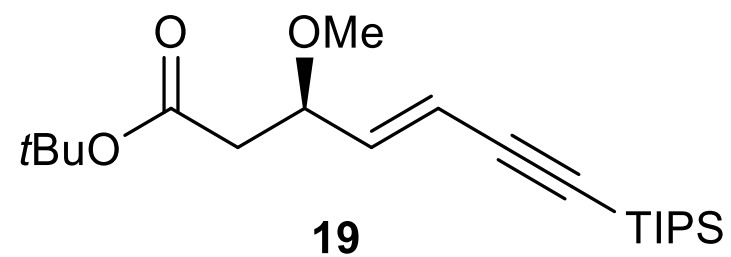

$n$ BuLi 2.5 $\mathrm{M}$ in hexane $(8 \mathrm{~mL}, 20.05 \mathrm{mmol}, 1.3 \mathrm{eq}$ ) was added dropwise to a suspension of propargyl triphenylphosphonium bromide ${ }^{18^{[4]}}(9.96 \mathrm{~g}, 18.5 \mathrm{mmol}, 1.2 \mathrm{eq})$ in THF $(100 \mathrm{~mL})$ at $-78^{\circ} \mathrm{C}$. The red solution was stirred at $0^{\circ} \mathrm{C}$ for $30 \mathrm{~min}$ and then aldehyde $17(2.9 \mathrm{~g}, 15.42$ $\mathrm{mmol}, 1 \mathrm{eq})$ in THF $(20 \mathrm{~mL})$ was added dropwise. The mixture was warmed to room temperature and stirred for $30 \mathrm{~min}$. The reaction was quenched by addition of saturated aqueous $\mathrm{NH}_{4} \mathrm{Cl}$ solution $(100 \mathrm{~mL})$ and the layers were separated. The aqueous phase was extracted with $\mathrm{Et}_{2} \mathrm{O}(3 \times 80 \mathrm{~mL})$ and the combined organic extracts were dried over $\mathrm{Na}_{2} \mathrm{SO}_{4}$, filtered and concentrated in vacuo. Purification of the residue by flash chromatography (pentane/Et2O 40:1 to 30:1) gave E-19 (3.38 g, $9.25 \mathrm{mmol}, 60 \%)$ and Z-19 (1.25 g, 3.42 $\mathrm{mmol}, 22 \%)$.

General Data: $\mathrm{C}_{21} \mathrm{H}_{38} \mathrm{O}_{3} \mathrm{Si}$; FW: 366.26; TLC: $\mathrm{R}_{\mathrm{f}}=0.45$ (pentane/Et $2 \mathrm{O} 20: 1$ ); UV (+); Vanillin: brown; $[\alpha]_{D}^{20}=+8.2\left(c=0.5, \mathrm{CHCl}_{3}\right)$.

1H-NMR (400 MHz, CDCl 3 ): $\delta(p p m): 6.01$ (dd, $J=15.8,7.2 \mathrm{~Hz}, 1 \mathrm{H}, \mathrm{CH}=\mathrm{CH}) ; 5.76$ (dd, $J=$ 15.9, $1.0 \mathrm{~Hz}, 1 \mathrm{H}, \mathrm{CH}=\mathrm{CH}) ; 4.04-3.98\left(\mathrm{~m}, 1 \mathrm{H}, \mathrm{CHOCH}_{3}\right) ; 3.30\left(\mathrm{~s}, 3 \mathrm{H}, \mathrm{OCH}_{3}\right) ; 2.50$ (dd, $J=$ $\left.14.9,7.9 \mathrm{~Hz}, 1 \mathrm{H}, 1 \mathrm{CH}_{2} \mathrm{CH}\right) ; 2.37\left(\mathrm{dd}, J=14.9,5.6 \mathrm{~Hz}, 1 \mathrm{H}, 1 \mathrm{CH}_{2} \mathrm{CH}\right) ; 1.44(\mathrm{~s}, 9 \mathrm{H}$, $\left.\left.\mathrm{OC}\left(\mathrm{CH}_{3}\right)_{3}\right)\right) ; 1.07\left(\mathrm{~s}, 21 \mathrm{H}, \mathrm{Si}\left(\mathrm{CH}\left(\mathrm{CH}_{3}\right)_{3}\right)\right.$. 
${ }^{13} \mathrm{C}-N M R\left(100 \mathrm{MHz}_{\mathrm{CDCl}}\right): \delta(\mathrm{ppm}): 169.9(\mathrm{C}=\mathrm{O}) ; 142.5(\mathrm{CH}) ; 112.9(\mathrm{CH}) ; 104.6(\mathrm{C}) ; 92.2$ $(\mathrm{C}) ; 81.0(\mathrm{C}) ; 78.5(\mathrm{CH}) ; 57.1\left(\mathrm{CH}_{3}\right) ; 42.0\left(\mathrm{CH}_{3}\right) ; 28.2\left(\mathrm{C}\left(\mathrm{CH}_{3}\right) 3\right) ; 18.7\left(\mathrm{CH}_{3}\right) ; 11.4(\mathrm{CH})$.

IR(neat): 2942 (s); 2866 (s); 2148 (w); 1927 (w); 1732 (s); 1627 (w); 1463 (m); 1367 (m); $1244(\mathrm{~m}) ; 1150(\mathrm{~s}) ; 1102(\mathrm{~m}) ; 996(\mathrm{~m}) ; 882(\mathrm{~m}) ; 673(\mathrm{~s}) \mathrm{cm}^{-1}$.

MS (ESI): $m / z(\%): 279.18$ (100), 311.20 (24), 384.29 (23), $367.26(10)[M+H]^{+}, 219.12(6)$, 172.11 (3).

HRMS (ESI) $m / z:[M+H]^{+}$Calcd for $\mathrm{C}_{21} \mathrm{H}_{39} \mathrm{O}_{3} \mathrm{Si}: 367.2668$; found: 367.2668.

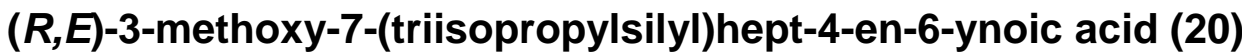

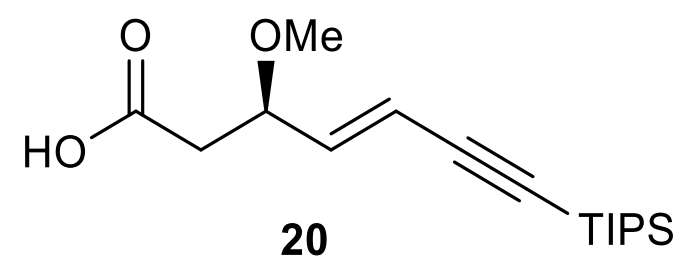

A solution of $E-19(3.2 \mathrm{~g}, 8.74 \mathrm{mmol})$ in formic acid $(13 \mathrm{~mL})$ was stirred overnight at room temperature. The mixture was then concentrated in vacuo and azeotropically dried with toluene for 3 times to remove formic acid. Carboxylic acid 20 was obtained $(2.68 \mathrm{~g}, 8.65$ $\mathrm{mmol}, 99 \%$ ) as a slightly yellow liquid.

General Data: $\mathrm{C}_{17} \mathrm{H}_{30} \mathrm{O}_{3} \mathrm{Si}$; FW: 310.20; TLC: $\mathrm{R}_{\mathrm{f}}=0.3$ (pentane/Et $2 \mathrm{O}$ 5:1); UV (+); Vanillin: brown; $[\alpha]_{D}^{20}=+10.22\left(c=0.45, \mathrm{CHCl}_{3}\right)$.

${ }^{1} \mathrm{H}-\mathrm{NMR}\left(600 \mathrm{MHz}, \mathrm{CDCl}_{3}\right): \delta(\mathrm{ppm}): 6.02(\mathrm{dd}, J=16.0,7.4 \mathrm{~Hz}, 1 \mathrm{H}, \mathrm{CH}=\mathrm{CH}) ; 5.81$ (dd, $J=$ 16.0, $1.1 \mathrm{~Hz}, 1 \mathrm{H}, \mathrm{CH}=\mathrm{CH}) ; 4.11-4.04\left(\mathrm{~m}, 1 \mathrm{H}, \mathrm{CHOCH}_{3}\right) ; 3.33\left(\mathrm{~s}, 3 \mathrm{H}, \mathrm{OCH}_{3}\right) ; 2.62(\mathrm{dd}, \mathrm{J}=$ 15.8, $8.5 \mathrm{~Hz}, 1 \mathrm{H}, 1 \mathrm{CH}_{2} \mathrm{CH}$ ); 2.54 (dd, $\left.J=15.8,4.7 \mathrm{~Hz}, 1 \mathrm{H}, 1 \mathrm{CH}_{2} \mathrm{CH}\right) ; 1.07(\mathrm{~s}, 21 \mathrm{H}$, $\left.\mathrm{Si}\left(\mathrm{CH}\left(\mathrm{CH}_{3}\right)\right)_{3}\right)$.

${ }^{13} \mathrm{C}-N M R\left(151 \mathrm{MHz}, \mathrm{CDCl}_{3}\right)$ : $\delta(\mathrm{ppm}): 174.7(\mathrm{C}=\mathrm{O}) ; 141.4(\mathrm{CH}) ; 113.8(\mathrm{CH}) ; 104.1(\mathrm{C}) ; 92.9$ (C); $77.9(\mathrm{CH}) ; 57.2\left(\mathrm{CH}_{3}\right) ; 40.4\left(\mathrm{CH}_{2}\right) ; 18.8\left(\mathrm{CH}_{3}\right) ; 11.4(\mathrm{CH})$.

IR(neat): 2921 (s); 2865 (s); 2142 (w); $1714(\mathrm{~s}) ; 1641(\mathrm{~m}) ; 1563$ (w); 1462 (m); 1377 (w); $1240(\mathrm{~m}) ; 1100(\mathrm{~s}) ; 996(\mathrm{~m}) ; 958(\mathrm{~m}) ; 882(\mathrm{~m}) ; 674(\mathrm{~s}) \mathrm{cm}^{-1}$.

MS (ESI): $m / z(\%): 328.23(100)\left[M_{+} \mathrm{NH}_{4}\right]^{+}, 279.17(43), 638.42(23), 311.20(19)[\mathrm{M}+\mathrm{H}]^{+}$.

HRMS (ESI) $\mathrm{m} / \mathrm{z}$ : $[M+\mathrm{H}]^{+}$Calcd for $\mathrm{C}_{17} \mathrm{H}_{31} \mathrm{O}_{3} \mathrm{Si}: 311.2042$; found: 311.2043 .

\section{methyl ((R,E)-3-methoxy-7-(triisopropylsilyl)hept-4-en-6-ynoyl)-L-serinate (21)}

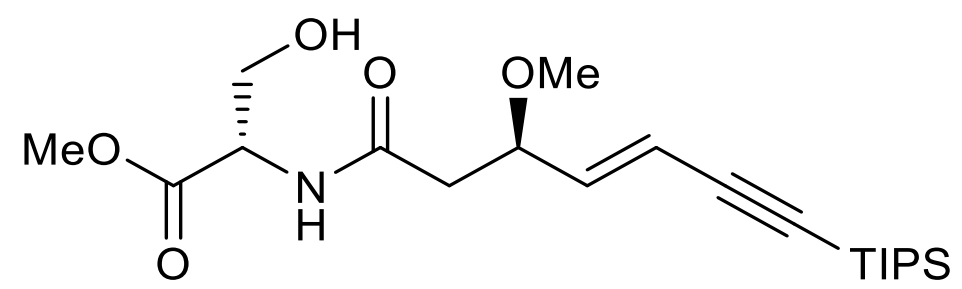

21

DIPEA (3.5 mL, $20.1 \mathrm{mmol}, 2.3 \mathrm{eq})$ and TFFH (2.54 g, $9.61 \mathrm{mmol}, 1.1 \mathrm{eq})$ were added to a solution of carboxylic acid 20 (2.7 g, $8.74 \mathrm{mmol}, 1 \mathrm{eq})$ in THF (30 mL) and the mixture was stirred for $2 \mathrm{~h}$ at room temperature. L-serine methyl ester hydrochloride $(1.63 \mathrm{~g}, 10.49 \mathrm{mmol}$, $1.2 \mathrm{eq})$ was added and the mixture was stirred for $3 \mathrm{~h}$. Et $2 \mathrm{O}(20 \mathrm{~mL})$ was added and the solution 
was washed with $1 \mathrm{M} \mathrm{HCl}(40 \mathrm{~mL})$. The aqueous phase was extracted with $\mathrm{Et}_{2} \mathrm{O}(3 \times 40 \mathrm{~mL})$ and the organic layers were dried over $\mathrm{Na}_{2} \mathrm{SO}_{4}$, filtered and concentrated in vacuo. Purification of the residue by flash chromatography $\left(\mathrm{Et}_{2} \mathrm{O}\right)$ afforded the serinate 21 (3.22 g, $7.82 \mathrm{mmol}, 90 \%$ ) as a yellow oil.

General Data: $\mathrm{C}_{21} \mathrm{H}_{37} \mathrm{NO}_{5} \mathrm{Si}$; FW: 411.24; TLC: $\mathrm{R}_{\mathrm{f}}=0.25$ (Et2O); UV (+); Vanillin: brown; $[\alpha]_{D}^{20}=+28.42\left(c=1.2, \mathrm{CHCl}_{3}\right)$.

1H-NMR (400 MHz, CD $3 \mathrm{OD}): \delta(p p m): 6.05$ (dd, J=16.0, $7.2 \mathrm{~Hz}, 1 \mathrm{H}, \mathrm{CH}=\mathrm{CH}$ ); 5.82 (dd, J $=16.0,1.0 \mathrm{~Hz}, 1 \mathrm{H}, \mathrm{CH}=\mathrm{CH}) ; 4.51(\mathrm{t}, J=4.4 \mathrm{~Hz}, 1 \mathrm{H}, \mathrm{COCHNH}) ; 4.13-4.04\left(\mathrm{~m}, 1 \mathrm{H}, \mathrm{CHOCH}_{3}\right)$; $3.87\left(\mathrm{dd}, J=11.2,4.6 \mathrm{~Hz}, 1 \mathrm{H}, \mathrm{CH}_{2} \mathrm{OH}\right.$ ); $3.76\left(\mathrm{dd}, J=11.2,4.2 \mathrm{~Hz}, 1 \mathrm{H}, \mathrm{CH}_{2} \mathrm{OH}\right.$ ); 3.74 (s, $\left.3 \mathrm{H}, \mathrm{COOCH}_{3}\right) ; 3.30$ (s, 3H, $\left.\mathrm{CHOCH}_{3}\right) ; 2.56$ (dd, $J=14.5,8.1 \mathrm{~Hz}, 1 \mathrm{H}, 1 \mathrm{CH}_{2} \mathrm{CH}$ ); 2.41 (dd, $J$ $\left.=14.5,5.0 \mathrm{~Hz}, 1 \mathrm{H}, 1 \mathrm{CH}_{2} \mathrm{CH}\right) ; 1.08\left(\mathrm{~s}, 21 \mathrm{H}, \mathrm{Si}\left(\mathrm{CH}\left(\mathrm{CH}_{3}\right)\right) 3\right)$.

${ }^{13} \mathrm{C}-N M R\left(100 \mathrm{MHz}, \mathrm{CD}_{3} \mathrm{OD}\right)$ : $\delta(\mathrm{ppm}): 172.7(\mathrm{C}=\mathrm{O}) ; 172.2(\mathrm{C}=\mathrm{O}) ; 144.0(\mathrm{CH}) ; 113.5(\mathrm{CH})$; $106.0(\mathrm{C}) ; 92.4(\mathrm{C}) ; 79.6(\mathrm{CH}) ; 62.9\left(\mathrm{CH}_{2}\right) ; 57.3(\mathrm{CH}) ; 56.1\left(\mathrm{CH}_{3}\right) ; 52.8\left(\mathrm{CH}_{3}\right) ; 42.7\left(\mathrm{CH}_{2}\right)$; $19.0\left(\mathrm{CH}_{3}\right) ; 12.5(\mathrm{CH})$.

IR(neat): 3343 (br); 2943 (m); 2865 (m); 2129 (w); 2048 (w); 1745 (m); 1650 (m); 1538 (m); $1462(\mathrm{~m}) ; 1364(\mathrm{w}) ; 1213(\mathrm{~m}) ; 1074(\mathrm{~m}) ; 959(\mathrm{w}) ; 882(\mathrm{~m}) ; 675(\mathrm{~m}) \mathrm{cm}^{-1}$.

MS (El, $70 \mathrm{eV}): m / z(\%): 411.24(100)\left[M^{+}, 396.22\right.$ (67), $412.25(28), 397.22(22), 393.24$ (17).

HRMS (El, 70eV) m/z: [M]+ Calcd for $\mathrm{C}_{21} \mathrm{H}_{37} \mathrm{NO}_{5} \mathrm{Si}: 411.2436$; found: 411.2442 .

\section{methyl 2-((R,E)-2-methoxy-6-(triisopropylsilyl)hex-3-en-5-yn-1-yl)-4,5- dihydrooxazole-4-carboxylate (22)}

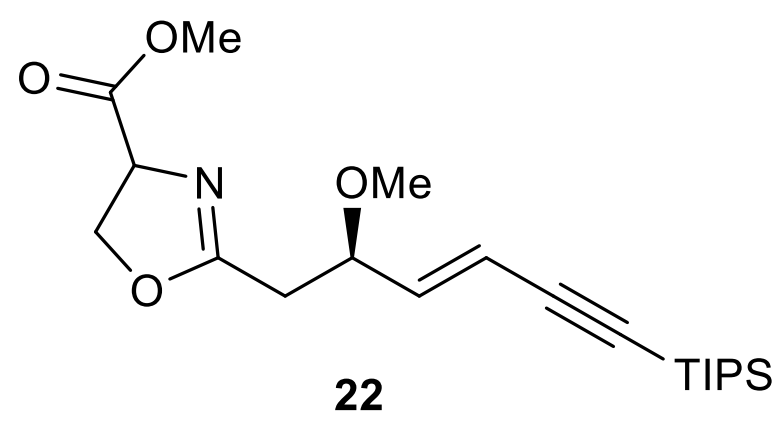

DAST (1.13 mL, $8.56 \mathrm{mmol}, 1.1 \mathrm{eq})$ was added dropwise to a solution of serinate 21 (3.2 g, $7.78 \mathrm{mmol}, 1 \mathrm{eq})$ in $\mathrm{CH}_{2} \mathrm{Cl}_{2}(60 \mathrm{~mL})$ at $-78^{\circ} \mathrm{C}$ and the mixture was stirred for $2 \mathrm{~h}$ at $-78^{\circ} \mathrm{C}$. $\mathrm{K}_{2} \mathrm{CO}_{3}(2.15 \mathrm{~g}, 15.56 \mathrm{mmol}, 2 \mathrm{eq})$ was added and the mixture was warmed to room temperature and stirred for $1 \mathrm{~h}$. A saturated aqueous solution of $\mathrm{NH}_{4} \mathrm{Cl}(50 \mathrm{~mL})$ was carefully added and, after the gas evolution ceased, the layers were separated. The aqueous phase was extracted with $\mathrm{CH}_{2} \mathrm{Cl}_{2}(3 \times 50 \mathrm{~mL})$ and the combined organic extracts were washed with Brine, dried over $\mathrm{Na}_{2} \mathrm{SO}_{4}$, filtered and concentrated in vacuo to afford the crude product 22 as a yellow oil, which was used for the next step without further purification.

General Data: $\mathrm{C}_{21} \mathrm{H}_{35} \mathrm{NO}_{4} \mathrm{Si}$; FW: 393.23; TLC: $\mathrm{R}_{\mathfrak{f}}=0.5$ (Et2O); UV (+); Vanillin: brown; $[\alpha]_{D}^{20}$ $=+58.59\left(c=0.85, \mathrm{CHCl}_{3}\right)$.

1H-NMR (400 MHz, CDCl $): \delta(p p m): 6.03(\mathrm{dd}, J=16.0,7.5 \mathrm{~Hz}, 1 \mathrm{H}, \mathrm{CH}=\mathrm{CH}$ ); 5.77 (dd, $J=$ 16.0, $1.0 \mathrm{~Hz}, 1 \mathrm{H}, \mathrm{CH}=\mathrm{CH}$ ); $4.74\left(\mathrm{dd}, J=10.5,7.7 \mathrm{~Hz}, 1 \mathrm{H}, \mathrm{OCH}_{2} \mathrm{CHCO}\right.$ ); 4.50 (dd, $J=8.8$, $7.8 \mathrm{~Hz}, 1 \mathrm{H}, 1 \mathrm{OCH} \mathrm{H}_{2} \mathrm{CHCO}$ ); 4.38 (dd, $J=10.5,8.8 \mathrm{~Hz}, 1 \mathrm{H}, 1 \mathrm{OCH} \mathrm{H}_{2} \mathrm{CHCO}$ ); 4.07-3.99 (m, $1 \mathrm{H}$, $\left.\mathrm{CHOCH}_{3}\right) ; 3.77\left(\mathrm{~s}, 3 \mathrm{H}, \mathrm{COOCH}_{3}\right) ; 3.29\left(\mathrm{~s}, 3 \mathrm{H}, \mathrm{CHOCH}_{3}\right) ; 2.66$ (dd, J = 14.9, $7.7 \mathrm{~Hz}, 1 \mathrm{H}$, $\left.1 \mathrm{CH}_{2} \mathrm{CH}\right) ; 2.49$ (dd, $\left.J=14.9,5.8 \mathrm{~Hz}, 1 \mathrm{H}, 1 \mathrm{CH}_{2} \mathrm{CH}\right) ; 1.06\left(\mathrm{~s}, 21 \mathrm{H}, \mathrm{Si}\left(\mathrm{CH}\left(\mathrm{CH}_{3}\right)\right)_{3}\right)$.

${ }^{13} \mathrm{C}-N M R\left(100 \mathrm{MHz}, \mathrm{CDCl}_{3}\right): \delta(\mathrm{ppm}): 171.6(\mathrm{C}=\mathrm{N}) ; 167.5(\mathrm{C}=\mathrm{O}) ; 142.1(\mathrm{CH}) ; 113.3(\mathrm{CH})$; $104.4(\mathrm{C}) ; 92.4(\mathrm{C}) ; 78.5(\mathrm{CH}) ; 69.5(\mathrm{CH}) ; 68.2\left(\mathrm{CH}_{2}\right) ; 57.1\left(\mathrm{CH}_{3}\right) ; 52.8\left(\mathrm{CH}_{3}\right) ; 34.4\left(\mathrm{CH}_{2}\right)$; $18.7\left(\mathrm{CH}_{3}\right) ; 11.4(\mathrm{CH})$. 
IR(neat): 3738 (w); 3649 (w); 2980 (s); 2890 (m); 2866 (m); 2328 (w); 2130 (w); 1743 (m); $1628(\mathrm{w}) ; 1462(\mathrm{~m}) ; 1384(\mathrm{~m}) ; 1250(\mathrm{~m}) ; 1156(\mathrm{~m}) ; 1073(\mathrm{~m}) ; 994(\mathrm{~m}) ; 956(\mathrm{~m}) ; 882(\mathrm{~m})$; $664(\mathrm{~m}) \mathrm{cm}^{-1}$.

MS (El, 70eV): m/ $z$ (\%): 350.16 (100), 337.22 (96), 361.18 (87), 393.23 (45) [M], 368.17 (43), 362.19 (36), 351.17 (35).

HRMS (El, 70eV) m/z: [M] ${ }^{+}$Calcd for $\mathrm{C}_{21} \mathrm{H}_{35} \mathrm{NO}_{4} \mathrm{Si}: 393.2330$; found: 393.2334.

\section{methyl (R,E)-2-(2-methoxy-6-(triisopropylsilyl)hex-3-en-5-yn-1-yl)oxazole-4- carboxylate (23)}

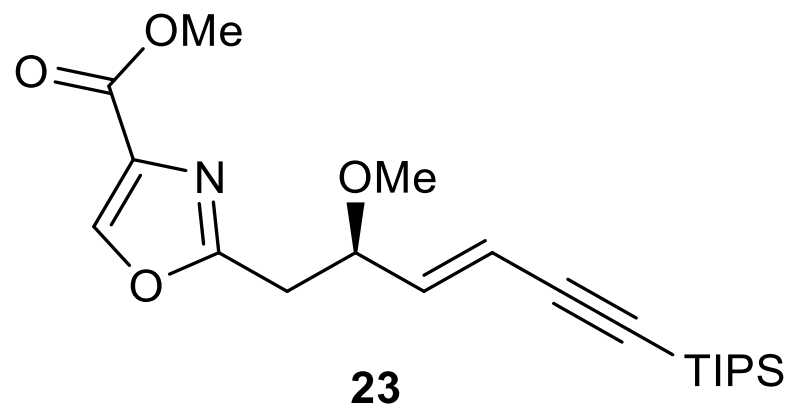

The crude material 22 was dissolved in $\mathrm{CH}_{2} \mathrm{Cl}_{2}(60 \mathrm{~mL})$, cooled to $0^{\circ} \mathrm{C}$ and protected from light with aluminium foil. DBU (2.24 mL, $15.56 \mathrm{mmol}, 2 \mathrm{eq})$ and $\mathrm{BrCCl}_{3}(1.53 \mathrm{~mL}, 15.56$ $\mathrm{mmol}, 2$ eq) were sequentially added dropwise, then the bath was removed and the mixture was stirred at room temperature for $16 \mathrm{~h}$. The reaction was quenched with saturated aqueous $\mathrm{NH}_{4} \mathrm{Cl}$ solution $(100 \mathrm{~mL})$ and the layers were separated. The aqueous phase was extracted with $\mathrm{CH}_{2} \mathrm{Cl}_{2}(3 \times 100 \mathrm{~mL})$ and the organic layers were dried over $\mathrm{Na}_{2} \mathrm{SO}_{4}$, filtered and concentrated in vacuo. Purification of the residue by flash chromatography (pentane/ $\mathrm{Et}_{2} \mathrm{O} 2: 1$ ) afforded oxazole $23(1.77 \mathrm{~g}, 4.51 \mathrm{mmol}, 62 \%$ from 21$)$ as a yellow oil.

General Data: $\mathrm{C}_{21} \mathrm{H}_{33} \mathrm{NO}_{4} \mathrm{Si}$; FW: 391.22; TLC: $\mathrm{Rf}=0.35$ (pentane/Et $2 \mathrm{O}$ 2:1); UV (+); Vanillin: brown; $[\alpha]_{D}^{20}=-17.4\left(c=0.5, \mathrm{CHCl}_{3}\right)$.

${ }^{1} \mathrm{H}-\mathrm{NMR}\left(400 \mathrm{MHz}, \mathrm{CDCl}_{3}\right): \delta(\mathrm{ppm}): 8.16(\mathrm{~s}, 1 \mathrm{H}, \mathrm{NC}=\mathrm{CH}) ; 6.04(\mathrm{dd}, J=15.8,7.4 \mathrm{~Hz}, 1 \mathrm{H}$, $\mathrm{CH}=\mathrm{CH}) ; 5.77(\mathrm{dd}, \mathrm{J}=15.9,1.0 \mathrm{~Hz}, 1 \mathrm{H}, \mathrm{CH}=\mathrm{CH}) ; 4.18-4.11(\mathrm{~m}, 1 \mathrm{H}, \mathrm{CHOCH} 3) ; 3.90(\mathrm{~s}, 3 \mathrm{H}$, $\left.\mathrm{COOCH}_{3}\right) ; 3.26\left(\mathrm{~s}, 3 \mathrm{H}, \mathrm{CHOCH}_{3}\right) ; 3.08\left(\mathrm{dd}, J=15.1,8.1 \mathrm{~Hz}, 1 \mathrm{H}, 1 \mathrm{CH}_{2} \mathrm{CH}\right.$ ); 2.99 (dd, $J=$ $\left.15.1,5.3 \mathrm{~Hz}, 1 \mathrm{H}, 1 \mathrm{CH}_{2} \mathrm{CH}\right) ; 1.07\left(\mathrm{~s}, 21 \mathrm{H}, \mathrm{Si}\left(\mathrm{CH}\left(\mathrm{CH}_{3}\right)\right)_{3}\right)$.

${ }^{13} \mathrm{C}$-NMR (100 MHz, CDCl 3$)$ : $\delta(\mathrm{ppm}): 162.6(\mathrm{C}=\mathrm{O}) ; 161.8(\mathrm{C}=\mathrm{N}) ; 144.1(\mathrm{CH}) ; 141.7(\mathrm{CH})$; $133.5(\mathrm{C}) ; 113.8(\mathrm{CH}) ; 104.1(\mathrm{C}) ; 92.9(\mathrm{C}) ; 79.3(\mathrm{CH}) ; 57.1\left(\mathrm{CH}_{3}\right) ; 52.3\left(\mathrm{CH}_{3}\right) ; 34.6\left(\mathrm{CH}_{2}\right)$; $18.7\left(\mathrm{CH}_{3}\right) ; 11.4(\mathrm{CH})$.

IR(neat): 3867 (w); 3738 (w); 3649 (w); 2980 (s); 2866 (m); 2328 (w); 2137 (w); 1747 (m); $1584(\mathrm{~m}) ; 1462(\mathrm{~m}) ; 1384(\mathrm{~m}) ; 1323(\mathrm{~m}) ; 1239(\mathrm{~m}) ; 1165(\mathrm{~m}) ; 1107(\mathrm{~m}) ; 997(\mathrm{~m}) ; 956(\mathrm{~m})$; $882(\mathrm{~m}) ; 763(\mathrm{~m}) ; 664(\mathrm{~m}) \mathrm{cm}^{-1}$.

MS (ESI): $m / z(\%): 360.20(100), 392.22(65)[M+\mathrm{H}]^{+}, 414.20(20)[M+N a]^{+}, 805.42(4)$ HRMS (ESI) $\mathrm{m} / \mathrm{z}:[\mathrm{M}+\mathrm{H}]^{+}$Calcd for $\mathrm{C}_{21} \mathrm{H}_{34} \mathrm{NO}_{4} \mathrm{Si}: 392.2257$; found: 392.2259 . 


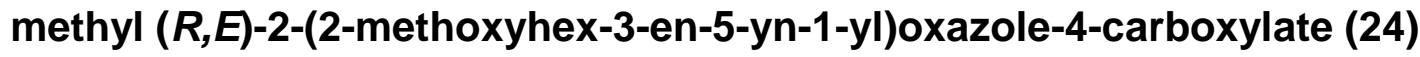

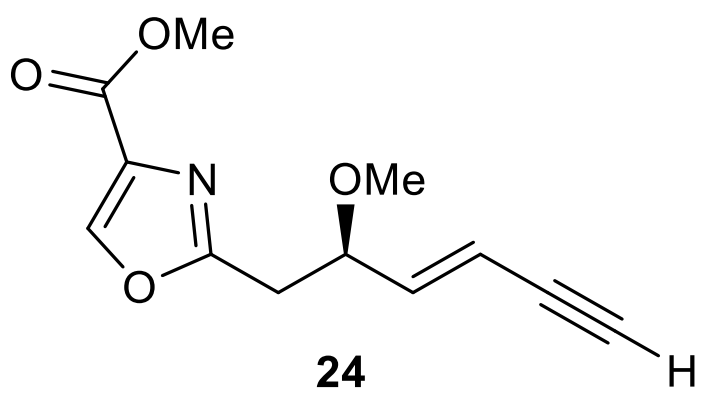

TBAF ( $1 \mathrm{M}$ in THF, $4.21 \mathrm{~mL}, 4.21 \mathrm{mmol}, 1.1 \mathrm{eq})$ was added dropwise at $0^{\circ} \mathrm{C}$ to a solution of TIPS oxazole $23(1.5 \mathrm{~g}, 3.83 \mathrm{mmol}, 1 \mathrm{eq})$ in THF $(40 \mathrm{~mL})$. The mixture was stirred for 30 $\mathrm{min}$ at room temperature and then quenched with water $(30 \mathrm{~mL})$. The aqueous phase was extracted with $\mathrm{Et}_{2} \mathrm{O}(3 \times 20 \mathrm{~mL})$ and the organic layers were dried over $\mathrm{Na}_{2} \mathrm{SO}_{4}$, filtered and concentrated in vacuo. Purification of the residue by flash chromatography (pentane/Et $2 \mathrm{O}$ 2:1 to $1: 1)$ afforded oxazole $24(729 \mathrm{mg}, 3.1 \mathrm{mmol}, 81 \%)$ as a yellow oil.

General Data: $\mathrm{C}_{12} \mathrm{H}_{13} \mathrm{NO}_{4}$; FW: 235.08; TLC: $\mathrm{Rf}=0.30$ (pentane/Et $\mathrm{t}_{2} \mathrm{O}$ 1:1); UV (+); Vanillin: brown; $[\alpha]_{D}^{20}=-26.89\left(c=0.45, \mathrm{CHCl}_{3}\right)$.

${ }^{1} \mathrm{H}-\mathrm{NMR}\left(400 \mathrm{MHz}, \mathrm{CDCl}_{3}\right): \delta(\mathrm{ppm}): 8.16(\mathrm{~s}, 1 \mathrm{H}, \mathrm{NC}=\mathrm{CH}) ; 6.10$ (ddd, $J=16.0,7.4,0.5 \mathrm{~Hz}$, $1 \mathrm{H}, \mathrm{CH}=\mathrm{CH}) ; 5.70$ (ddd, $J=16.0,2.3,1.0 \mathrm{~Hz}, 1 \mathrm{H}, \mathrm{CH}=\mathrm{CH}) ; 4.19-4.12\left(\mathrm{~m}, 1 \mathrm{H}, \mathrm{CHOCH}_{3}\right)$; $3.89\left(\mathrm{~s}, 3 \mathrm{H}, \mathrm{COOCH}_{3}\right) ; 3.26\left(\mathrm{~s}, 3 \mathrm{H}, \mathrm{CHOCH}_{3}\right) ; 3.08\left(\mathrm{dd}, \mathrm{J}=15.1,7.8 \mathrm{~Hz}, 1 \mathrm{H}, 1 \mathrm{CH}_{2} \mathrm{CH}\right)$; $2.98\left(\mathrm{dd}, J=15.0,5.8 \mathrm{~Hz}, 1 \mathrm{H}, 1 \mathrm{CH}_{2} \mathrm{CH}\right) ; 2.92(\mathrm{~d}, J=2.3 \mathrm{~Hz}, 1 \mathrm{H}, \mathrm{CCH})$.

${ }^{13} \mathrm{C}$-NMR $\left(100 \mathrm{MHz}, \mathrm{CDCl}_{3}\right)$ : $\delta(\mathrm{ppm}): 162.4(\mathrm{C}=\mathrm{O}) ; 161.7(\mathrm{C}=\mathrm{N}) ; 144.1(\mathrm{CH}) ; 143.1(\mathrm{CH})$; $133.5(\mathrm{C}) ; 112.4(\mathrm{CH}) ; 80.9(\mathrm{C}) ; 79.1(\mathrm{CH}) ; 79.0(\mathrm{CH}) ; 57.1\left(\mathrm{CH}_{3}\right) ; 52.3\left(\mathrm{CH}_{3}\right) ; 34.5\left(\mathrm{CH}_{2}\right)$.

IR(neat): 3251 (m); 3119 (w); 2941 (w); 2882 (w); 2826 (w); 1712 (s); 1582 (s); 1437 (m); 1323 (s); $1231(\mathrm{~m}) ; 1200(\mathrm{~m}) ; 1163(\mathrm{~m}) ; 1095(\mathrm{~s}) ; 1006(\mathrm{~m}) ; 970(\mathrm{~m}) ; 940(\mathrm{~m}) ; 810(\mathrm{~m}) ; 768$ (m); $679(\mathrm{~m}) \mathrm{cm}^{-1}$.

MS (ESI): $m / z(\%): 204.06$ (100), $236.09(97)[M+H]^{+}, 156.12$ (55), 486.19 (32), 469.16 (28), 279.18 (22), 311.03 (19).

HRMS (ESI) $\mathrm{m} / \mathrm{z}:[\mathrm{M}+\mathrm{H}]^{+}$Calcd for $\mathrm{C}_{12} \mathrm{H}_{14} \mathrm{NO}_{4}$ : 236.0923; found: 236.0935 .

methyl 2-((2R,3E,7Z,10S,12S,13E)-2-methoxy-12-(methoxymethoxy)-11,11-dimethyl10-((triethylsilyl)oxy)pentadeca-3,7,13-trien-5-yn-1-yl)oxazole-4-carboxylate (25)

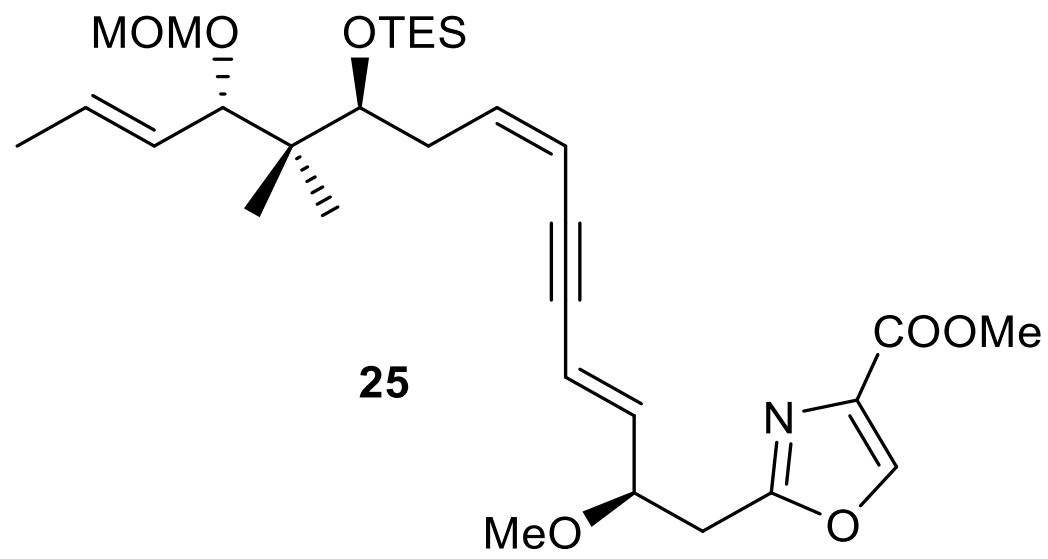

The vinyl iodide 14 (397 $\mathrm{mg}, 0.847 \mathrm{mmol}, 1$ eq) was dissolved in degassed $\mathrm{CH}_{3} \mathrm{CN}(5 \mathrm{~mL})$ and Cul (39 mg, $0.254 \mathrm{mmol}, 0.3 \mathrm{eq})$ and $\mathrm{PdCl}_{2}\left(\mathrm{PPh}_{3}\right)_{2}(60 \mathrm{mg}, 0.0847 \mathrm{mmol}, 0.1 \mathrm{eq})$ were 
added. The mixture was degassed by freeze-pump-thaw ( 2 cycles) and then cooled to $-15^{\circ} \mathrm{C}$ (ice/acetone bath). NEt3 $(0.706 \mathrm{~mL}, 5.08 \mathrm{mmol}, 6 \mathrm{eq})$ was added, followed by a slow addition of the enyne 24 (240 mg, $1.02 \mathrm{mmol}, 1.2 \mathrm{eq})$ in degassed $\mathrm{CH}_{3} \mathrm{CN}$ (3 mL). The solution became red and after $15 \mathrm{~min}$ the bath was removed. The mixture was stirred for $1 \mathrm{~h}$ at room temperature and quenched with saturated aqueous $\mathrm{NH}_{4} \mathrm{Cl}$ solution $(10 \mathrm{~mL})$. The aqueous phase was extracted with $\mathrm{Et}_{2} \mathrm{O}(3 \times 10 \mathrm{~mL})$ and the combined organic extracts were dried over $\mathrm{Na}_{2} \mathrm{SO}_{4}$, filtered and concentrated in vacuo. The residue was purified by flash chromatography (pentane/Et2O 2:1) to give the monomer 25 (414 mg, $0.720 \mathrm{mmol}, 85 \%)$ as a yellow oil.

General Data: $\mathrm{C}_{31} \mathrm{H}_{49} \mathrm{NO}_{7} \mathrm{Si}$; FW: 575.33; TLC: $\mathrm{R}_{\mathrm{f}}=0.20$ (pentane/Et ${ }_{2} \mathrm{O}$ 2:1); UV (+); Vanillin: black; $[\alpha]_{D}^{20}=-28.4\left(c=0.5, \mathrm{CHCl}_{3}\right)$.

${ }^{1} \mathrm{H}-\mathrm{NMR}\left(600 \mathrm{MHz}, \mathrm{CDCl}_{3}\right): \delta(\mathrm{ppm}): 8.16(\mathrm{~s}, 1 \mathrm{H}, \mathrm{NC}=\mathrm{CH}) ; 6.10-6.02\left(\mathrm{~m}, 1 \mathrm{H}, \mathrm{CH}_{2} \mathrm{CH}=\mathrm{CH}\right)$; 5.99 (dd, $J=15.9,7.5 \mathrm{~Hz}, 1 \mathrm{H}, \mathrm{CCH}=\mathrm{CHCH}$ ); 5.89 (dd, $J=15.9,2.2 \mathrm{~Hz}, 1 \mathrm{H}, \mathrm{CCH}=\mathrm{CHCH}_{2}$ ); 5.66-5.54 (m, 2H, CH $\left.\left(\mathrm{OCH}_{3}\right) \mathrm{CH}=\mathrm{CH}, \mathrm{CH}=\mathrm{CHCH}_{3}\right) ; 5.37-5.28\left(\mathrm{~m}, 1 \mathrm{H}, \mathrm{CH}=\mathrm{CHCH}_{3}\right) ; 4.66(\mathrm{~d}$, $\left.J=6.6 \mathrm{~Hz}, 1 \mathrm{H}, 1 \mathrm{OCH}_{2} \mathrm{OCH}_{3}\right) ; 4.46\left(\mathrm{~d}, J=6.6 \mathrm{~Hz}, 1 \mathrm{H}, 1 \mathrm{OCH}_{2} \mathrm{OCH}_{3}\right) ; 4.21-4.13(\mathrm{~m}, 1 \mathrm{H}$, $\mathrm{CHOCH}_{3}$ ); 3.90 (s, $3 \mathrm{H}, \mathrm{COOCH}_{3}$ ); $3.84\left(\mathrm{~d}, J=9.3 \mathrm{~Hz}, 1 \mathrm{H}, \mathrm{CH}_{2} \mathrm{CHOTES}\right.$ ); 3.67 (dd, $J=7.1$, $3.8 \mathrm{~Hz}, 1 \mathrm{H}, \mathrm{CH}_{2} \mathrm{CHOTES}$ ); 3.35 (s, 3H, $\mathrm{CHOCH}_{3}$ ); 3.27 (s, 3H, $\mathrm{CHOCH}_{2} \mathrm{OCH}_{3}$ ); 3.10 (dd, J $\left.=12.6,5.6 \mathrm{~Hz}, 1 \mathrm{H}, 1 \mathrm{CH}_{2} \mathrm{CHOCH}_{3}\right) ; 2.99$ (dd, $J=15.0,5.5 \mathrm{~Hz}, 1 \mathrm{H}, 1 \mathrm{CH}_{2} \mathrm{CHOCH}_{3}$ ); 2.62$2.53\left(\mathrm{~m}, 1 \mathrm{H}, 1 \mathrm{CH}_{2} \mathrm{CH}=\mathrm{CH}\right) ; 2.45-2.37\left(\mathrm{~m}, 1 \mathrm{H}, 1 \mathrm{CH}_{2} \mathrm{CH}=\mathrm{CH}\right) ; 1.71$ (dd, $J=6.4,1.6 \mathrm{~Hz}, 3 \mathrm{H}$, $\left.\mathrm{CH}=\mathrm{CHCH}_{3}\right) ; 0.952$ (app t, $\left.J=8.0 \mathrm{~Hz}, 9 \mathrm{H}, \mathrm{OSi}\left(\mathrm{CH}_{2} \mathrm{CH}_{3}\right) 3\right) ; 0.922$ (s, 3H, CCH ); 0.849 (s, $\left.3 \mathrm{H}, \mathrm{CCH}_{3}\right) ; 0.599\left(\mathrm{q}, J=8.0 \mathrm{~Hz}, 6 \mathrm{H}, \mathrm{OSi}\left(\mathrm{CH}_{2} \mathrm{CH}_{3}\right)_{3}\right)$.

${ }^{13} \mathrm{C}-N M R\left(151 \mathrm{MHz}, \mathrm{CDCl}_{3}\right): \delta(\mathrm{ppm}): 162.6(\mathrm{C}=\mathrm{O}) ; 161.8(\mathrm{C}=\mathrm{N}) ; 144.1(\mathrm{CH}) ; 143.2(\mathrm{CH})$; $140.4(\mathrm{CH}) ; 133.5(\mathrm{C}) ; 131.0(\mathrm{CH}) ; 128.0(\mathrm{CH}) ; 113.9(\mathrm{CH}) ; 109.5(\mathrm{CH}) ; 93.6\left(\mathrm{CH}_{2}\right) ; 91.2$ (C); $88.5(\mathrm{C}) ; 81.6(\mathrm{CH}) ; 79.4(\mathrm{CH}) ; 76.5(\mathrm{CH}) ; 56.9\left(\mathrm{CH}_{3}\right) ; 55.7\left(\mathrm{CH}_{3}\right) ; 52.3\left(\mathrm{CH}_{3}\right) ; 43.1(\mathrm{C})$; $34.7\left(\mathrm{CH}_{2}\right) ; 34.5\left(\mathrm{CH}_{2}\right) ; 19.8\left(\mathrm{CH}_{3}\right) ; 19.4\left(\mathrm{CH}_{3}\right) ; 18.0\left(\mathrm{CH}_{3}\right) ; 7.3\left(\mathrm{CH}_{3}\right) ; 5.7\left(\mathrm{CH}_{2}\right)$.

IR(neat): $3656(\mathrm{w}) ; 2980(\mathrm{~s}) ; 2884(\mathrm{~m}) ; 1749(\mathrm{~m}) ; 1585(\mathrm{~m}) ; 1461(\mathrm{~m}) ; 1382(\mathrm{~m}) ; 1238(\mathrm{~m})$; $1142(\mathrm{~m}) ; 1091$ (s); 1036 (s); 1004 (s); 956 (s); 923 (m); $727(\mathrm{~s}) ; 678(\mathrm{~m}) ; 604$ (w) cm-1. MS (ESI): $m / z(\%): 418.20$ (100), 514.29 (81), 598.31 (22), 593.36 (18) $\left[\mathrm{M}_{+} \mathrm{NH}_{4}\right]^{+}, 350.17$ (8), $482.27(6), 576.33(<1)[M+\mathrm{H}]^{+}$.

HRMS (ESI) $\mathrm{m} / \mathrm{z}:[\mathrm{M}+\mathrm{H}]^{+}$Calcd for $\mathrm{C}_{31} \mathrm{H}_{50} \mathrm{NO}$ (Si: 576.3357 ; found: 576.3363 .

\section{methyl 2-((2R,3E,7Z,10S,12S,13E)-10-hydroxy-2-methoxy-12-(methoxymethoxy)-} 11,11-dimethylpentadeca-3,7,13-trien-5-yn-1-yl)oxazole-4-carboxylate (26)

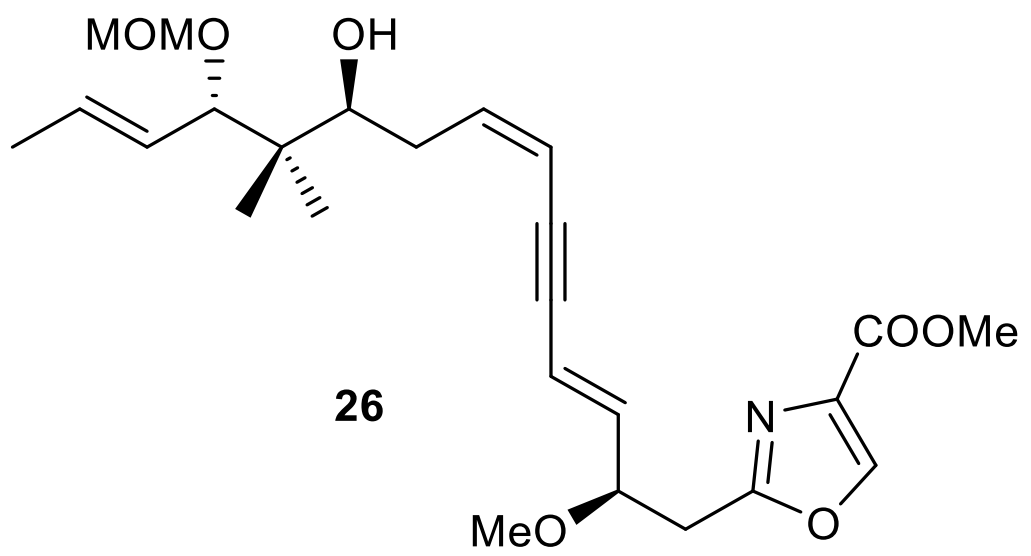

CSA $(10 \mathrm{mg}, 0.0444 \mathrm{mmol}, 0.2 \mathrm{eq})$ was added at $0^{\circ} \mathrm{C}$ to a solution of TES protected monomer 25 (128 mg, $0.222 \mathrm{mmol}, 1 \mathrm{eq})$ in $\mathrm{CH}_{2} \mathrm{Cl}_{2}(6 \mathrm{~mL})$ and $\mathrm{MeOH}(6 \mathrm{~mL})$. The mixture was stirred for $1 \mathrm{~h}$ at $0^{\circ} \mathrm{C}$ under normal atmosphere. Saturated aqueous $\mathrm{NaHCO}_{3}$ solution $(15 \mathrm{~mL})$ was added and the layers were separated. The aqueous phase was extracted with $\mathrm{CH}_{2} \mathrm{Cl}_{2}(3 \times 10 \mathrm{~mL})$ and the combined organic extracts were dried over $\mathrm{Na}_{2} \mathrm{SO}_{4}$, filtered and 
concentrated in vacuo. The residue was purified by flash chromatography $\left(\mathrm{Et}_{2} \mathrm{O} / \mathrm{pentane}\right.$ $2: 1)$ giving deprotected monomer $26(97 \mathrm{mg}, 0.210 \mathrm{mmol}, 95 \%)$ as a slightly yellow oil.

General Data: $\mathrm{C}_{25} \mathrm{H}_{35} \mathrm{NO}_{7}$; $\mathrm{FW}: 461.24$; TLC: $\mathrm{R}_{\mathrm{f}}=0.25$ (Et ${ }_{2} \mathrm{O} /$ Pentane 2:1); UV (+); Vanillin: black; $[\alpha]_{D}^{20}=-36.11\left(c=1.75, \mathrm{CHCl}_{3}\right)$.

${ }^{1} \mathrm{H}-\mathrm{NMR}\left(600 \mathrm{MHz}, \mathrm{CDCl}_{3}\right)$ : $\delta$ (ppm): $8.16(\mathrm{~s}, 1 \mathrm{H}, \mathrm{NC}=\mathrm{CH}) ; 6.24-6.15\left(\mathrm{~m}, 1 \mathrm{H}, \mathrm{CH}_{2} \mathrm{CH}=\mathrm{CH}\right)$; $5.96(\mathrm{dd}, J=15.8,7.5 \mathrm{~Hz}, 1 \mathrm{H}, \mathrm{CCH}=\mathrm{CHCH}) ; 5.87\left(\mathrm{~d}, J=15.9 \mathrm{~Hz}, 1 \mathrm{H}, \mathrm{CCH}=\mathrm{CHCH}_{2}\right) ; 5.71-$ $5.61\left(\mathrm{~m}, 2 \mathrm{H}, \mathrm{CH}\left(\mathrm{OCH}_{3}\right) \mathrm{CH}=\mathrm{CH}, \mathrm{CH}=\mathrm{CHCH}_{3}\right) ; 5.43-5.36\left(\mathrm{~m}, 1 \mathrm{H}, \mathrm{CH}=\mathrm{CHCH}_{3}\right) ; 4.65(\mathrm{~d}, \mathrm{~J}=$ $\left.6.5 \mathrm{~Hz}, 1 \mathrm{H}, 1 \mathrm{OCH}_{2} \mathrm{OCH}_{3}\right) ; 4.47\left(\mathrm{~d}, \mathrm{~J}=6.5 \mathrm{~Hz}, 1 \mathrm{H}, 1 \mathrm{OCH}_{2} \mathrm{OCH}_{3}\right) ; 4.21-4.12(\mathrm{~m}, 1 \mathrm{H}$, $\left.\mathrm{CHOCH}_{3}\right) ; 3.90\left(\mathrm{~s}, 3 \mathrm{H}, \mathrm{COOCH}_{3}\right) ; 3.89\left(1 \mathrm{H}, \mathrm{CH}_{2} \mathrm{CHOH}\right) ; 3.68(\mathrm{~d}, J=10.0 \mathrm{~Hz}, 1 \mathrm{H}$, $\mathrm{CHOCH}_{2} \mathrm{OCH}_{3}$ ); 3.38 (s, 3H, $\mathrm{CHOCH}_{3}$ ); 3.26 (s, 3H, $\mathrm{CHOCH}_{2} \mathrm{OCH}_{3}$ ); 3.09 (dd, J = 15.1, 7.9 $\mathrm{Hz}, 1 \mathrm{H}, 1 \mathrm{CH}_{2} \mathrm{CHOCH}_{3}$ ); 2.99 (dd, $J=14.9,5.3 \mathrm{~Hz}, 1 \mathrm{H}, 1 \mathrm{CH}_{2} \mathrm{CHOCH}_{3}$ ); 2.54 (dd, $J=14.3$, $\left.7.6 \mathrm{~Hz}, 1 \mathrm{H}, 1 \mathrm{CH}_{2} \mathrm{CH}=\mathrm{CH}\right) ; 2.37-2.28\left(\mathrm{~m}, 1 \mathrm{H}, 1 \mathrm{CH}_{2} \mathrm{CH}=\mathrm{CH}\right) ; 1.75(\mathrm{~d}, J=6.3 \mathrm{~Hz}, 3 \mathrm{H}$, $\left.\mathrm{CH}=\mathrm{CHCH}_{3}\right) ; 0.917$ (s, 3H, $\left.\mathrm{CCH}_{3}\right) ; 0.867$ (s, 3H, $\left.\mathrm{CCH}_{3}\right)$.

${ }^{13} \mathrm{C}-N M R\left(151 \mathrm{MHz}, \mathrm{CDCl}_{3}\right)$ : $\delta(\mathrm{ppm}): 162.6(\mathrm{C}=\mathrm{O}) ; 161.8(\mathrm{C}=\mathrm{N}) ; 144.1(\mathrm{CH}) ; 143.3(\mathrm{CH})$; $140.3(\mathrm{CH}) ; 133.5(\mathrm{C}) ; 132.0(\mathrm{CH}) ; 126.9(\mathrm{CH}) ; 114.0(\mathrm{CH}) ; 109.8(\mathrm{CH}) ; 93.8\left(\mathrm{CH}_{2}\right) ; 91.1$ (C); $88.3(\mathrm{C}) ; 84.7(\mathrm{CH}) ; 79.4(\mathrm{CH}) ; 76.1(\mathrm{CH}) ; 56.9\left(\mathrm{CH}_{3}\right) ; 56.2\left(\mathrm{CH}_{3}\right) ; 52.3\left(\mathrm{CH}_{3}\right) ; 41.0(\mathrm{C})$; $34.7\left(\mathrm{CH}_{2}\right) ; 32.8\left(\mathrm{CH}_{2}\right) ; 21.1\left(\mathrm{CH}_{3}\right) ; 17.7\left(\mathrm{CH}_{3}\right) ; 18.0\left(\mathrm{CH}_{3}\right)$.

IR(neat): 3483 (br); 3164 (w); 2934 (m); 2826 (w); 2249 (w); 1734 (m); 1585 (m); 1438 (m); $1322(\mathrm{~m}) ; 1166(\mathrm{~m}) ; 1101(\mathrm{~s}) ; 1030(\mathrm{~s}) ; 927(\mathrm{~m}) ; 917(\mathrm{~m}) ; 731(\mathrm{~s}) \mathrm{cm}^{-1}$.

MS (ESI): $\mathrm{m} / z(\%): 304.11$ (100), 400.21 (48), 479.27 (20) $\left[\mathrm{M}_{+} \mathrm{NH}_{4}\right]^{+}, 272.09$ (19), 430.22 (15), $462.24(12)[M+H]^{+}, 209.13(3)$.

HRMS (ESI) $m / z:[M+H]^{+}$Calcd for $\mathrm{C}_{25} \mathrm{H}_{36} \mathrm{NO}_{7}$ : 462.2492; found: 462.2473 .

\section{2-((2R,3E,7Z,10S,12S,13E)-2-methoxy-12-(methoxymethoxy)-11,11-dimethyl-10-} ((triethylsilyl)oxy)pentadeca-3,7,13-trien-5-yn-1-yl)oxazole-4-carboxylic acid (27)

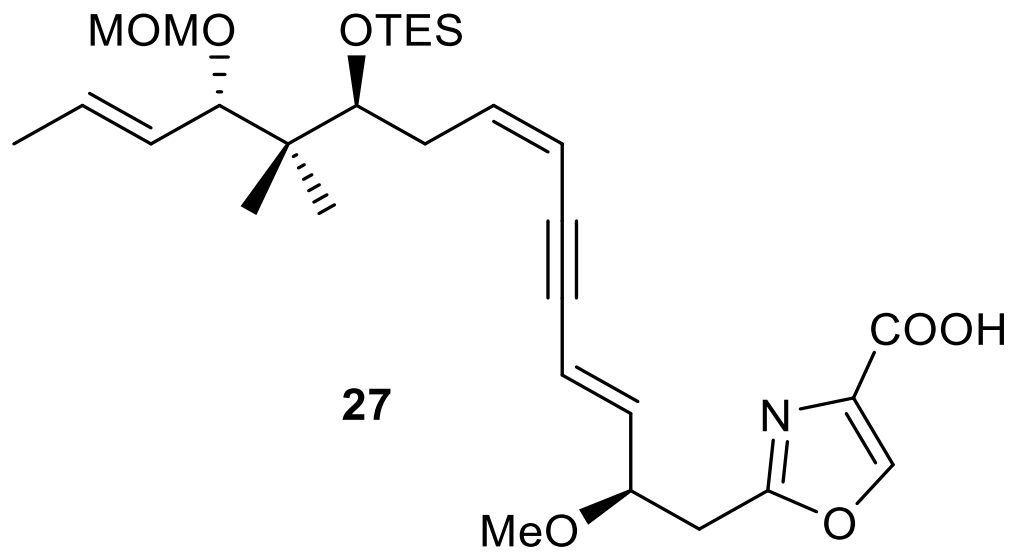

25 (150 mg, $0.261 \mathrm{mmol}, 1 \mathrm{eq})$ was dissolved in THF $(5 \mathrm{~mL})$ and treated at room temperature with $\mathrm{LiOH}\left(1 \mathrm{M}\right.$ in $\left.\mathrm{H}_{2} \mathrm{O}, 0.783 \mathrm{~mL}, 0.783 \mathrm{mmol}, 3 \mathrm{eq}\right)$. The mixture was stirred for $3 \mathrm{~h}$ at room temperature and neutralized with $1 \mathrm{M} \mathrm{HCl}(2 \mathrm{~mL})$. The aqueous phase was extracted with $\mathrm{Et}_{2} \mathrm{O}(3 \times 3 \mathrm{~mL})$ and the organic extracts were dried over $\mathrm{Na}_{2} \mathrm{SO}_{4}$, filtered and concentrated in vacuo to give acid 27 (146 mg, $0.259 \mathrm{mmol}, 99 \%)$ as a yellow oil, which was used for the next step without further purification.

General Data: $\mathrm{C}_{30} \mathrm{H}_{47} \mathrm{NO}_{7} \mathrm{Si}$; FW: 561.31; TLC: UV (+); Vanillin: black; $[\alpha]_{D}^{20}=-36.9(c=1.0$, $\left.\mathrm{CHCl}_{3}\right)$.

${ }^{1} \mathrm{H}-\mathrm{NMR}\left(600 \mathrm{MHz}, \mathrm{CDCl}_{3}\right): \delta(\mathrm{ppm}): 8.24(\mathrm{~s}, 1 \mathrm{H}, \mathrm{NC}=\mathrm{CH}) ; 6.09-6.02\left(\mathrm{~m}, 1 \mathrm{H}, \mathrm{CH}_{2} \mathrm{CH}=\mathrm{CH}\right)$; 6.00 (dd, $J=15.6,7.5 \mathrm{~Hz}, 1 \mathrm{H}, \mathrm{CCH}=\mathrm{CHCH}$ ); 5.89 (d, $\left.J=15.9 \mathrm{~Hz}, 1 \mathrm{H}, \mathrm{CCH}=\mathrm{CHCH}_{2}\right) ; 5.69$ $5.55\left(\mathrm{~m}, 2 \mathrm{H}, \mathrm{CH}\left(\mathrm{OCH}_{3}\right) \mathrm{CH}=\mathrm{CH}, \mathrm{CH}=\mathrm{CHCH}_{3}\right) ; 5.37-5.28\left(\mathrm{~m}, 1 \mathrm{H}, \mathrm{CH}=\mathrm{CHCH}_{3}\right) ; 4.68(\mathrm{~d}, J=$ $\left.6.4 \mathrm{~Hz}, 1 \mathrm{H}, 10 \mathrm{CH}_{2} \mathrm{OCH}_{3}\right) ; 4.48\left(\mathrm{~d}, J=6.4 \mathrm{~Hz}, 1 \mathrm{H}, 1 \mathrm{OCH}_{2} \mathrm{OCH}_{3}\right) ; 4.23-4.13(\mathrm{~m}, 1 \mathrm{H}$, $\left.\mathrm{CHOCH}_{3}\right) ; 3.84\left(\mathrm{~d}, \mathrm{~J}=9.0 \mathrm{~Hz}, 1 \mathrm{H}, \mathrm{CHOCH}_{2} \mathrm{OCH}_{3}\right) ; 3.65$ (dd, J=6.8, $3.5 \mathrm{~Hz}, 1 \mathrm{H}, \mathrm{CHOTES}$ ); 
$3.36\left(\mathrm{~s}, 3 \mathrm{H}, \mathrm{CHOCH}_{3}\right) ; 3.28\left(\mathrm{~s}, 3 \mathrm{H}, \mathrm{CHOCH}_{2} \mathrm{OCH}_{3}\right) ; 3.13(\mathrm{dd}, J=15.0,7.4 \mathrm{~Hz}, 1 \mathrm{H}$, $\left.1 \mathrm{CH}_{2} \mathrm{CHOCH}_{3}\right) ; 3.03$ (dd, $\left.J=15.0,4.2 \mathrm{~Hz}, 1 \mathrm{H}, 1 \mathrm{CH}_{2} \mathrm{CHOCH}_{3}\right) ; 2.59-2.51(\mathrm{~m}, 1 \mathrm{H}$, $\left.1 \mathrm{CH}_{2} \mathrm{CH}=\mathrm{CH}\right) ; 2.45-2.36\left(\mathrm{~m}, 1 \mathrm{H}, 1 \mathrm{CH}_{2} \mathrm{CH}=\mathrm{CH}\right) ; 1.72\left(\mathrm{~d}, J=5.9, \mathrm{~Hz}, 3 \mathrm{H}, \mathrm{CH}=\mathrm{CHCH}_{3}\right) ;$ 0.951 (t, $\left.J=7.8 \mathrm{~Hz}, 9 \mathrm{H}, \mathrm{OSi}\left(\mathrm{CH}_{2} \mathrm{CH}_{3}\right)_{3}\right) ; 0.925$ (s, 3H, CCH $) ; 0.853$ (s, 3H, $\left.\mathrm{CCH}_{3}\right) ; 0.598$ (q, $\left.J=7.9 \mathrm{~Hz}, 6 \mathrm{H}, \mathrm{OSi}\left(\mathrm{CH}_{2} \mathrm{CH}_{3}\right)_{3}\right)$.

${ }^{13} \mathrm{C}$-NMR (151 MHz, $\left.\mathrm{CDCl}_{3}\right)$ : $\delta(\mathrm{ppm}): 164.4(\mathrm{C}=\mathrm{O}) ; 162.8(\mathrm{C}=\mathrm{N}) ; 145.0(\mathrm{CH}) ; 143.2(\mathrm{CH}) ;$ $140.2(\mathrm{CH}) ; 133.0(\mathrm{C}) ; 131.1(\mathrm{CH}) ; 127.9(\mathrm{CH}) ; 114.0(\mathrm{CH}) ; 109.5(\mathrm{CH}) ; 93.6\left(\mathrm{CH}_{2}\right) ; 91.2$ (C); $88.5(\mathrm{C}) ; 81.8(\mathrm{CH}) ; 79.3(\mathrm{CH}) ; 76.5(\mathrm{CH}) ; 56.9\left(\mathrm{CH}_{3}\right) ; 55.7\left(\mathrm{CH}_{3}\right) ; 43.2(\mathrm{C}) ; 34.4\left(\mathrm{CH}_{2}\right)$; $30.5\left(\mathrm{CH}_{2}\right) ; 19.8\left(\mathrm{CH}_{3}\right) ; 19.4\left(\mathrm{CH}_{3}\right) ; 18.0\left(\mathrm{CH}_{3}\right) ; 7.3\left(\mathrm{CH}_{3}\right) ; 5.7\left(\mathrm{CH}_{2}\right)$.

IR(neat): $2953(\mathrm{~m}) ; 2912(\mathrm{~m}) ; 2877(\mathrm{~m}) ; 1716(\mathrm{~m}) ; 1587(\mathrm{~m}) ; 1440(\mathrm{~m}) ; 1359(\mathrm{w}) ; 1234(\mathrm{w})$; $1144(\mathrm{~m}) ; 1090(\mathrm{~s}) ; 1036(\mathrm{~s}) ; 957(\mathrm{~m}) ; 921(\mathrm{~m}) ; 826(\mathrm{~m}) ; 726(\mathrm{~s}) ; 542(\mathrm{w}) \mathrm{cm}^{-1}$.

MS (ESI): $\mathrm{m} / \mathrm{z}(\%): 404.18(100), 579.34(78)\left[\mathrm{M} \mathrm{NH}_{4}\right]^{+}, 500.28$ (51), $372.16(27), 468.25$ (10), $336.15(8), 562.32(3)[M+H]^{+}$.

HRMS (ESI) $\mathrm{m} / \mathrm{z}:[\mathrm{M}+\mathrm{H}]^{+}$Calcd for $\mathrm{C}_{30} \mathrm{H}_{48} \mathrm{NO}_{7} \mathrm{Si}: 562.3200$; found: 562.3188 .

(2E,4S,6S,8Z,12E,14R)-14-methoxy-15-(4-(methoxycarbonyl)oxazol-2-yl)-4(methoxymethoxy)-5,5-dimethylpentadeca-2,8,12-trien-10-yn-6-yl 2-

$((2 R, 3 E, 7 Z, 10 S, 12 S, 13 E)-2-m e t h o x y-12$-(methoxymethoxy)-11,11-dimethyl-10((triethylsilyl)oxy)pentadeca-3,7,13-trien-5-yn-1-yl)oxazole-4-carboxylate (28)

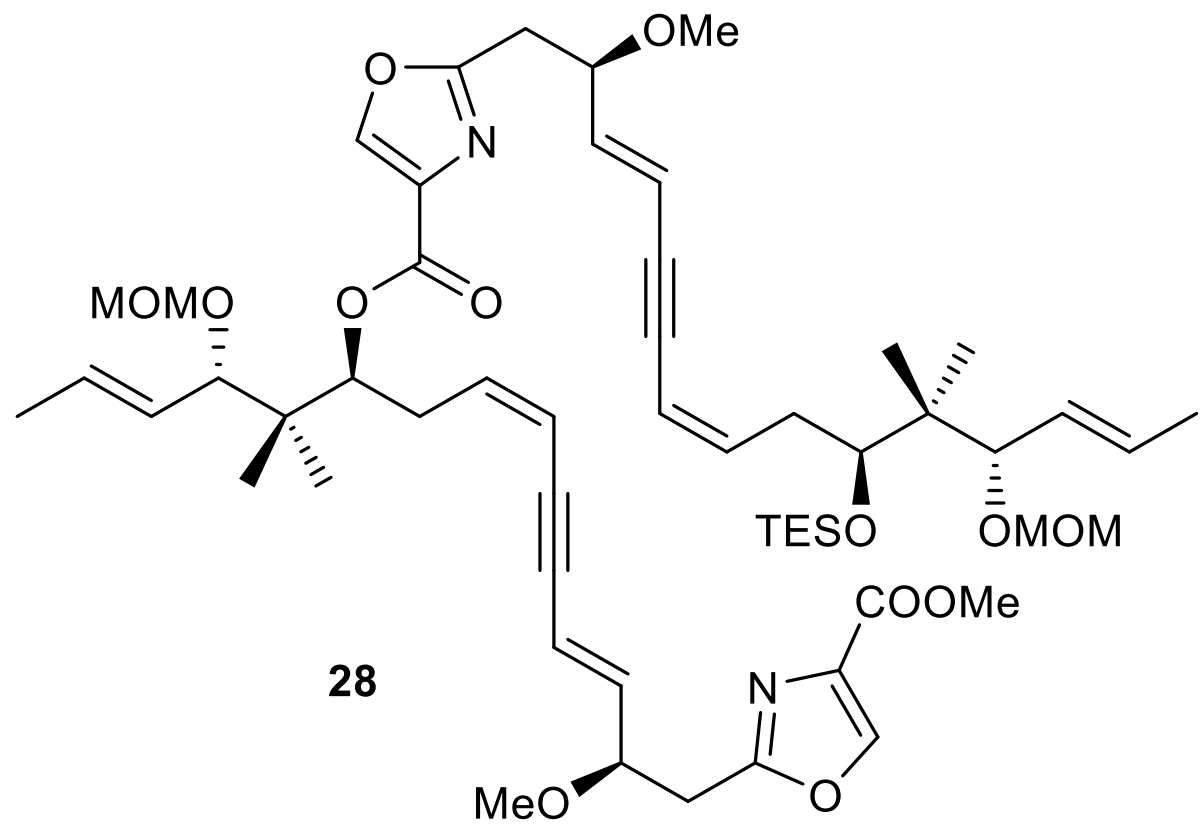

The crude acid 27 (91 mg, $0.162 \mathrm{mmol}, 1.5 \mathrm{eq})$ was dissolved in THF $(5 \mathrm{~mL})$ and treated at room temperature with $\mathrm{NEt}_{3}(90 \mu \mathrm{L}, 0.648 \mathrm{mmol}, 6 \mathrm{eq})$ and 2,4,6-trichlorobenzoyl chloride $(68 \mu \mathrm{L}, 0.432 \mathrm{mmol}, 4 \mathrm{eq})$. The turbid solution was stirred for $2 \mathrm{~h}$ at room temperature and then diluted with toluene $(3 \mathrm{~mL})$ and added dropwise to a solution of alcohol $26(50 \mathrm{mg}$, $0.108 \mathrm{mmol}, 1 \mathrm{eq}$ ) and DMAP (79 mg, $0.648 \mathrm{mmol}, 6 \mathrm{eq}$ ) in toluene $(5 \mathrm{~mL})$. The mixture was stirred overnight at room temperature and then quenched with saturated aqueous $\mathrm{NH}_{4} \mathrm{Cl}$ solution $(15 \mathrm{~mL})$. The aqueous phase was extracted with EtOAc $(3 \times 10 \mathrm{~mL})$. The organic layers were dried over $\mathrm{Na}_{2} \mathrm{SO}_{4}$, filtered and concentrated in vacuo. The residue was purified by flash chromatography (hexane/EtOAc $2: 1$ to $1: 1$ ) to afford the dimer 28 (81 mg, 0.0813 $\mathrm{mmol}, 75 \%$ ) as a slightly yellow oil.

General Data: $\mathrm{C}_{55} \mathrm{H}_{80} \mathrm{~N}_{2} \mathrm{O}_{13} \mathrm{Si}$; FW: 1004.54; TLC: $\mathrm{R}_{\mathrm{f}}=0.30$ (Et $2 \mathrm{O} /$ pentane 2:1); UV (+); Vanillin: black; $[\alpha]_{D}^{20}=+29.65\left(c=1.75, \mathrm{CHCl}_{3}\right)$. 
${ }^{1} \mathrm{H}-\mathrm{NMR}\left(400 \mathrm{MHz}, \mathrm{CDCl}_{3}\right): \delta(\mathrm{ppm}): 8.18(\mathrm{~s}, 1 \mathrm{H}, \mathrm{NC}=\mathrm{CH}) ; 8.06$ (s, $\left.1 \mathrm{H}, \mathrm{NC}=\mathrm{CH}\right) ; 6.10-5.94$ (m, 4H, CH=CH); 5.93-5.84 (m, 2H, $\mathrm{CH}=\mathrm{CH})$; 5.70-5.52 (m, 4H, $\mathrm{CH}=\mathrm{CH}) ; 5.42-5.28(\mathrm{~m}, 2 \mathrm{H}$, $\mathrm{CH}=\mathrm{CH}$ ); 5.25 (app dd, $J=7.6,5.5 \mathrm{~Hz}, 1 \mathrm{H}, \mathrm{CHOC}=\mathrm{O}$ ); 4.65 (dd, $J=6.5,3.0 \mathrm{~Hz}, 2 \mathrm{H}$, $\left.\mathrm{OCH}_{2} \mathrm{OCH}_{3}\right) ; 4.46\left(\mathrm{~d}, J=6.6 \mathrm{~Hz}, 1 \mathrm{H}, 1 \mathrm{OCH} \mathrm{OCH}_{3}\right) ; 4.39\left(\mathrm{~d}, J=6.6 \mathrm{~Hz}, 1 \mathrm{H}, 1 \mathrm{OCH}_{2} \mathrm{OCH}_{3}\right)$; 4.23-4.13 (m, 2H, $\left.\mathrm{CHOCH}_{3}\right) ; 3.90\left(\mathrm{~s}, 3 \mathrm{H}, \mathrm{COOCH}_{3}\right) ; 3.84(\mathrm{~d}, J=9.0 \mathrm{~Hz}, 1 \mathrm{H}$, $\left.\mathrm{CHOCH}_{2} \mathrm{OCH}_{3}\right) ; 3.76\left(\mathrm{~d}, J=9.0 \mathrm{~Hz}, 1 \mathrm{H}, \mathrm{CHOCH}_{2} \mathrm{OCH}_{3}\right) ; 3.66(\mathrm{dd}, J=7.0,3.8 \mathrm{~Hz}, 1 \mathrm{H}$, $\mathrm{CH}_{2} \mathrm{CHOTES}$ ); 3.34 (s, $\left.3 \mathrm{H}, \mathrm{CHOCH}_{3}\right) ; 3.32$ (s, $\left.3 \mathrm{H}, \mathrm{CHOCH}_{3}\right) ; 3.28\left(\mathrm{~s}, 3 \mathrm{H}, \mathrm{CHOCH}_{2} \mathrm{OCH}_{3}\right.$ ); $3.27\left(\mathrm{~s}, 3 \mathrm{H}, \mathrm{CHOCH}_{2} \mathrm{OCH}_{3}\right) ; 3.15-2.93\left(\mathrm{~m}, 4 \mathrm{H}, \mathrm{CH}_{2} \mathrm{CHOCH}_{3}\right) ; 2.72-2.62(\mathrm{~m}, 2 \mathrm{H}$, $\left.\mathrm{CH}_{2} \mathrm{CH}=\mathrm{CH}\right) ; 2.60-2.51\left(\mathrm{~m}, 1 \mathrm{H}, 1 \mathrm{CH}_{2} \mathrm{CH}=\mathrm{CH}\right) ; 2.46-2.36\left(\mathrm{~m}, 1 \mathrm{H}, 1 \mathrm{CH}_{2} \mathrm{CH}=\mathrm{CH}\right) ; 1.71$ (dt, J $\left.=6.4,1.5 \mathrm{~Hz}, 6 \mathrm{H}, \mathrm{CH}=\mathrm{CHCH}_{3}\right) ; 1.03\left(\mathrm{~s}, 3 \mathrm{H}, \mathrm{CCH}_{3}\right) ; 0.966\left(\mathrm{~s}, 3 \mathrm{H}, \mathrm{CCH}_{3}\right) ; 0.945$ (app t, $J=$ $\left.7.9 \mathrm{~Hz}, 9 \mathrm{H}, \mathrm{OSi}\left(\mathrm{CH}_{2} \mathrm{CH}_{3}\right)_{3}\right) ; 0.918\left(\mathrm{~s}, 3 \mathrm{H}, \mathrm{CCH}_{3}\right) ; 0.844$ (s, 3H, CCH $) ; 0.591$ (q, J = 8.0 Hz, $\left.6 \mathrm{H}, \mathrm{OSi}\left(\mathrm{CH}_{2} \mathrm{CH}_{3}\right)_{3}\right)$.

${ }^{13} \mathrm{C}$-NMR $\left(100 \mathrm{MHz}, \mathrm{CDCl}_{3}\right): \delta(\mathrm{ppm}): 162.6(\mathrm{C}=\mathrm{O}) ; 162.5(\mathrm{C}=\mathrm{O}) ; 161.8(\mathrm{C}=\mathrm{N}) ; 160.8(\mathrm{C}=\mathrm{N})$; $144.2(\mathrm{CH}) ; 143.6(\mathrm{CH}) ; 143.2(\mathrm{CH}) ; 140.6(\mathrm{CH}) ; 140.5(\mathrm{CH}) ; 133.6(\mathrm{C}) ; 133.5(\mathrm{C}) ; 132.1$ $(\mathrm{CH}) ; 131.0(\mathrm{CH}) ; 128.0(\mathrm{CH}) ; 127.1(\mathrm{CH}) ; 125.6(\mathrm{CH}) ; 113.8(\mathrm{CH}) ; 113.7(\mathrm{CH}) ; 111.1(\mathrm{CH}) ;$ $109.5(\mathrm{CH})$; $93.6\left(\mathrm{CH}_{2}\right)$; $93.6\left(\mathrm{CH}_{2}\right) ; 91.4(\mathrm{C}) ; 91.2(\mathrm{C}) ; 88.4(\mathrm{C}) ; 88.0(\mathrm{C}) ; 81.6(\mathrm{CH}) ; 81.6$ $(\mathrm{CH}) ; 79.3(\mathrm{CH}) ; 77.4(\mathrm{CH}) ; 77.0(\mathrm{CH}) ; 76.4(\mathrm{CH}) ; 57.0\left(\mathrm{CH}_{3}\right) ; 56.9\left(\mathrm{CH}_{3}\right) ; 56.1\left(\mathrm{CH}_{3}\right) ; 55.7$ $\left(\mathrm{CH}_{3}\right) ; 52.3\left(\mathrm{CH}_{3}\right) ; 43.2(\mathrm{C}) ; 41.8(\mathrm{C}) ; 34.7\left(\mathrm{CH}_{2}\right) ; 34.5\left(\mathrm{CH}_{2}\right) ; 31.4\left(\mathrm{CH}_{2}\right) ; 30.4\left(\mathrm{CH}_{2}\right) ; 19.9$ $\left(\mathrm{CH}_{3}\right) ; 19.8\left(\mathrm{CH}_{3}\right) ; 19.5\left(\mathrm{CH}_{3}\right) ; 19.4\left(\mathrm{CH}_{3}\right) ; 18.1\left(\mathrm{CH}_{3}\right) ; 18.0\left(\mathrm{CH}_{3}\right) ; 7.3\left(\mathrm{CH}_{3}\right) ; 5.7\left(\mathrm{CH}_{2}\right)$.

IR(neat): $2953(\mathrm{~m}) ; 2879(\mathrm{~m}) ; 2284(\mathrm{w}) ; 1737(\mathrm{~m}) ; 1583(\mathrm{~m}) ; 1439(\mathrm{~m}) ; 1318(\mathrm{~m}) ; 1168(\mathrm{~m})$; 1099 (s); 1033 (s); $911(\mathrm{~s}) ; 807(\mathrm{~m}) ; 728(\mathrm{~s}) ; 647(\mathrm{~m}) ; 551(\mathrm{w}) \mathrm{cm}^{-1}$.

MS (ESI): $m / z(\%): 1005.55(100)[M+H]^{+}, 1022.56(760)\left[M+\mathrm{NH}_{4}\right]^{+}, 943.52(45), 973.53$ (28), $847.43(8)$.

HRMS (ESI) $\mathrm{m} / \mathrm{z}:[\mathrm{M}+\mathrm{H}]^{+}$Calcd for $\mathrm{C}_{55} \mathrm{H}_{81} \mathrm{~N}_{2} \mathrm{O}_{13} \mathrm{Si}: 1005.5508$; found: 1005.5594

$\left(16,16^{\prime}\right)$-Bis(methoxymethyl)-(9,10,9',10')-tetradehydridodisorazole C1 (29)

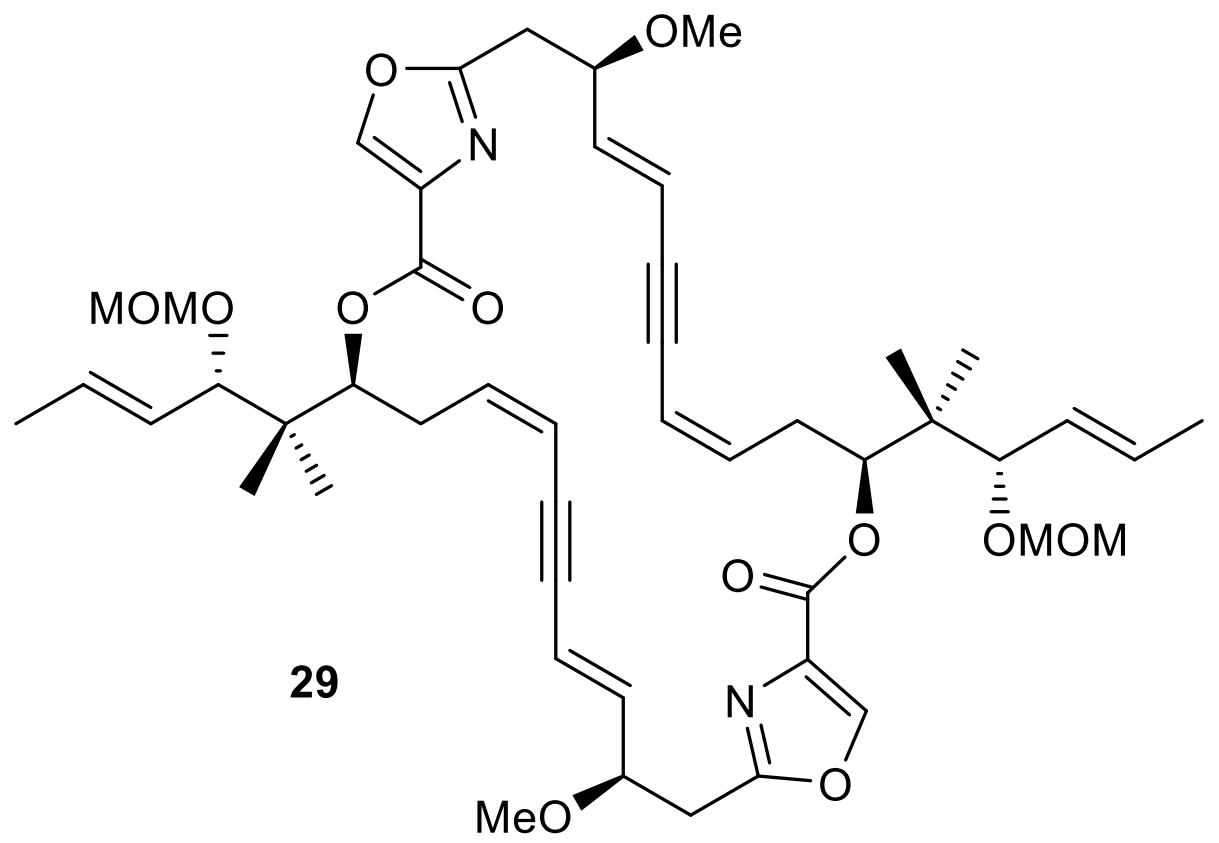

CSA ( $3 \mathrm{mg}, 0.0132 \mathrm{mmol}, 0.2 \mathrm{eq}$ ) was added at $0^{\circ} \mathrm{C}$ to a solution TES protected dimer 28 (67 mg, $0.0661 \mathrm{mmol}, 1 \mathrm{eq})$ in $\mathrm{CH}_{2} \mathrm{Cl}_{2}(2 \mathrm{~mL})$ and $\mathrm{MeOH}(2 \mathrm{~mL})$. The mixture was stirred for $1 \mathrm{~h}$ at $0^{\circ} \mathrm{C}$ under normal atmosphere. Saturated $\mathrm{NaHCO}_{3}$ solution $(10 \mathrm{~mL})$ was added and the layers were separated. The aqueous phase was extracted with $\mathrm{CH}_{2} \mathrm{Cl}_{2}(3 \times 10 \mathrm{~mL})$ and the combined organic extracts were dried over $\mathrm{Na}_{2} \mathrm{SO}_{4}$, filtered and concentrated in vacuo 
giving the deprotected alcohol as a slightly yellow oil, which was used in the next step without further purification.

General Data: $\mathrm{C}_{49} \mathrm{H}_{66} \mathrm{~N}_{2} \mathrm{O}_{13}$; FW: 890.46; TLC: $\mathrm{R}_{\mathrm{f}}=0.30\left(\mathrm{CH}_{2} \mathrm{Cl}_{2} / \mathrm{MeOH} 0: 1\right)$; UV (+); Vanillin: black; $[\alpha]_{D}^{20}=+24.93\left(c=1.4, \mathrm{CHCl}_{3}\right)$.

${ }^{1} \mathrm{H}-\mathrm{NMR}\left(400 \mathrm{MHz}_{\mathrm{CDCl}}\right.$ ): $\delta$ (ppm): $8.18(\mathrm{~s}, 1 \mathrm{H}, \mathrm{NC}=\mathrm{CH}) ; 8.06(\mathrm{~s}, 1 \mathrm{H}, \mathrm{NC}=\mathrm{CH}) ; 6.24-6.14$ $\left(\mathrm{m}, 1 \mathrm{H}, \mathrm{CH}_{2} \mathrm{CH}=\mathrm{CH}\right.$ ); 6.03-6.92 (m, 3H, $\mathrm{CH}=\mathrm{CH}$ ); 5.88 (ddd, J = 15.9, 4.8, $2.0 \mathrm{~Hz}, 2 \mathrm{H}$, $\mathrm{CH}=\mathrm{CH}) ; 5.72-5.60(\mathrm{~m}, 3 \mathrm{H}, \mathrm{CH}=\mathrm{CH}) ; 5.56(\mathrm{~d}, \mathrm{~J}=10.1 \mathrm{~Hz}, 1 \mathrm{H}, \mathrm{CH}=\mathrm{CH}) ; 5.44-5.32(\mathrm{~m}, 2 \mathrm{H}$, $\mathrm{CH}=\mathrm{CH}) ; 5.25(\mathrm{dd}, J=8.0,5.2 \mathrm{~Hz}, 1 \mathrm{H}, \mathrm{CHOC}=0) ; 4.70(\mathrm{dd}, J=15.0,6.7 \mathrm{~Hz}, 1 \mathrm{H}$, $\left.10 \mathrm{CH}_{2} \mathrm{OCH}_{3}\right) ; 4.45\left(\mathrm{t}, J=6.7 \mathrm{~Hz}, 1 \mathrm{H}, 10 \mathrm{CH}_{2} \mathrm{OCH}_{3}\right) ; 4.65$ (dd, $J=6.5,1.8 \mathrm{~Hz}, 1 \mathrm{H}$, $\left.\mathrm{CHOCH}_{2} \mathrm{OCH}_{3}\right) ; 4.47\left(\mathrm{~d}, J=6.6 \mathrm{~Hz}, 1 \mathrm{H}, \mathrm{CHOCH}_{2} \mathrm{OCH}_{3}\right) ; 4.39(\mathrm{~d}, J=6.6 \mathrm{~Hz}, 1 \mathrm{H}$, $\left.\mathrm{CHOCH}_{2} \mathrm{OCH}_{3}\right) ; 4.23-4.13\left(\mathrm{~m}, 2 \mathrm{H}, \mathrm{CHOCH}_{3}\right) ; 3.90\left(\mathrm{~s}, 3 \mathrm{H}, \mathrm{COOCH}_{3}\right) ; 3.89(\mathrm{~m}, 1 \mathrm{H}$, $\mathrm{CHOCH}_{2} \mathrm{OCH}_{3}$ ); $3.76\left(\mathrm{~d}, J=9.0 \mathrm{~Hz}, 1 \mathrm{H}, \mathrm{CHOCH}_{2} \mathrm{OCH}_{3}\right) ; 3.68$ (dd, $J=10.3,2.4 \mathrm{~Hz}, 1 \mathrm{H}$, $\mathrm{CHOH}) ; 3.38\left(\mathrm{~s}, 3 \mathrm{H}, \mathrm{CHOCH}_{3}\right) ; 3.32\left(\mathrm{~s}, 3 \mathrm{H}, \mathrm{CHOCH}_{3}\right) ; 3.28\left(\mathrm{~s}, 3 \mathrm{H}, \mathrm{CHOCH}_{2} \mathrm{OCH}_{3}\right) ; 3.27$ (s, 3H, $\left.\mathrm{CHOCH}_{2} \mathrm{OCH}_{3}\right) ; 3.15-2.94\left(\mathrm{~m}, 4 \mathrm{H}, \mathrm{CH}_{2} \mathrm{CHOCH}_{3}\right) ; 2.72-2.62\left(\mathrm{~m}, 2 \mathrm{H}, \mathrm{CH} \mathrm{H}_{2} \mathrm{CH}=\mathrm{CH}\right)$; 2.58-2.48 (m, $\left.1 \mathrm{H}, 1 \mathrm{CH}_{2} \mathrm{CH}=\mathrm{CH}\right) ; 2.39-2.28\left(\mathrm{~m}, 1 \mathrm{H}, 1 \mathrm{CH}_{2} \mathrm{CH}=\mathrm{CH}\right) ; 1.74(\mathrm{dd}, J=6.5,1.4 \mathrm{~Hz}$, $3 \mathrm{H}, \mathrm{CH}=\mathrm{CHCH}_{3}$ ); 1.71 (dd, $\left.J=6.5,1.4 \mathrm{~Hz}, 3 \mathrm{H}, \mathrm{CH}=\mathrm{CHCH}_{3}\right) ; 1.03$ (s, 3H, CCH $) ; 0.968$ (s, $\left.3 \mathrm{H}, \mathrm{CCH}_{3}\right) ; 0.921\left(\mathrm{~s}, 3 \mathrm{H}, \mathrm{CCH}_{3}\right) ; 0.868\left(\mathrm{~s}, 3 \mathrm{H}, \mathrm{CCH}_{3}\right)$.

${ }^{13} \mathrm{C}-N M R\left(100 \mathrm{MHz}, \mathrm{CDCl}_{3}\right)$ : $\delta(\mathrm{ppm})$ : $162.6(\mathrm{C}=\mathrm{O}) ; 162.5(\mathrm{C}=\mathrm{O}) ; 161.8(\mathrm{C}=\mathrm{N}) ; 160.8(\mathrm{C}=\mathrm{N})$; $144.2(\mathrm{CH}) ; 143.6(\mathrm{CH}) ; 143.2(\mathrm{CH}) ; 140.6(\mathrm{CH}) ; 140.4(\mathrm{CH}) ; 140.4(\mathrm{CH}) ; 133.6(\mathrm{C}) ; 133.5$ $(\mathrm{C}) ; 132.1(\mathrm{CH}) ; 132.0(\mathrm{CH}) ; 127.1(\mathrm{CH}) ; 126.8(\mathrm{CH}) ; 113.9(\mathrm{CH}) ; 113.7(\mathrm{CH}) ; 111.1(\mathrm{CH})$; $109.8(\mathrm{CH})$; $93.8\left(\mathrm{CH}_{2}\right)$; $93.6\left(\mathrm{CH}_{2}\right) ; 91.4(\mathrm{C}) ; 91.2(\mathrm{C}) ; 88.3(\mathrm{C}) ; 88.0(\mathrm{C}) ; 84.7(\mathrm{CH}) ; 81.4$ $(\mathrm{CH}) ; 79.3(\mathrm{CH}) ; 77.4(\mathrm{CH}) ; 77.0(\mathrm{CH}) ; 76.1(\mathrm{CH}) ; 57.0\left(\mathrm{CH}_{3}\right) ; 56.9\left(\mathrm{CH}_{3}\right) ; 56.2\left(\mathrm{CH}_{3}\right) ; 56.1$ $\left(\mathrm{CH}_{3}\right) ; 52.3\left(\mathrm{CH}_{3}\right) ; 41.8(\mathrm{C}) ; 41.0(\mathrm{C}) ; 34.7\left(\mathrm{CH}_{2}\right) ; 34.7\left(\mathrm{CH}_{2}\right) ; 32.8\left(\mathrm{CH}_{2}\right) ; 31.4\left(\mathrm{CH}_{2}\right) ; 21.1$ $\left(\mathrm{CH}_{3}\right) ; 19.9\left(\mathrm{CH}_{3}\right) ; 19.8\left(\mathrm{CH}_{3}\right) ; 19.5\left(\mathrm{CH}_{3}\right) ; 18.1\left(\mathrm{CH}_{3}\right) ; 18.0\left(\mathrm{CH}_{3}\right)$.

IR(neat): 3658 (br); 2980 (s); $2890(\mathrm{~m}) ; 1737(\mathrm{~m}) ; 1584(\mathrm{~m}) ; 1463(\mathrm{~m}) ; 1380(\mathrm{~m}) ; 1258$ (m); 1143 (s); 1098 (s); 1031 (s); $969(\mathrm{~m}) ; 919(\mathrm{~m}) ; 805(\mathrm{~m}) ; 732(\mathrm{~m}) ; 542(\mathrm{w}) \mathrm{cm}^{-1}$.

MS (ESI): $m / z(\%): 908.49(100)\left[\mathrm{M}_{+} \mathrm{NH}_{4}\right]^{+}, 891.46(98)[\mathrm{M}+\mathrm{H}]^{+}, 859.44$ (35), 733.33 (7), 829.43 (5).

HRMS (ESI) $m / z:[M+H]^{+}$Calcd for $\mathrm{C}_{49} \mathrm{H}_{67} \mathrm{~N}_{2} \mathrm{O}_{13}$ : 891.4643; found: 891.4768 .

The crude deprotected alcohol was dissolved in THF $(1.5 \mathrm{~mL})$ and treated at room temperature with $\mathrm{LiOH}\left(1 \mathrm{M}\right.$ in $\left.\mathrm{H}_{2} \mathrm{O}, 0.165 \mathrm{~mL}, 0.165 \mathrm{mmol}, 2.5 \mathrm{eq}\right)$. The mixture was stirred overnight at room temperature and neutralized with $1 \mathrm{M} \mathrm{HCl}(1 \mathrm{~mL})$. The aqueous phase was extracted with $\mathrm{Et}_{2} \mathrm{O}(3 \times 3 \mathrm{~mL})$ and the organic extracts were dried over $\mathrm{Na}_{2} \mathrm{SO}_{4}$, filtered and concentrated in vacuo to give the seco-acid as a yellow wax, which was used without further purification.

General Data: $\mathrm{C}_{48} \mathrm{H}_{64} \mathrm{~N}_{2} \mathrm{O}_{13}$; FW: 876.44; TLC: UV (+); Vanillin: grey; $[\alpha]_{D}^{20}=+17.4(c=1.3$, $\left.\mathrm{CHCl}_{3}\right)$.

${ }^{1} \mathrm{H}-\mathrm{NMR}\left(400 \mathrm{MHz}, \mathrm{CDCl}_{3}\right): \delta(\mathrm{ppm}): 8.24(\mathrm{~s}, 1 \mathrm{H}, \mathrm{NC}=\mathrm{CH}) ; 8.07$ (s, $\left.1 \mathrm{H}, \mathrm{NC}=\mathrm{CH}\right) ; 6.24-6.14$ $\left(\mathrm{m}, 1 \mathrm{H}, \mathrm{CH}_{2} \mathrm{CH}=\mathrm{CH}\right) ;$ 6.04-5.93 (m, 3H, $\left.\mathrm{CH}=\mathrm{CH}\right) ; 5.93-5.81(\mathrm{~m}, 2 \mathrm{H}, \mathrm{CH}=\mathrm{CH})$; 5.73-5.60 (m, $3 \mathrm{H}, \mathrm{CH}=\mathrm{CH}) ; 5.55(\mathrm{~d}, J=10.5 \mathrm{~Hz}, 1 \mathrm{H}, \mathrm{CH}=\mathrm{CH}) ; 5.44-5.32(\mathrm{~m}, 2 \mathrm{H}, \mathrm{CH}=\mathrm{CH}) ; 5.25$ (dd, $J=$ 8.6, $4.3 \mathrm{~Hz}, 1 \mathrm{H}, \mathrm{CHOC}=\mathrm{O}) ; 4.67\left(\mathrm{~d}, J=6.6 \mathrm{~Hz}, 2 \mathrm{H}, \mathrm{OCH}_{2} \mathrm{OCH}_{3}\right) ; 4.49(\mathrm{~d}, J=6.6 \mathrm{~Hz}, 1 \mathrm{H}$, $\left.10 \mathrm{CH}_{2} \mathrm{OCH}_{3}\right) ; 4.41\left(\mathrm{~d}, J=6.6 \mathrm{~Hz}, 1 \mathrm{H}, 1 \mathrm{OCH}_{2} \mathrm{OCH}_{3}\right) ; 4.23-4.14\left(\mathrm{~m}, 2 \mathrm{H}, \mathrm{CHOCH}_{3}\right) ; 3.92(\mathrm{~d}$, $\left.J=8.8 \mathrm{~Hz}, 1 \mathrm{H}, \mathrm{CHOCH}_{2} \mathrm{OCH}_{3}\right) ; 3.76\left(\mathrm{~d}, J=9.1 \mathrm{~Hz}, 1 \mathrm{H}, \mathrm{CHOCH}_{2} \mathrm{OCH}_{3}\right) ; 3.70(\mathrm{dd}, J=10.3$, $2.6 \mathrm{~Hz}, 1 \mathrm{H}, \mathrm{CHOH}) ; 3.39\left(\mathrm{~s}, 3 \mathrm{H}, \mathrm{CHOCH}_{3}\right) ; 3.34\left(\mathrm{~s}, 3 \mathrm{H}, \mathrm{CHOCH}_{3}\right) ; 3.30(\mathrm{~s}, 3 \mathrm{H}$, $\mathrm{CHOCH}_{2} \mathrm{OCH}_{3}$ ); 3.27 (s, 3H, $\left.\mathrm{CHOCH}_{2} \mathrm{OCH}_{3}\right) ; 3.19-2.94\left(\mathrm{~m}, 4 \mathrm{H}, \mathrm{CH}_{2} \mathrm{CHOCH}_{3}\right) ; 2.75-2.61$ (m, $\left.2 \mathrm{H}, \mathrm{CH}_{2} \mathrm{CH}=\mathrm{CH}\right) ; 2.59-2.49\left(\mathrm{~m}, 1 \mathrm{H}, 1 \mathrm{CH}_{2} \mathrm{CH}=\mathrm{CH}\right) ; 2.42-2.29\left(\mathrm{~m}, 1 \mathrm{H}, 1 \mathrm{CH}_{2} \mathrm{CH}=\mathrm{CH}\right)$; $1.74\left(\mathrm{dd}, J=6.5,1.4 \mathrm{~Hz}, 3 \mathrm{H}, \mathrm{CH}=\mathrm{CHCH}_{3}\right) ; 1.71$ (dd, $\left.J=6.5,1.4 \mathrm{~Hz}, 3 \mathrm{H}, \mathrm{CH}=\mathrm{CHCH}_{3}\right) ; 1.03$ (s, 3H, CCH 3$) ; 0.975$ (s, 3H, CCH$) ; 0.927\left(\mathrm{~s}, 3 \mathrm{H}, \mathrm{CCH}_{3}\right) ; 0.877$ (s, 3H, $\left.\mathrm{CCH}_{3}\right)$.

${ }^{13} \mathrm{C}-N M R\left(100 \mathrm{MHz}, \mathrm{CDCl}_{3}\right)$ : $\delta(\mathrm{ppm})$ : $163.7(\mathrm{C}=\mathrm{O}) ; 162.6(\mathrm{C}=\mathrm{O}) ; 162.5(\mathrm{C}=\mathrm{N}) ; 160.7(\mathrm{C}=\mathrm{N})$; $144.8(\mathrm{CH}) ; 143.7(\mathrm{CH}) ; 143.1(\mathrm{CH}) ; 140.5(\mathrm{CH}) ; 140.3(\mathrm{CH}) ; 133.5(\mathrm{C}) ; 133.2(\mathrm{C}) ; 132.2$ 
$(\mathrm{CH}) ; 132.1(\mathrm{CH}) ; 127.1(\mathrm{CH}) ; 126.8(\mathrm{CH}) ; 125.7(\mathrm{CH}) ; 113.9(\mathrm{CH}) ; 113.8(\mathrm{CH}) ; 111.2(\mathrm{CH})$; $109.9(\mathrm{CH})$; $93.8\left(\mathrm{CH}_{3}\right)$; $93.5\left(\mathrm{CH}_{3}\right) ; 91.4(\mathrm{C}) ; 91.2(\mathrm{C}) ; 88.3(\mathrm{C}) ; 88.0(\mathrm{C}) ; 84.7(\mathrm{CH}) ; 81.6$ $(\mathrm{CH}) ; 79.4(\mathrm{CH}) ; 79.3(\mathrm{CH}) ; 77.4(\mathrm{CH}) ; 76.3(\mathrm{CH}) ; 57.0\left(\mathrm{CH}_{3}\right) ; 56.9\left(\mathrm{CH}_{3}\right) ; 56.2\left(\mathrm{CH}_{3}\right) ; 56.1$ $\left(\mathrm{CH}_{3}\right) ; 41.8(\mathrm{C}) ; 41.0(\mathrm{C}) ; 34.6\left(\mathrm{CH}_{2}\right) ; 34.5\left(\mathrm{CH}_{2}\right) ; 30.5\left(\mathrm{CH}_{2}\right) ; 29.8\left(\mathrm{CH}_{2}\right) ; 21.1\left(\mathrm{CH}_{3}\right) ; 19.9$ $\left(\mathrm{CH}_{3}\right) ; 19.9\left(\mathrm{CH}_{3}\right) ; 19.4\left(\mathrm{CH}_{3}\right) ; 18.1\left(\mathrm{CH}_{3}\right) ; 18.1\left(\mathrm{CH}_{3}\right)$.

IR(neat): 3658 (br); 2980 (s); 2923 (s); 2328 (w); 1719 (m); 1584 (m); 1461 (m); 1377 (m); $1251(\mathrm{~m}) ; 1146(\mathrm{~m}) ; 1098(\mathrm{~s}) ; 1032(\mathrm{~s}) ; 969(\mathrm{~s}) ; 909(\mathrm{~m}) ; 818(\mathrm{w}) ; 734(\mathrm{~s}) ; 542(\mathrm{w}) \mathrm{cm}^{-1}$.

MS (ESI): $m / z(\%): 877.44(100)[\mathrm{M}+\mathrm{H}]^{+}, 894.47(77)\left[\mathrm{M}^{+} \mathrm{NH}_{4}\right]^{+}, 845.42$ (49), $719.31(30)$, 783.38 (23).

HRMS (ESI) $\mathrm{m} / \mathrm{z}:[\mathrm{M}+\mathrm{H}]^{+}$Calcd for $\mathrm{C}_{48} \mathrm{H}_{65} \mathrm{~N}_{2} \mathrm{O}_{13}: 877.4487$; found: 877.4473 .

The crude seco-acid was dissolved in THF $(5 \mathrm{~mL})$ and treated at room temperature with $\mathrm{NEt}_{3}(184 \mu \mathrm{L}, 1.32 \mathrm{mmol}, 20 \mathrm{eq})$ and 2,4,6-trichlorobenzoyl chloride $(103 \mu \mathrm{L}, 0.661 \mathrm{mmol}$, $10 \mathrm{eq}$ ). The turbid solution was stirred for $2 \mathrm{~h}$ at room temperature and then diluted with toluene $(3 \mathrm{~mL})$ and added dropwise to a solution of DMAP (323 mg, $2.64 \mathrm{mmol}, 40 \mathrm{eq})$ in toluene $(80 \mathrm{~mL})$. The mixture was stirred overnight at room temperature and then quenched with saturated aqueous $\mathrm{NH}_{4} \mathrm{Cl}$ solution $(20 \mathrm{~mL})$ and water $(20 \mathrm{~mL})$ and the aqueous phase was extracted with EtOAc $(3 \times 40 \mathrm{~mL})$. The organic layers were dried over $\mathrm{Na}_{2} \mathrm{SO}_{4}$, filtered and concentrated in vacuo. The residue was purified by flash chromatography (hexane/EtOAc 2:1 to 1:1) to afford the macrocycle 29 ( $40 \mathrm{mg}, 0.0462 \mathrm{mmol}, 70 \%$ from 28) as a slightly yellow oil.

General Data: $\mathrm{C}_{48} \mathrm{H}_{62} \mathrm{~N}_{2} \mathrm{O}_{12}$; FW: 858.43; TLC: $\mathrm{Rf}=0.50\left(\mathrm{CH}_{2} \mathrm{Cl}_{2} / \mathrm{MeOH}^{2} 0: 1\right)$; UV (+); Vanillin: black; $[\alpha]_{D}^{20}=+140.2\left(c=0.5, \mathrm{CHCl}_{3}\right)$.

${ }^{1} \mathrm{H}-\mathrm{NMR}\left(600 \mathrm{MHz}, \mathrm{CDCl}_{3}\right): \delta$ (ppm): $8.04(\mathrm{~s}, 2 \mathrm{H}, \mathrm{NC}=\mathrm{CH}) ; 6.02-5.90(\mathrm{~m}, 4 \mathrm{H}, \mathrm{CH}=\mathrm{CH}) ; 5.70-$ $5.60(\mathrm{~m}, 4 \mathrm{H}, \mathrm{CH}=\mathrm{CH}) ; 5.51(\mathrm{~d}, J=10.3 \mathrm{~Hz}, 2 \mathrm{H}, \mathrm{CH}=\mathrm{CH}) ; 5.44-5.36(\mathrm{~m}, 2 \mathrm{H}, \mathrm{CH}=\mathrm{CH}) ; 5.34$ (dd, $J=11.0,2.3 \mathrm{~Hz}, 2 \mathrm{H}, \mathrm{CHOC}=0) ; 4.67\left(\mathrm{~d}, J=6.6 \mathrm{~Hz}, 1 \mathrm{H}, 1 \mathrm{OCH} \mathrm{H}_{2} \mathrm{OCH}_{3}\right) ; 4.42(\mathrm{~d}, J=6.6$ $\left.\mathrm{Hz}, 1 \mathrm{H}, 1 \mathrm{OCH}_{2} \mathrm{OCH}_{3}\right) ; 4.17-4.08\left(\mathrm{~m}, 2 \mathrm{H}, \mathrm{CHOCH}_{3}\right) ; 3.73\left(\mathrm{~d}, J=9.2 \mathrm{~Hz}, 2 \mathrm{H}, \mathrm{CHOCH}_{2} \mathrm{OCH}_{3}\right)$; $3.36\left(\mathrm{~s}, 6 \mathrm{H}, \mathrm{CHOCH}_{3}\right) ; 3.34\left(\mathrm{~s}, 6 \mathrm{H}, \mathrm{CHOCH}_{2} \mathrm{OCH}_{3}\right)$; 3.32-3.26 (m, 2H, $\left.\mathrm{CH}_{2} \mathrm{CH}=\mathrm{CH}\right)$; 3.06$2.88\left(\mathrm{~m}, 4 \mathrm{H}, \mathrm{CH}_{2} \mathrm{CHOCH}_{3}\right) ; 2.43-2.35\left(\mathrm{~m}, 2 \mathrm{H}, \mathrm{CH}_{2} \mathrm{CH}=\mathrm{CH}\right) ; 1.74(\mathrm{dd}, J=6.1,1.2 \mathrm{~Hz}, 6 \mathrm{H}$, $\left.\mathrm{CH}=\mathrm{CHCH}_{3}\right) ; 1.04\left(\mathrm{~s}, 6 \mathrm{H}, \mathrm{CCH}_{3}\right) ; 1.00\left(\mathrm{~s}, 6 \mathrm{H}, \mathrm{CCH}_{3}\right)$.

${ }^{13} \mathrm{C}$-NMR (151 MHz, $\left.\mathrm{CDCl}_{3}\right): \delta(\mathrm{ppm}): 161.8(\mathrm{C}=\mathrm{O}) ; 160.7(\mathrm{C}=\mathrm{N}) ; 143.4(\mathrm{CH}) ; 141.3(\mathrm{CH})$; $140.4(\mathrm{CH}) ; 133.8(\mathrm{C}) ; 132.0(\mathrm{CH}) ; 127.2(\mathrm{CH}) ; 113.7(\mathrm{CH}) ; 112.2(\mathrm{CH}) ; 93.8\left(\mathrm{CH}_{2}\right) ; 91.0$ (C); $87.9(\mathrm{C}) ; 81.5(\mathrm{CH}) ; 79.7(\mathrm{CH}) ; 76.4(\mathrm{CH}) ; 57.0\left(\mathrm{CH}_{3}\right) ; 56.2\left(\mathrm{CH}_{3}\right) ; 41.6(\mathrm{C}) ; 34.5\left(\mathrm{CH}_{2}\right)$; $31.5\left(\mathrm{CH}_{2}\right) ; 19.9\left(\mathrm{CH}_{3}\right) ; 19.6\left(\mathrm{CH}_{3}\right) ; 18.1\left(\mathrm{CH}_{3}\right)$.

IR(neat): 3172 (w); 3180 (w); 2930 (m); 2855 (m); 1736 (m); $1649(\mathrm{~m}) ; 1584(\mathrm{~m}) ; 1450(\mathrm{~m})$; $1368(\mathrm{~m}) ; 1216(\mathrm{~m}) ; 1140(\mathrm{~m}) ; 1103(\mathrm{~s}) ; 1031(\mathrm{~s}) ; 973(\mathrm{~m}) ; 921(\mathrm{~m}) ; 830(\mathrm{~m}) ; 752(\mathrm{~s}) \mathrm{cm}^{-1}$. MS (ESI): $m / z(\%): 859.44(100)[M+\mathrm{H}]^{+}, 876.46(42)\left[M+\mathrm{NH}_{4}\right]^{+}, 797.40(16), 735.36$ (3). HRMS (ESI) $m / z:[M+H]^{+}$Calcd for $\mathrm{C}_{48} \mathrm{H}_{65} \mathrm{~N}_{2} \mathrm{O}_{13}: 859.4381$; found: 859.4464 . 
$(16,16$ ')-Bis(methoxymethyl)-disorazole C1 (30)

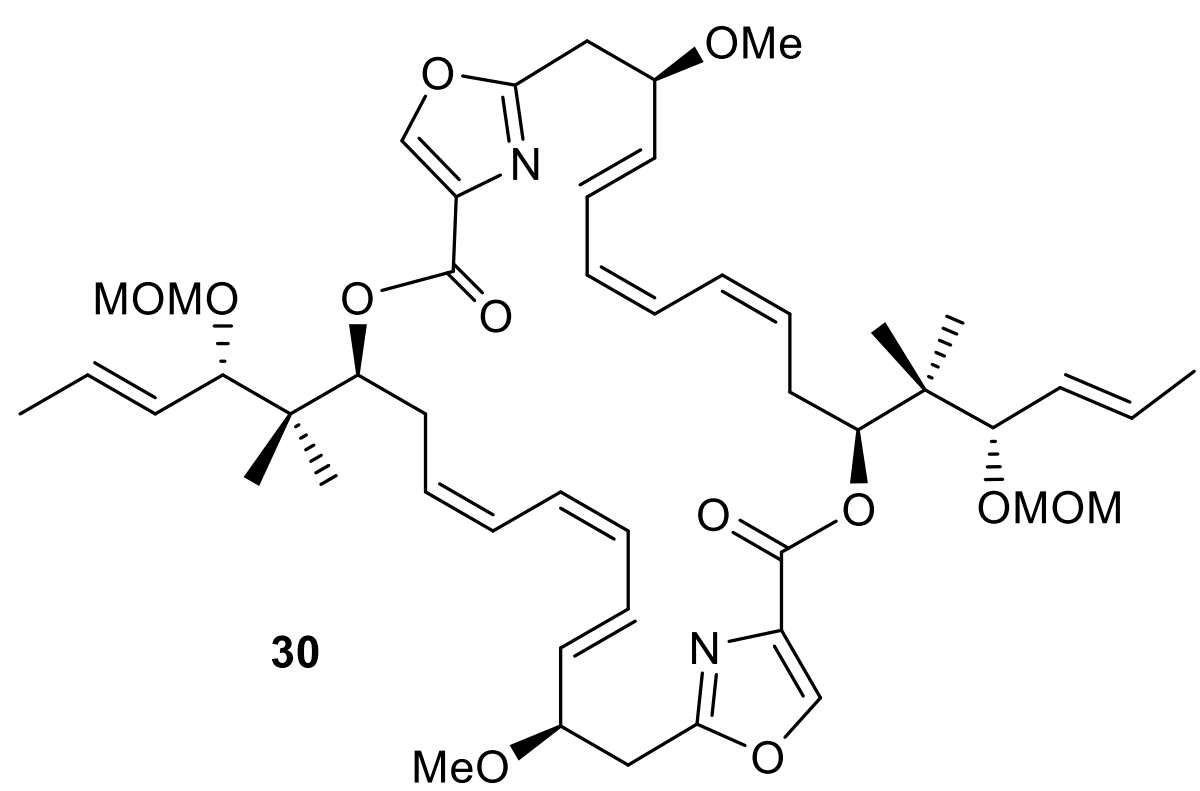

Nitrogen is bubbled for 15 min through a suspension of Zinc $(5 \mathrm{~g}, 45.88 \mathrm{mmol})$ in $\mathrm{H}_{2} \mathrm{O}$ (30 $\mathrm{mL})$ and then $\mathrm{Cu}(\mathrm{OAc})_{2} \cdot \mathrm{H}_{2} \mathrm{O}(500 \mathrm{mg}, 2.5 \mathrm{mmol})$ was added at room temperature and after 15 min $\mathrm{AgNO}_{3}(500 \mathrm{mg}, 2.94 \mathrm{mmol}$ ) was added (exothermic reaction). The mixture was stirred for $30 \mathrm{~min}$ at room temperature, filtered by suction and washed with $\mathrm{H}_{2} \mathrm{O}(40 \mathrm{~mL})$, $\mathrm{MeOH}(30 \mathrm{~mL})$, acetone $(30 \mathrm{~mL})$ and $\mathrm{Et}_{2} \mathrm{O}(30 \mathrm{~mL})$. This activated zinc solids were added to a solution of 29 (40 mg, $0.0466 \mathrm{mmol})$ in $\mathrm{MeOH} / \mathrm{H}_{2} \mathrm{O} 1: 1(20 \mathrm{~mL})$. The mixture was stirred for $24 \mathrm{~h}$ at $50^{\circ} \mathrm{C}$, then filtered on a pad of silica with $\mathrm{MeOH}$ washes. The filtrate was concentrated in vacuo and the residue was purified by flash chromatography $\left(\mathrm{CH}_{2} \mathrm{Cl}_{2} / \mathrm{MeOH}\right.$ $70: 1)$ to afford 30 (26 mg, 0.0302, 65\%) as a colorless wax.

General Data: $\mathrm{C}_{48} \mathrm{H}_{66} \mathrm{~N}_{2} \mathrm{O}_{12}$; FW: 862.46; TLC: $\mathrm{R}_{f}=0.40\left(\mathrm{CH}_{2} \mathrm{Cl}_{2} / \mathrm{MeOH} 50: 1\right)$; UV (+); Vanillin: dark green; $[\alpha]_{D}^{20}=-1.2\left(c=0.55, \mathrm{CHCl}_{3}\right)$.

${ }^{1} \mathrm{H}-\mathrm{NMR}\left(600 \mathrm{MHz}, \mathrm{CDCl}_{3}\right)$ : $\delta$ (ppm): 7.89 (s, 2H, NC=CH); 6.42 (dd, $J=15.3,11.4 \mathrm{~Hz}, 2 \mathrm{H}$, $\mathrm{CH}(\mathrm{OCH} 3) \mathrm{CH}=\mathrm{CH}$ ); 6.32 (app t, $J=11.3 \mathrm{~Hz}, 2 \mathrm{H}, \mathrm{CH} 2 \mathrm{CH}=\mathrm{CH}) ; 6.18$ (dd, $J=11.4,11.1 \mathrm{~Hz}$, $2 \mathrm{H}, \mathrm{CHCH}=\mathrm{CHCH}$ ); 5.90 (dd, $J=11.2,11.0 \mathrm{~Hz}, 2 \mathrm{H}, \mathrm{CHCH}=\mathrm{CHCH}) ; 5.63(\mathrm{dd}, J=15.4,6.5$ $\mathrm{Hz}, 2 \mathrm{H}, \mathrm{CH} 3 \mathrm{CH}=\mathrm{CH}$ ); 5.53 (dd, $J=15.1,8.5 \mathrm{~Hz}, 2 \mathrm{H}, \mathrm{CH} 3 \mathrm{CH}=\mathrm{CH}) ; 5.51$ (dd, $J=16.6,8.9$ $\mathrm{Hz}, 2 \mathrm{H}, \mathrm{CHOC}=\mathrm{O}$ ); 5.37 (ddd, $J=15.4,9.1,1.5 \mathrm{~Hz}, 2 \mathrm{H}, \mathrm{CH}(\mathrm{OCH} 3) \mathrm{CH}=\mathrm{CH}$ ); 5.26 (dd, $J=$ 11.2, $2.2 \mathrm{~Hz}, 2 \mathrm{H}, \mathrm{CH} 2 \mathrm{CH}=\mathrm{CH}) ; 4.65\left(\mathrm{~d}, J=6.7 \mathrm{~Hz}, 1 \mathrm{H}, 1 \mathrm{OCH} \mathrm{OCH}_{3}\right) ; 4.39$ (d, $J=6.7 \mathrm{~Hz}$, $\left.1 \mathrm{H}, 1 \mathrm{OCH}_{2} \mathrm{OCH}_{3}\right) ; 4.13$ (ddd, $\left.J=12.8,7.0,5.0 \mathrm{~Hz}, 2 \mathrm{H}, \mathrm{CHOCH} 3\right) ; 3.71(\mathrm{~d}, J=9.0 \mathrm{~Hz}, 2 \mathrm{H}$, $\mathrm{CHOH}) ; 3.33\left(\mathrm{~s}, 6 \mathrm{H}, \mathrm{CHOCH}_{2} \mathrm{OCH}_{3}\right) ; 3.26\left(\mathrm{~s}, 6 \mathrm{H}, \mathrm{CHOCH}_{3}\right) ; 3.13(\mathrm{dd}, \mathrm{J}=13.8,5.9 \mathrm{~Hz}, 2 \mathrm{H}$, $\mathrm{CH}_{2} \mathrm{CHOCH}_{3}$ ); 2.79 (dd, $J=14.6,7.5 \mathrm{~Hz}, 2 \mathrm{H}, \mathrm{CH}_{2} \mathrm{CHOCH}_{3}$ ); 2.57 (ddd, $J=14.7,10.1,5.1$ $\mathrm{Hz}, \mathrm{CH}_{2} \mathrm{CH}=\mathrm{CH}$ ); 2.43 (dd, $\left.J=14.9,6.3 \mathrm{~Hz}, 2 \mathrm{H}, \mathrm{CH}_{2} \mathrm{CH}=\mathrm{CH}\right) ; 1.72(\mathrm{dd}, J=6.3,1.3 \mathrm{~Hz}, 3 \mathrm{H}$, $\left.\mathrm{CH}=\mathrm{CHCH}_{3}\right) ; 1.01$ (s, 6H, $\left.\mathrm{CCH}_{3}\right) ; 0.946\left(\mathrm{~s}, 6 \mathrm{H}, \mathrm{CCH}_{3}\right)$.

${ }^{13} \mathrm{C}-N M R\left(151 \mathrm{MHz}, \mathrm{CDCl}_{3}\right)$ : $\delta(\mathrm{ppm}): 162.3(\mathrm{C}=\mathrm{O}) ; 160.7(\mathrm{C}=\mathrm{N}) ; 143.3(\mathrm{CH}) ; 133.5(\mathrm{C})$; $133.3(\mathrm{CH}) ; 132.1(\mathrm{CH}) ; 130.0(\mathrm{CH}) ; 129.1(\mathrm{CH}) ; 128.2(\mathrm{CH}) ; 127.2(\mathrm{CH}) ; 125.7(\mathrm{CH}) ; 125.5$ $(\mathrm{CH}) ; 93.6\left(\mathrm{CH}_{2}\right) ; 81.7(\mathrm{CH}) ; 80.0(\mathrm{CH}) ; 77.4^{*}(\mathrm{CH}) ; 56.7\left(\mathrm{CH}_{3}\right) ; 56.1\left(\mathrm{CH}_{3}\right) ; 41.7(\mathrm{C}) ; 35.2$ $\left(\mathrm{CH}_{2}\right) ; 29.9\left(\mathrm{CH}_{2}\right) ; 20.1\left(\mathrm{CH}_{3}\right) ; 19.8\left(\mathrm{CH}_{3}\right) ; 18.1\left(\mathrm{CH}_{3}\right)$.

*obscured by $\mathrm{CDCl}_{3}$

IR(neat): 2927 (m); $2855(\mathrm{~m}) ; 1727(\mathrm{~m}) ; 1583(\mathrm{~m}) ; 1442(\mathrm{w}) ; 1370(\mathrm{w}) ; 1315(\mathrm{w}) ; 1262(\mathrm{w})$; $1104(\mathrm{~s}) ; 1012(\mathrm{~m}) ; 805(\mathrm{~m}) ; 757(\mathrm{~m}) ; 729(\mathrm{~m}) \mathrm{cm}^{-1}$.

MS (ESI): $m / z(\%): 863.47(100)[M+H]^{+}, 880.50(15)\left[M+\mathrm{NH}_{4}\right]^{+}, 282.28$ (3).

HRMS (ESI) $\mathrm{m} / \mathrm{z}$ : $[M+\mathrm{H}]^{+}$Calcd for $\mathrm{C}_{48} \mathrm{H}_{67} \mathrm{~N}_{2} \mathrm{O}_{12}$ : 863.4694; found 863.4758. 


\section{Disorazole C1 (1)}

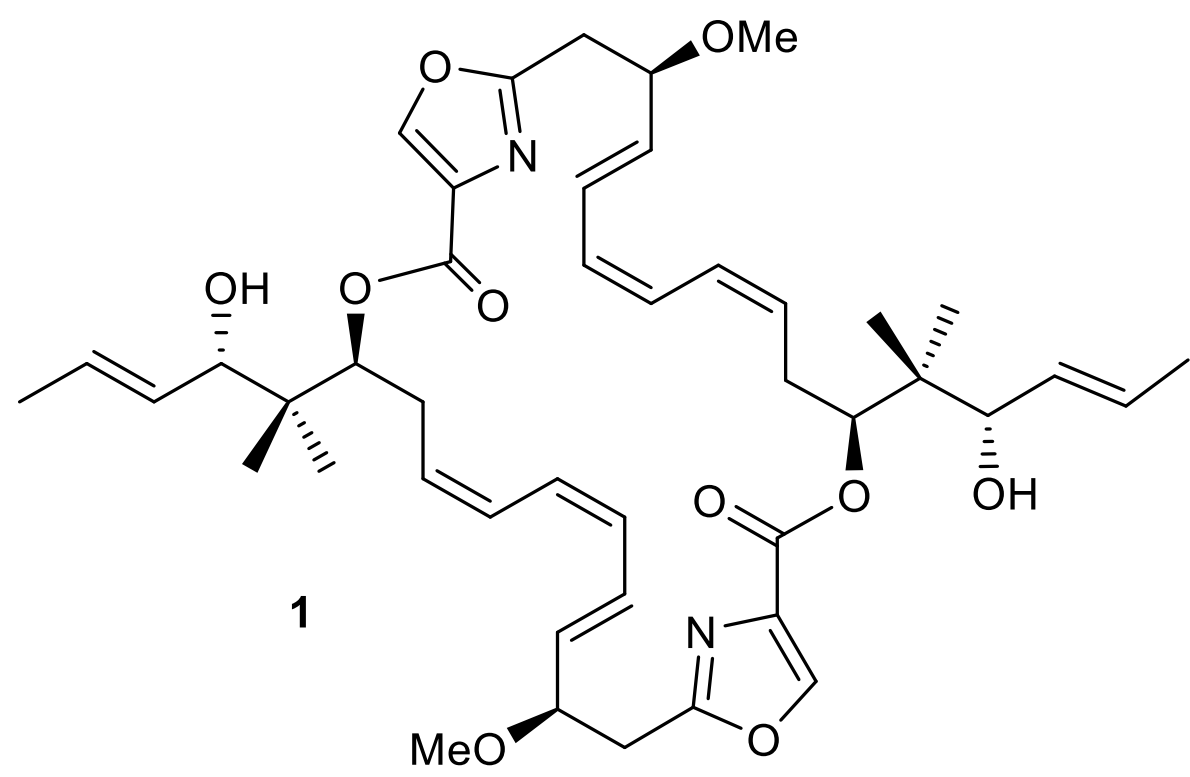

MOM protected disorazole $\mathrm{C}_{1} 30(22 \mathrm{mg}, 25.5 \mu \mathrm{mol})$ was dissolved in $\mathrm{CH}_{3} \mathrm{CN}(1.5 \mathrm{~mL})$ and cooled to $0^{\circ} \mathrm{C}$. 3 drops of $\mathrm{HBr}\left(48 \%\right.$ in $\left.\mathrm{H}_{2} \mathrm{O}\right)$ were slowly added and then the mixture was stirred for $1 \mathrm{~h}$ at $0^{\circ} \mathrm{C}$. The mixture was diluted with $\operatorname{EtOAc}(4 \mathrm{~mL})$ and washed with saturated aqueous $\mathrm{NaHCO}_{3}$ solution $(3 \mathrm{~mL})$. The aqueous phase was extracted with EtOAc (3x5 mL) and the organic extracts were dried over $\mathrm{Na}_{2} \mathrm{SO}_{4}$, filtered and concentrated in vacuo. The residue was purified by flash chromatography $\left(\mathrm{CH}_{2} \mathrm{Cl}_{2} / \mathrm{MeOH} 50: 1\right)$ to give Disorazole $\mathrm{C}_{1} 1$ (11 $\mathrm{mg}, 14.2 \mu \mathrm{mol}, 56 \%)$ as a colorless wax.

General Data: $\mathrm{C}_{44} \mathrm{H}_{58} \mathrm{~N}_{2} \mathrm{O}_{10}$; FW: 774.41; TLC: $\mathrm{R} f=0.20\left(\mathrm{CH}_{2} \mathrm{Cl}_{2} / \mathrm{MeOH} 50: 1\right)$; UV (+); Vanillin: dark green; $[\alpha]_{D}^{20}=-125.11(c=0.45, \mathrm{MeOH})$, lit. $[\alpha]_{D}^{22}=-124.8(c=0.6, \mathrm{MeOH})$.

${ }^{1} \mathrm{H}-\mathrm{NMR}\left(600 \mathrm{MHz}, \mathrm{CD}_{3} \mathrm{OD}\right): \delta(\mathrm{ppm}): 8.24(\mathrm{~s}, 2 \mathrm{H}, \mathrm{NC}=\mathrm{CH}) ; 6.50$ (dd, $J=15.1,11.3 \mathrm{~Hz}$, $\left.2 \mathrm{H}, \mathrm{CH}\left(\mathrm{OCH}_{3}\right) \mathrm{CH}=\mathrm{CH}\right) ; 6.40($ app t, $J=11.2 \mathrm{~Hz}, 2 \mathrm{H}, \mathrm{CH} 2 \mathrm{CH}=\mathrm{CH}) ; 6.28(\mathrm{dd}, J=11.3,11.1$ $\mathrm{Hz}, 2 \mathrm{H}$,

$\mathrm{CHCH}=\mathrm{CHCH}$ ); 5.91 (dd, $J=11.3,11.0 \mathrm{~Hz}, 2 \mathrm{H}, \mathrm{CHCH}=\mathrm{CHCH}$ ); 5.67 (dq, $J=15.2,6.2 \mathrm{~Hz}$, $2 \mathrm{H}, \mathrm{CH} 3 \mathrm{CH}=\mathrm{CH}$ ); 5.58 (ddd, $J=15.4,7.7,1.4 \mathrm{~Hz}, 2 \mathrm{H}, \mathrm{CH} 3 \mathrm{CH}=\mathrm{CH}$ ); 5.54 (dd, $J=15.1,8.4$ $\mathrm{Hz}, 2 \mathrm{H}, \mathrm{CH}(\mathrm{OCH} 3) \mathrm{CH}=\mathrm{CH}$ ); 5.48 (app dt, $J=10.2,6.4 \mathrm{~Hz}, 2 \mathrm{H}, \mathrm{CH} 2 \mathrm{CH}=\mathrm{CH}$ ); 5.26 (dd, $J=$ $11.3,2.2 \mathrm{~Hz}, 2 \mathrm{H}, \mathrm{CHOC}=0$ ); 4.13 (ddd, $J=7.9,7.2,5.3 \mathrm{~Hz}, 2 \mathrm{H}, \mathrm{CHOCH}$ ); 3.84 (d, $J=7.5$ $\mathrm{Hz}, 2 \mathrm{H}, \mathrm{CHOH}$ ); 3.21 (s, $6 \mathrm{H}, \mathrm{CHOCH}_{3}$ ); 3.00 (dd, $J=15.4,7.4 \mathrm{~Hz}, 2 \mathrm{H}, \mathrm{CH}_{2} \mathrm{CHOCH}_{3}$ ); 2.77 (dd, $J=15.4,7.2 \mathrm{~Hz}, 2 \mathrm{H}, \mathrm{CH}_{2} \mathrm{CHOCH}_{3}$ ); 2.69 (ddd, $J=13.8,10.9,10.2 \mathrm{~Hz}, 2 \mathrm{H}, \mathrm{CH}_{2} \mathrm{CH}=\mathrm{CH}$ ); 2.39 (dd, $J=13.6,6.2 \mathrm{~Hz}, 2 \mathrm{H}, \mathrm{CH}_{2} \mathrm{CH}=\mathrm{CH}$ ); 1.70 (dd, $J=6.3,1.1 \mathrm{~Hz}, 6 \mathrm{H}, \mathrm{CH}=\mathrm{CHCH}_{3}$ ); $1.01\left(\mathrm{~s}, 6 \mathrm{H}, \mathrm{CCH}_{3}\right) ; 0.954\left(\mathrm{~s}, 6 \mathrm{H}, \mathrm{CCH}_{3}\right)$.

${ }^{13} \mathrm{C}$-NMR (151 MHz, CD 3 OD): $\delta(\mathrm{ppm}): 164.1(\mathrm{C}=\mathrm{O}) ; 162.2(\mathrm{C}=\mathrm{N}) ; 145.8(\mathrm{CH}) ; 134.2(\mathrm{C}) ;$ $134.1(\mathrm{CH}) ; 131.7(\mathrm{CH}) ; 130.9(\mathrm{CH}) ; 130.0(\mathrm{CH}) ; 129.6(\mathrm{CH}) ; 129.3(\mathrm{CH}) ; 127.4(\mathrm{CH}) ; 126.8$ $(\mathrm{CH}) ; 80.6(\mathrm{CH}) ; 78.7(\mathrm{CH}) ; 77.8(\mathrm{CH}) ; 56.8\left(\mathrm{CH}_{3}\right) ; 42.7(\mathrm{C}) ; 36.0\left(\mathrm{CH}_{2}\right) ; 29.2\left(\mathrm{CH}_{2}\right) ; 19.4$ $\left(\mathrm{CH}_{3}\right) ; 19.3\left(\mathrm{CH}_{3}\right) ; 18.1\left(\mathrm{CH}_{3}\right)$.

IR(neat): 3419 (br); 2924 (m); 2854 (m); 1723 (m); 1583 (m); 1447 (w); 1367 (w); 1312 (w); $1261(\mathrm{w}) ; 1101(\mathrm{~s}) ; 1010(\mathrm{~m}) ; 803(\mathrm{~m}) ; 758(\mathrm{~m}) ; 729(\mathrm{~m}) \mathrm{cm}^{-1}$.

MS (ESI): $m / z(\%): 775.41(100)[M+H]^{+}, 792.44(28)\left[M+N_{4}\right]^{+}, 797.40(6) ; 757.40(5)$.

HRMS (ESI) $\mathrm{m} / \mathrm{z}$ : $[\mathrm{M}+\mathrm{H}]^{+}$Calcd for $\mathrm{C}_{44} \mathrm{H}_{59} \mathrm{~N}_{2} \mathrm{O}_{10}$ : 775.4170 ; found 775.4198 . 
Results and Discussion

Table 1. ${ }^{1} \mathrm{H}$ NMR Comparison for Disorazole $\mathrm{C}_{1}$

\begin{tabular}{|c|c|c|c|c|c|c|c|}
\hline & \multicolumn{3}{|c|}{ This work } & \multicolumn{3}{|c|}{ Wipf } & \\
\hline $\begin{array}{c}\text { NMR } \\
\text { field } \\
{[\mathrm{M} \mathrm{Hz}]}\end{array}$ & \multicolumn{3}{|c|}{600} & \multicolumn{3}{|c|}{600} & \\
\hline Solvent & \multicolumn{3}{|c|}{$\mathrm{CD}_{3} \mathrm{OD}_{3}$} & \multicolumn{3}{|c|}{$\mathrm{CD}_{3} \mathrm{OD}_{3}$} & \\
\hline Proton & $\delta[p p m]$ & Multiplicity & $J[\mathrm{~Hz}]$ & $\delta[p p m]$ & Multiplicity & $J[\mathrm{~Hz}]$ & $\Delta \delta$ \\
\hline $3-\mathrm{H}$ & 8.24 & $s$ & - & 8.23 & $s$ & - & +0.01 \\
\hline $5-\mathrm{Ha}$ & 3.00 & dd & $15.4,7.4$ & 2.99 & dd & $15.5,7.3$ & +0.01 \\
\hline $5-\mathrm{Hb}$ & 2.77 & dd & $15.4,5.2$ & 2.76 & dd & $15.6,5.0$ & +0.01 \\
\hline $6-\mathrm{H}$ & 4.13 & ddd & $7.9,7.2,5.3$ & 4.13 & ddd & $7.9,7.2,5.1$ & - \\
\hline $7-\mathrm{H}$ & 5.54 & dd & $15.1,8.4$ & 5.54 & dd & $15.1,8.3$ & - \\
\hline $8-\mathrm{H}$ & 6.50 & dd & $15.1,11.3$ & 6.50 & dd & $14.9,11.3$ & - \\
\hline $9-\mathrm{H}$ & 5.91 & dd & $11.3,11.0$ & 5.91 & dd & $11.3,11.0$ & - \\
\hline $10-\mathrm{H}$ & 6.28 & dd & $11.3,11.1$ & 6.28 & dd & $11.3,11.1$ & - \\
\hline $11-\mathrm{H}$ & 6.40 & app t & 11.2 & 6.40 & app t & 11.2 & - \\
\hline $12-\mathrm{H}$ & 5.48 & app dt & $10.2,6.4$ & 5.48 & app dt & $10.0,6.7$ & - \\
\hline $13-\mathrm{Ha}$ & 2.69 & ddd & $13.8,10.9,10.2$ & 2.69 & ddd & $13.8,10.9,10.1$ & - \\
\hline $13-\mathrm{Hb}$ & 2.39 & dd & $13.6,6.2$ & 2.38 & dd & $13.1,6.1$ & +0.01 \\
\hline $14-\mathrm{H}$ & 5.26 & dd & $11.3,2.2$ & 5.25 & dd & $11.3,2.1$ & +0.01 \\
\hline $16-\mathrm{H}$ & 3.84 & $d$ & 7.5 & 3.84 & $d$ & 7.3 & - \\
\hline $17-\mathrm{H}$ & 5.58 & ddd & $15.4,7.7,1.4$ & 5.57 & ddd & $15.4,7.6,1.5$ & +0.01 \\
\hline $18-\mathrm{H}$ & 5.67 & $\mathrm{dq}$ & $15.2,6.2$ & 5.66 & dq & $15.1,6.3$ & +0.01 \\
\hline $19-\mathrm{H} 3$ & 1.70 & dd & $6.3,1.1$ & 1.69 & dd & $6.1,1.1$ & +0.01 \\
\hline $20-\mathrm{H} 3$ & 1.01 & $s$ & - & 1.00 & $s$ & - & +0.01 \\
\hline $21-\mathrm{H} 3$ & 0.95 & s & - & 0.95 & $\mathrm{~s}$ & - & - \\
\hline $6-\mathrm{OCH}_{3}$ & 3.21 & $s$ & - & 3.21 & $s$ & - & - \\
\hline $16-\mathrm{OH}$ & - & - & - & - & - & - & - \\
\hline $16-\mathrm{OH}$ & - & - & - & - & - & - & - \\
\hline
\end{tabular}


Table 2. ${ }^{13} \mathrm{C}$ NMR Comparison for Disorazole $\mathrm{C}_{1}$

\begin{tabular}{|c|c|c|c|c|c|}
\hline & This work & Wipf & Hoyveda & Isolation & This work-Wipf \\
\hline Carbon & $\delta[p p m]$ & $\delta$ [ppm] & $\delta$ [ppm] & $\delta$ [ppm] & $\Delta \delta$ \\
\hline 1 & 162.24 & 162.26 & 162.26 & 162.27 & -0.02 \\
\hline 2 & 134.07 & 134.09 & 134.08 & 134.12 & -0.02 \\
\hline 3 & 145.83 & 145.83 & 145.84 & 145.81 & - \\
\hline 4 & 164.11 & 164.12 & 164.13 & 164.13 & -0.01 \\
\hline 5 & 35.98 & 35.97 & 35.98 & 35.97 & +0.01 \\
\hline 6 & 80.56 & 80.57 & 80.56 & 80.59 & -0.01 \\
\hline 7 & 134.15 & 134.15 & 134.15 & 134.17 & - \\
\hline 8 & 129.96 & 129.96 & 129.98 & 129.94 & - \\
\hline 9 & 129.29 & 129.30 & 129.30 & 129.31 & -0.01 \\
\hline 10 & 126.80 & 126.79 & 126.80 & 126.79 & +0.01 \\
\hline 11 & 127.36 & 127.36 & 127.37 & 127.35 & - \\
\hline 12 & 130.88 & 130.88 & 130.89 & 130.88 & - \\
\hline 13 & 29.24 & 29.24 & 29.24 & 29.27 & - \\
\hline 14 & 78.73 & 78.75 & 78.75 & 78.81 & -0.02 \\
\hline 15 & 42.70 & 42.70 & 42.70 & 42.73 & - \\
\hline 16 & 77.83 & 77.84 & 77.84 & 77.85 & -0.01 \\
\hline 17 & 131.67 & 131.68 & 131.67 & 131.70 & -0.01 \\
\hline 18 & 129.63 & 129.63 & 129.64 & 129.62 & - \\
\hline 19 & 18.05 & 18.03 & 18.04 & 18.02 & +0.02 \\
\hline 20 & 19.41 & 19.41 & 19.42 & 19.45 & - \\
\hline 21 & 19.32 & 19.32 & 19.35 & 19.34 & - \\
\hline $6-\mathrm{OCH}_{3}$ & 56.83 & 56.83 & 56.83 & 56.84 & - \\
\hline
\end{tabular}

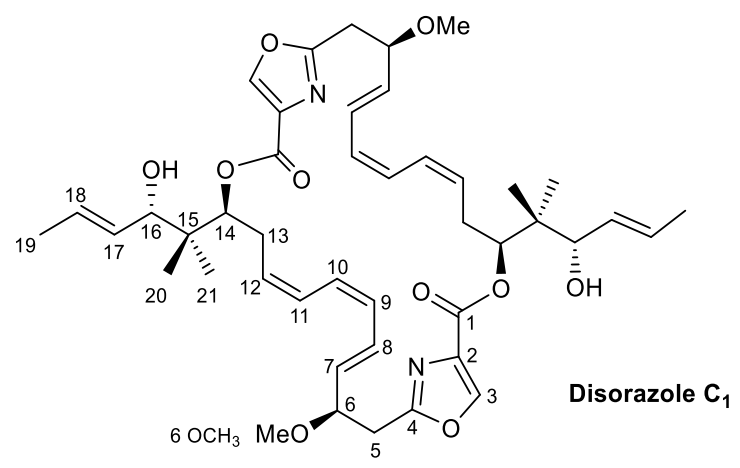




\section{References}

[1] a) Braun M., Waldmüller D. Simple Three-Step Synthesis of $(R)$ and (S)-4-Amino-3hydroxybutanoic Acid (GABOB) by Stereoselective Aldol Addition. Synthesis 1989, 856858; b) Braun M., Gräf S., Herzo S. (R)-(+)-2-HYROXY-1,2,2-TRIPHENYL ACETATE. Org. Synth. 1995, 72, 32.

[2] Schinzer D., Bauer A., Böhm O. M., Limberg A., Cordes M. Total Synthesis of (-)Epothilone A. Chem. Eur. J. 1999, 5(9), 2483.

[3] Kubota K., Leighton J. L. A Highly Practical and Enantioselective Reagent for the Allylation of Aldehydes. Angew. Chem., Int. Ed. 2003, 42, 946.

[4] Marshall J. A., Salovich J. M., Shearer B. G. Stereoselective synthesis of a nonracemic hydronaphtalene subunit of kijanolide. J. Org. Chem. 1990, 55, 8, 2398-2403. 
${ }^{1} \mathrm{H}$ NMR $\left(600 \mathrm{MHz}, \mathrm{CDCl}_{3}\right)$

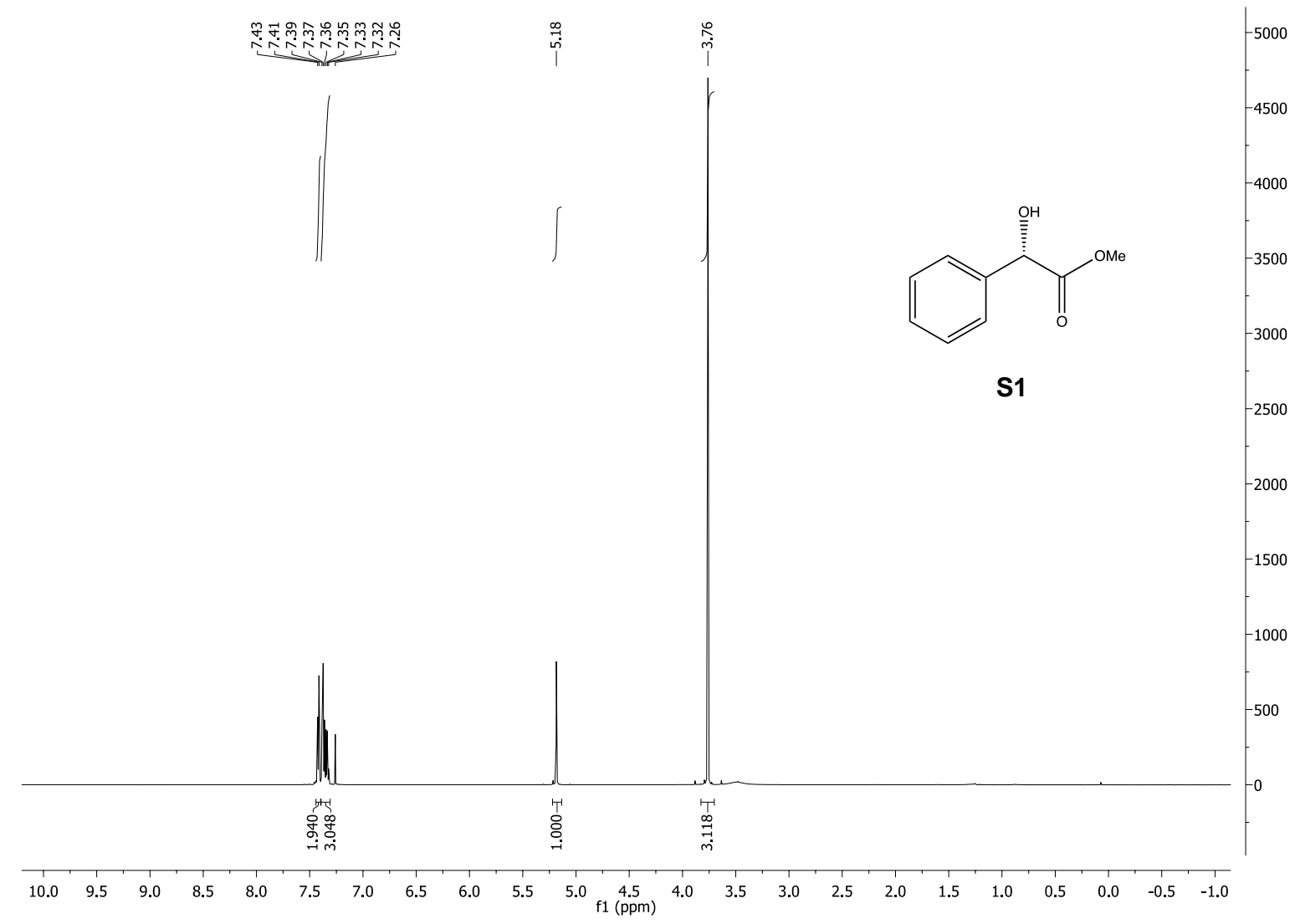

${ }^{13} \mathrm{C}$ NMR (151 MHz, CDCl 3 )

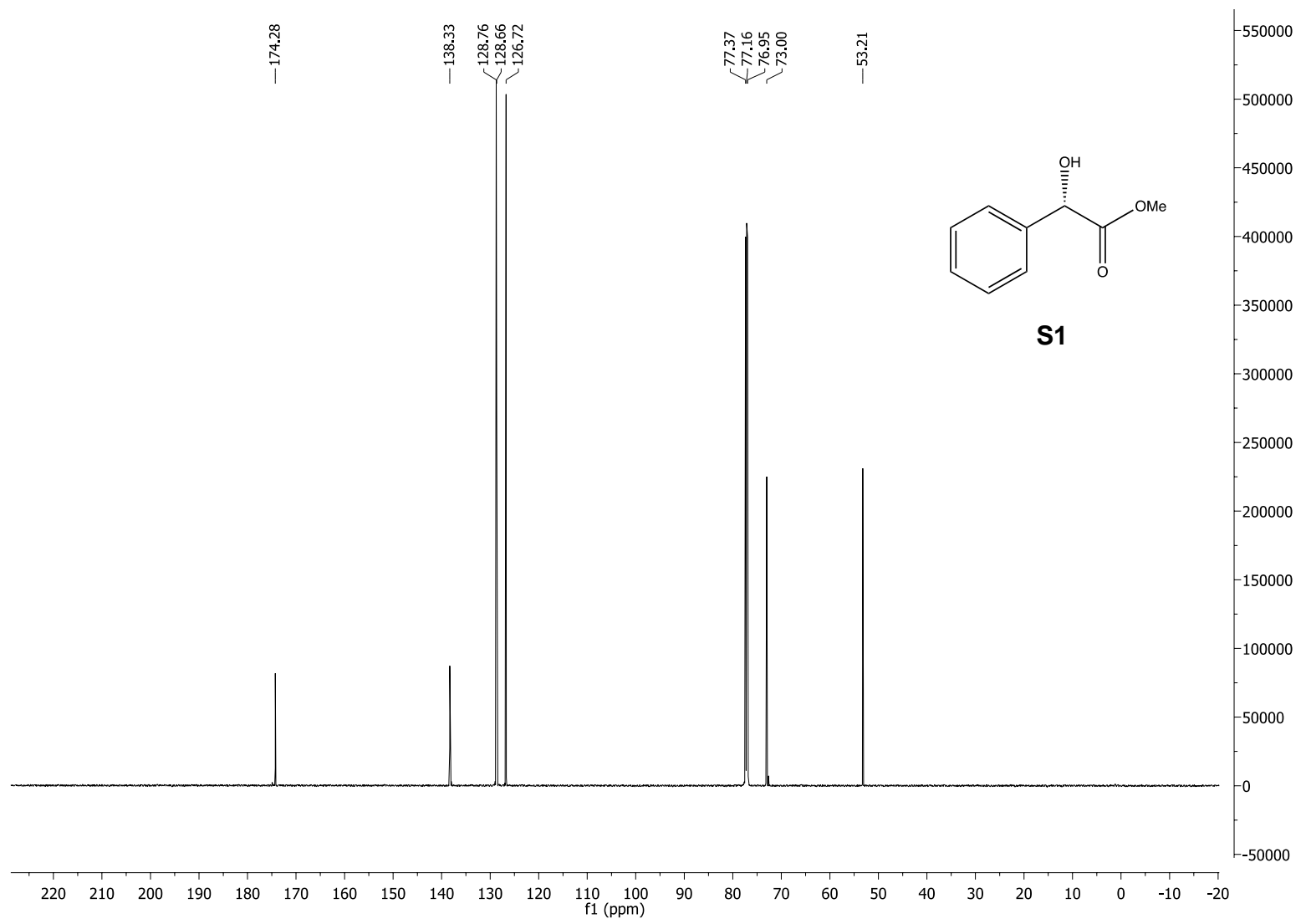


${ }^{1} \mathrm{H}$ NMR (400 MHz, $\mathrm{CDCl}_{3}$ )

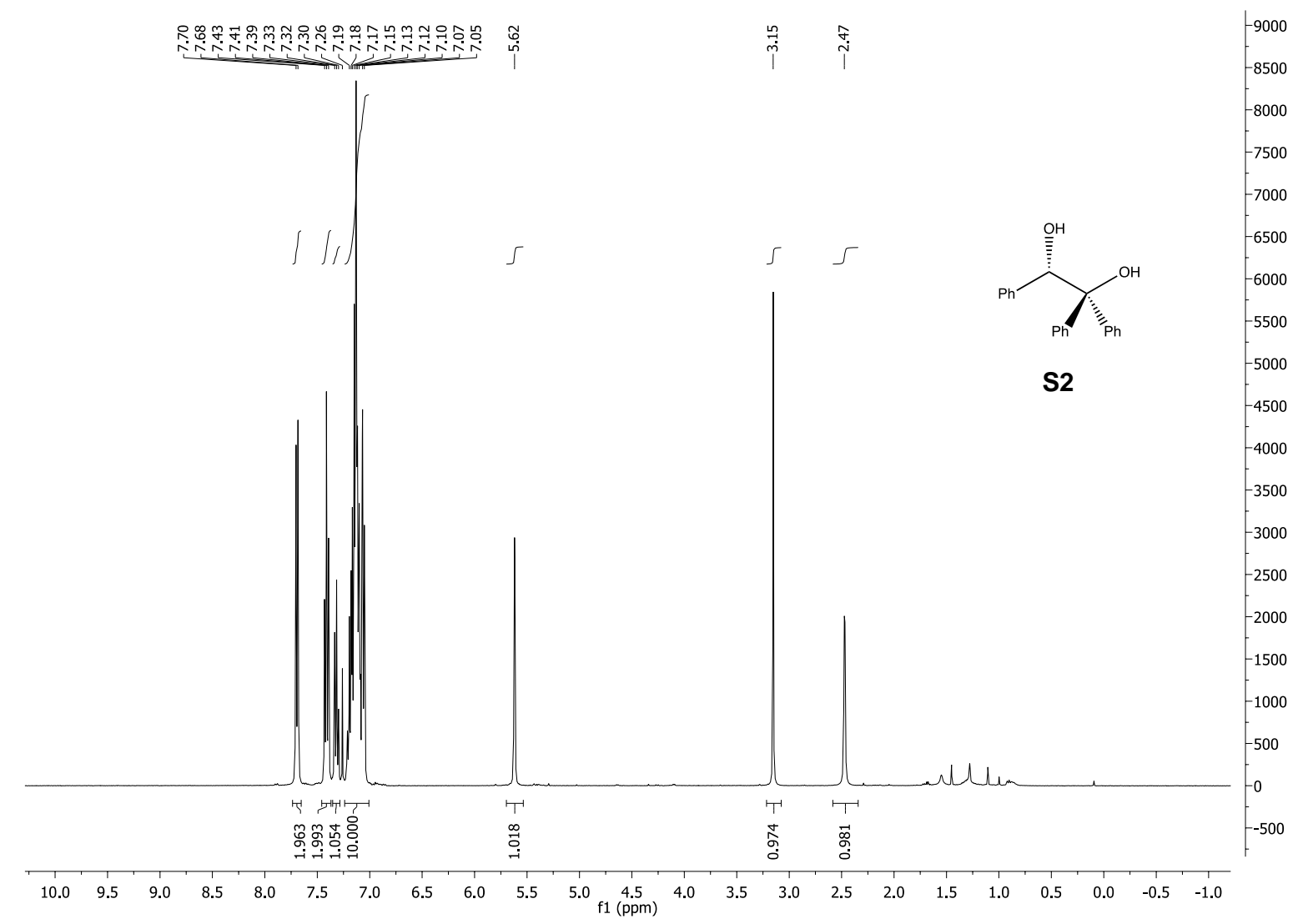

${ }^{13} \mathrm{C}$ NMR $\left(100 \mathrm{MHz}, \mathrm{CDCl}_{3}\right)$

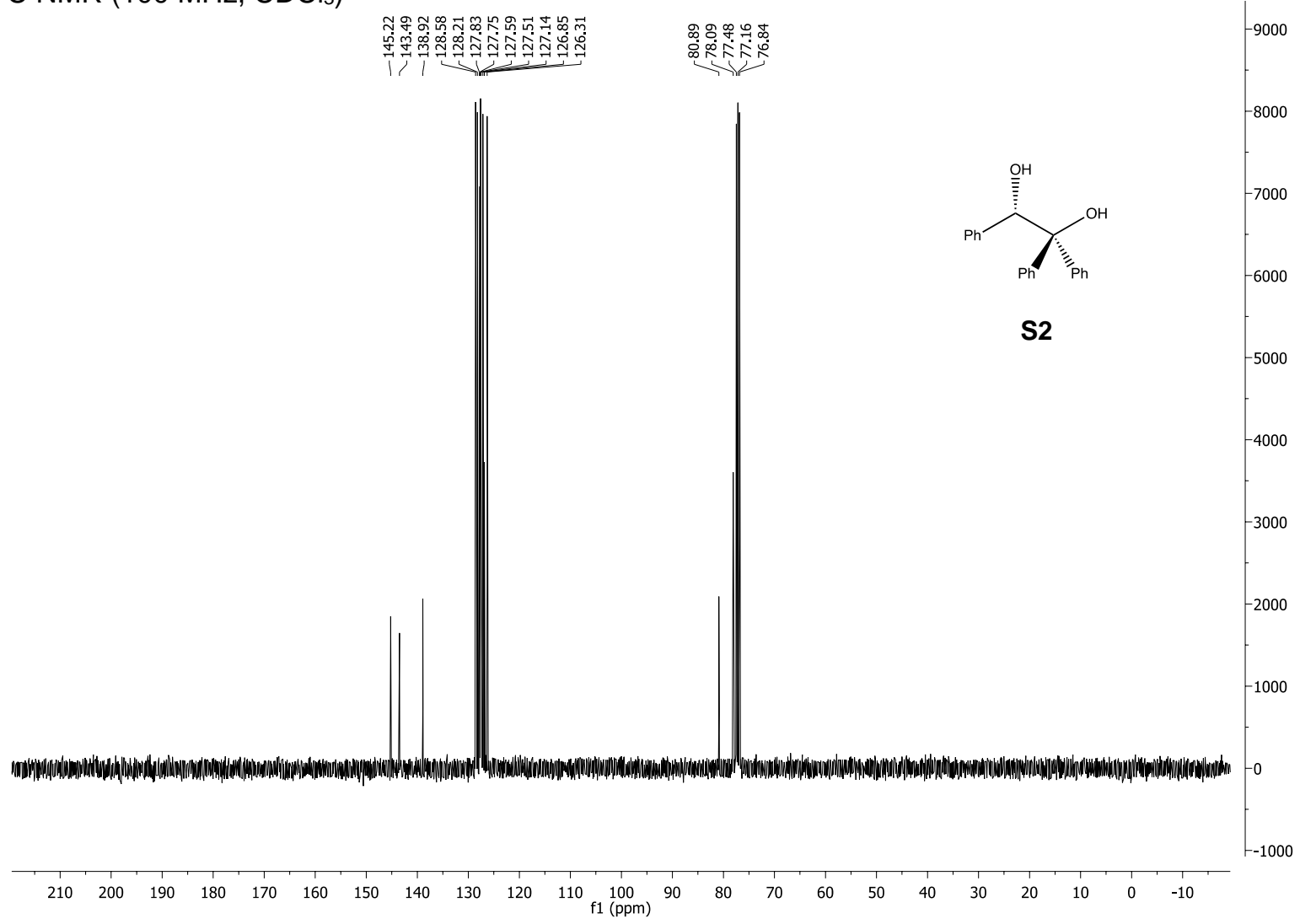


${ }^{1} \mathrm{H}$ NMR $\left(600 \mathrm{MHz}, \mathrm{CDCl}_{3}\right)$

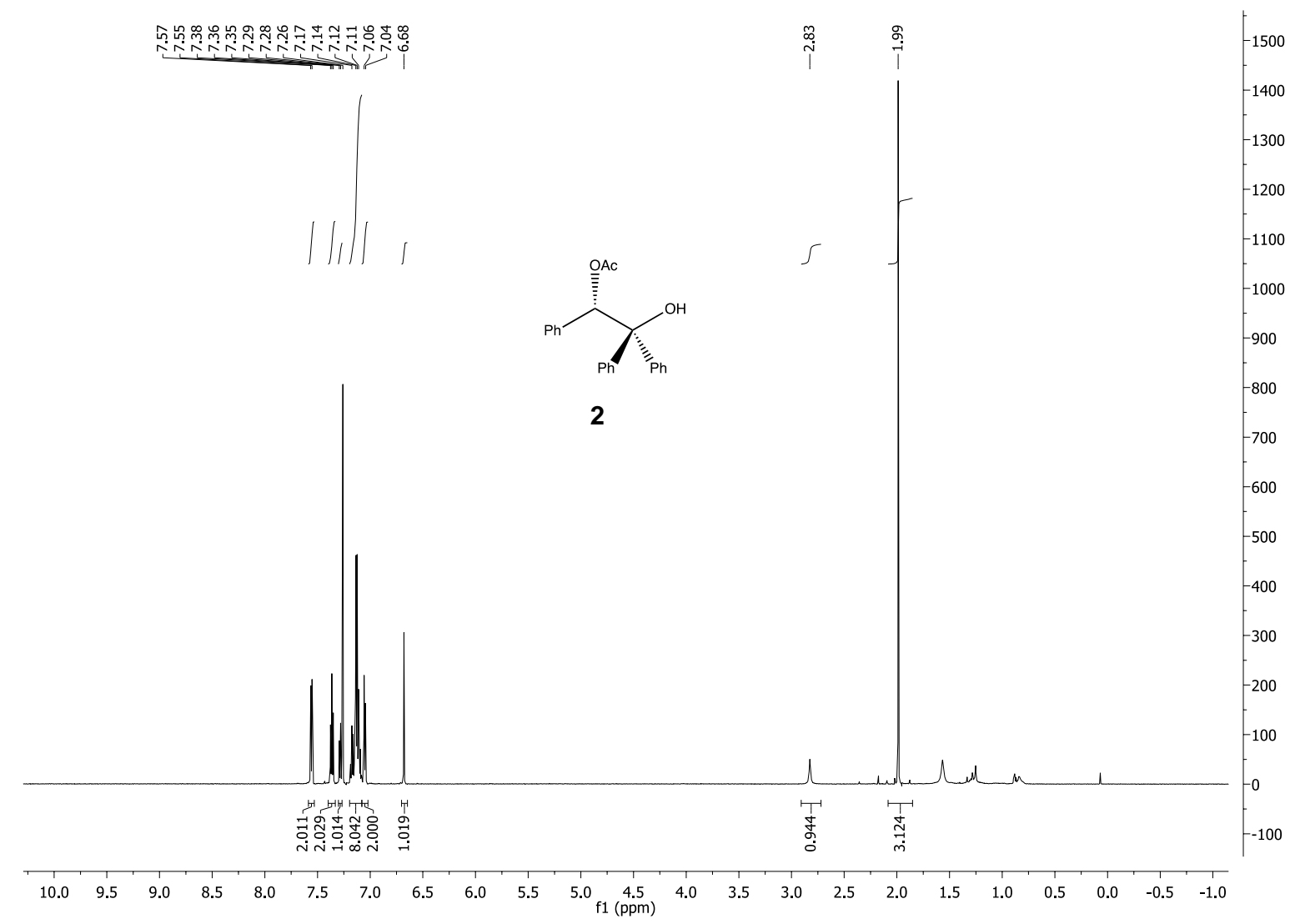

${ }^{13} \mathrm{C}$ NMR (151 MHz, $\mathrm{CDCl}_{3}$ )

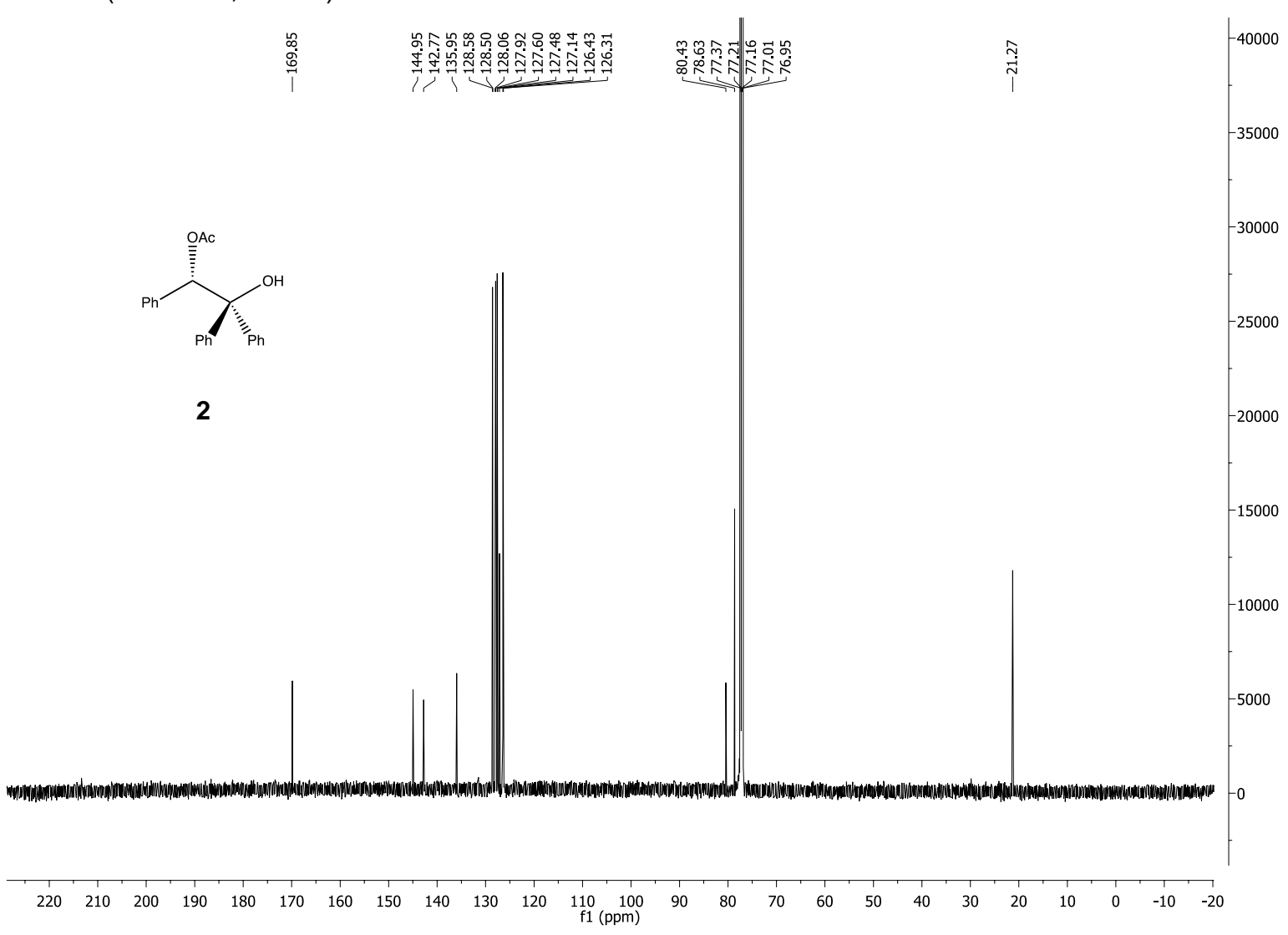


${ }^{1} \mathrm{H}$ NMR $\left(600 \mathrm{MHz}, \mathrm{CDCl}_{3}\right)$

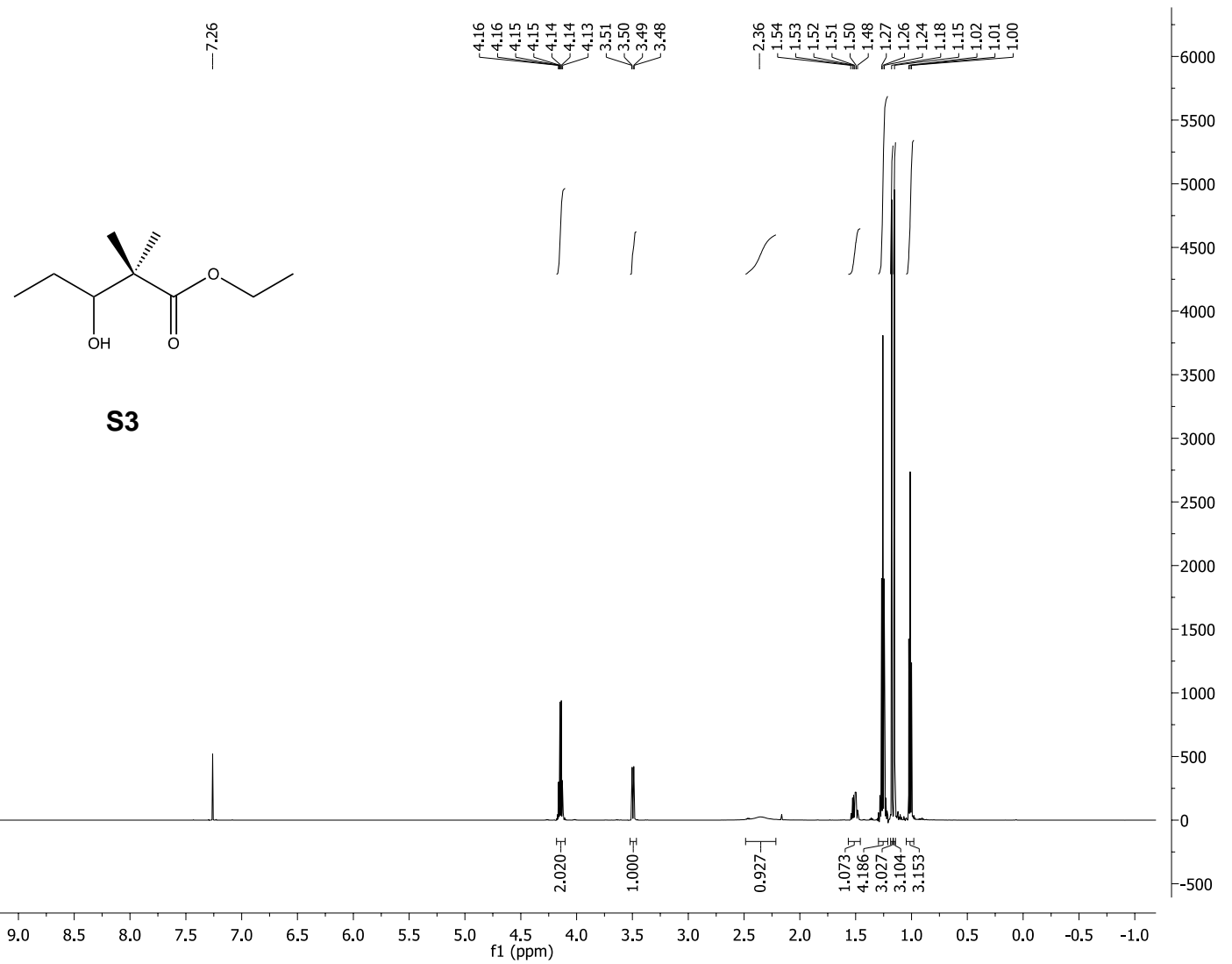

${ }^{13} \mathrm{C} \mathrm{NMR}\left(151 \mathrm{MHz}, \mathrm{CDCl}_{3}\right)$

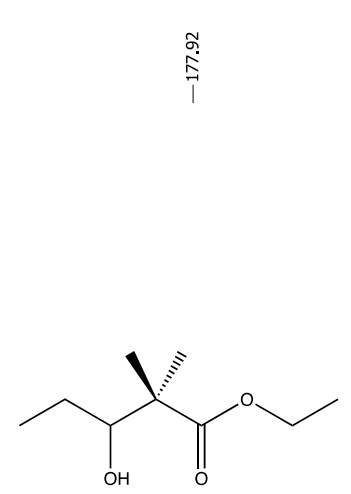

S3

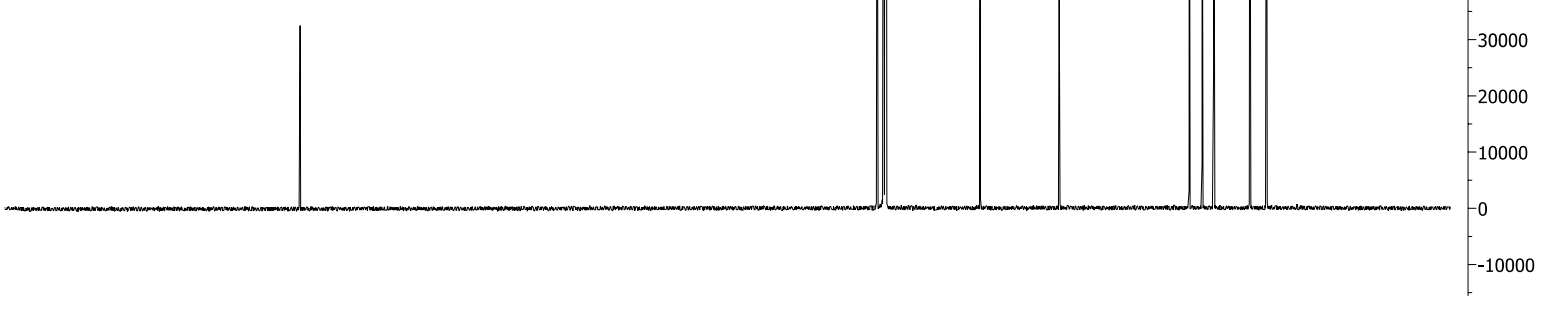

$\begin{array}{rllllllllllllllllllllllllllllll}220 & 210 & 200 & 190 & 180 & 170 & 160 & 150 & 140 & 130 & 120 & 110 & 100 & 90 & 80 & 70 & 60 & 50 & 40 & 30 & 20 & 10 & 0 & -10 & -20\end{array}$ 
${ }^{1} \mathrm{H}$ NMR $\left(600 \mathrm{MHz}, \mathrm{CDCl}_{3}\right)$

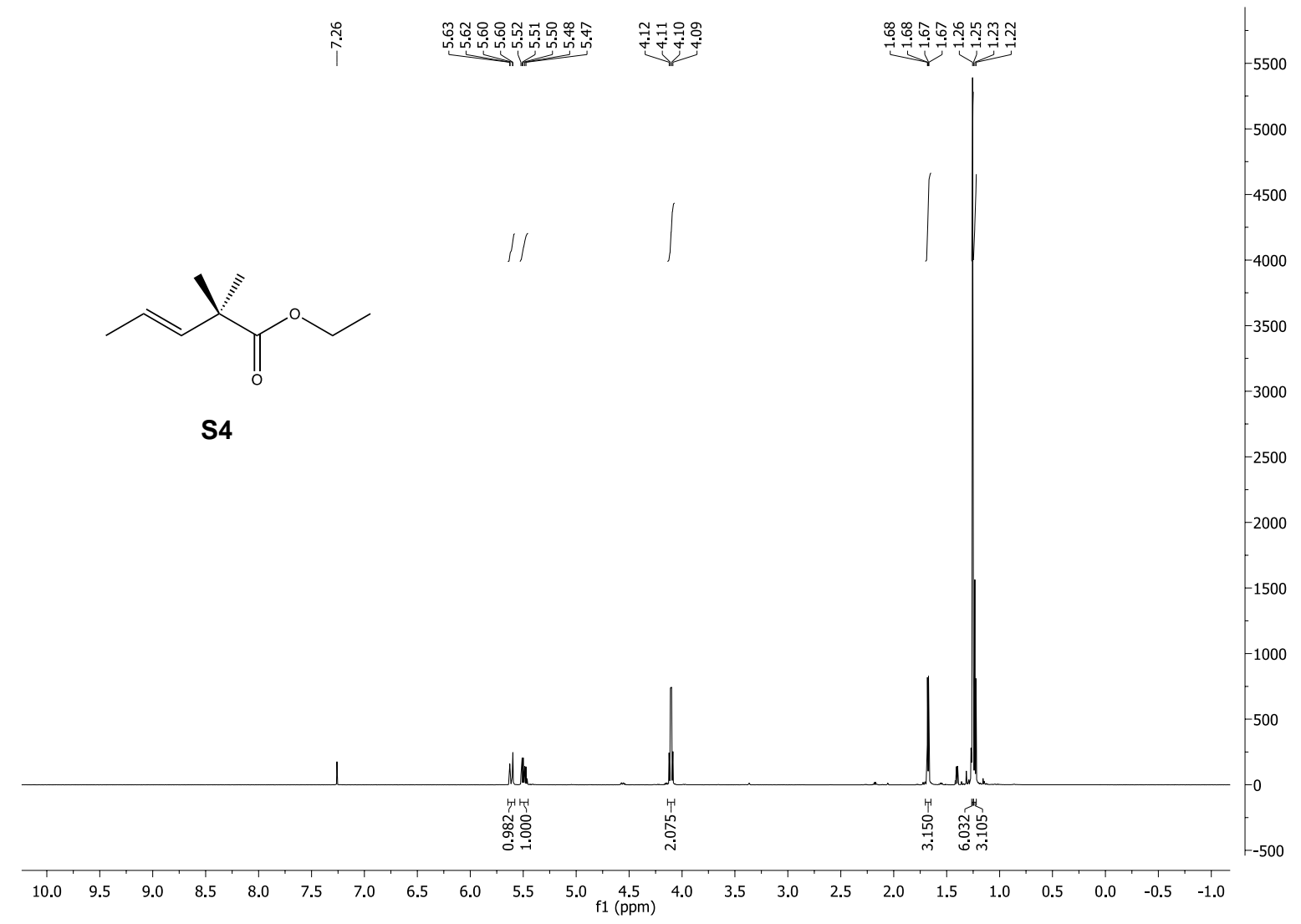

$\left.{ }^{13} \mathrm{C} \mathrm{NMR} \mathrm{(151} \mathrm{MHz,} \mathrm{CDCl}_{3}\right)$

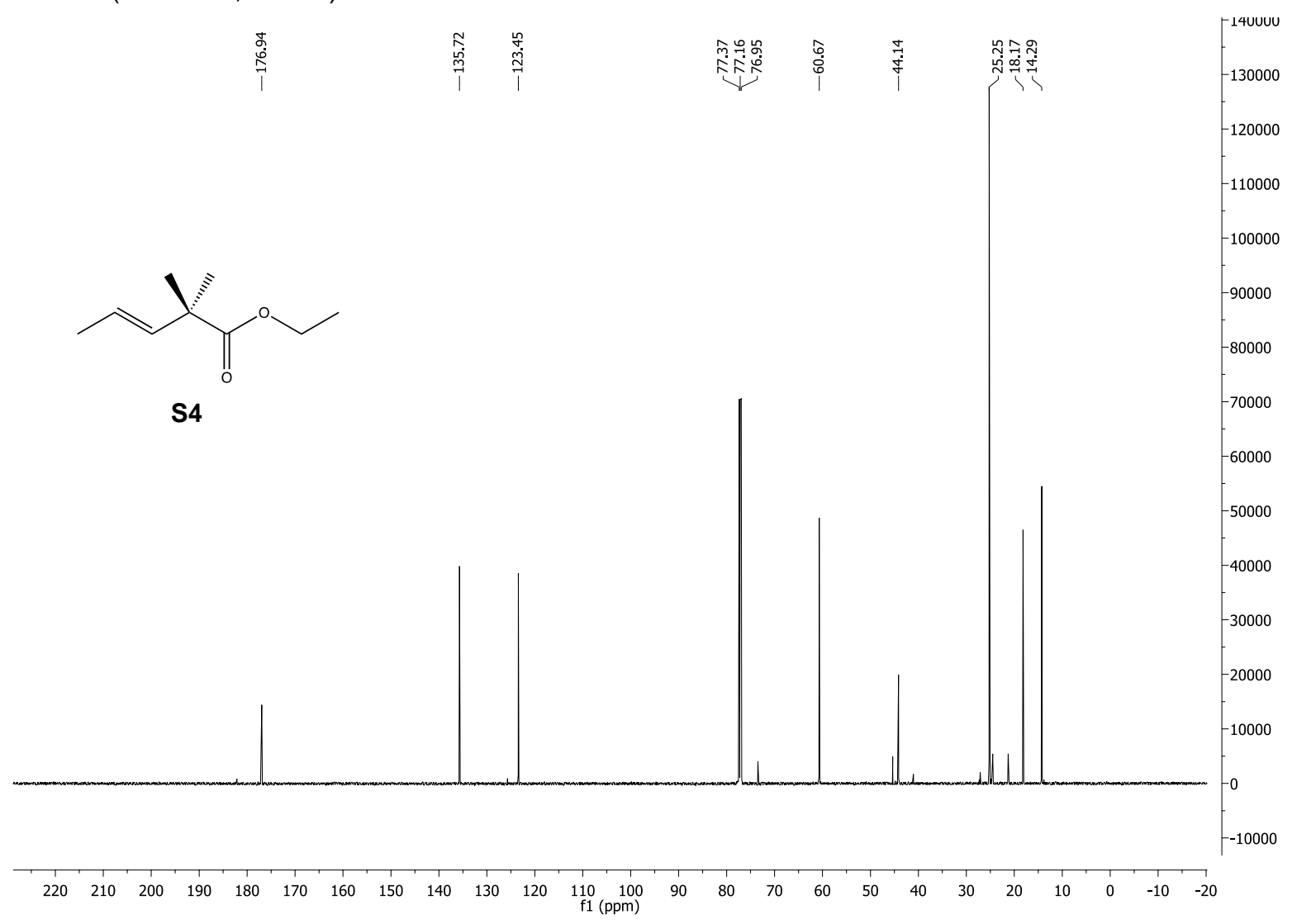


${ }^{1} \mathrm{H} \mathrm{NMR}\left(400 \mathrm{MHz}, \mathrm{CDCl}_{3}\right)$

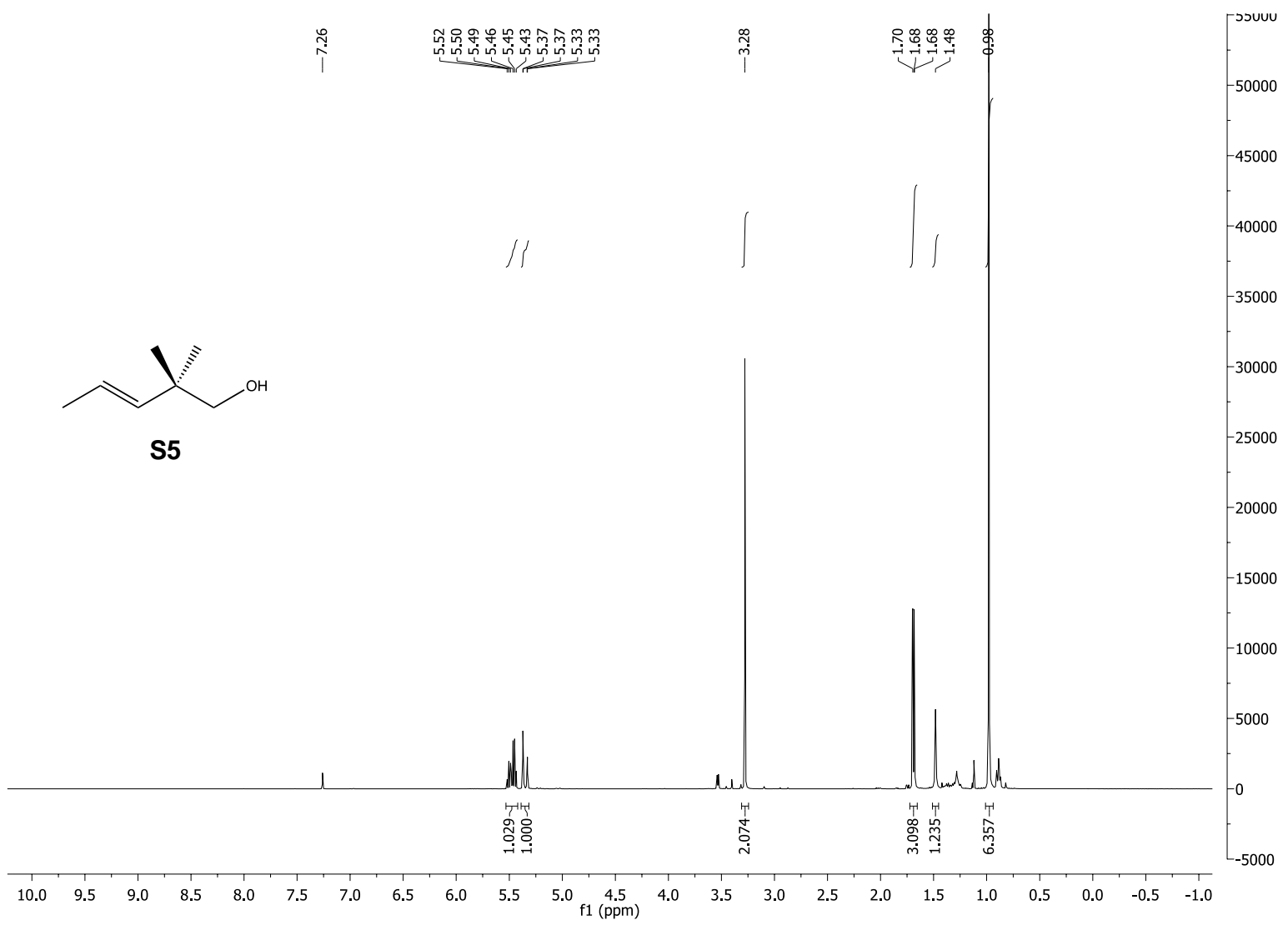

${ }^{13} \mathrm{C} \mathrm{NMR}\left(100 \mathrm{MHz}, \mathrm{CDCl}_{3}\right)$

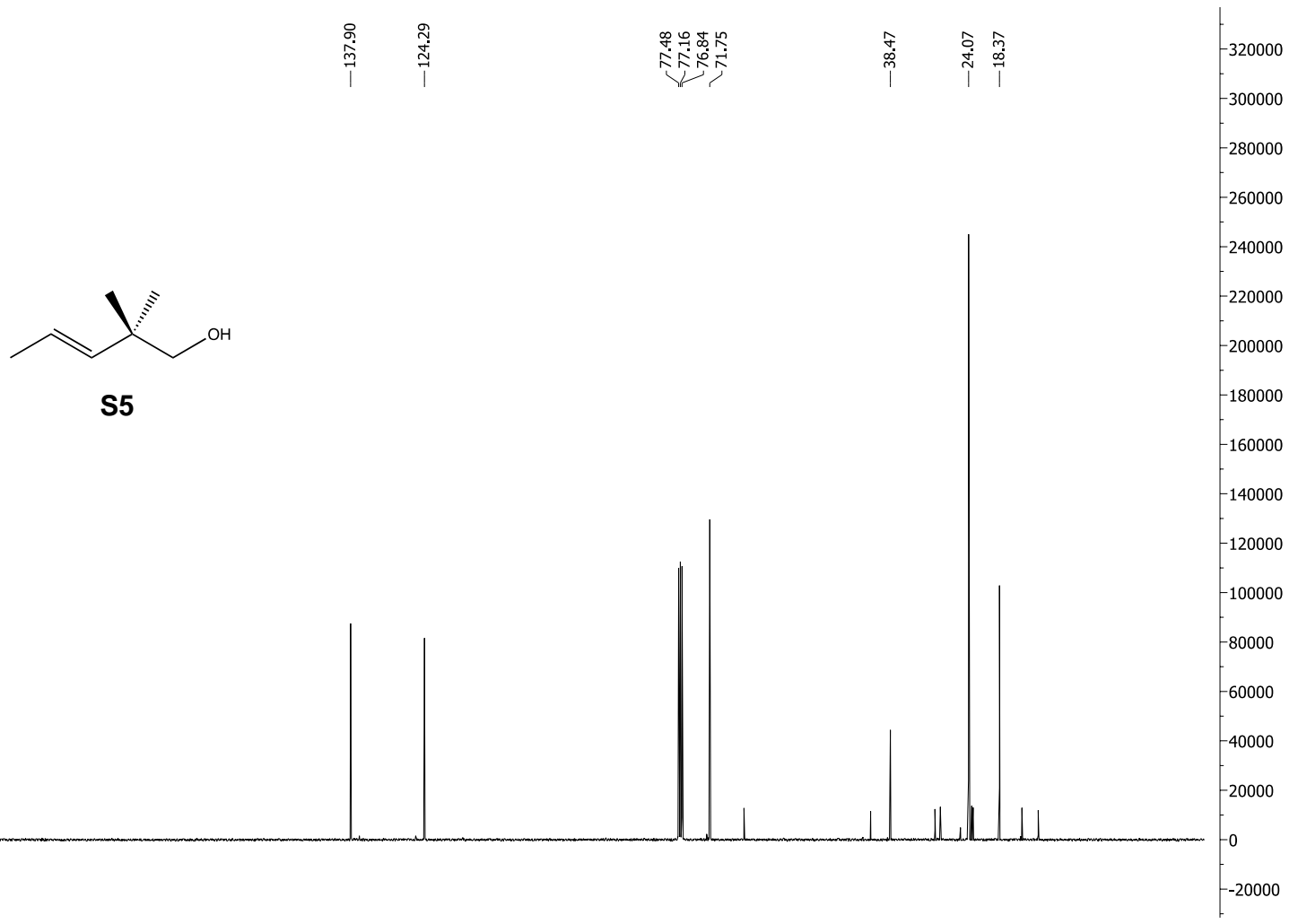

$\begin{array}{lllllllllllllllllllllllllllll}210 & 200 & 190 & 180 & 170 & 160 & 150 & 140 & 130 & 120 & 110 & 100 & 90 & 80 & 70 & 60 & 50 & 40 & 30 & 20 & 10 & 0 & -10\end{array}$ 
${ }^{1} \mathrm{H}$ NMR $\left(600 \mathrm{MHz}, \mathrm{CDCl}_{3}\right)$

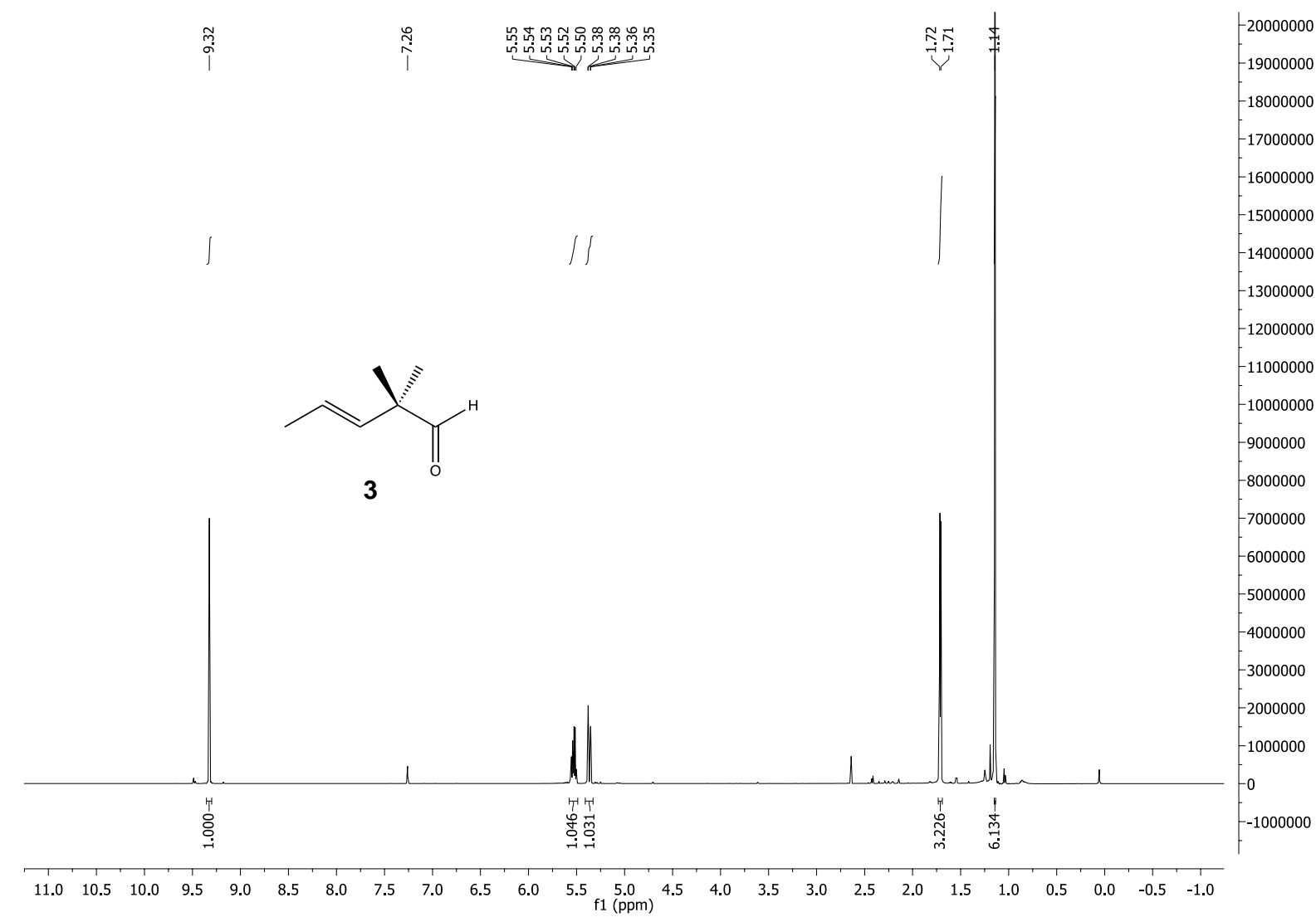

${ }^{13} \mathrm{C}$ NMR (151 MHz, $\left.\mathrm{CDCl}_{3}\right)$

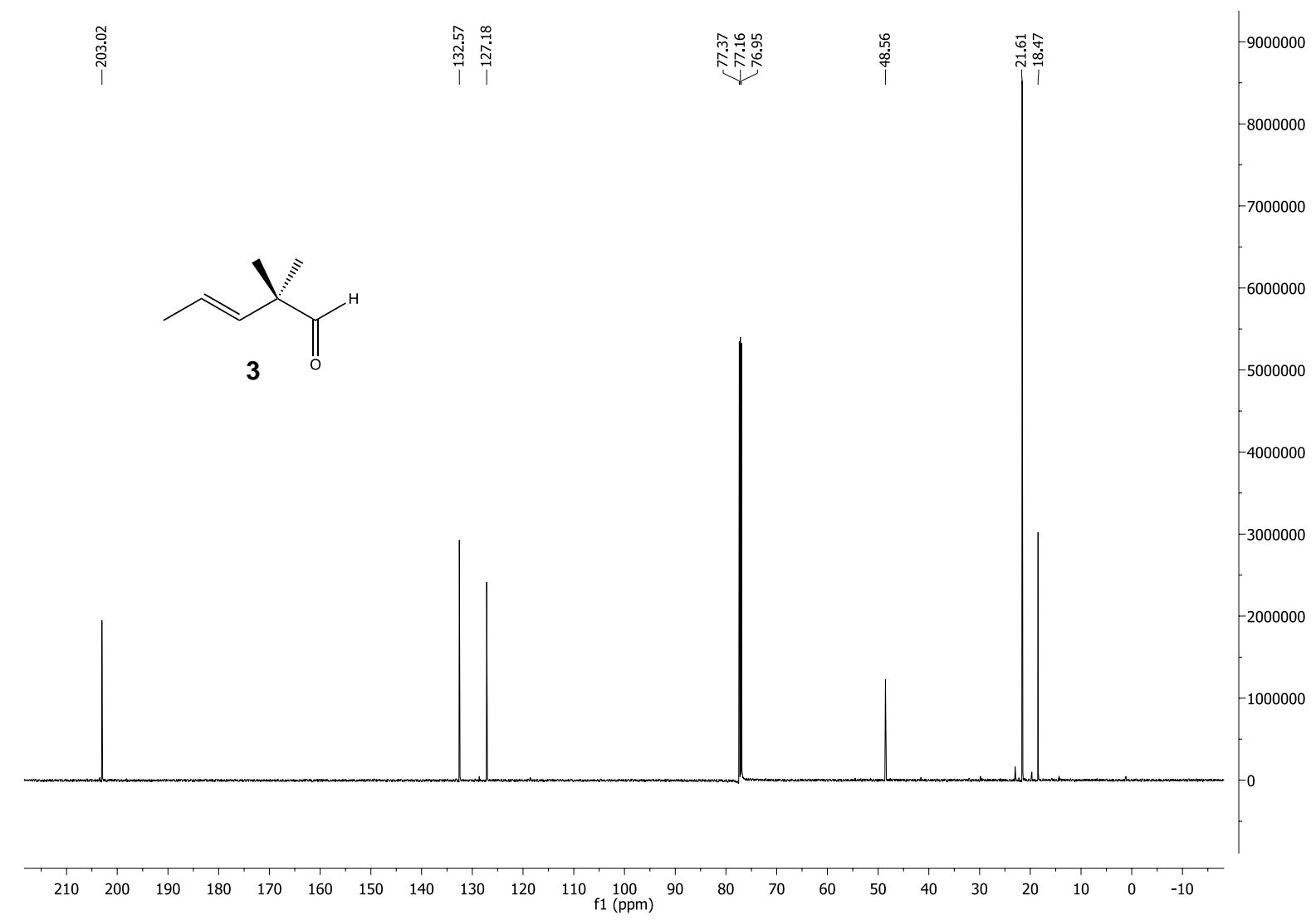


${ }^{1} \mathrm{H} \mathrm{NMR}\left(400 \mathrm{MHz}, \mathrm{CDCl}_{3}\right)$

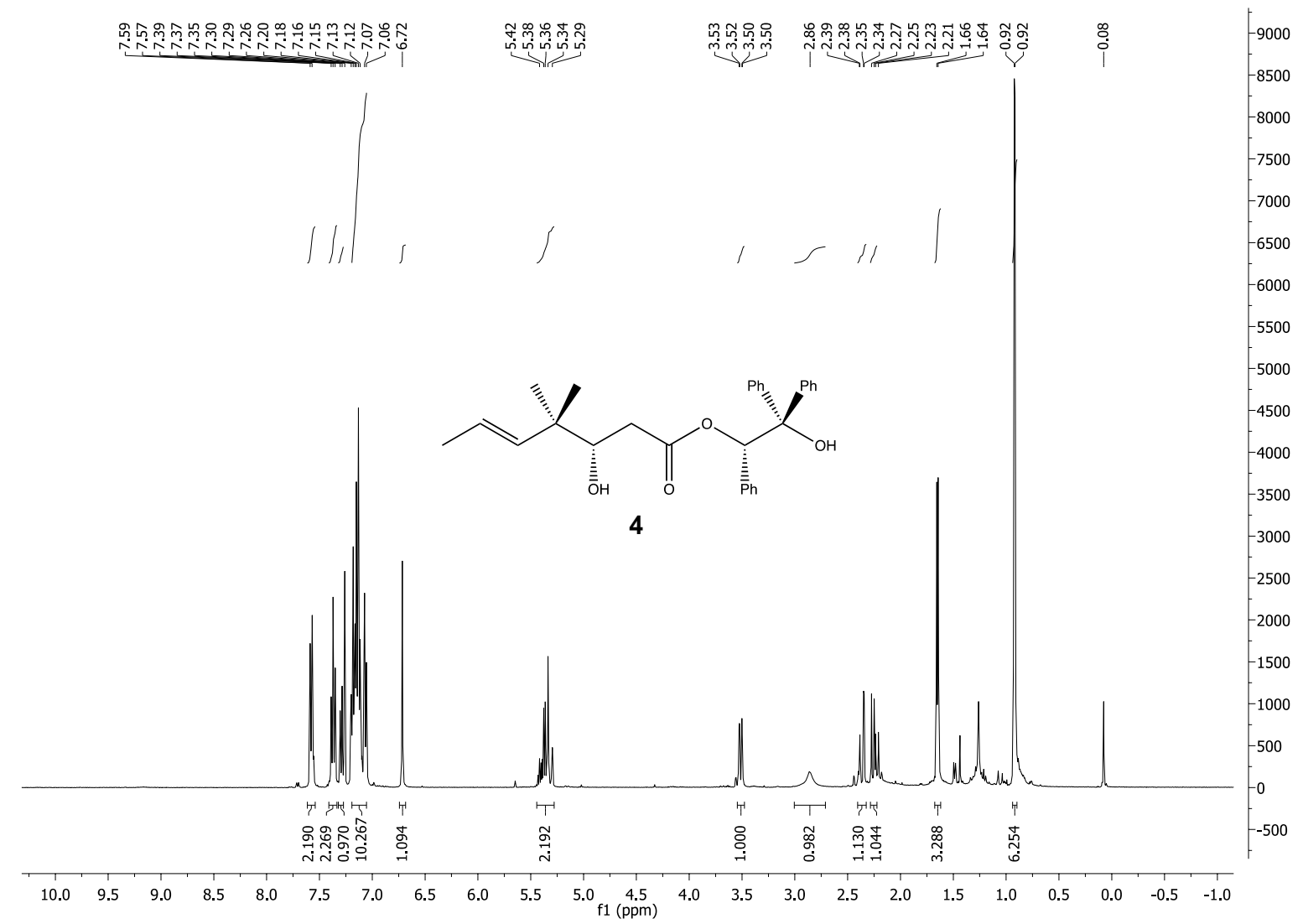

${ }^{13} \mathrm{C}$ NMR $\left(100 \mathrm{MHz}, \mathrm{CDCl}_{3}\right)$

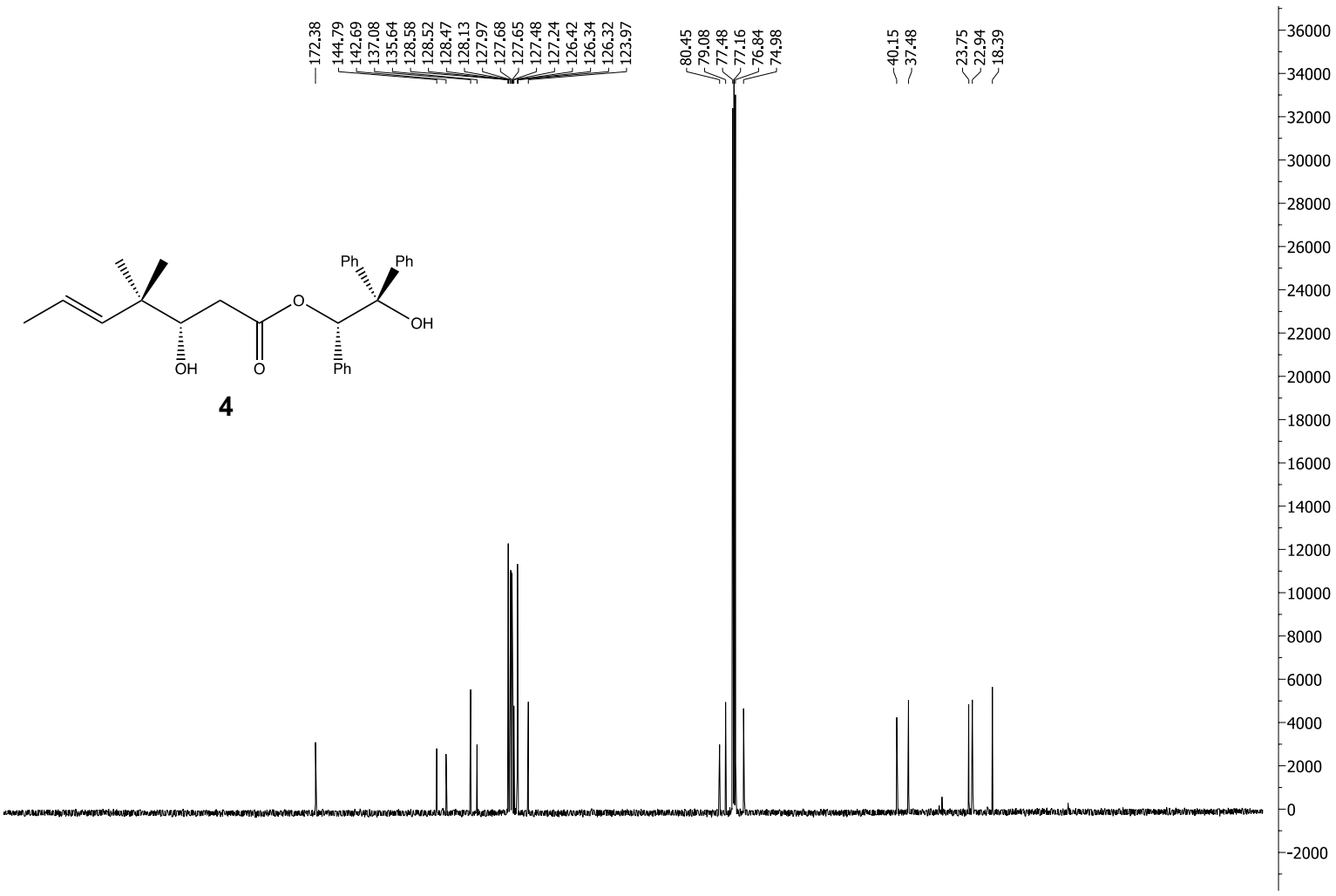

\begin{tabular}{llllllllllllllllllllllllllllllllll}
\hline 240 & 230 & 220 & 210 & 200 & 190 & 180 & 170 & 160 & 150 & 140 & 130 & 120 & 110 & 100 & 90 & 80 & 70 & 60 & 50 & 40 & 30 & 20 & 10 & 0 & -10 & -20 & -30 & -40
\end{tabular} 
${ }^{1} \mathrm{H} \mathrm{NMR}\left(400 \mathrm{MHz}, \mathrm{CDCl}_{3}\right)$

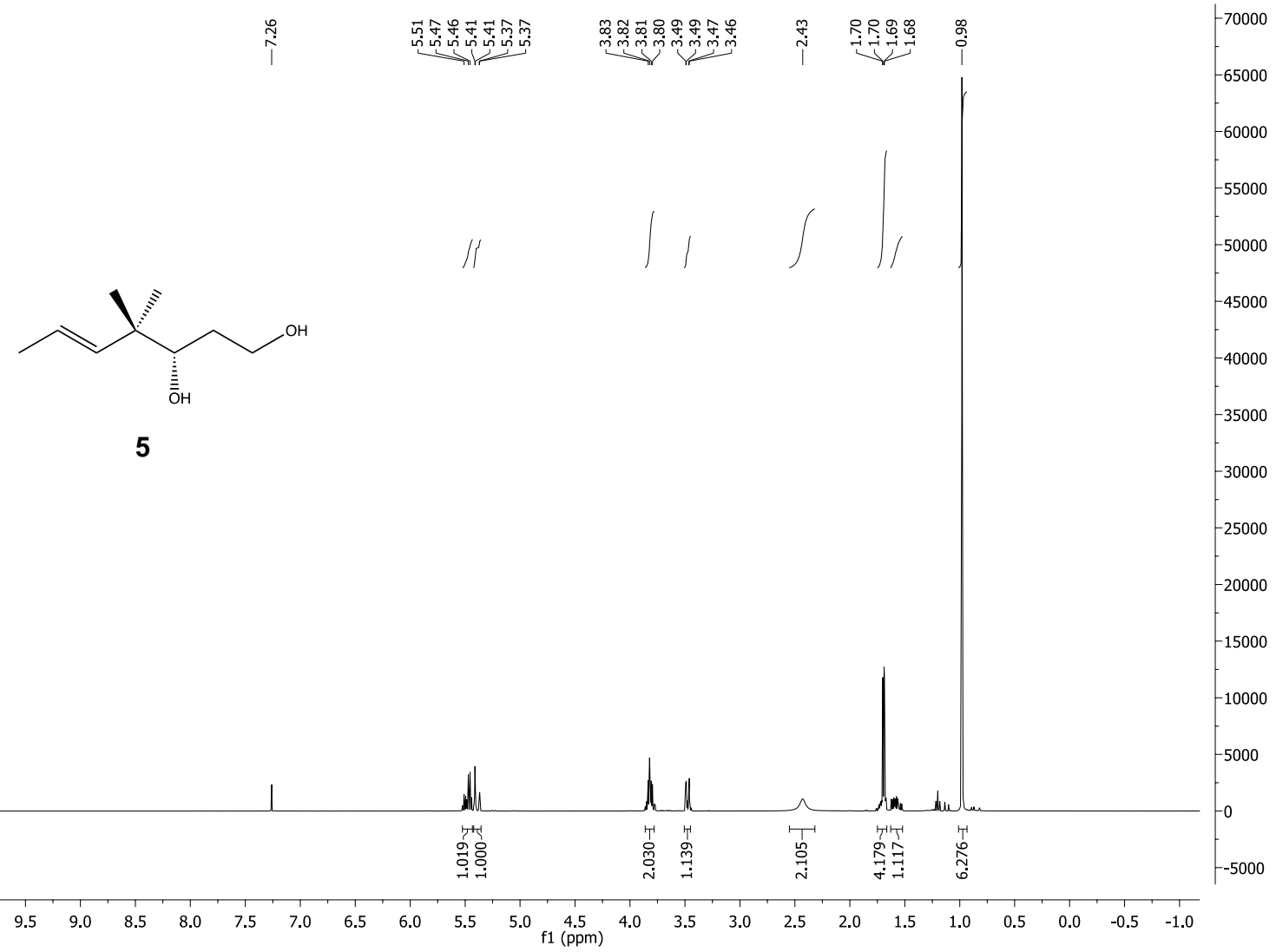

${ }^{13} \mathrm{C}$ NMR $\left(100 \mathrm{MHz}, \mathrm{CDCl}_{3}\right)$

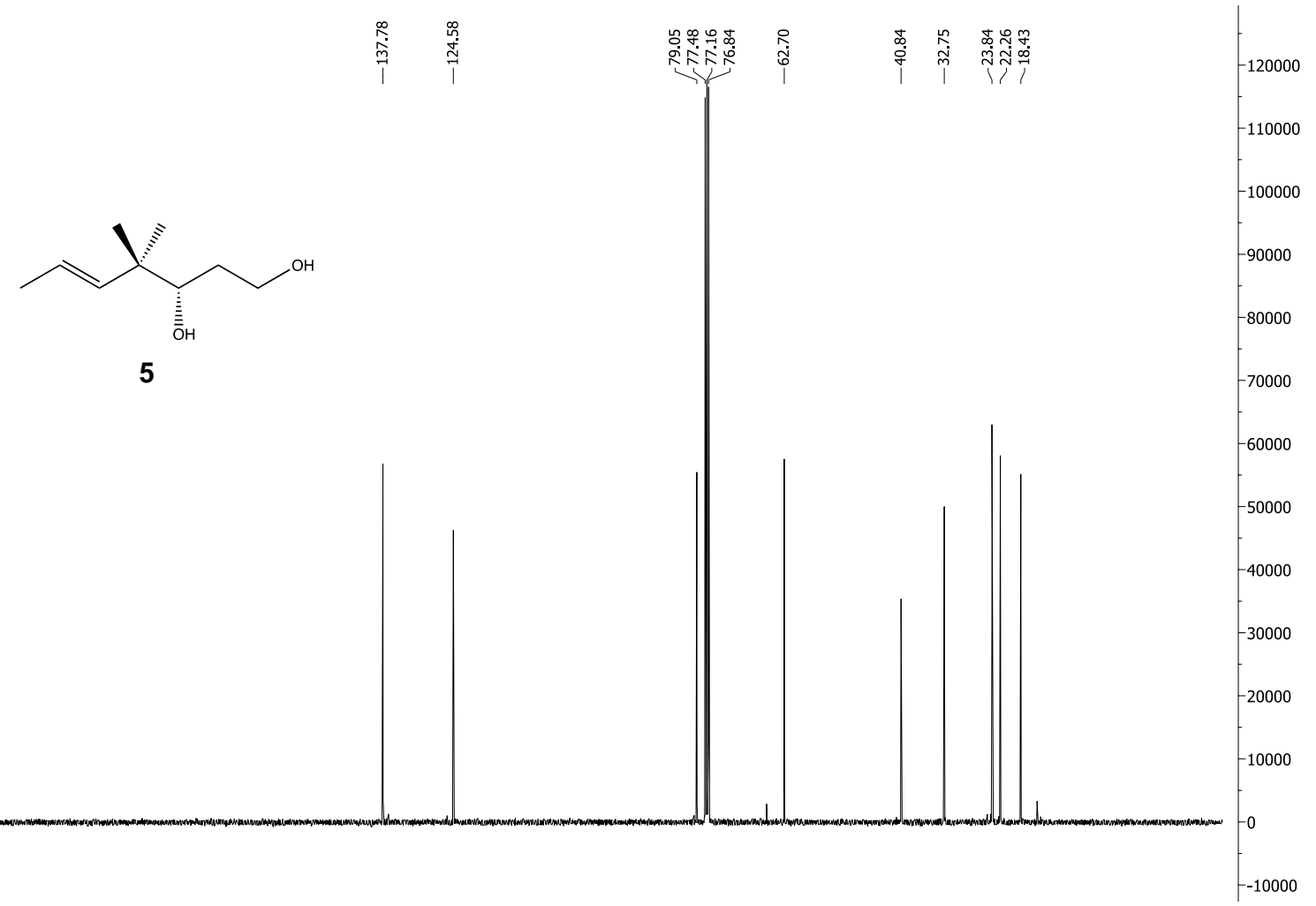

$\begin{array}{lllllllllllllllllllllllllllll}210 & 200 & 190 & 180 & 170 & 160 & 150 & 140 & 130 & 120 & 110 & 100 & 90 & 80 & 70 & 60 & 50 & 40 & 30 & 20 & 10 & 0 & -10\end{array}$ 
${ }^{1} \mathrm{H} \mathrm{NMR}\left(600 \mathrm{MHz}, \mathrm{CDCl}_{3}\right)$

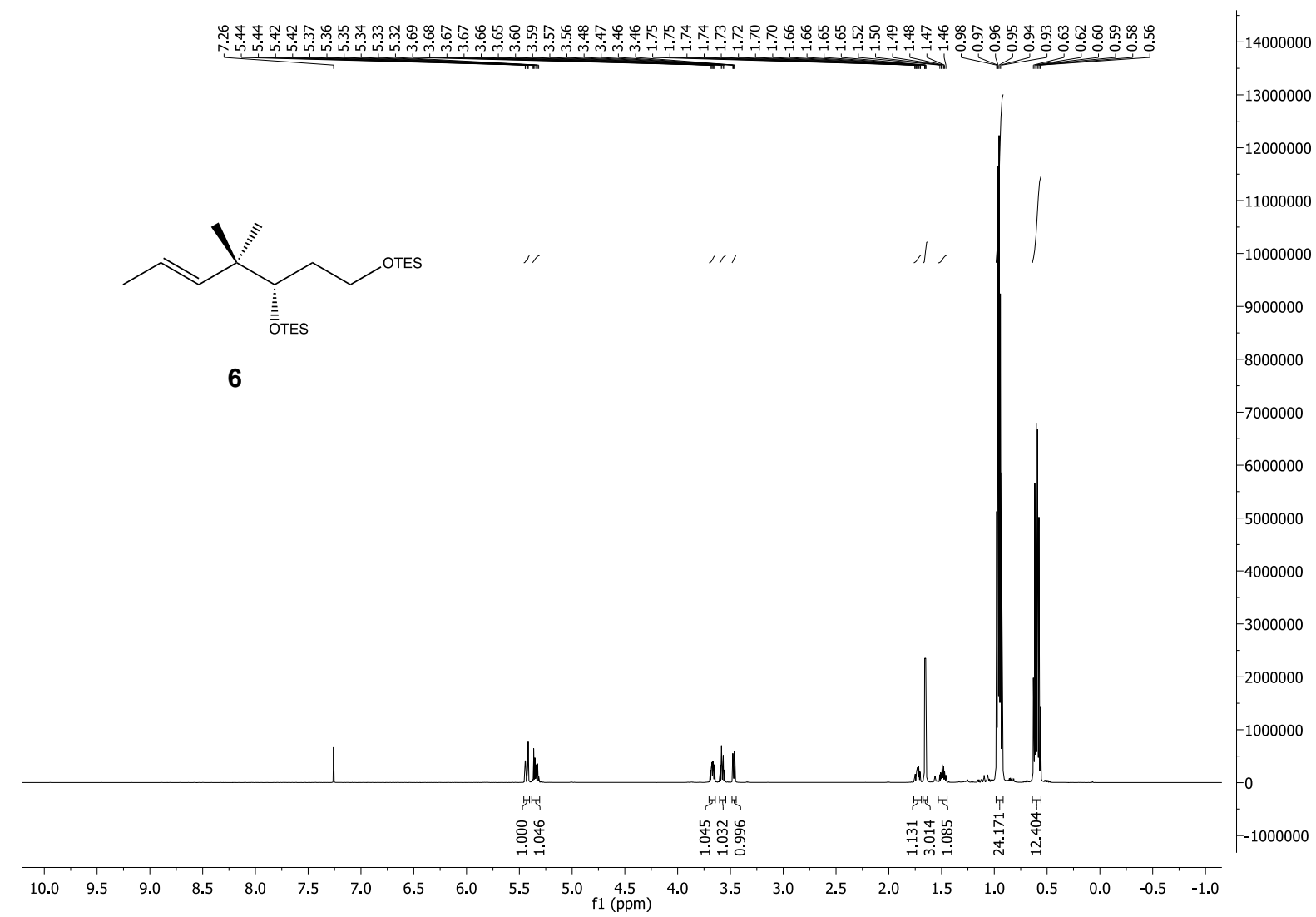

${ }^{13} \mathrm{C} \mathrm{NMR}\left(151 \mathrm{MHz}, \mathrm{CDCl}_{3}\right)$

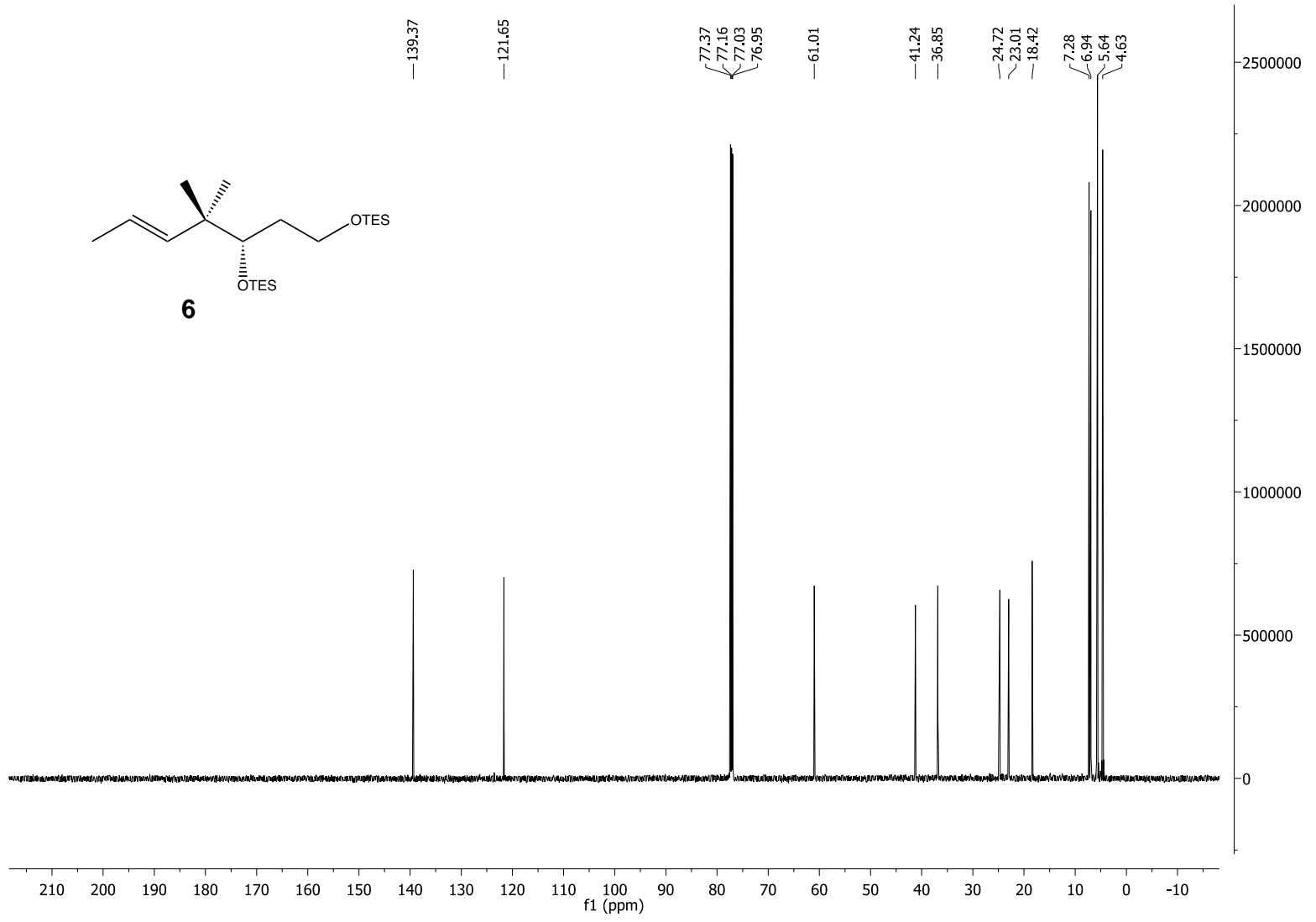


${ }^{1} \mathrm{H} \mathrm{NMR}\left(400 \mathrm{MHz}, \mathrm{CDCl}_{3}\right)$

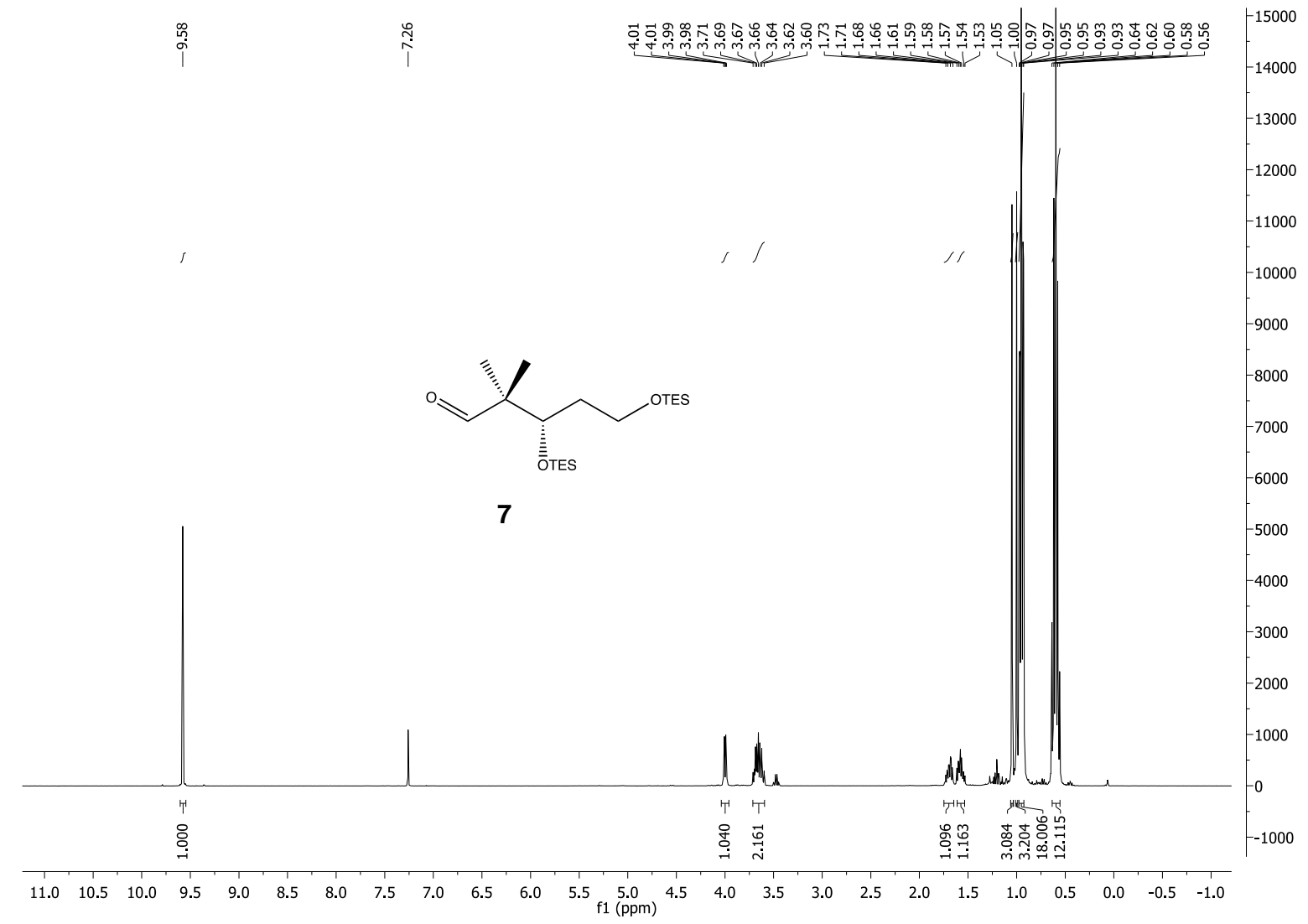

${ }^{13} \mathrm{C}$ NMR $\left(100 \mathrm{MHz}, \mathrm{CDCl}_{3}\right)$

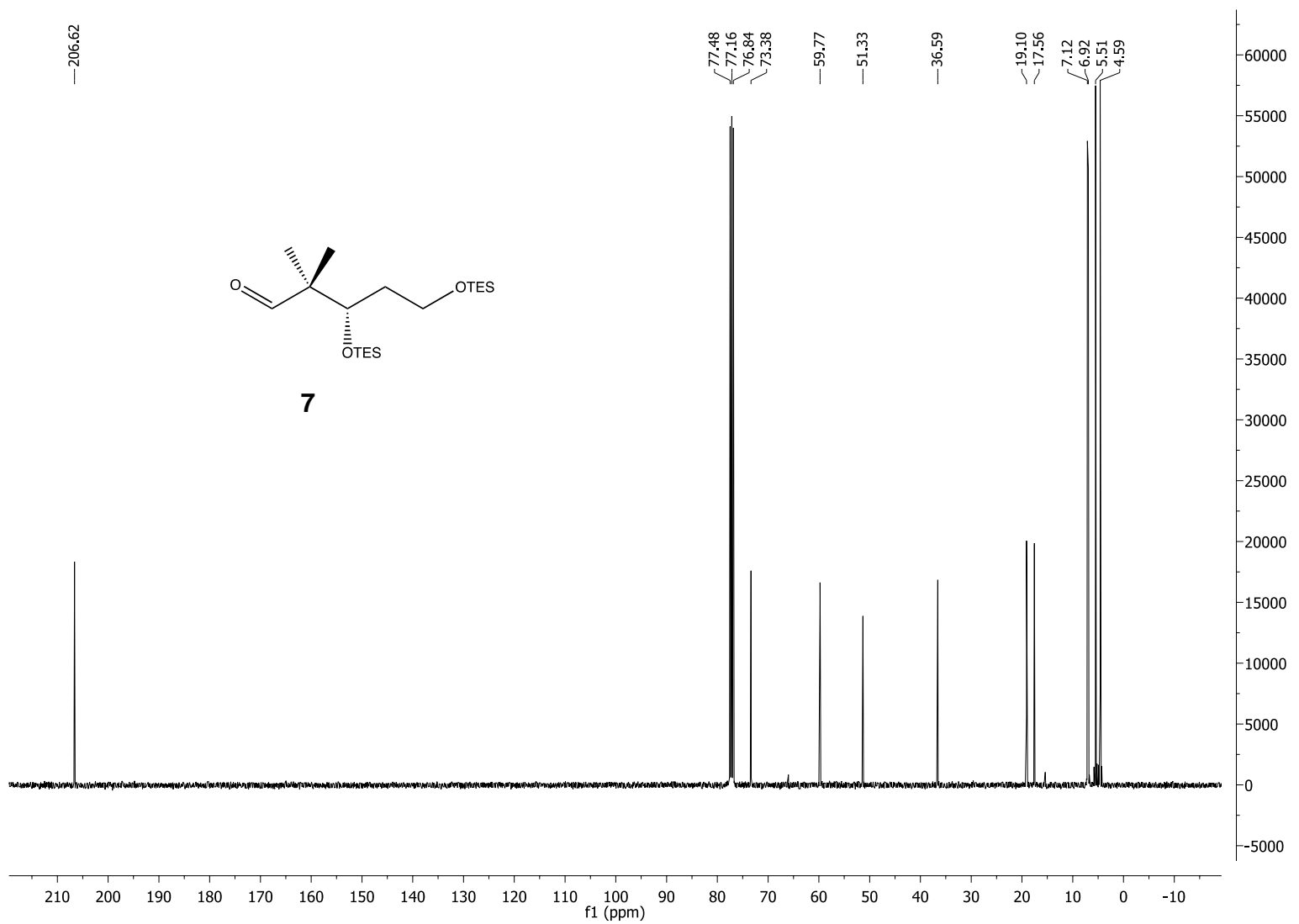


${ }^{1} \mathrm{H} \mathrm{NMR}\left(600 \mathrm{MHz}, \mathrm{CDCl}_{3}\right)$

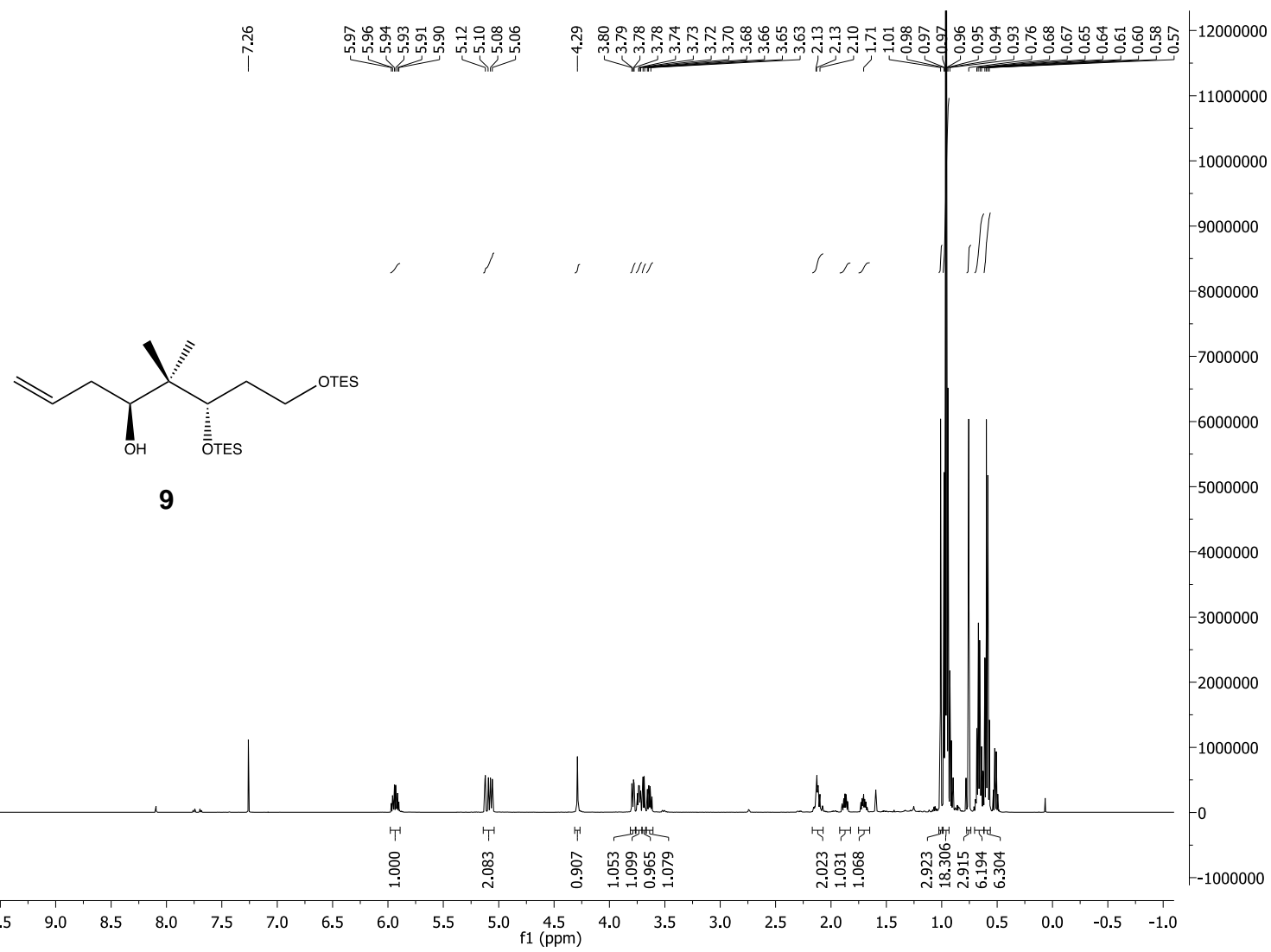

${ }^{13} \mathrm{C} \mathrm{NMR}\left(151 \mathrm{MHz}, \mathrm{CDCl}_{3}\right)$

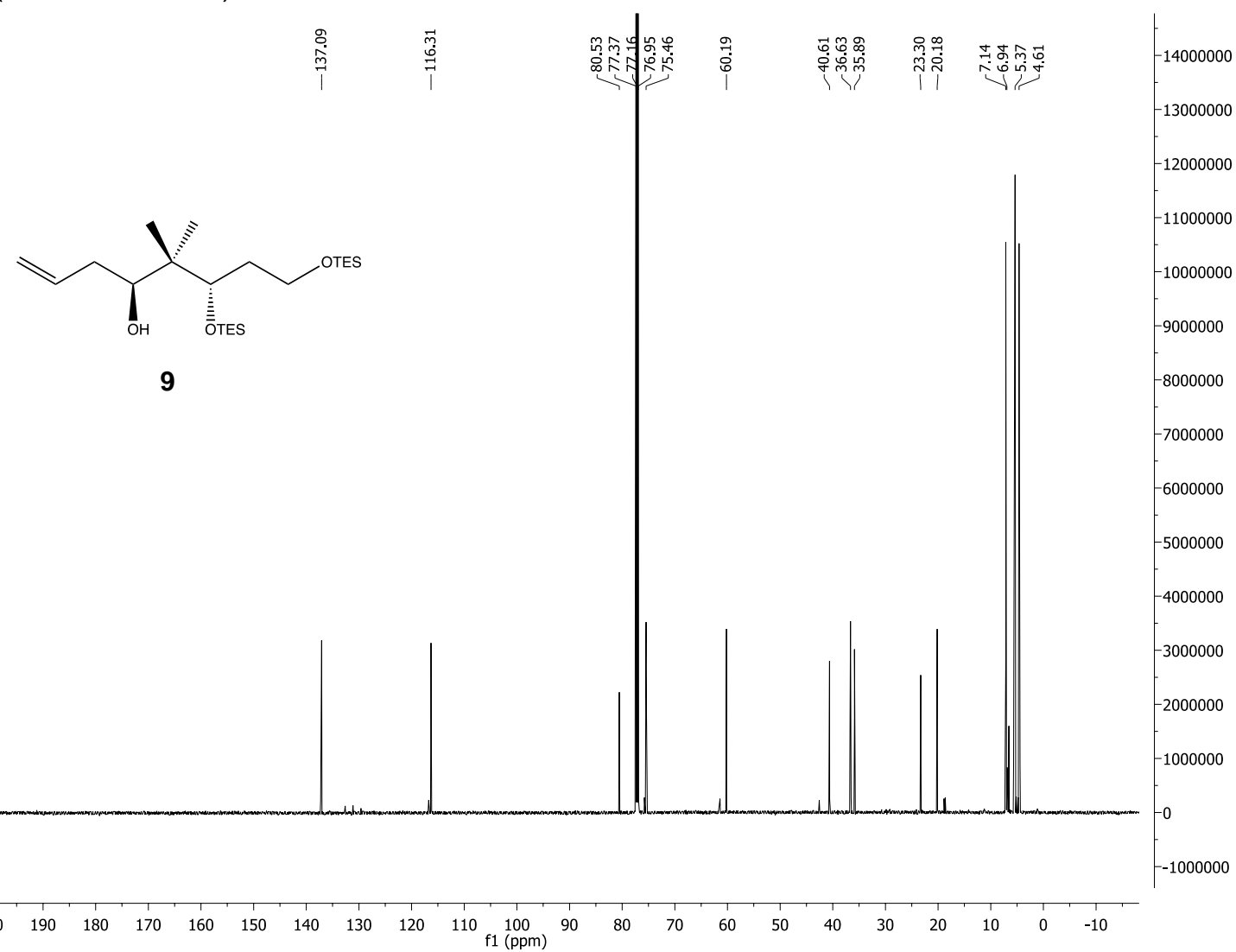


${ }^{1} \mathrm{H} \mathrm{NMR}\left(600 \mathrm{MHz}, \mathrm{CDCl}_{3}\right)$

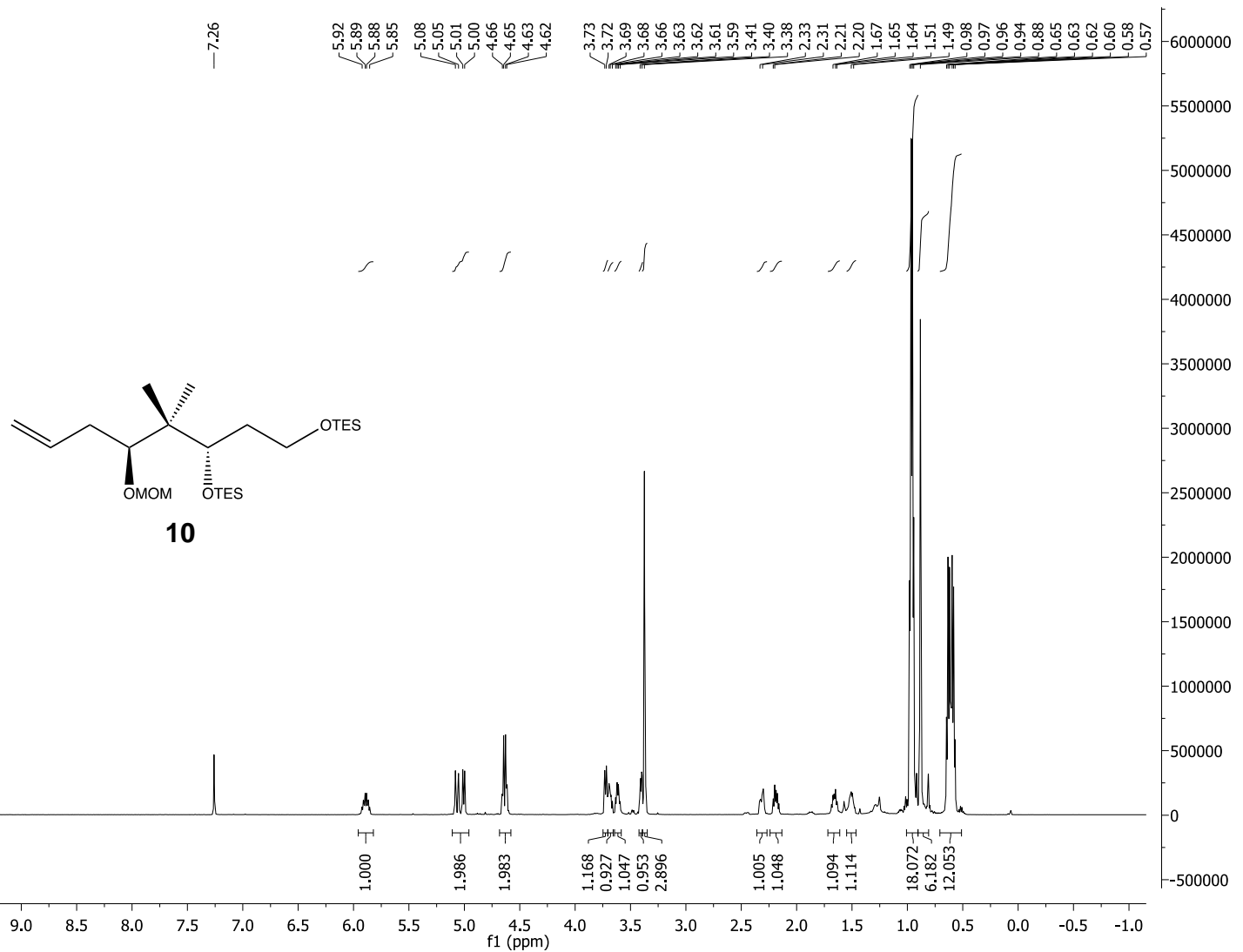

${ }^{13} \mathrm{C} \mathrm{NMR}\left(151 \mathrm{MHz}, \mathrm{CDCl}_{3}\right)$

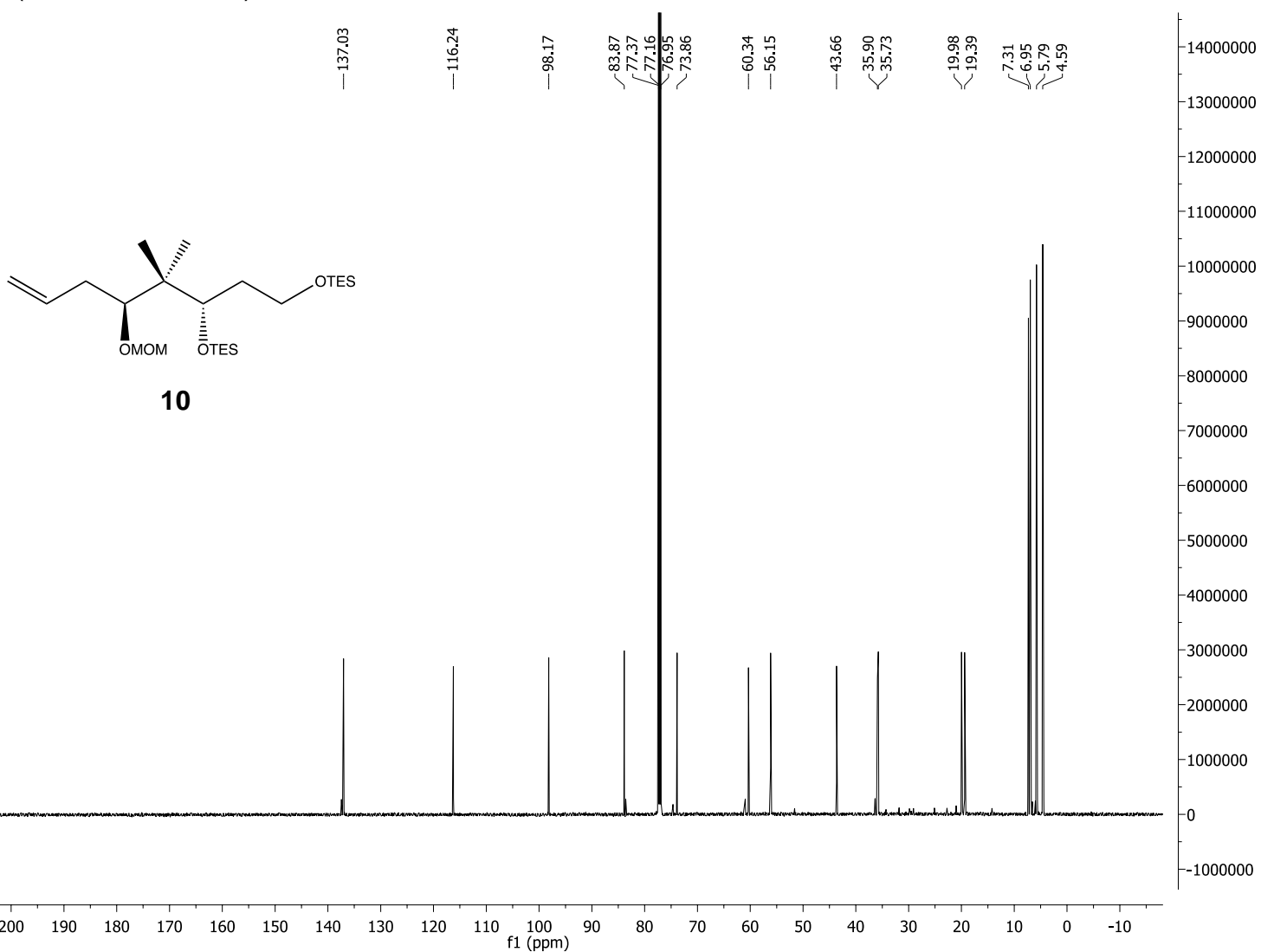


${ }^{1} \mathrm{H} \mathrm{NMR}\left(400 \mathrm{MHz}, \mathrm{CDCl}_{3}\right)$

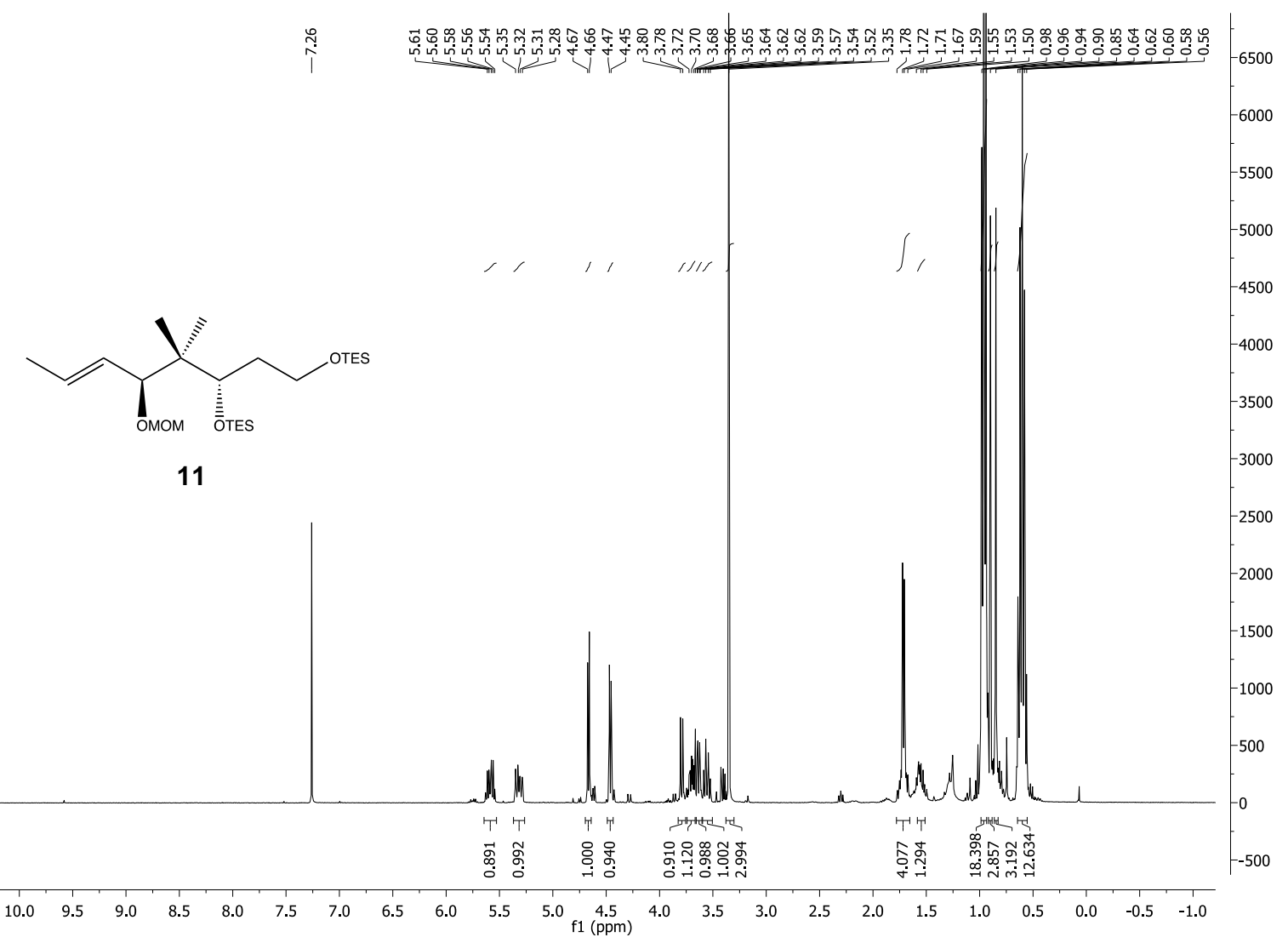

${ }^{13} \mathrm{C}$ NMR $\left(100 \mathrm{MHz}, \mathrm{CDCl}_{3}\right)$

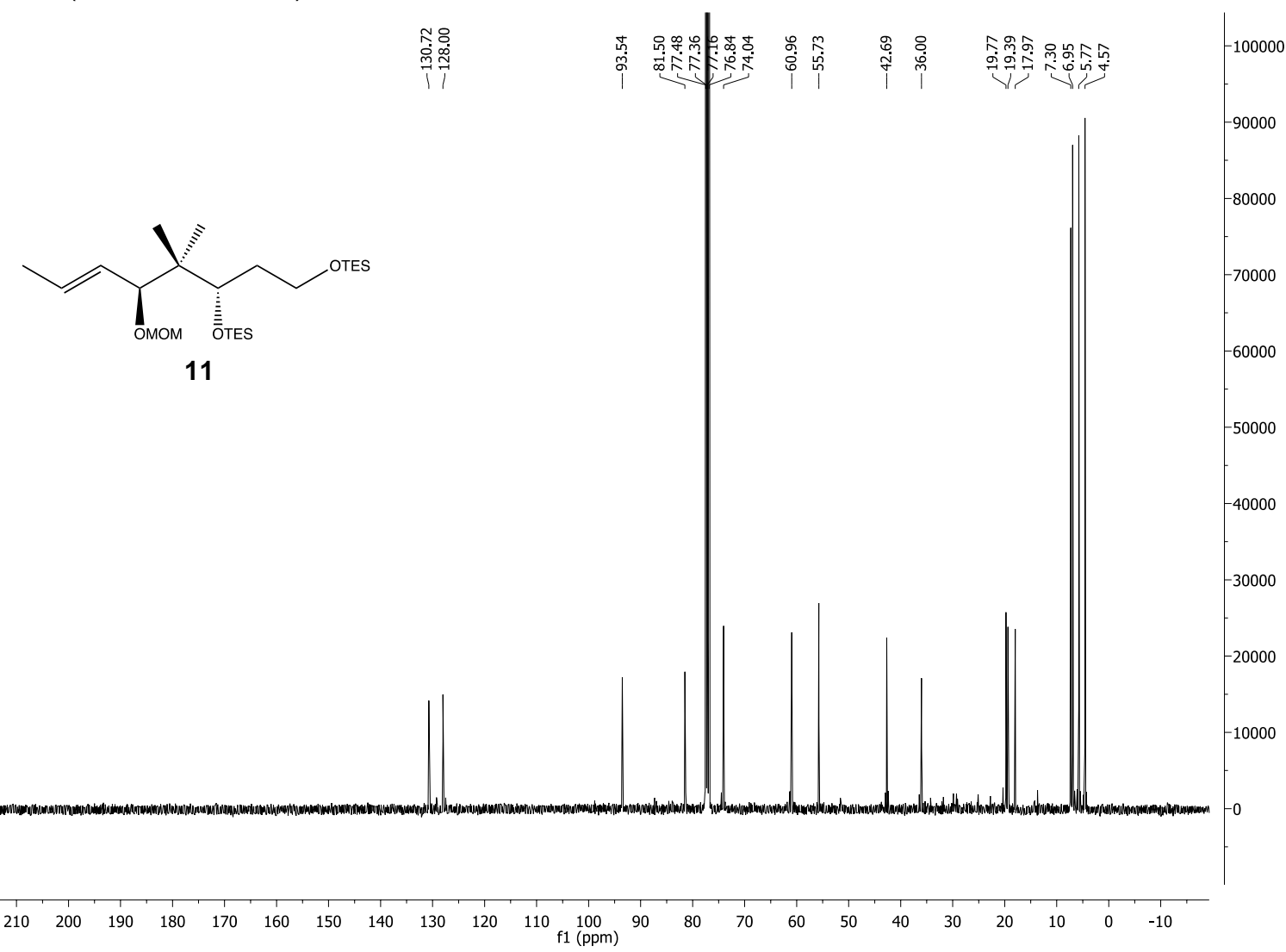




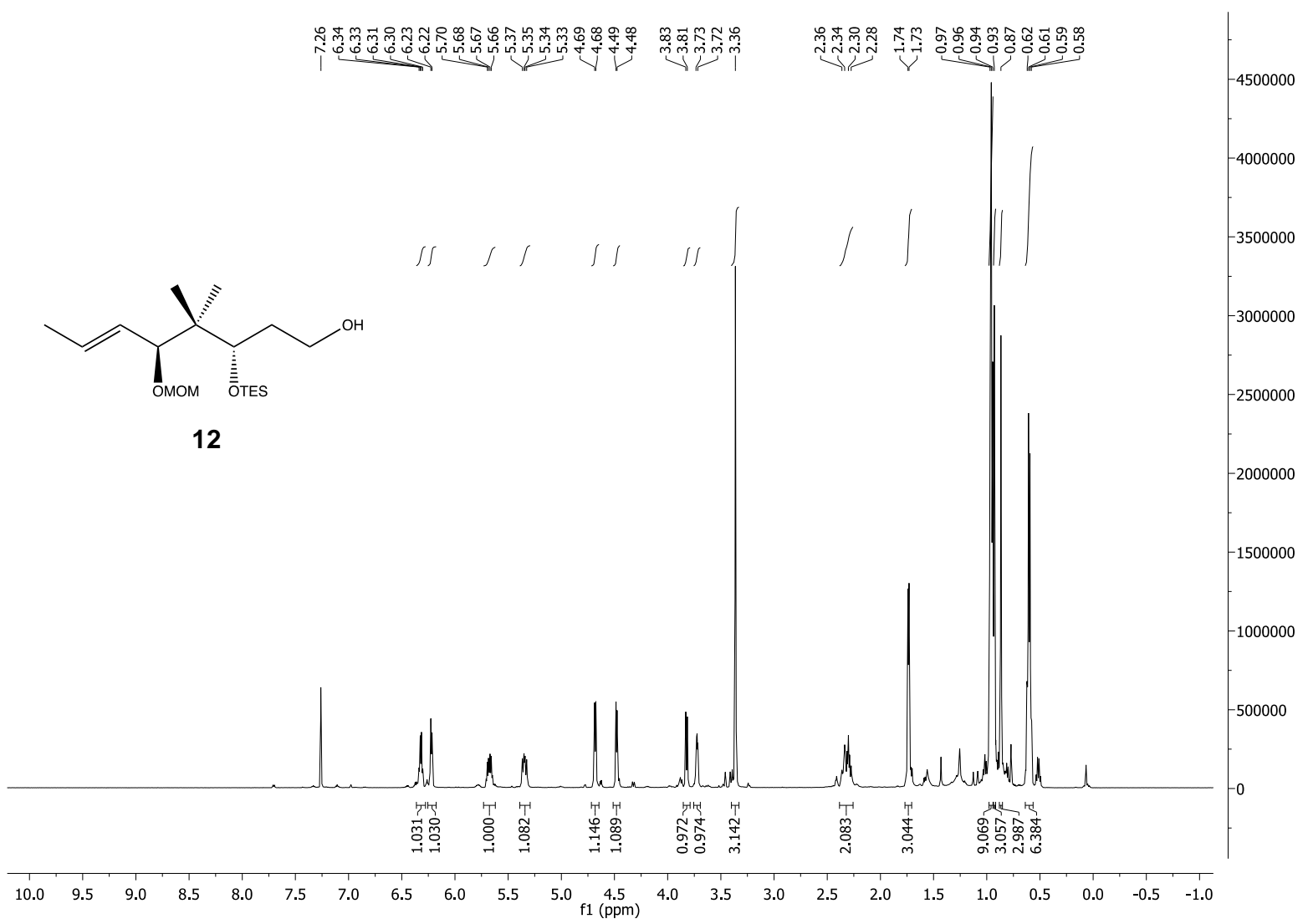

${ }^{13} \mathrm{C}$ NMR $\left(100 \mathrm{MHz}, \mathrm{CDCl}_{3}\right)$

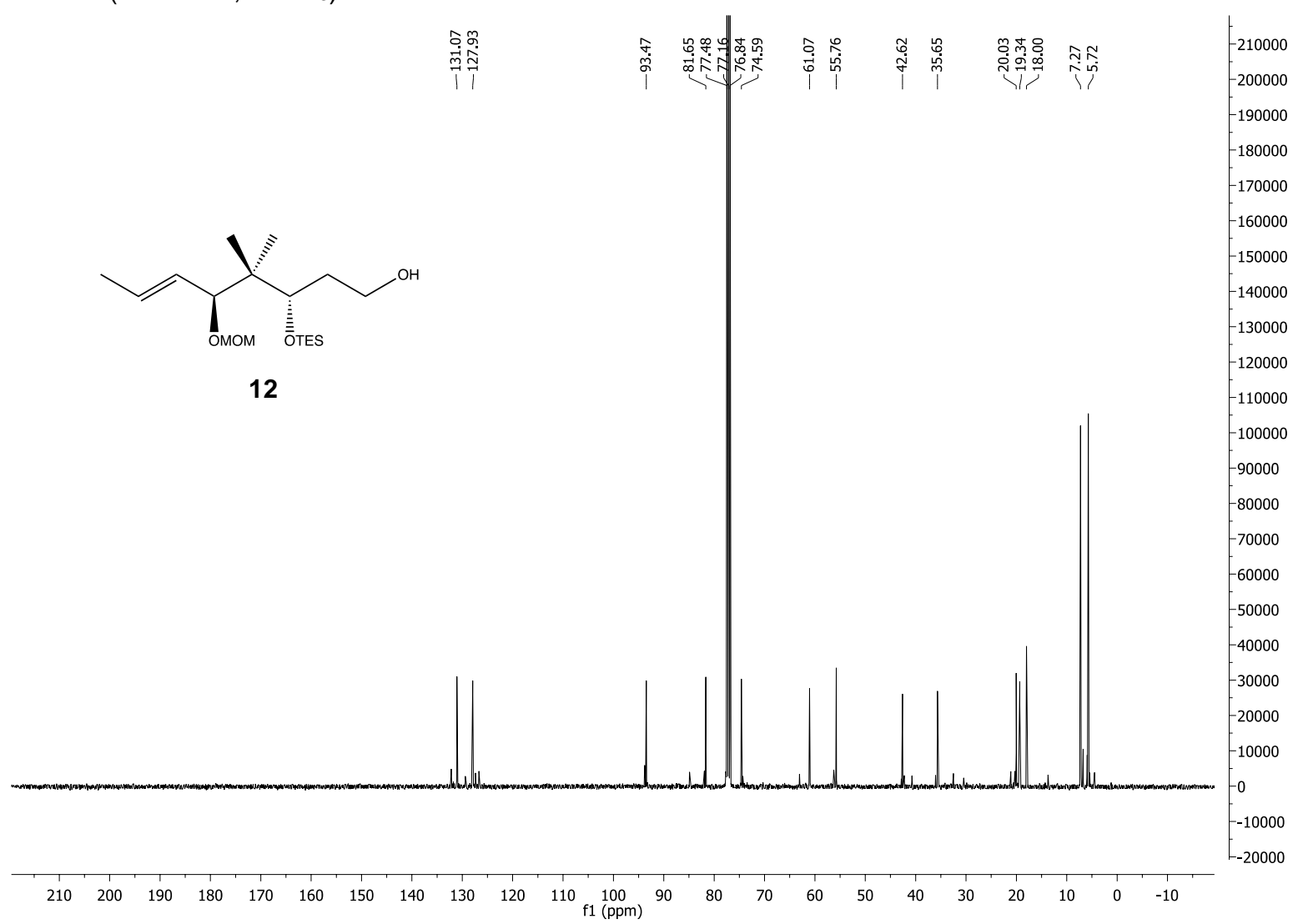


${ }^{1} \mathrm{H}$ NMR $\left(600 \mathrm{MHz}, \mathrm{CDCl}_{3}\right)$

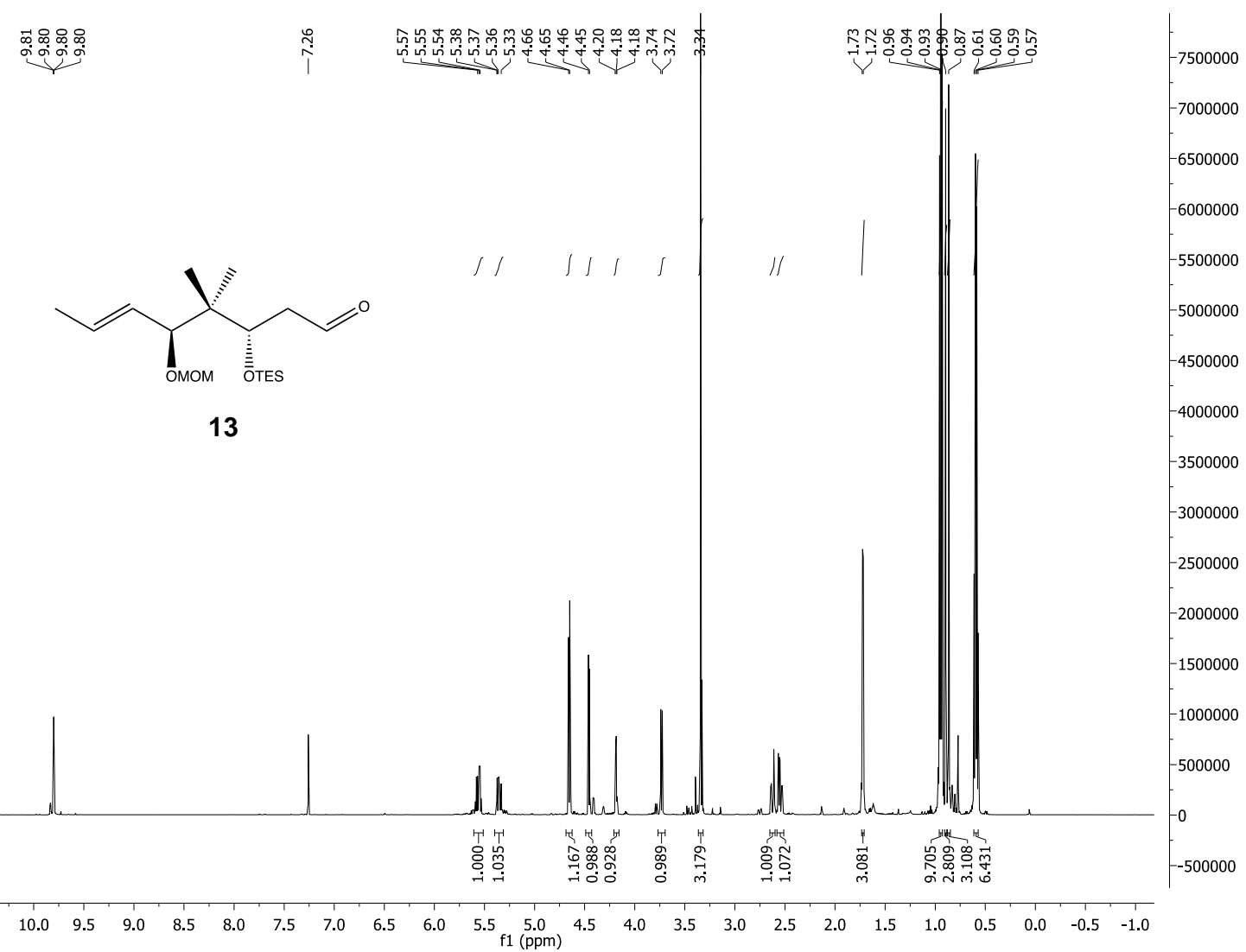

${ }^{13} \mathrm{C}$ NMR $\left(151 \mathrm{MHz}, \mathrm{CDCl}_{3}\right)$

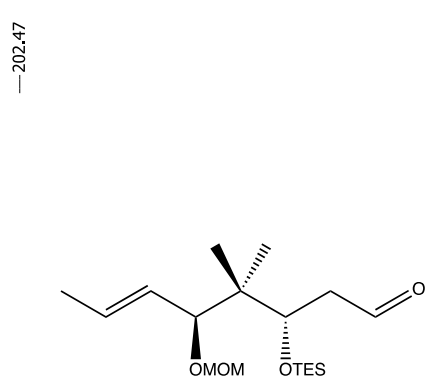

13

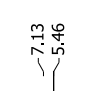

$-8000000$ $-7500000$ $-7000000$ $-6500000$ $-6000000$ $-5500000$ $-5000000$ $-4500000$ $-4000000$ $-3500000$ $-3000000$ $-2500000$

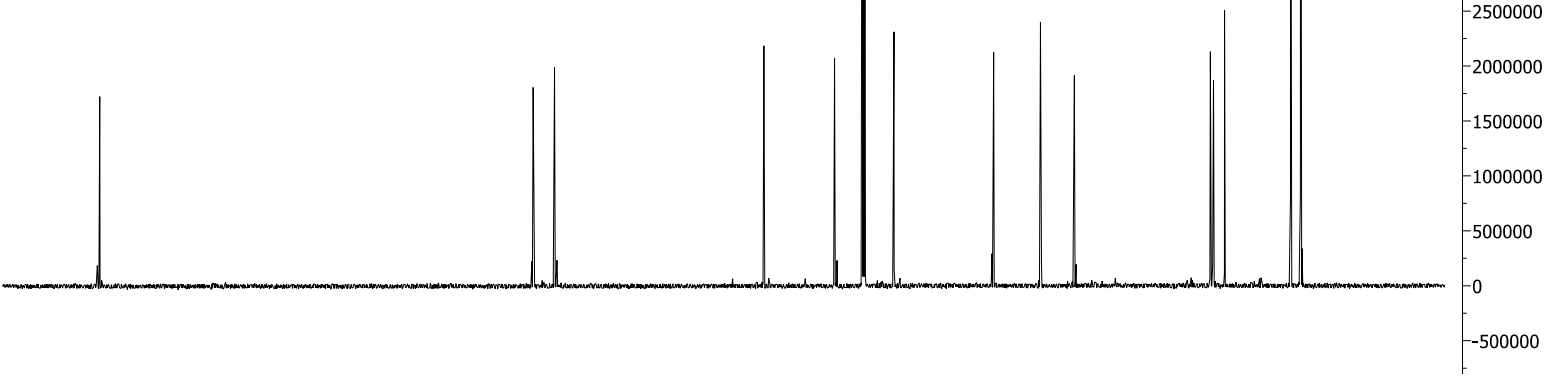

$\begin{array}{llllllllllllllllllllllllll} & 210 & 200 & 190 & 180 & 170 & 160 & 150 & 140 & 130 & 120 & 110 & 100 & 90 & 80 & 70 & 60 & 50 & 40 & 30 & 20 & 10 & 0 & -10\end{array}$ 


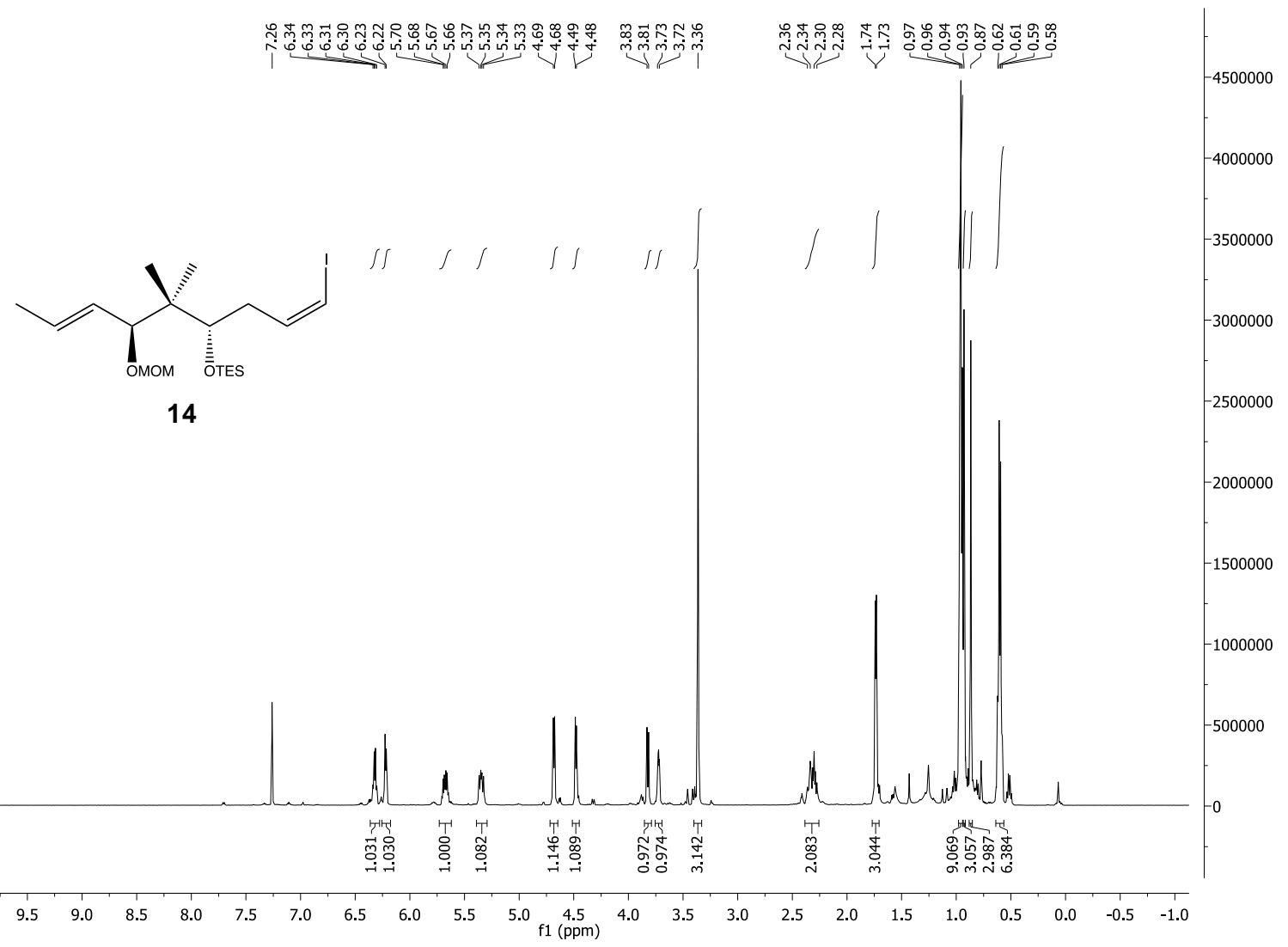

${ }^{13} \mathrm{C}$ NMR (151 MHz, CDCl 3 )

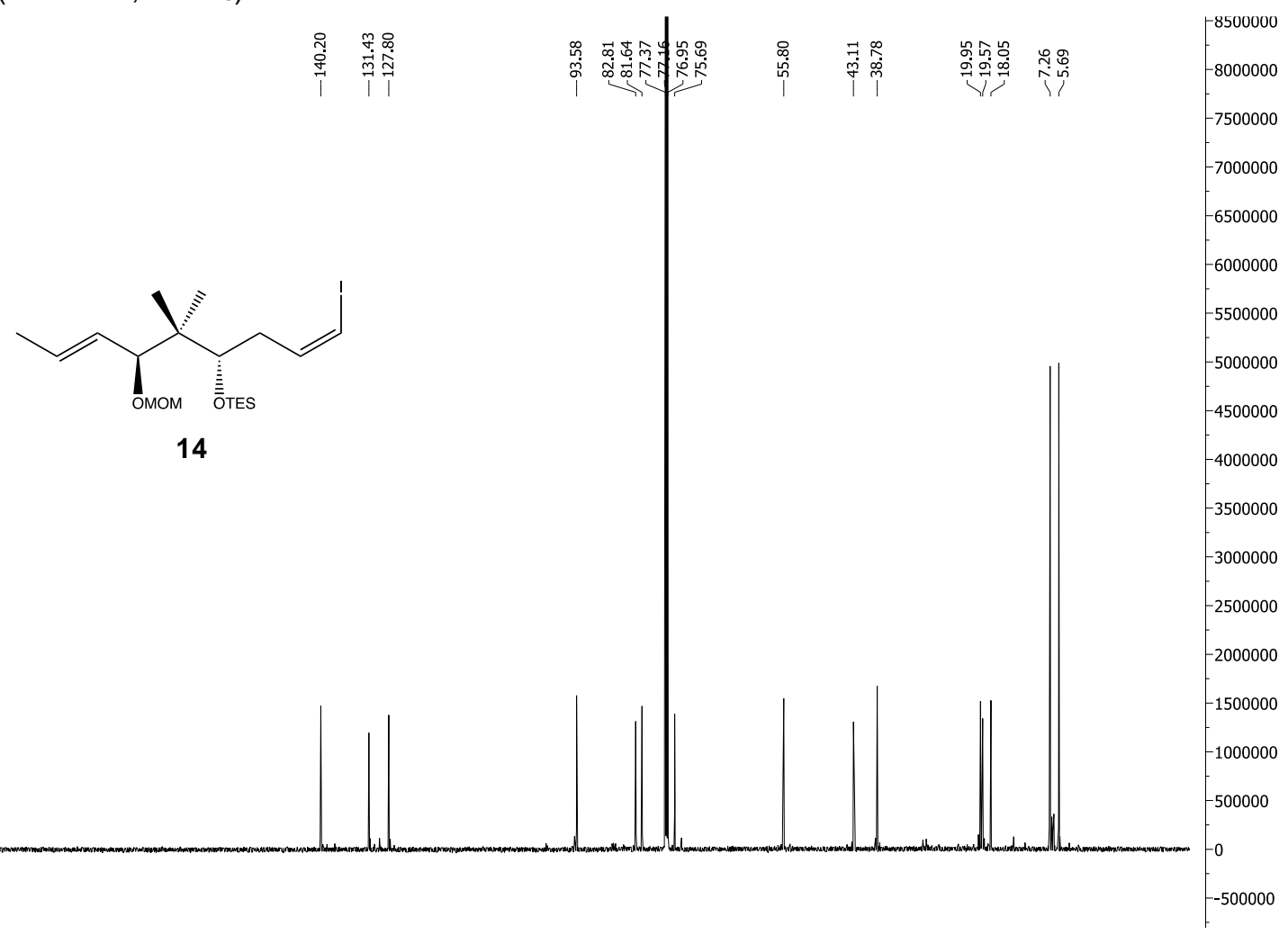

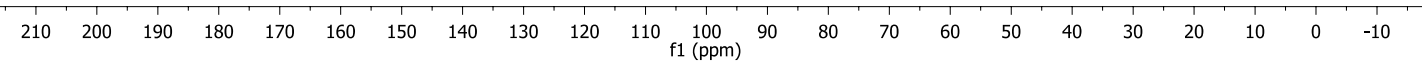


${ }^{1} \mathrm{H} \mathrm{NMR}\left(400 \mathrm{MHz}, \mathrm{CDCl}_{3}\right)$

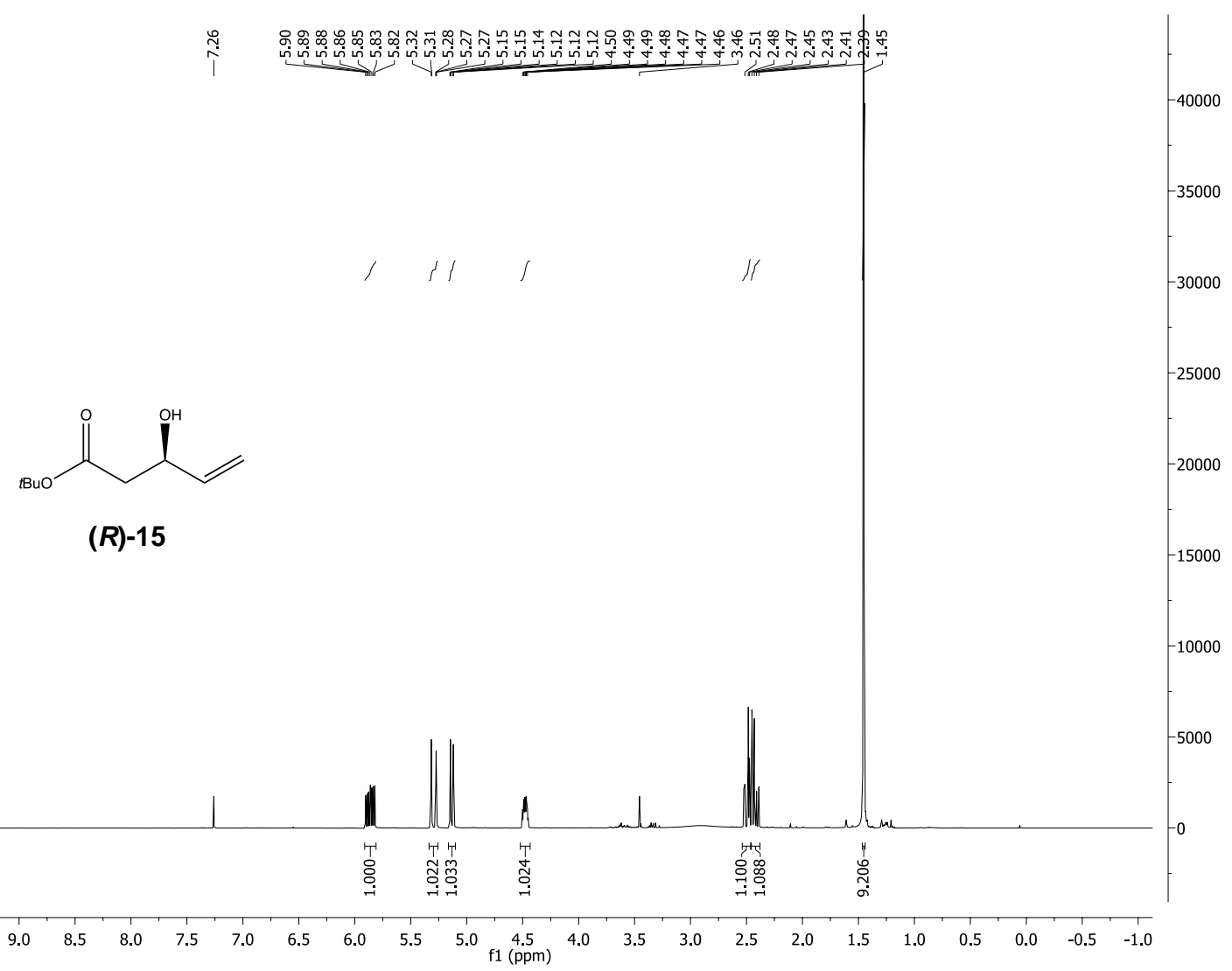

${ }^{13} \mathrm{C}$ NMR $\left(100 \mathrm{MHz}, \mathrm{CDCl}_{3}\right)$

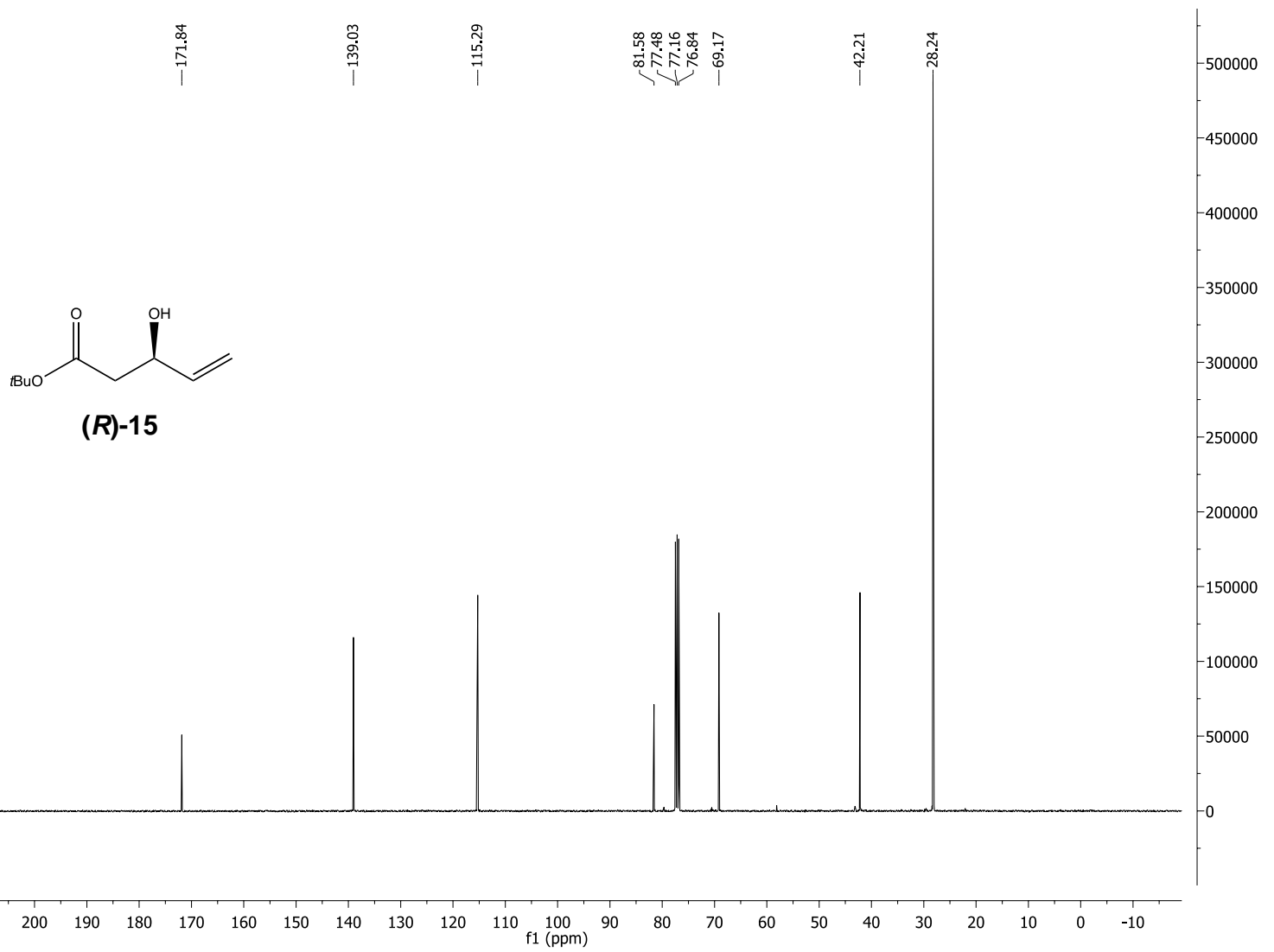


${ }^{1} \mathrm{H}$ NMR $\left(400 \mathrm{MHz}, \mathrm{CDCl}_{3}\right)$

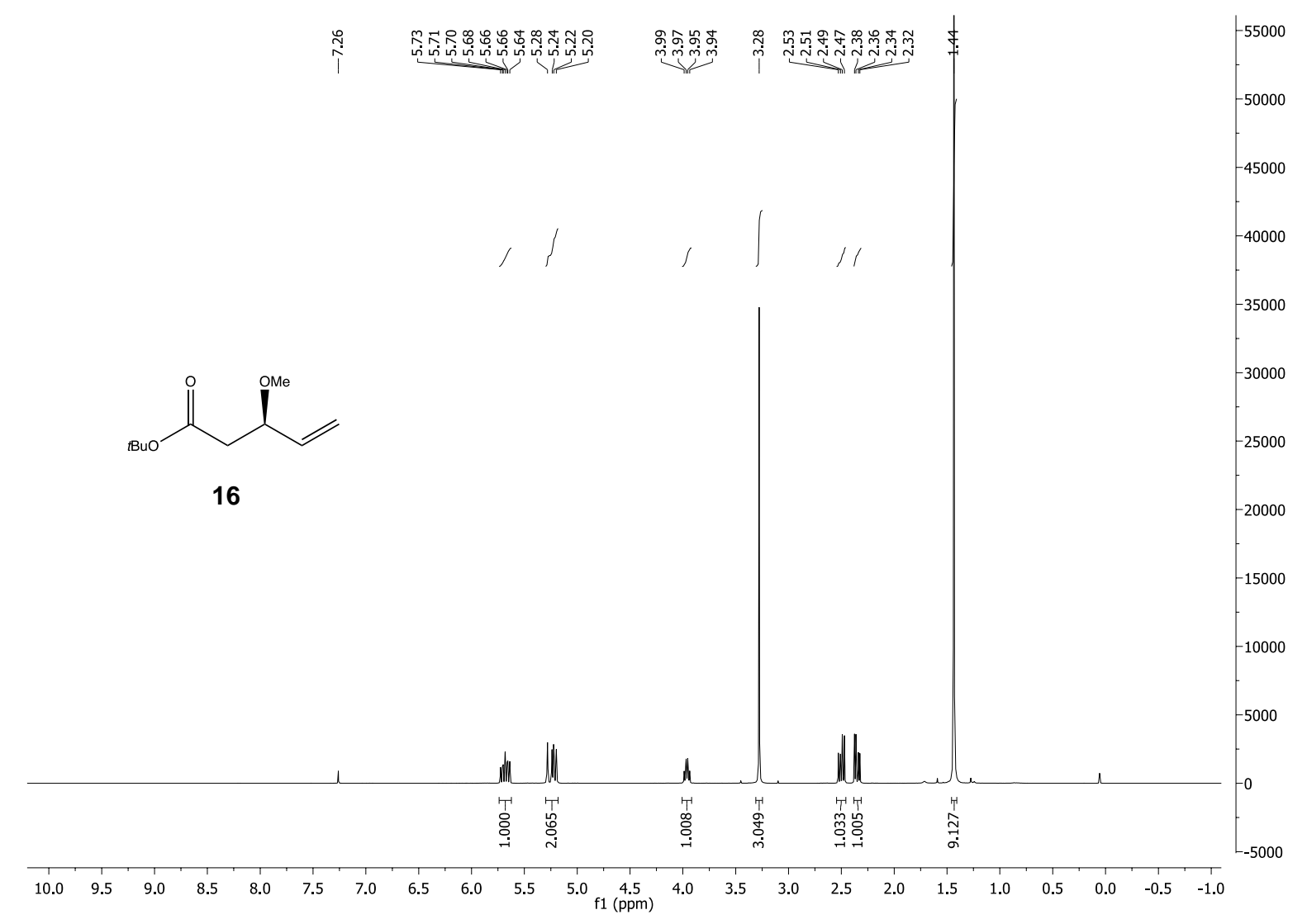

${ }^{13} \mathrm{C} \mathrm{NMR}\left(100 \mathrm{MHz}, \mathrm{CDCl}_{3}\right)$

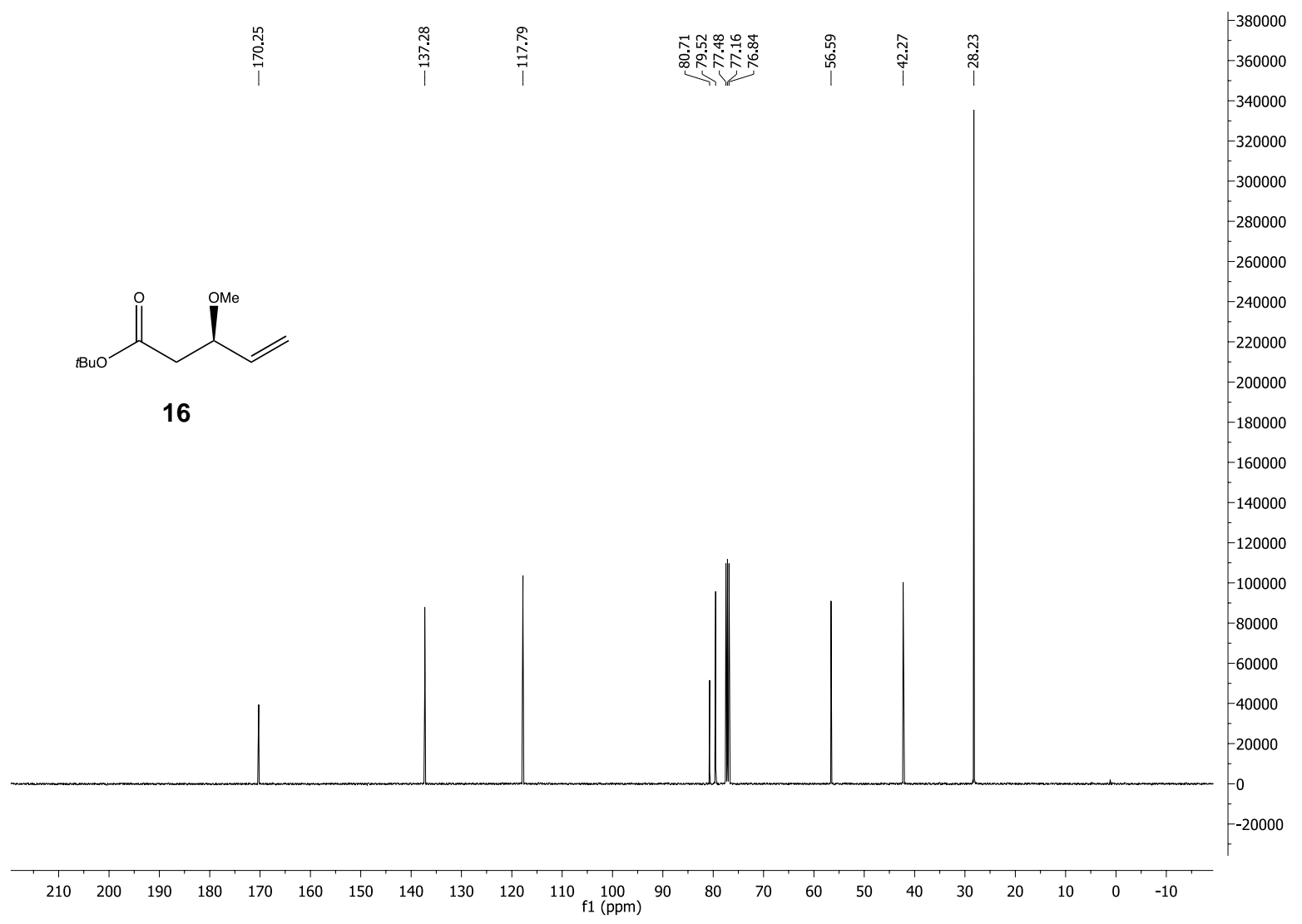


${ }^{1} \mathrm{H} \mathrm{NMR}\left(600 \mathrm{MHz}, \mathrm{CDCl}_{3}\right)$

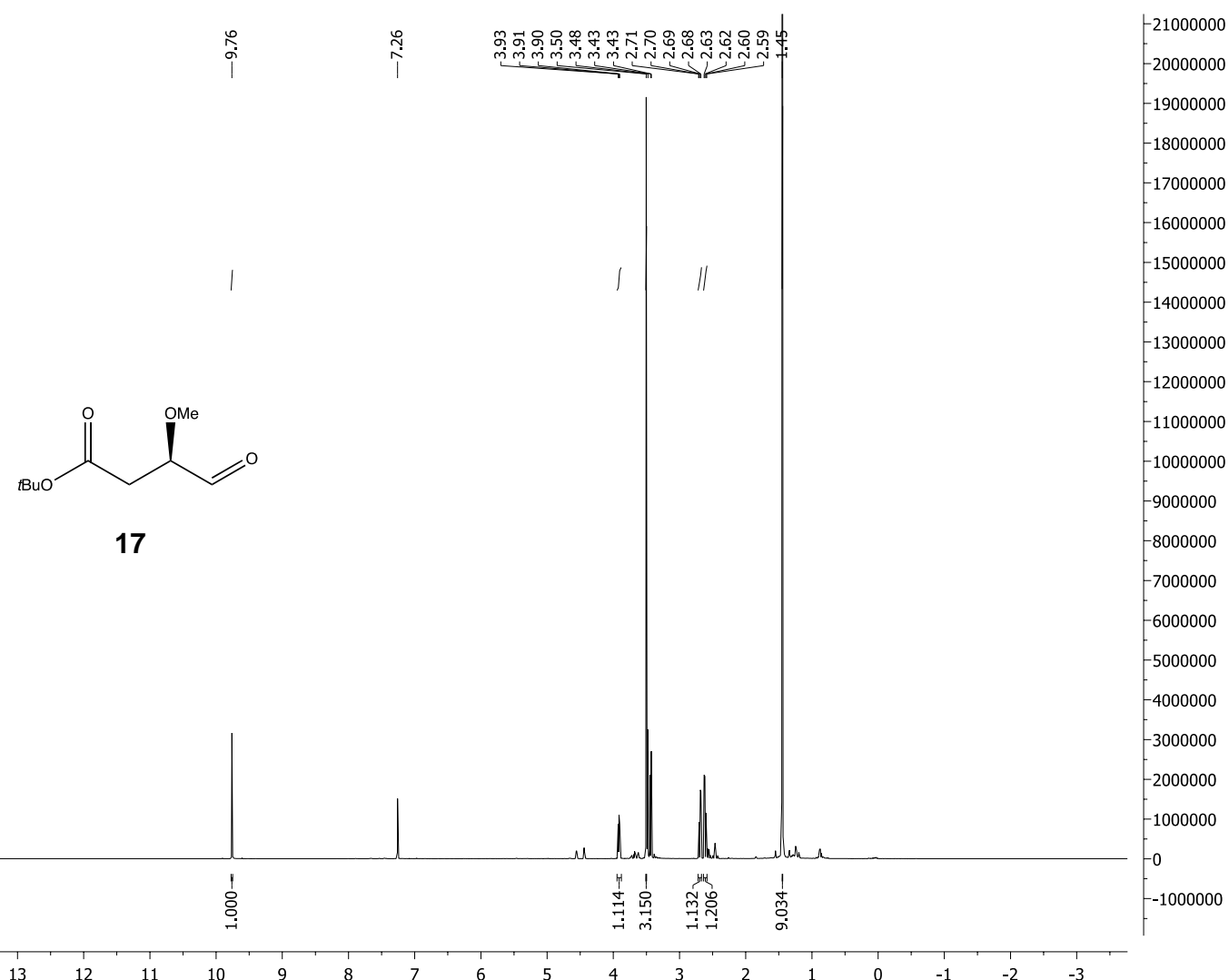

${ }^{13} \mathrm{C}$ NMR $\left(151 \mathrm{MHz}, \mathrm{CDCl}_{3}\right)$

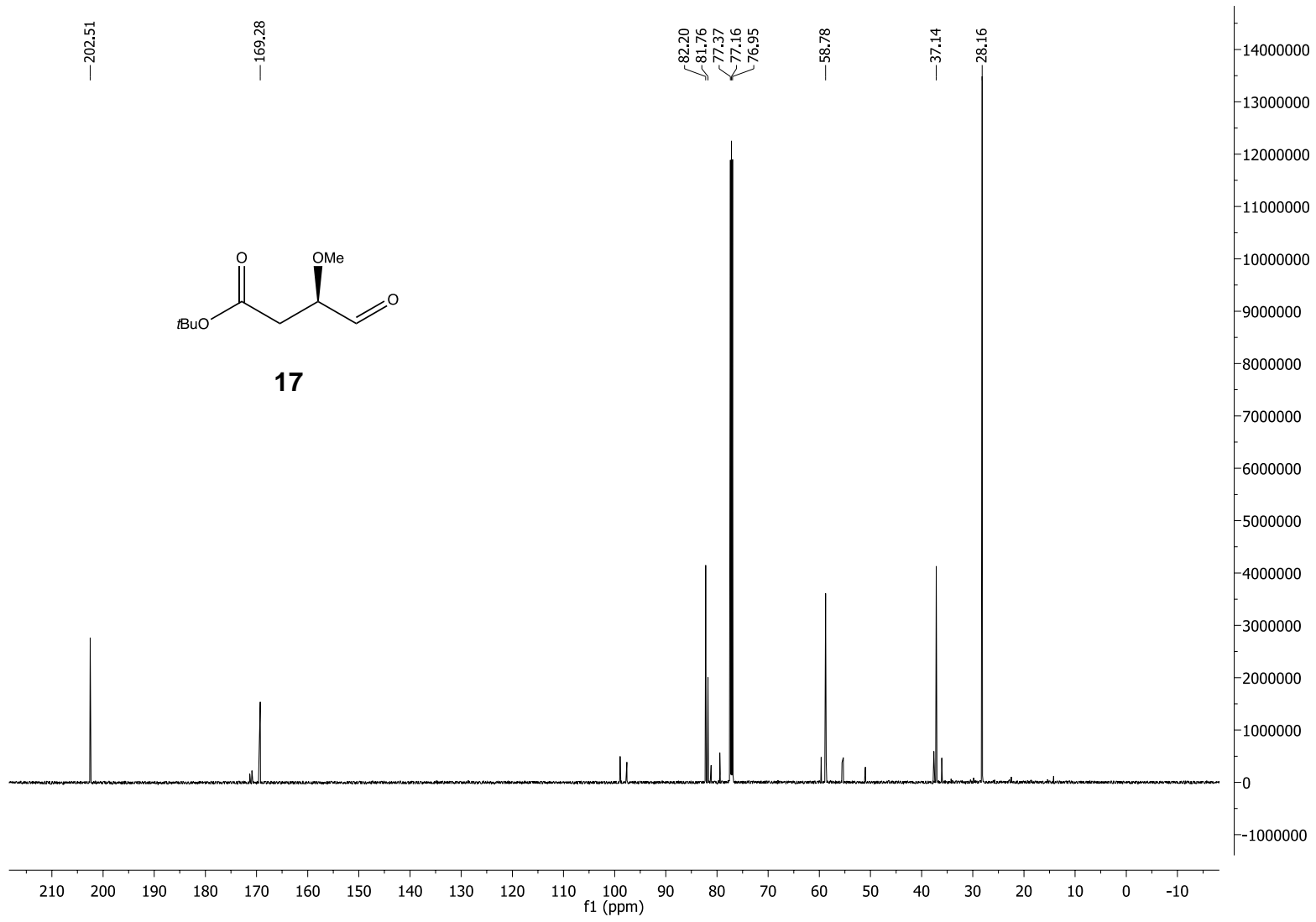


${ }^{1} \mathrm{H} \mathrm{NMR}\left(400 \mathrm{MHz}, \mathrm{CDCl}_{3}\right)$

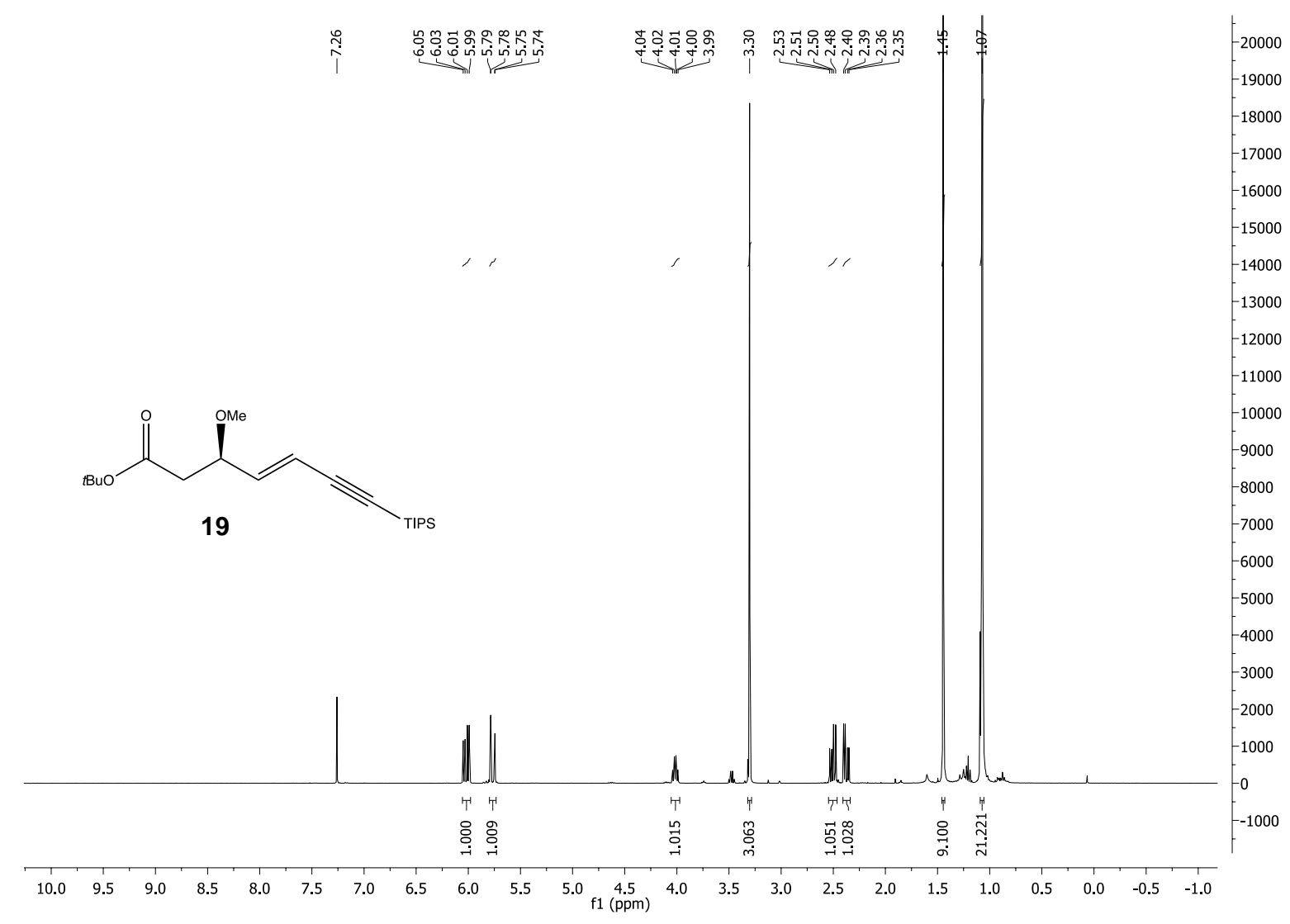

${ }^{13} \mathrm{C}$ NMR $\left(100 \mathrm{MHz}, \mathrm{CDCl}_{3}\right)$

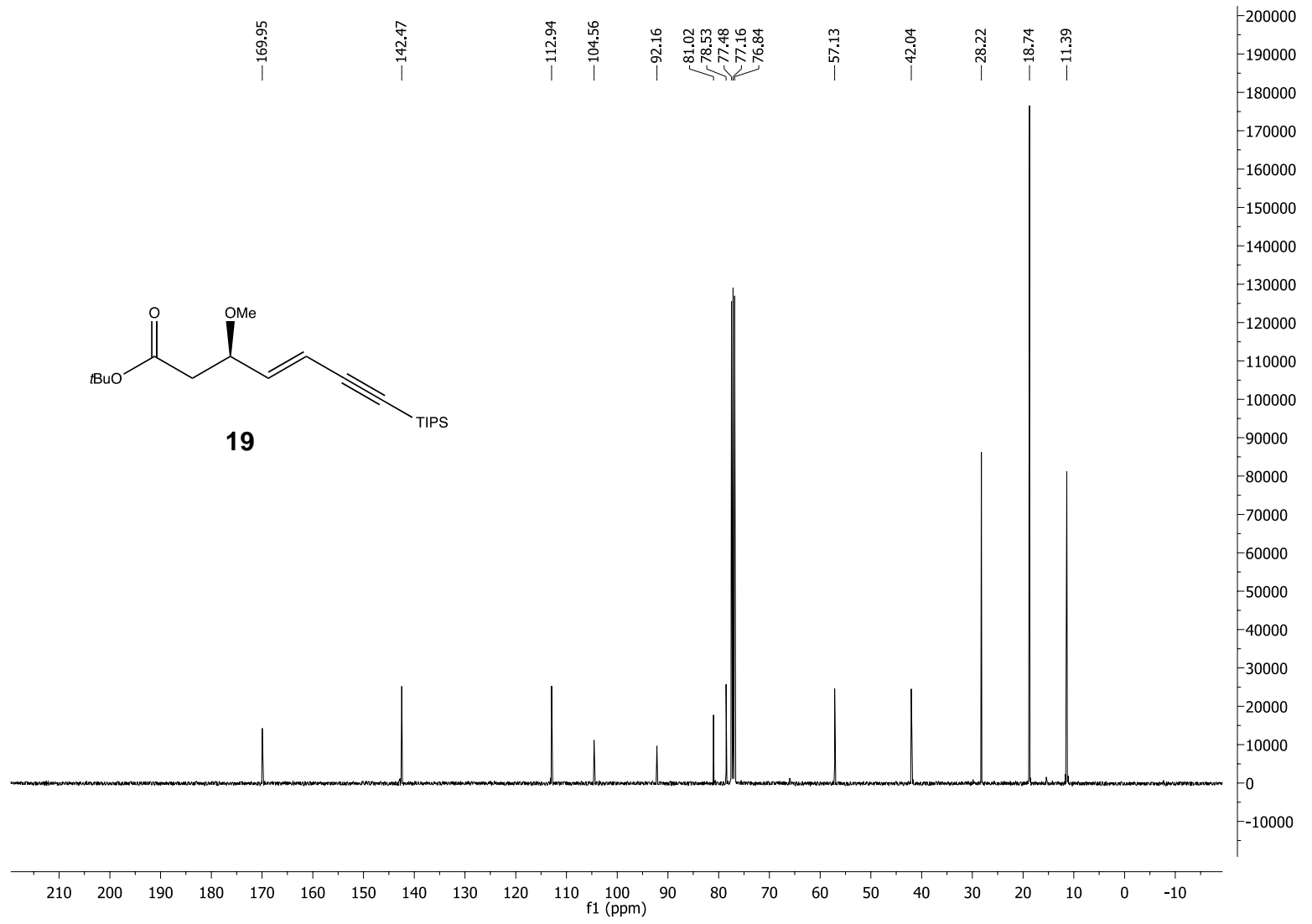


${ }^{1} \mathrm{H}$ NMR $\left(600 \mathrm{MHz}, \mathrm{CDCl}_{3}\right)$

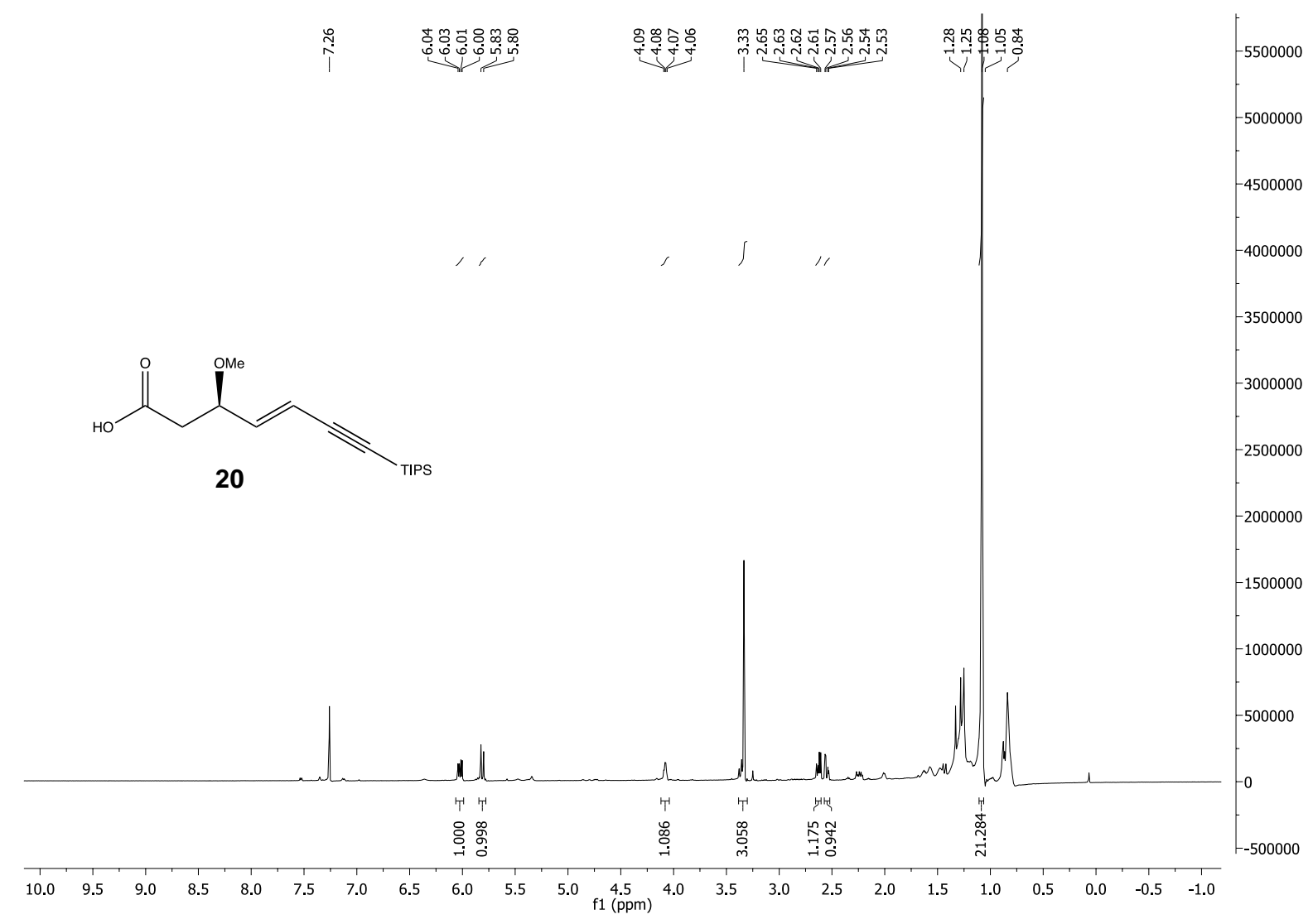

${ }^{13} \mathrm{C}$ NMR (151 MHz, $\left.\mathrm{CDCl}_{3}\right)$

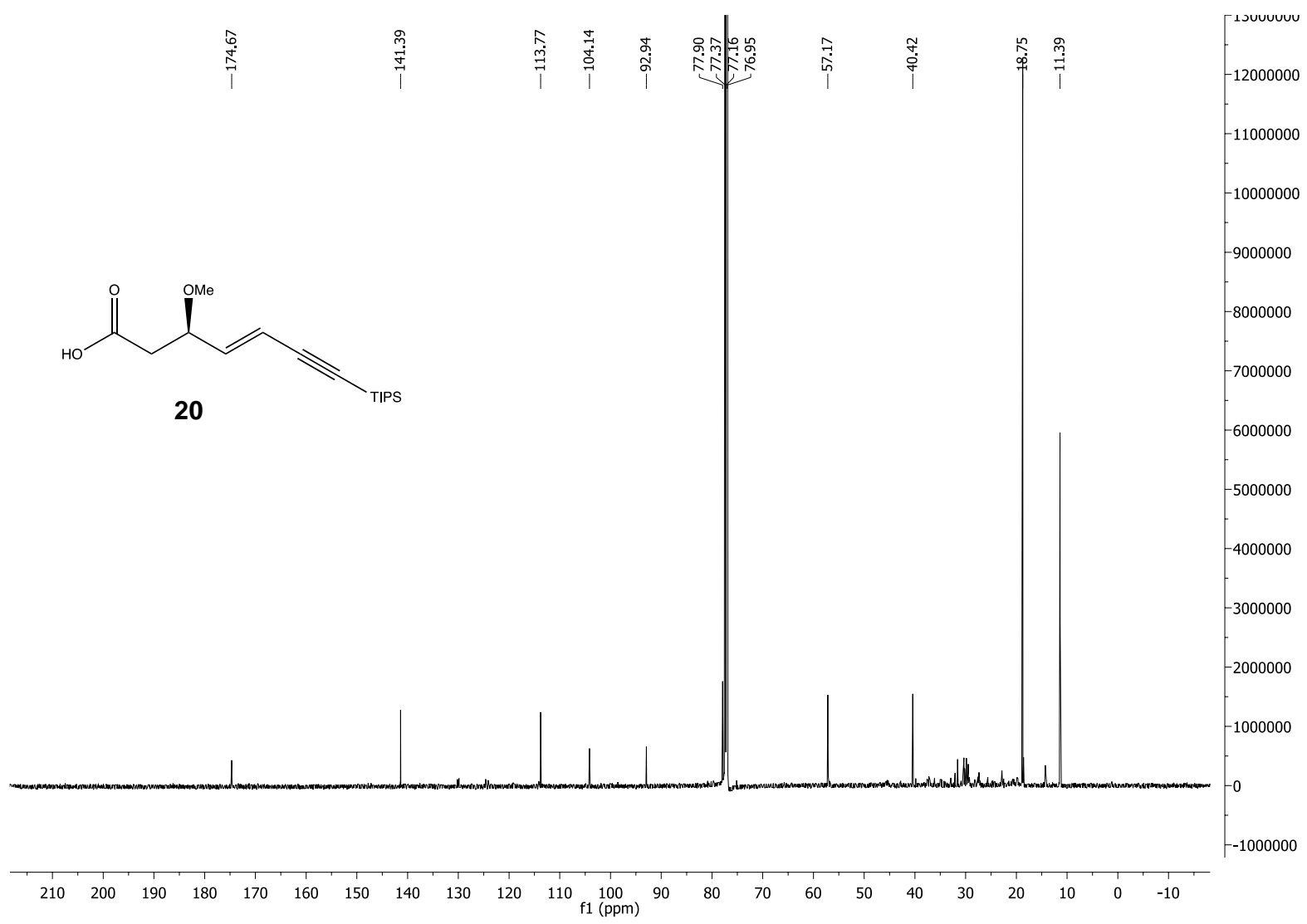


${ }^{1} \mathrm{H}$ NMR $\left(400 \mathrm{MHz}, \mathrm{CD}_{3} \mathrm{OD}\right)$

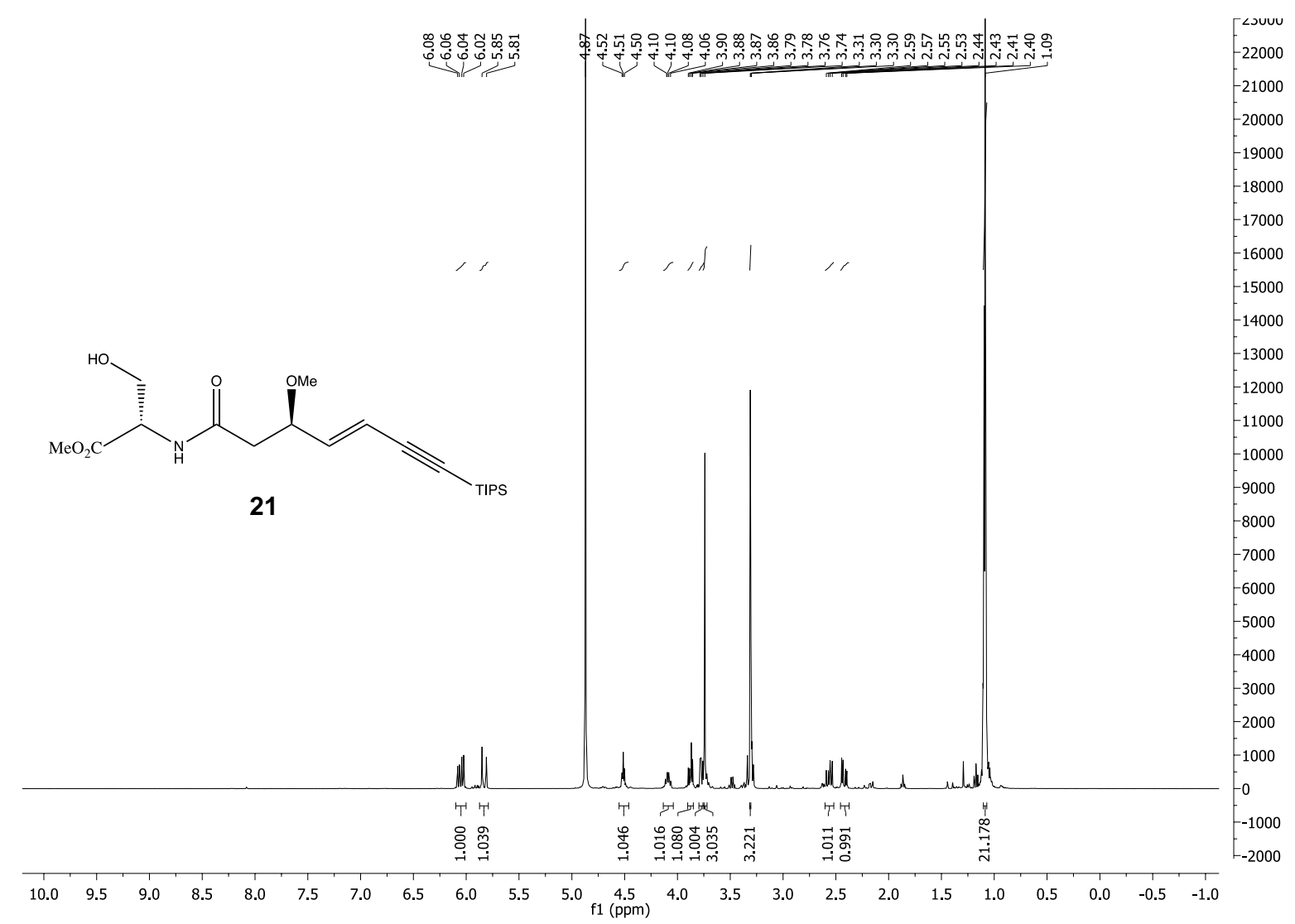

${ }^{13} \mathrm{C}$ NMR $\left(100 \mathrm{MHz}, \mathrm{CD}_{3} \mathrm{OD}\right)$

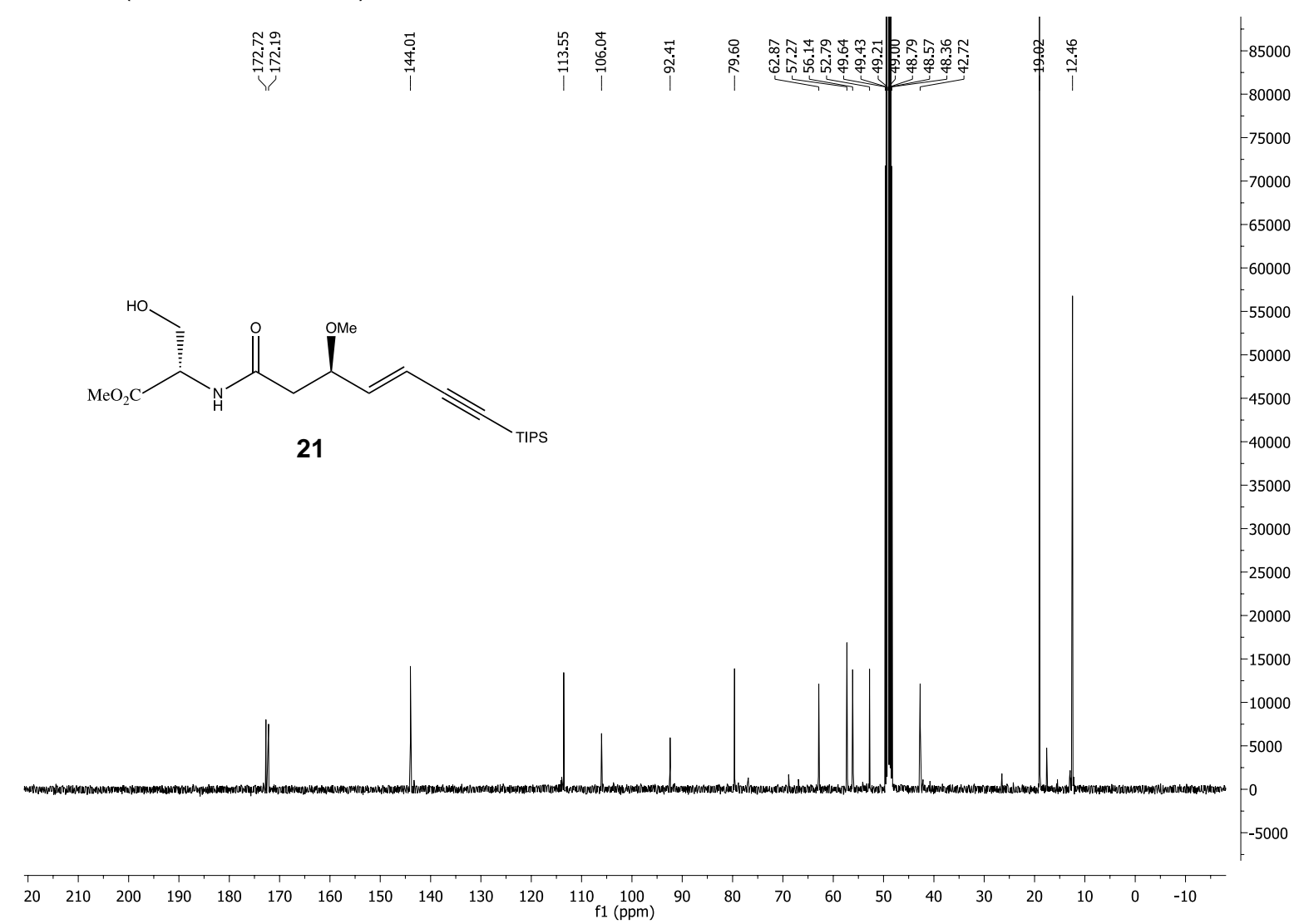


${ }^{1} \mathrm{H}$ NMR $\left(400 \mathrm{MHz}, \mathrm{CDCl}_{3}\right)$

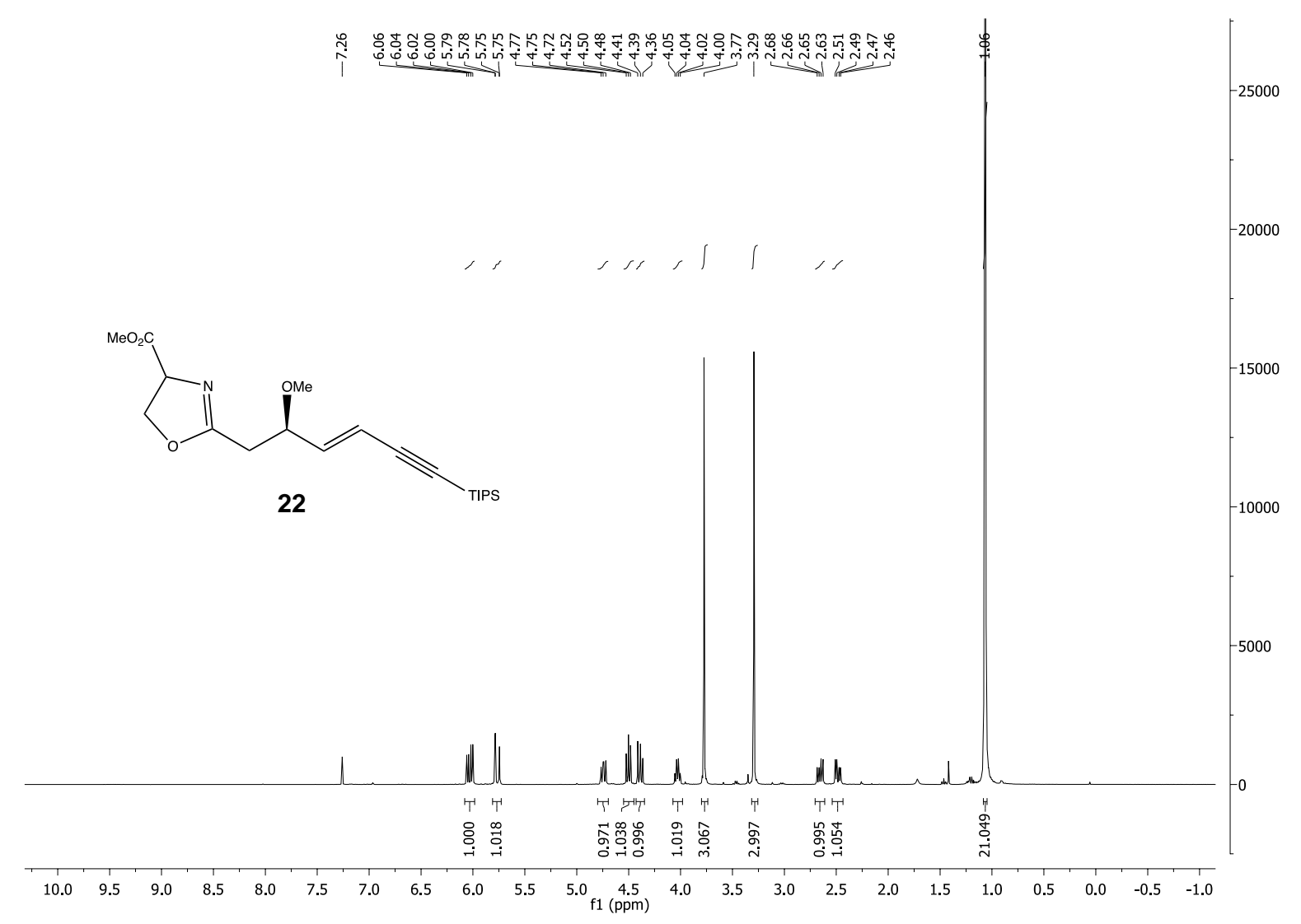

${ }^{13} \mathrm{C}$ NMR $\left(100 \mathrm{MHz}, \mathrm{CDCl}_{3}\right)$

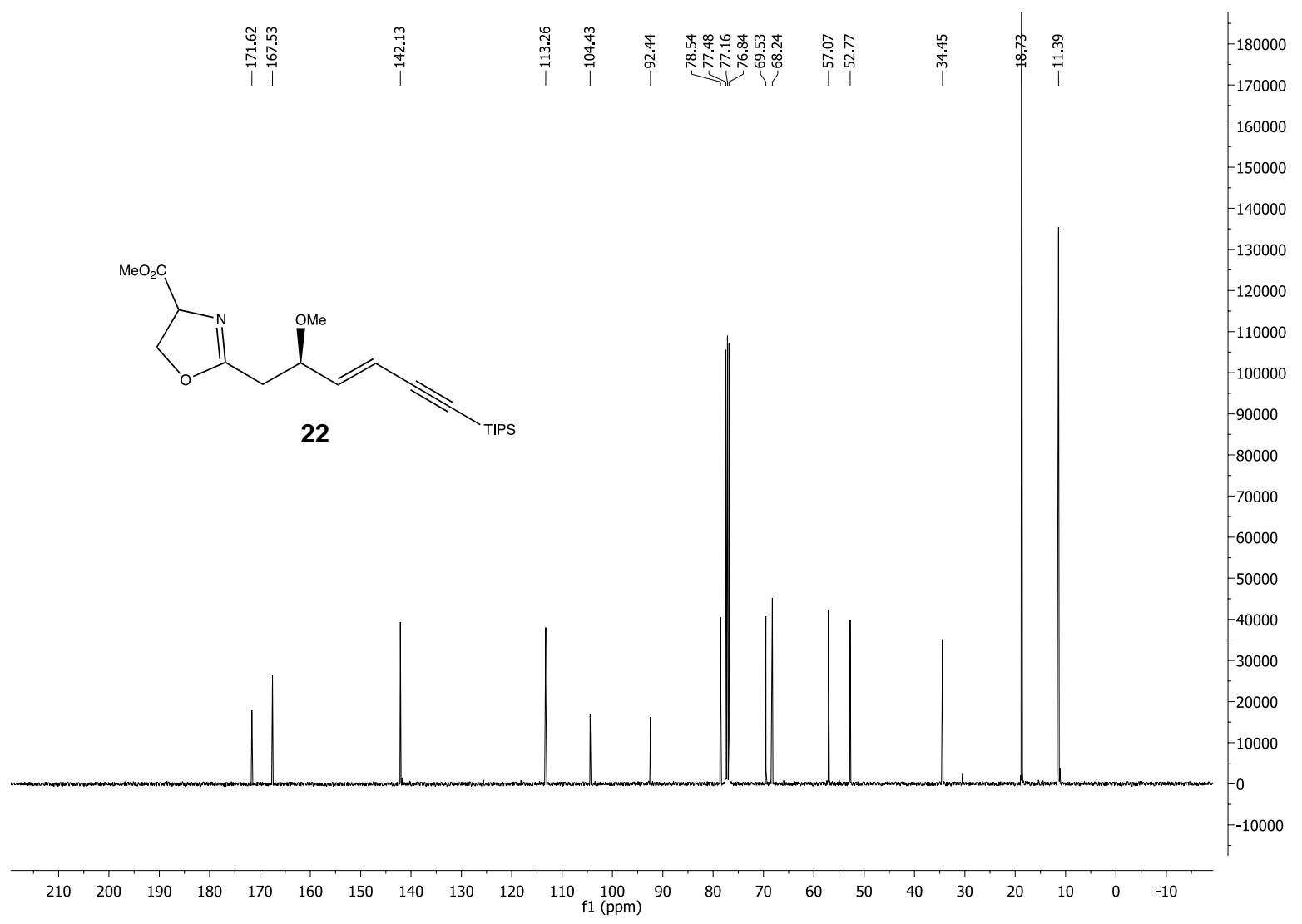


${ }^{1} \mathrm{H}$ NMR $\left(400 \mathrm{MHz}, \mathrm{CDCl}_{3}\right)$

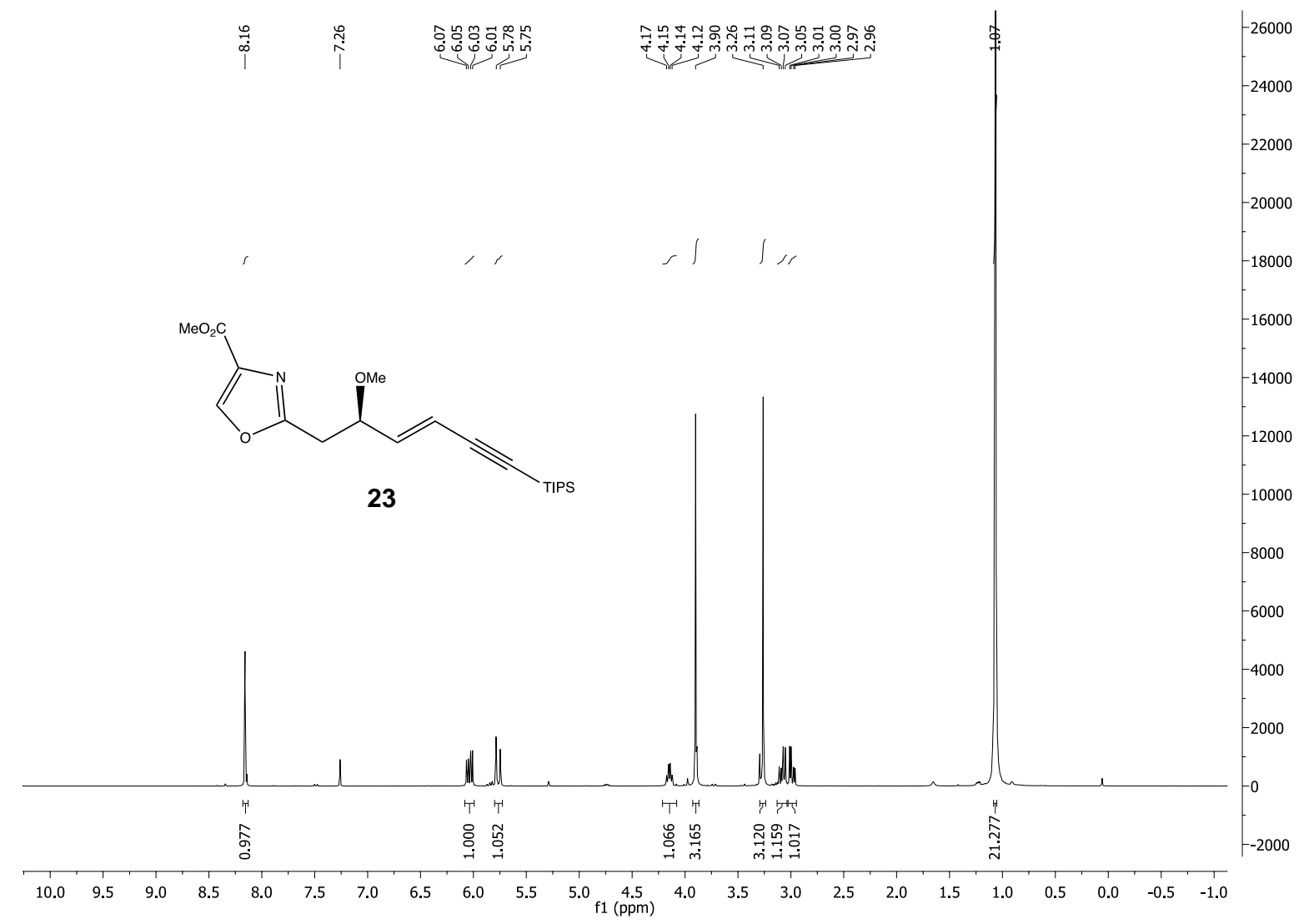

$\left.{ }^{13} \mathrm{C} \mathrm{NMR} \mathrm{(100} \mathrm{MHz,} \mathrm{CDCl}_{3}\right)$

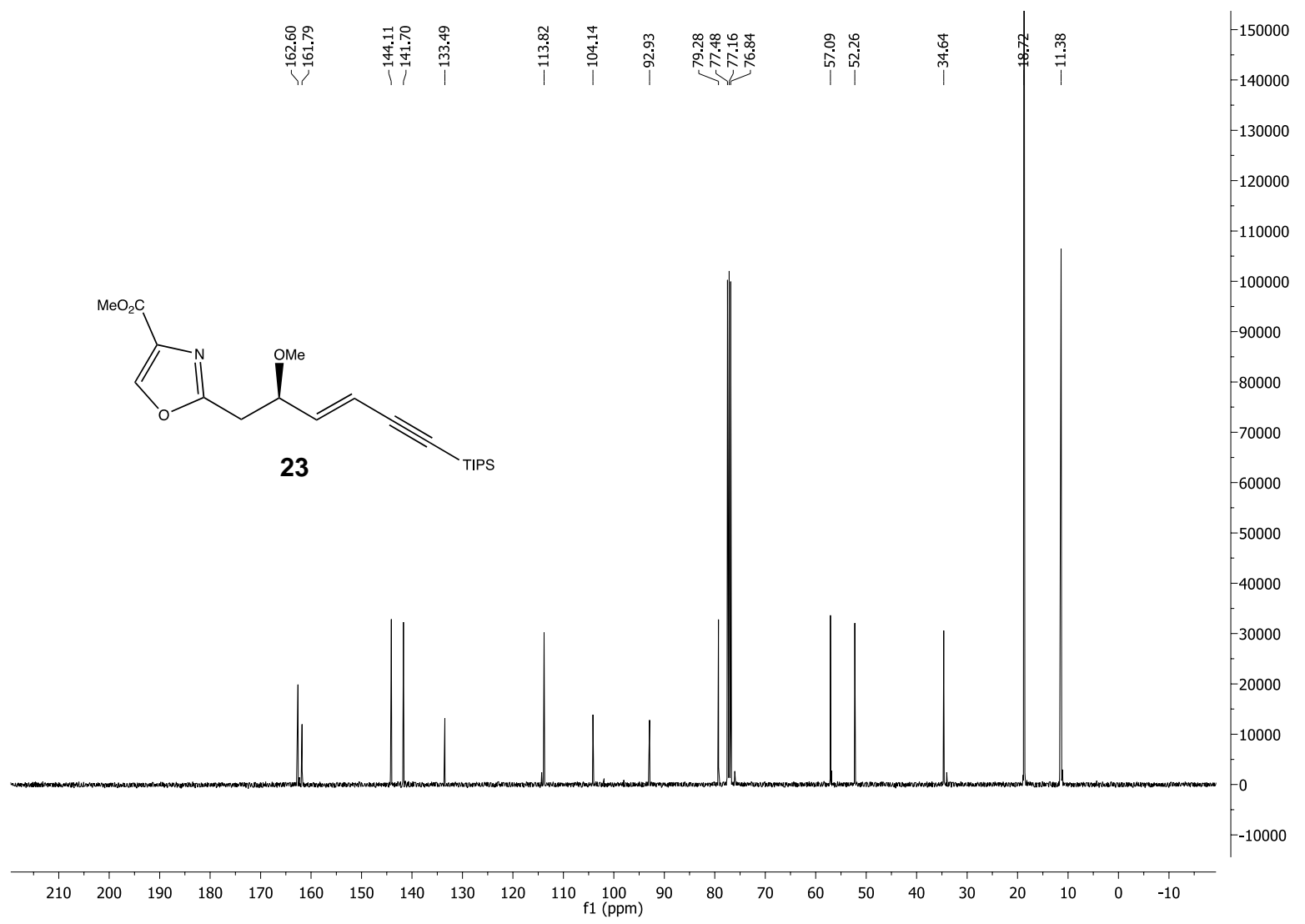


${ }^{1} \mathrm{H}$ NMR (400 MHz, $\left.\mathrm{CDCl}_{3}\right)$

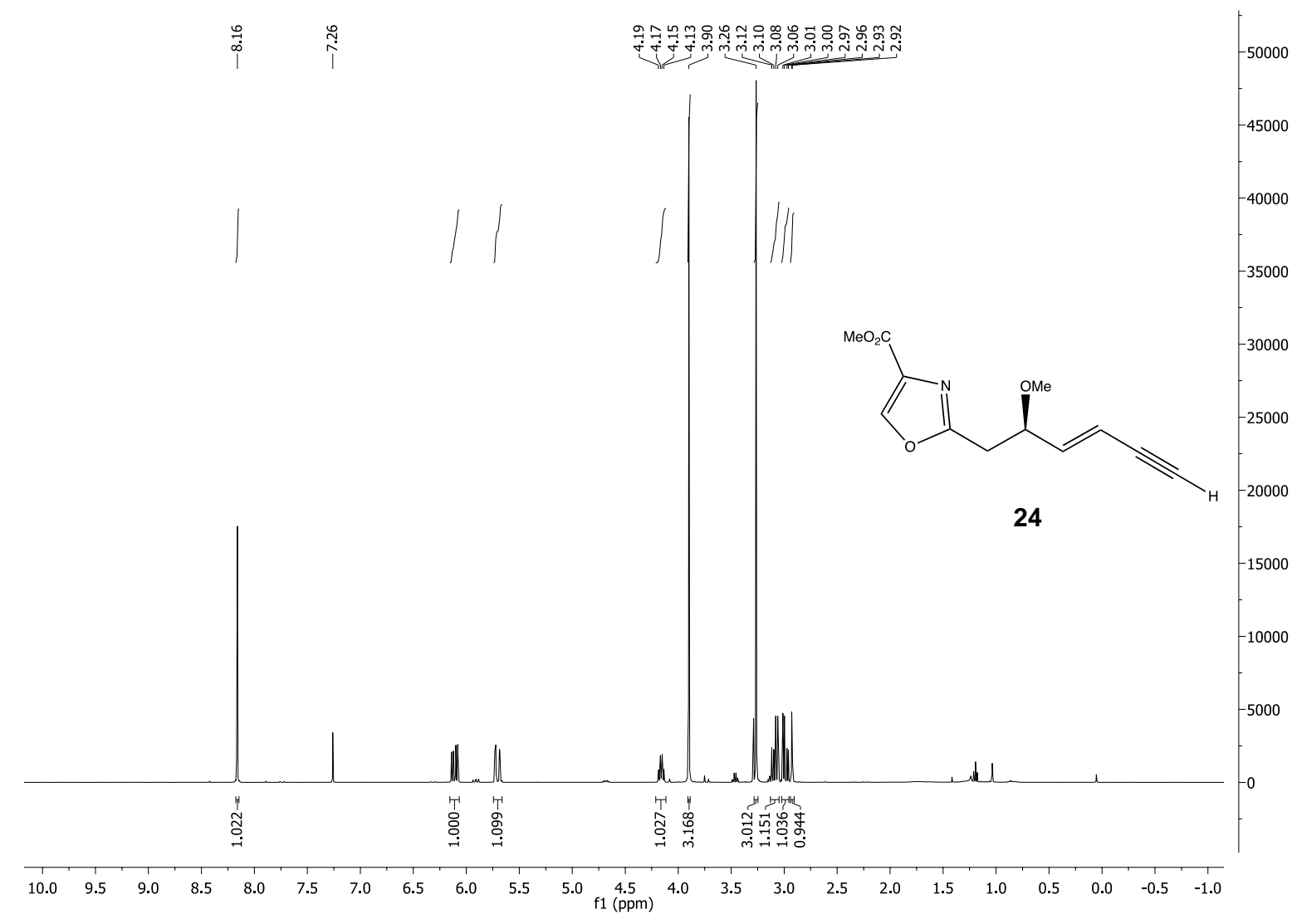

${ }^{13} \mathrm{C}$ NMR $\left(100 \mathrm{MHz}, \mathrm{CDCl}_{3}\right)$

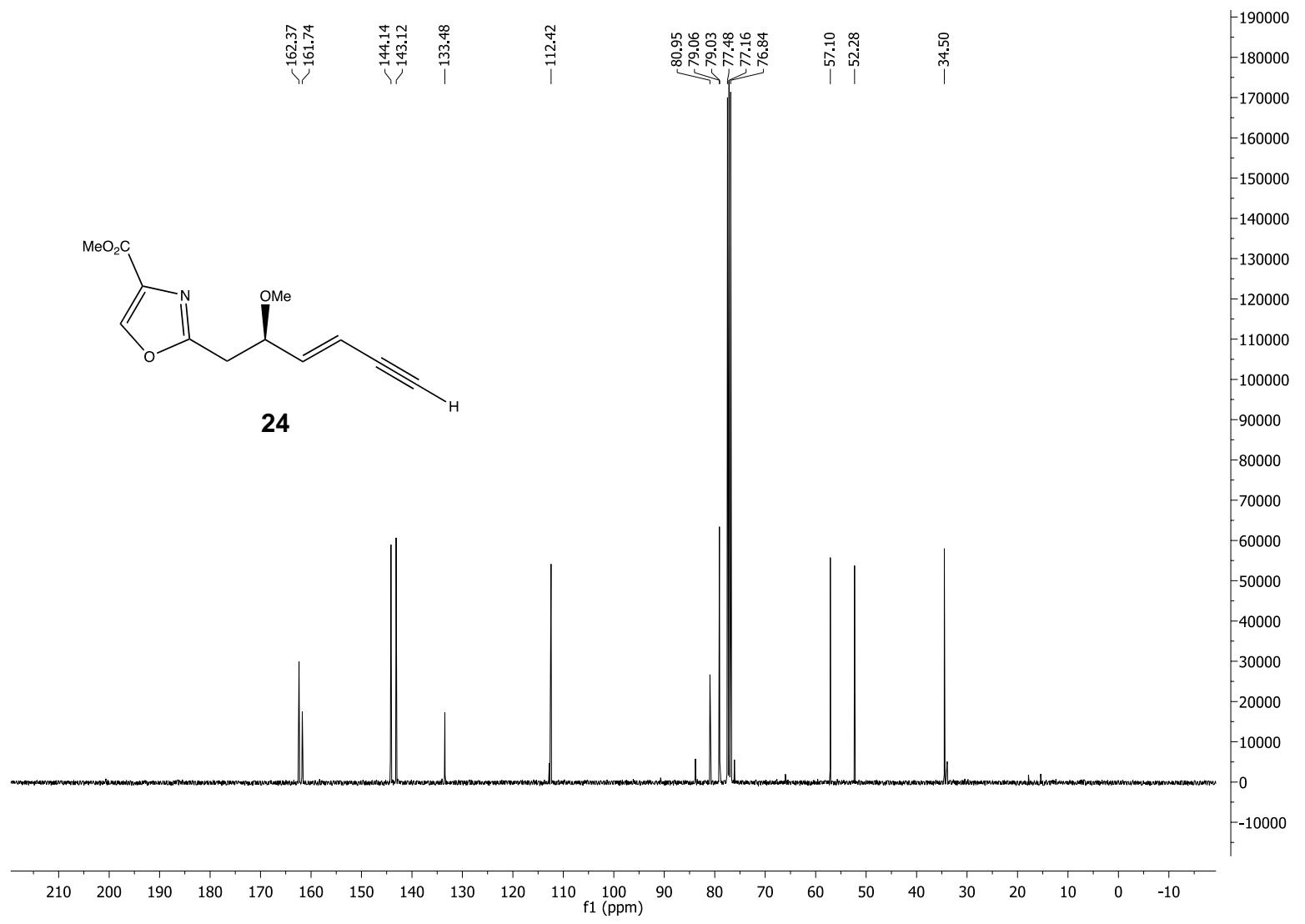


${ }^{1} \mathrm{H}$ NMR $\left(600 \mathrm{MHz}, \mathrm{CDCl}_{3}\right)$

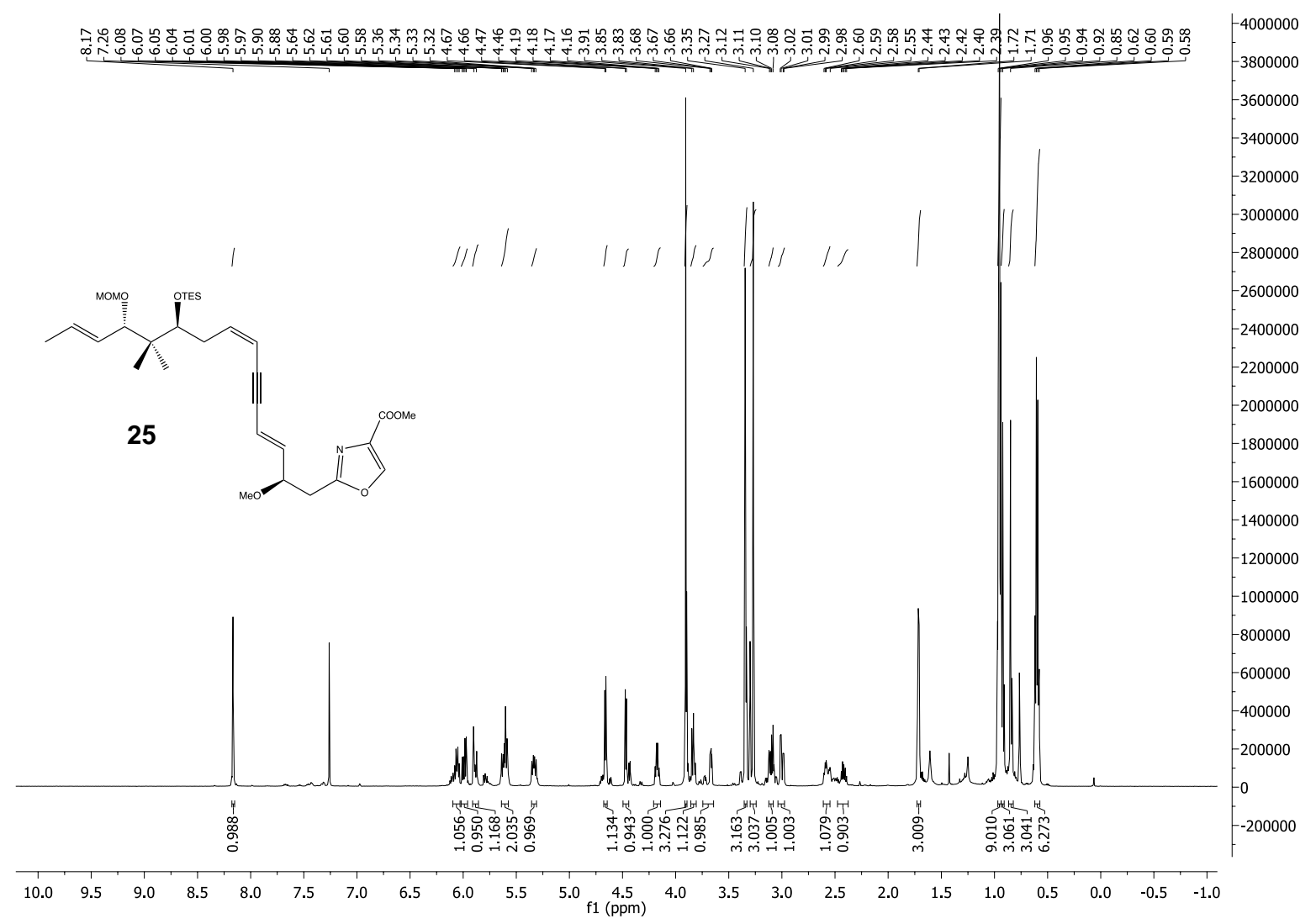

${ }^{13} \mathrm{C}$ NMR (151 MHz, $\left.\mathrm{CDCl}_{3}\right)$

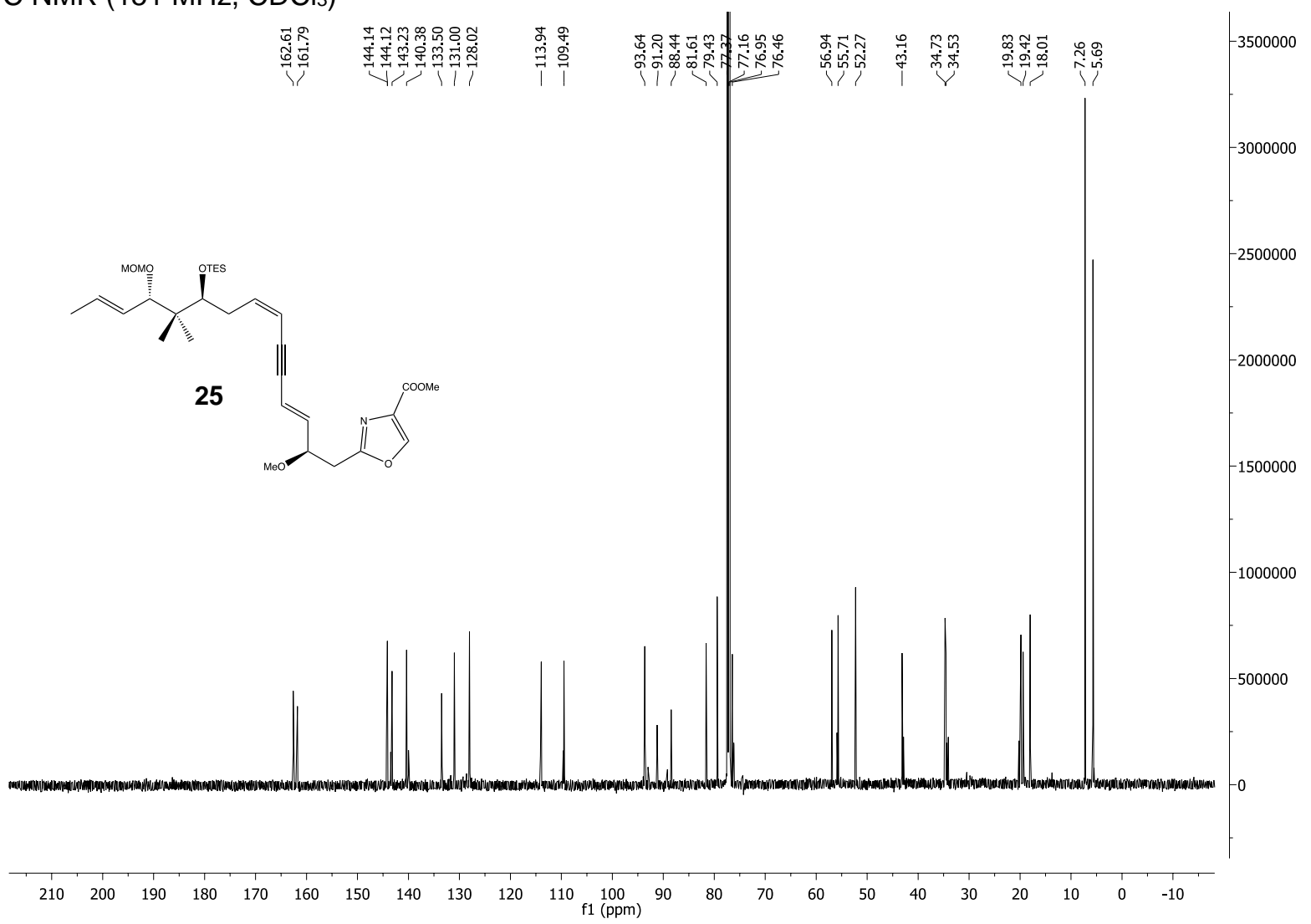


${ }^{1} \mathrm{H} \mathrm{NMR}\left(600 \mathrm{MHz}, \mathrm{CDCl}_{3}\right)$

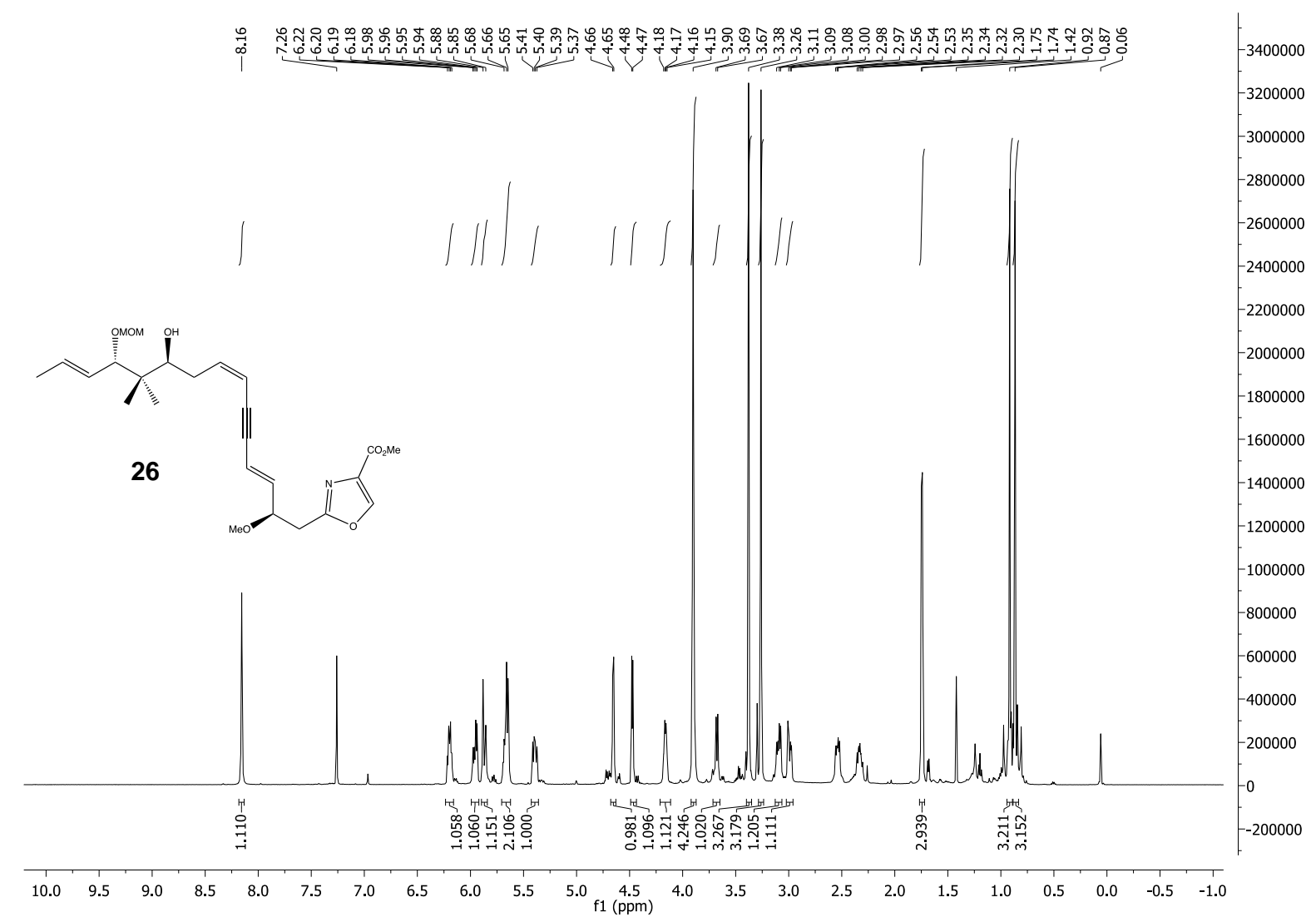

${ }^{13} \mathrm{C} \mathrm{NMR}\left(151 \mathrm{MHz}, \mathrm{CDCl}_{3}\right)$

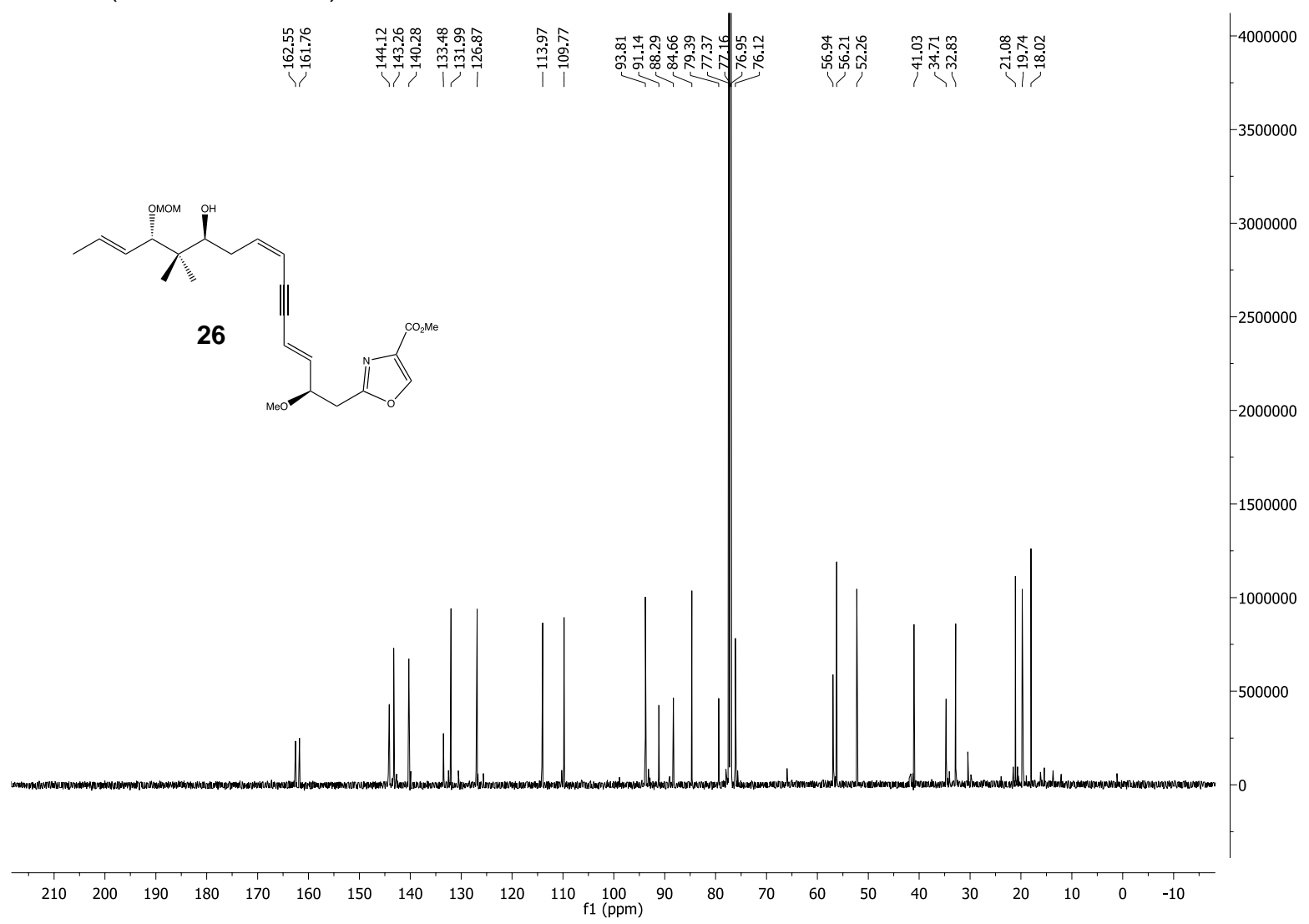


${ }^{1} \mathrm{H}$ NMR $\left(600 \mathrm{MHz}, \mathrm{CDCl}_{3}\right)$

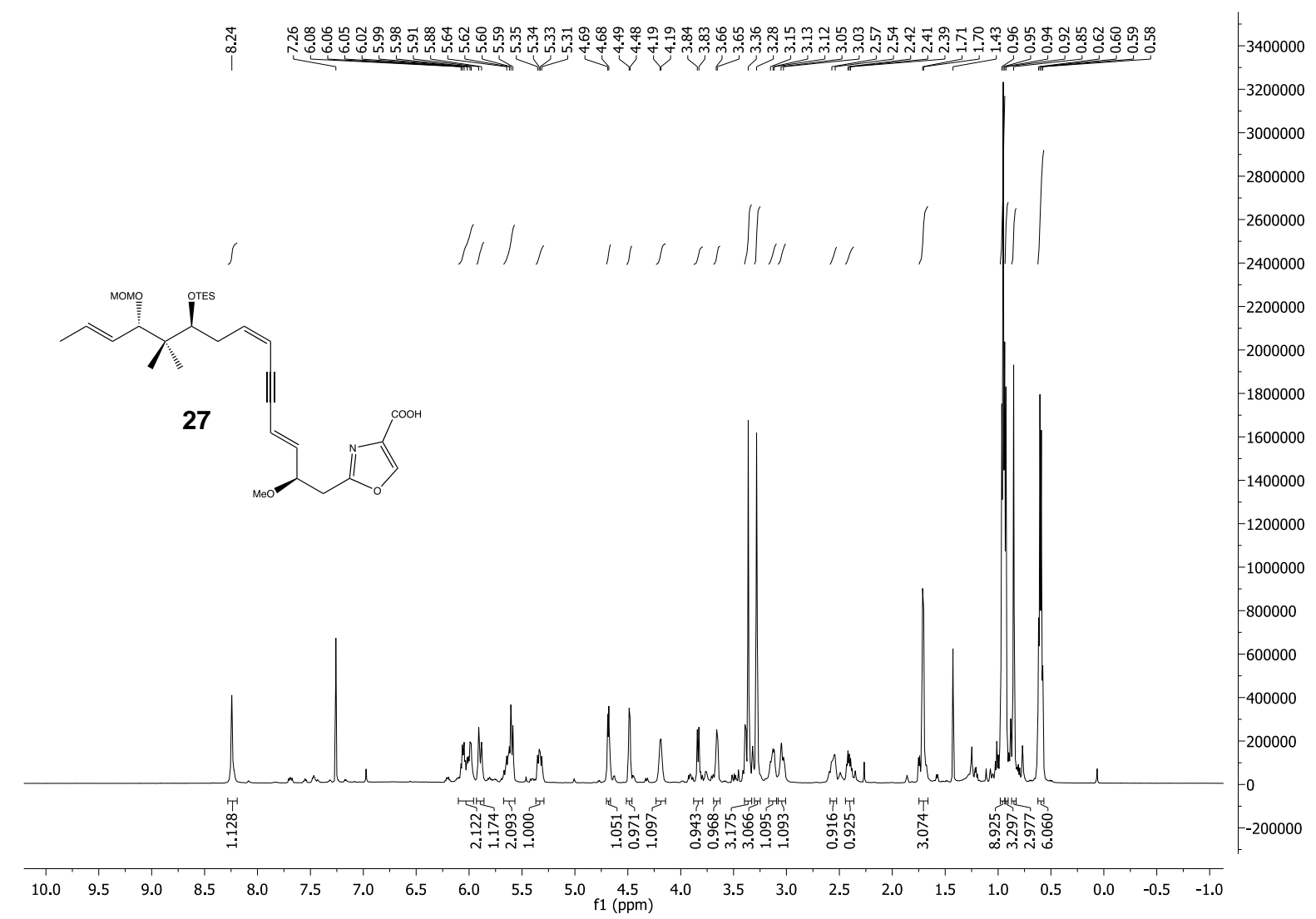

${ }^{13} \mathrm{C}$ NMR $\left(151 \mathrm{MHz}, \mathrm{CDCl}_{3}\right)$

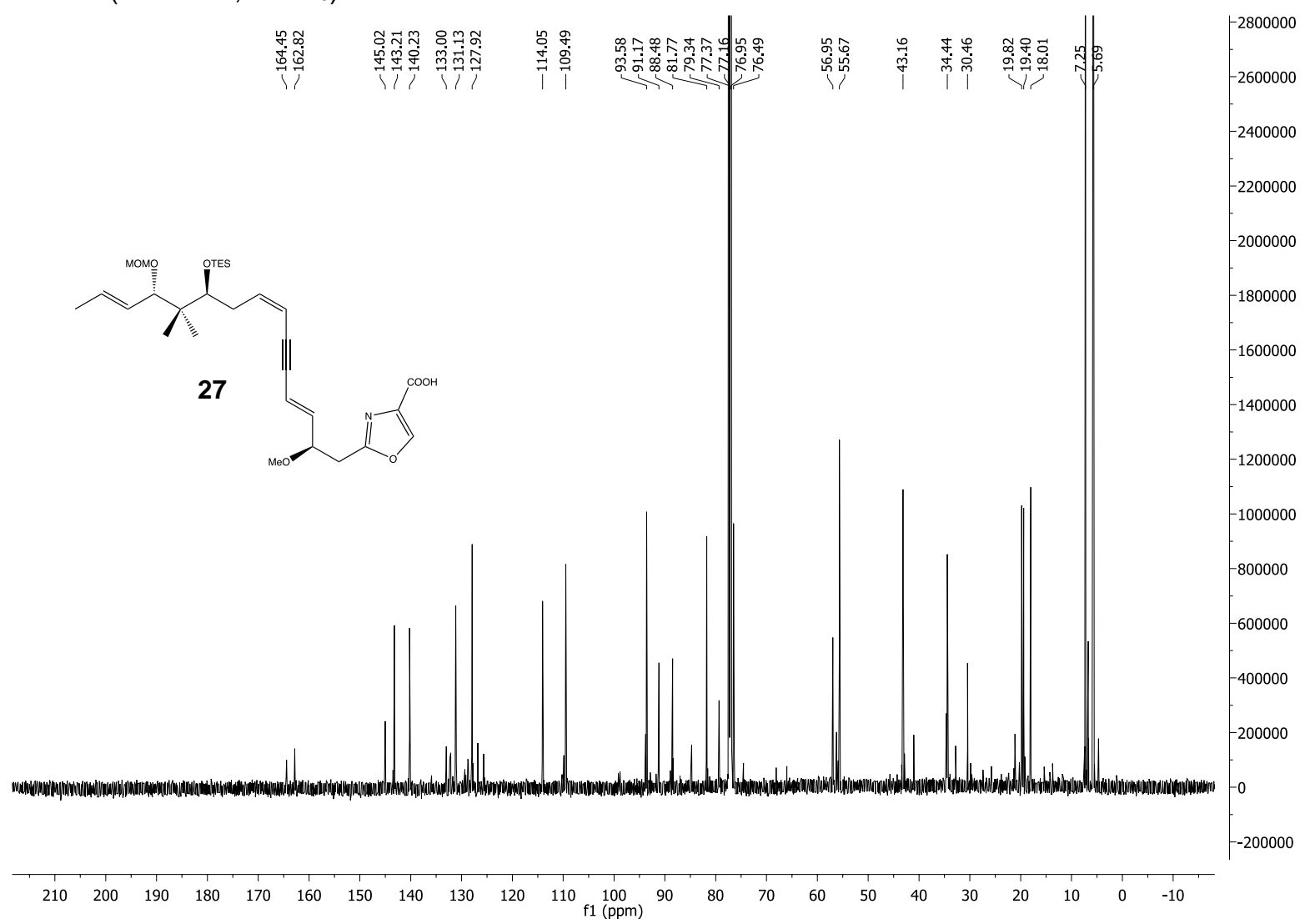




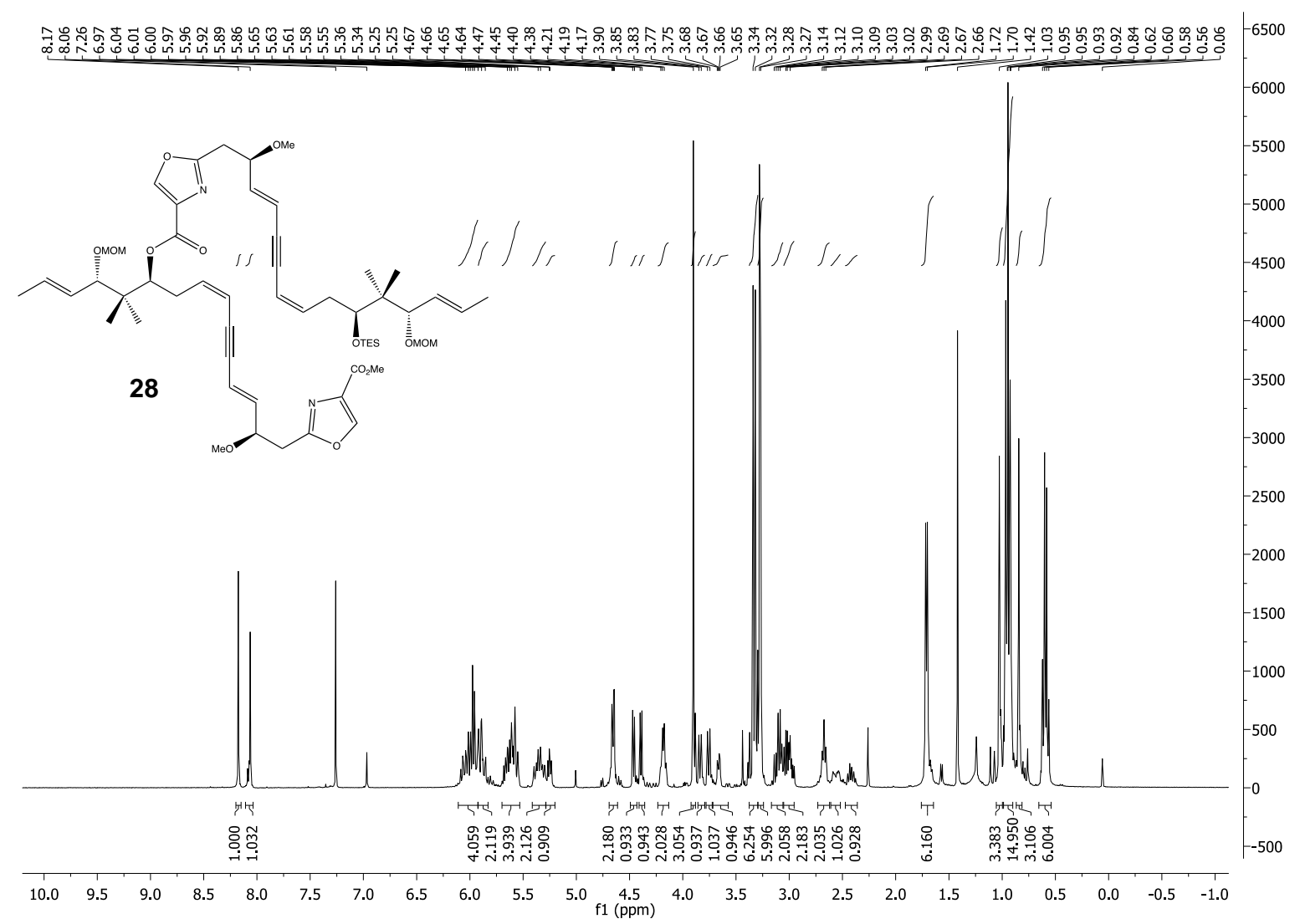

${ }^{13} \mathrm{C}$ NMR $\left(100 \mathrm{MHz}, \mathrm{CDCl}_{3}\right)$

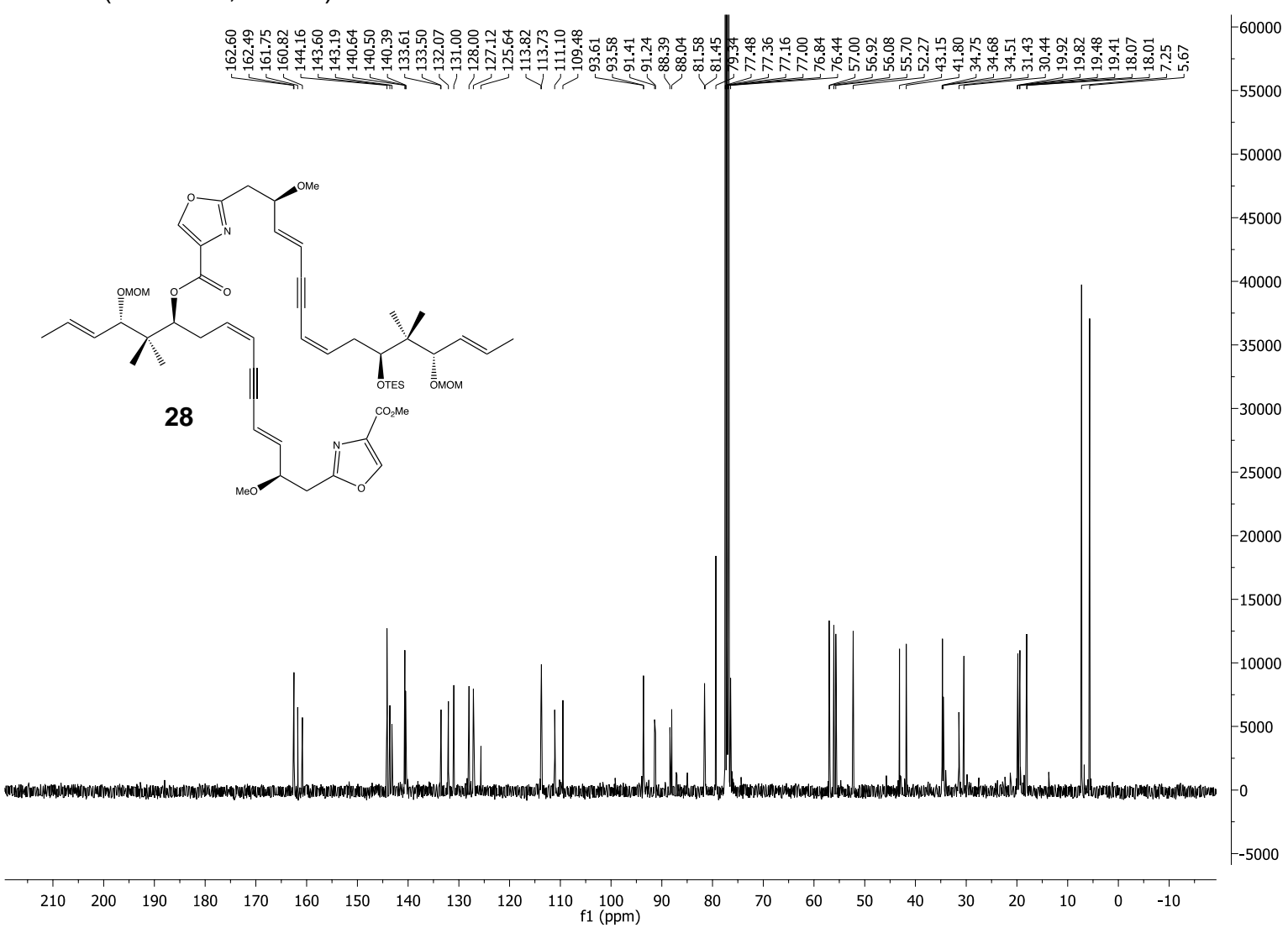




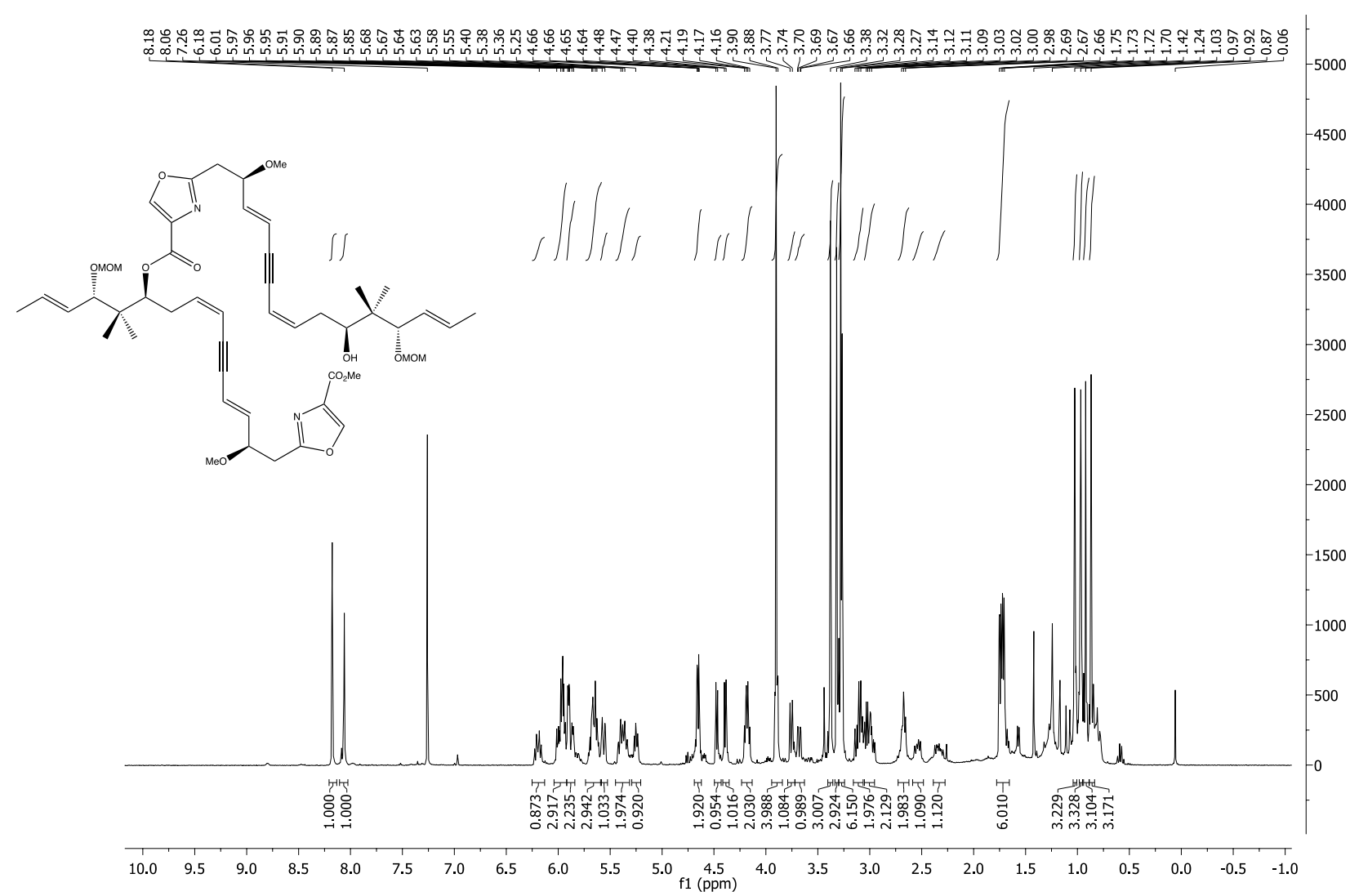

${ }^{13} \mathrm{C}$ NMR $\left(100 \mathrm{MHz}, \mathrm{CDCl}_{3}\right)$

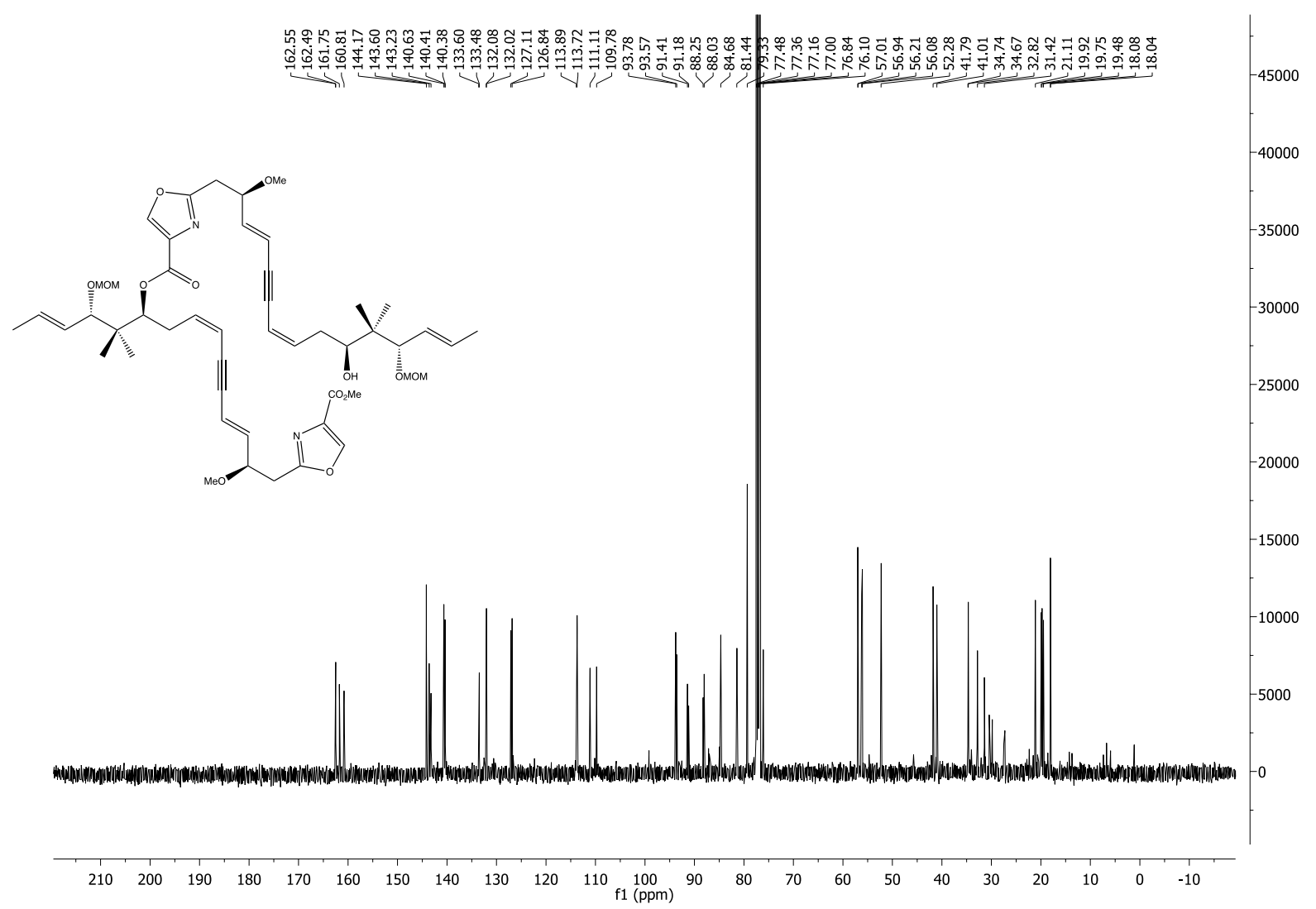




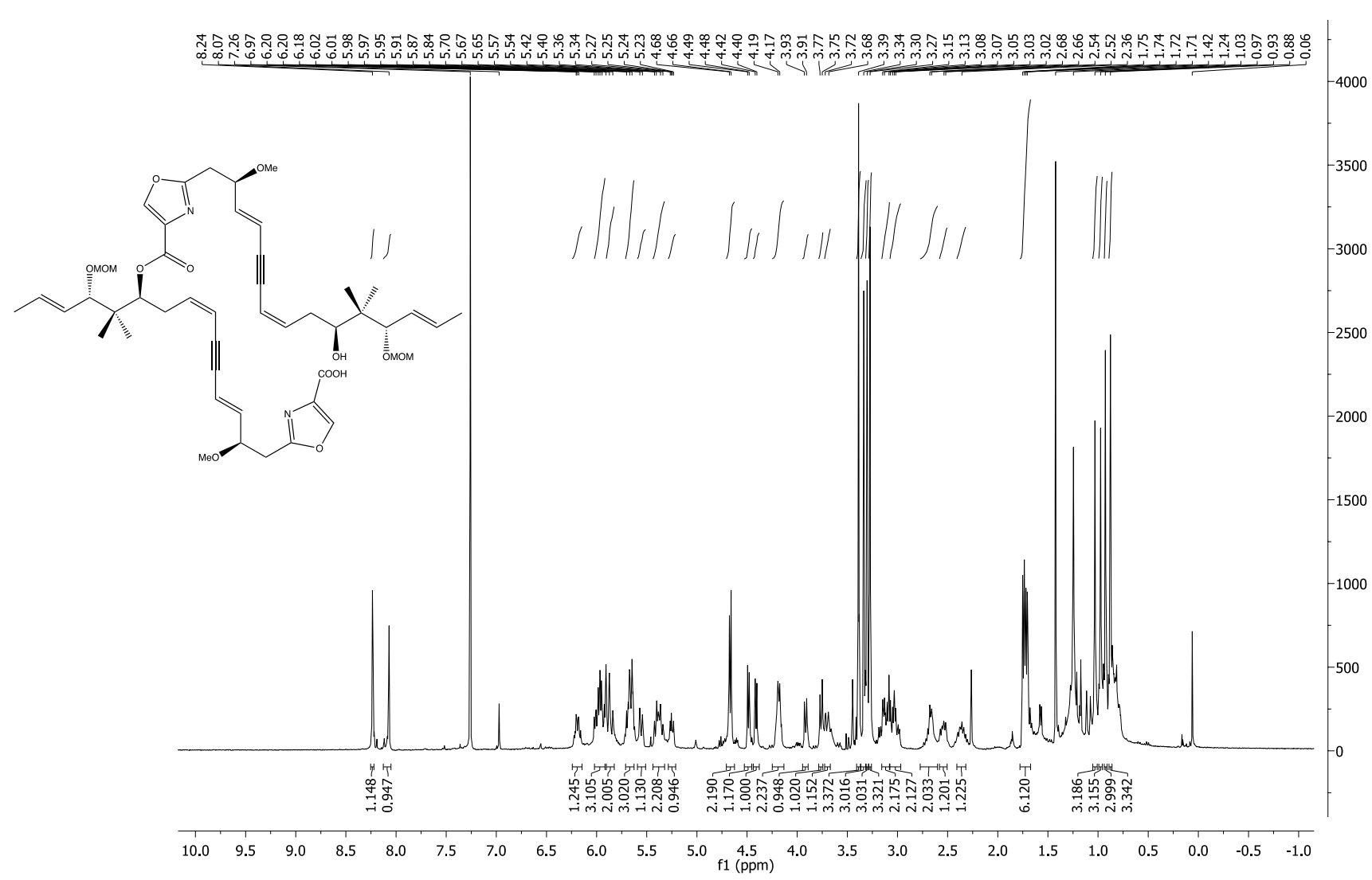

${ }^{13} \mathrm{C} \mathrm{NMR}\left(100 \mathrm{MHz}, \mathrm{CDCl}_{3}\right)$

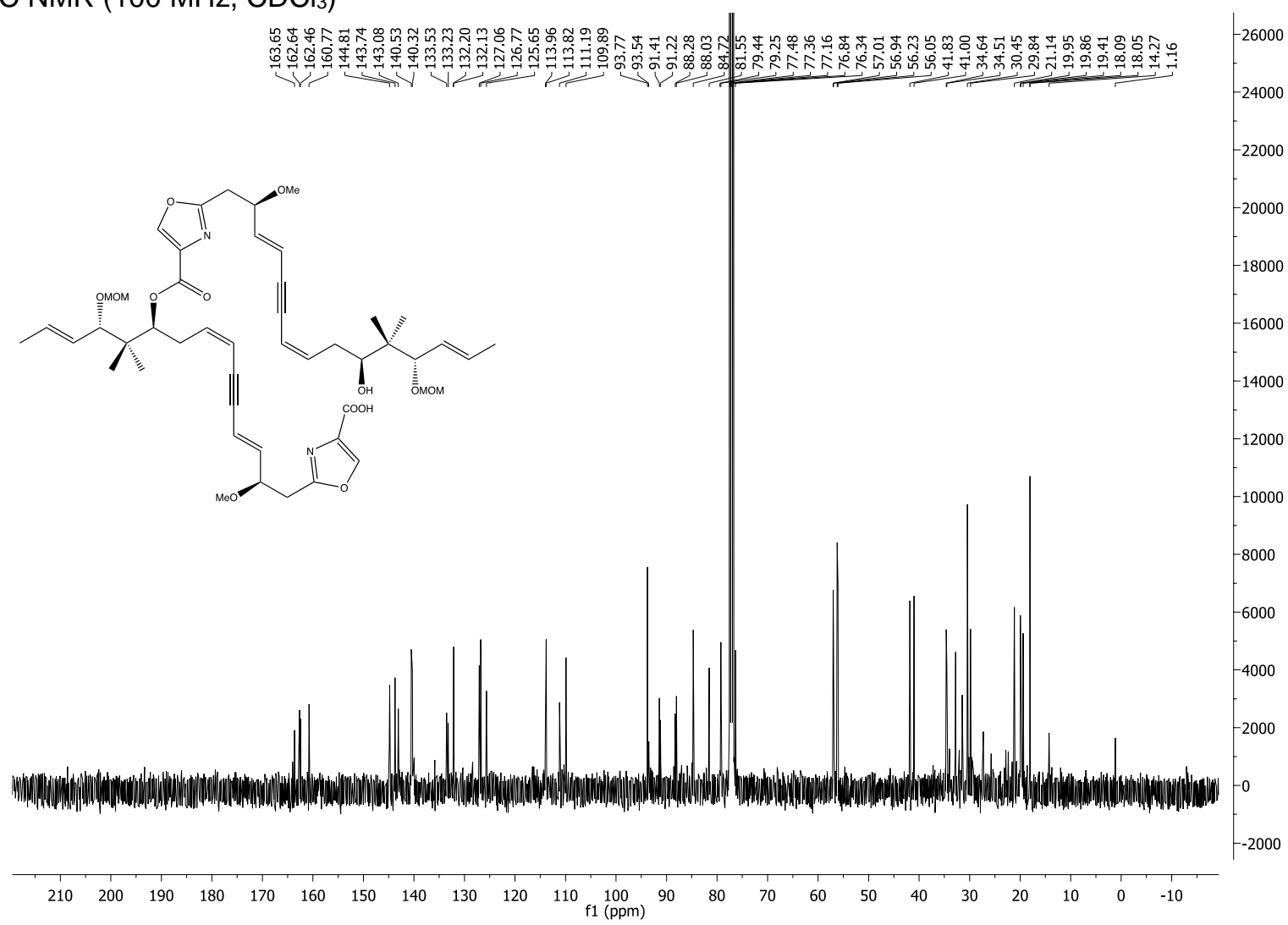




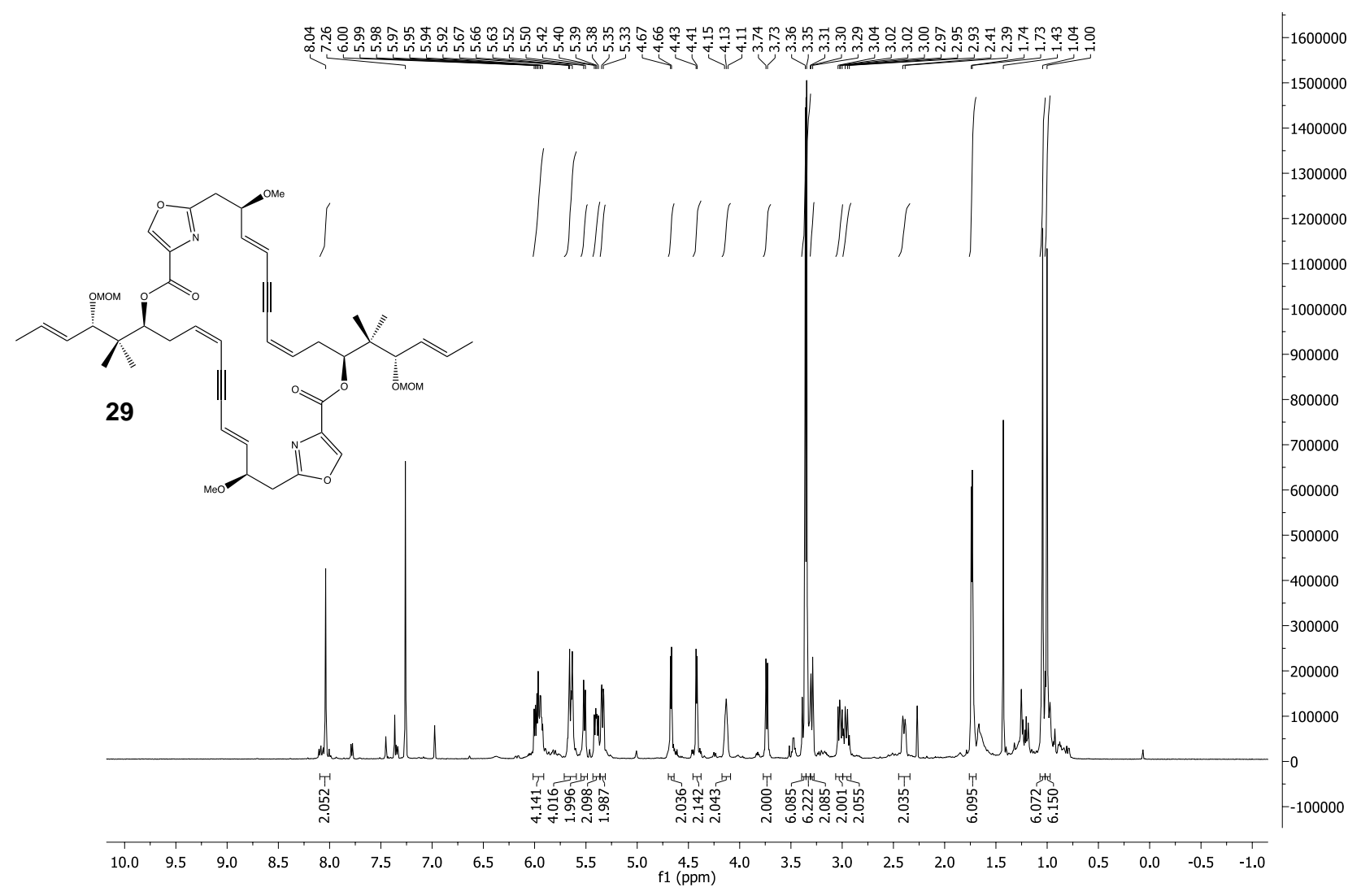

${ }^{13} \mathrm{C} \mathrm{NMR} \mathrm{(151} \mathrm{MHz,} \mathrm{CDCl}_{3}$ )

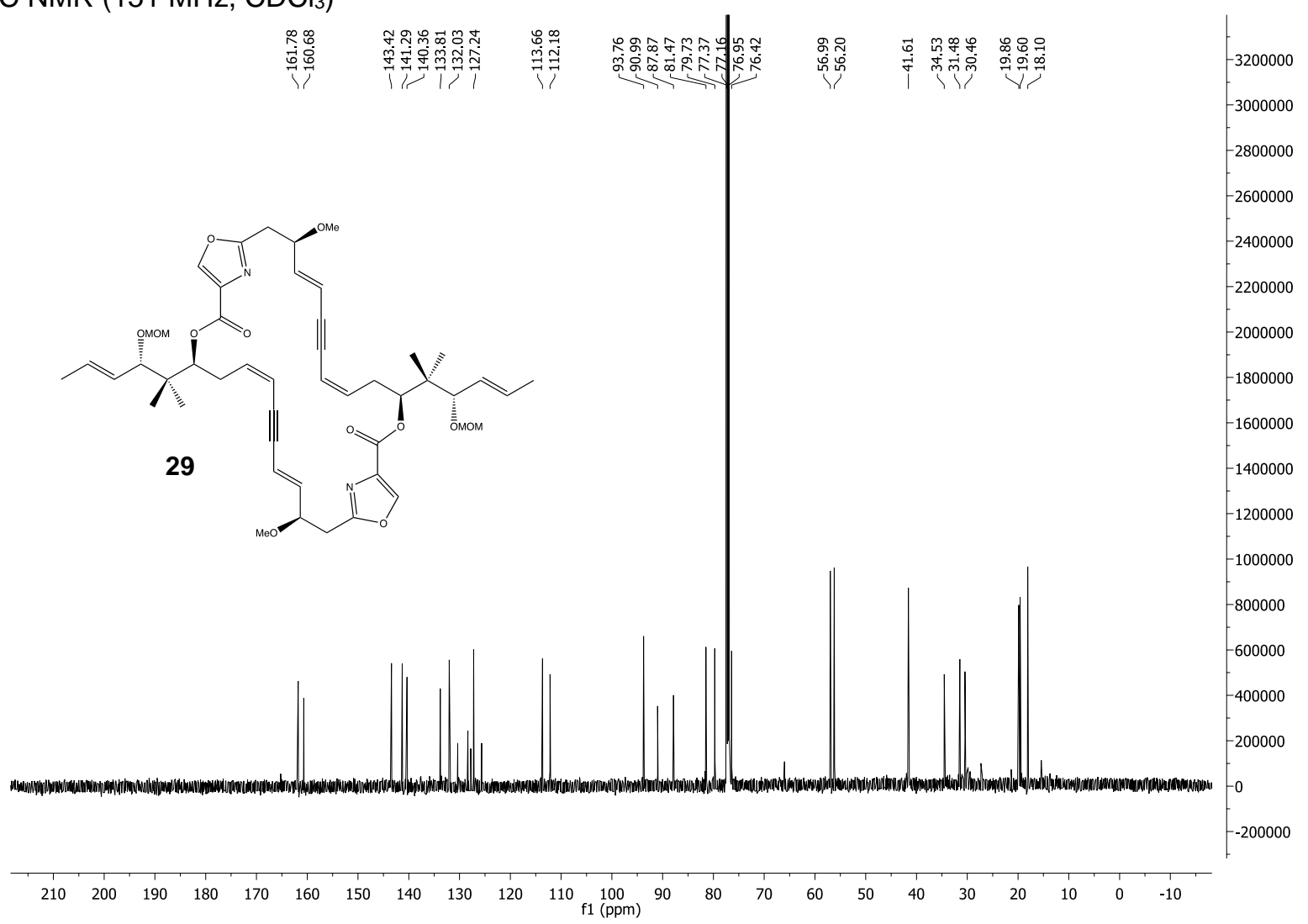


${ }^{1} \mathrm{H} \mathrm{NMR}\left(600 \mathrm{MHz}, \mathrm{CDCl}_{3}\right)$

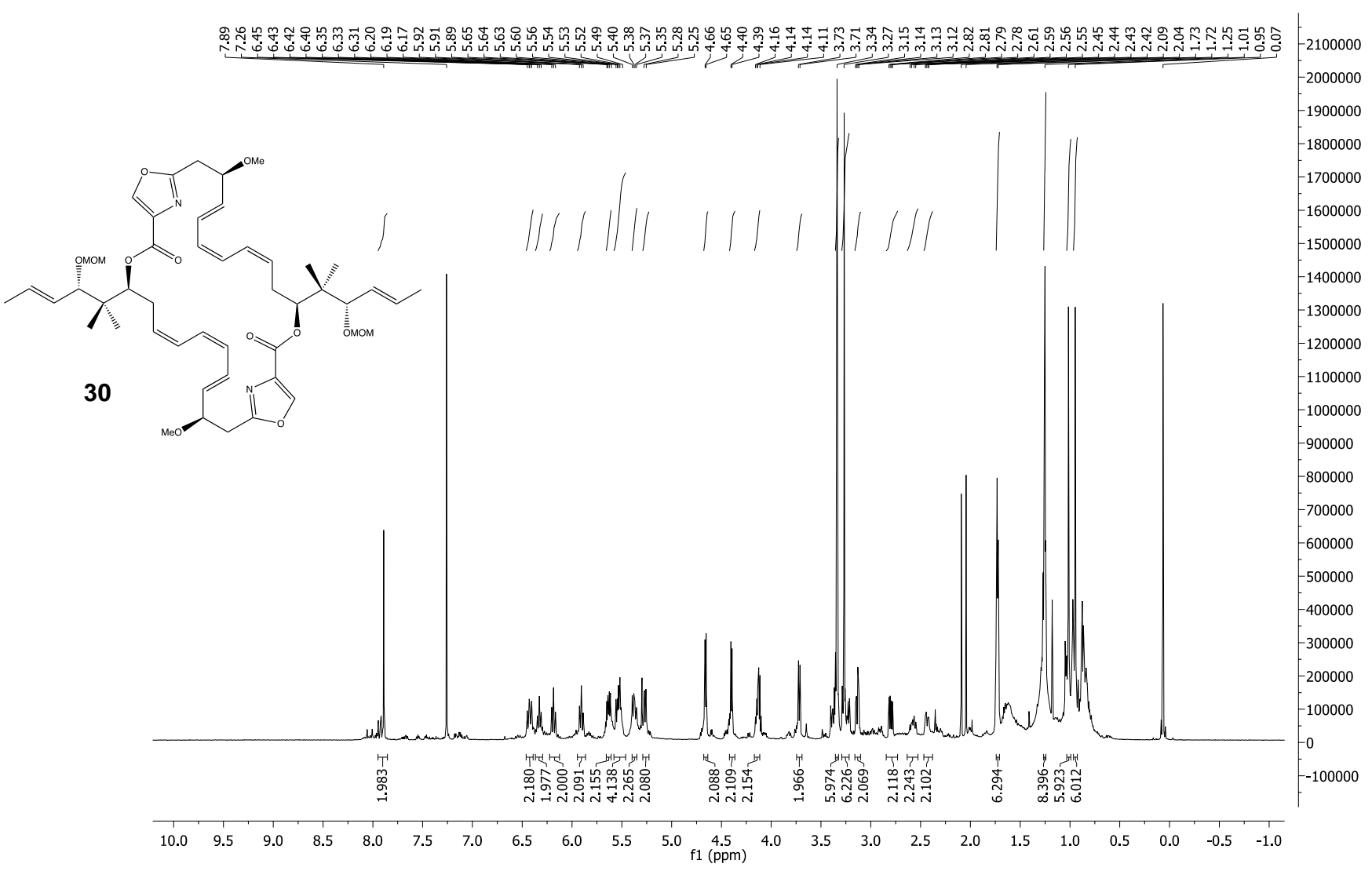

${ }^{13} \mathrm{C} \mathrm{NMR} \mathrm{(151} \mathrm{MHz,} \mathrm{CDCl}_{3}$ )

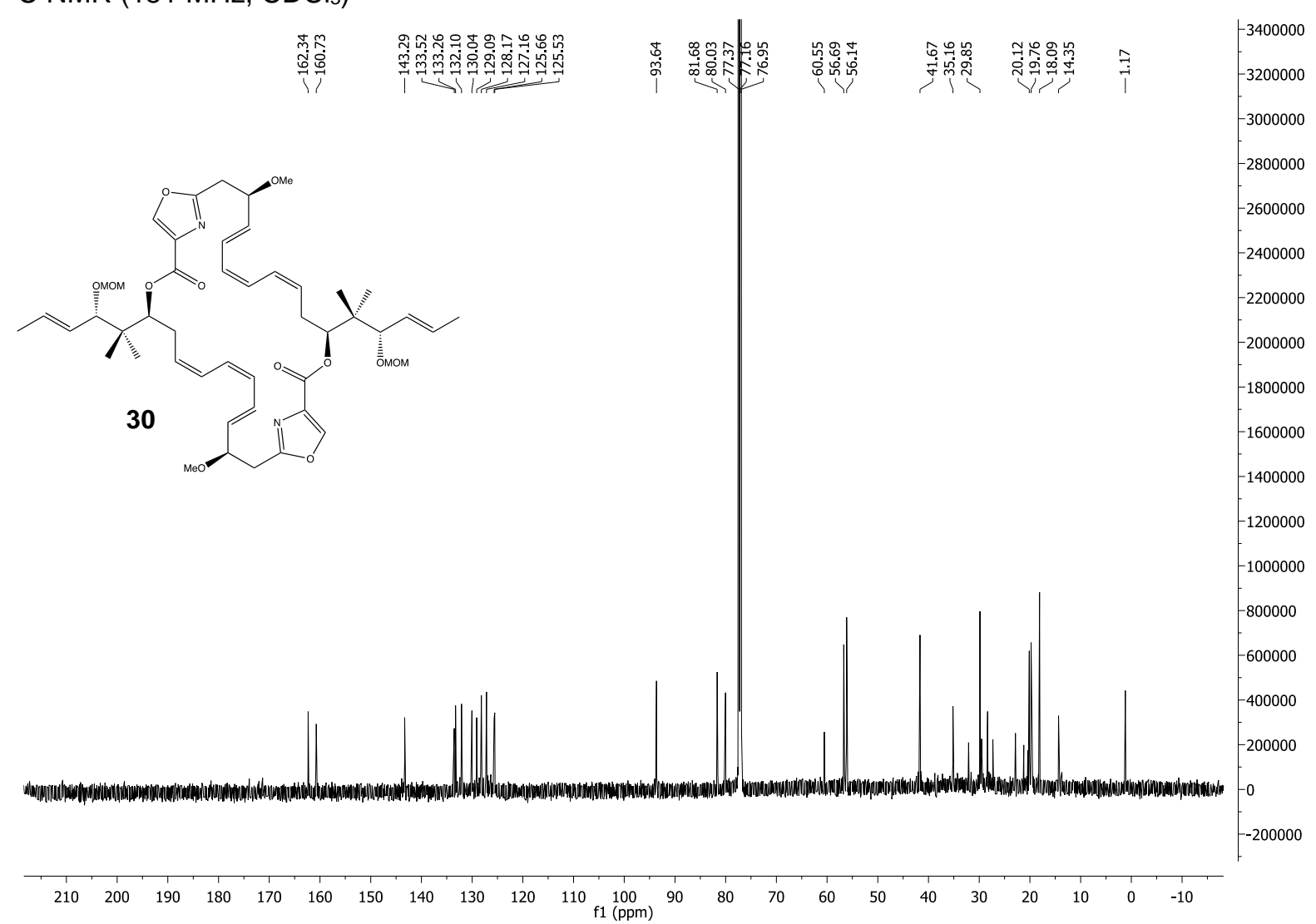


${ }^{1} \mathrm{H}$ NMR $\left(600 \mathrm{MHz}, \mathrm{CD}_{3} \mathrm{OD}\right)$

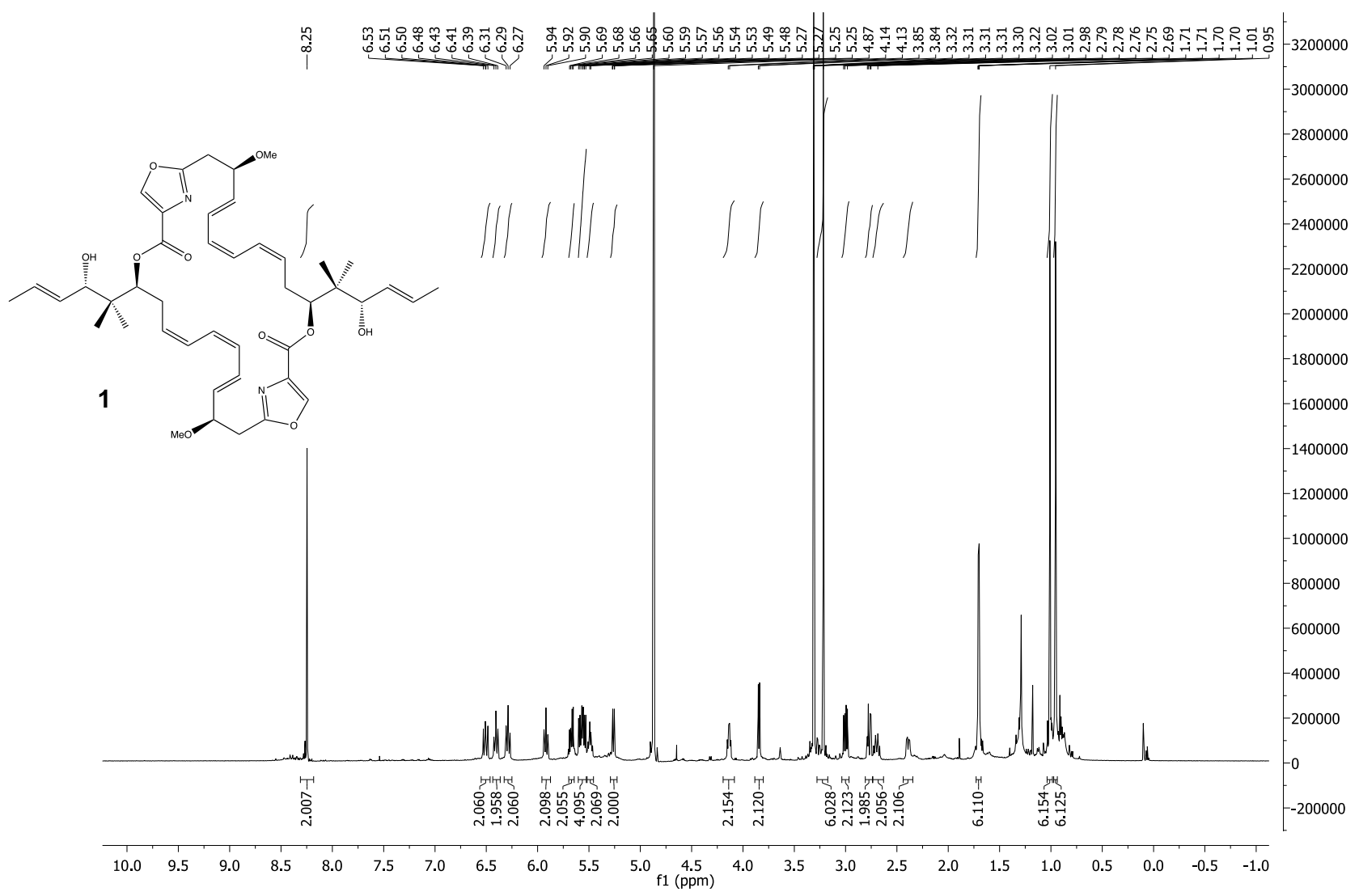

${ }^{13} \mathrm{C}$ NMR $\left(151 \mathrm{MHz}, \mathrm{CD}_{3} \mathrm{OD}\right)$

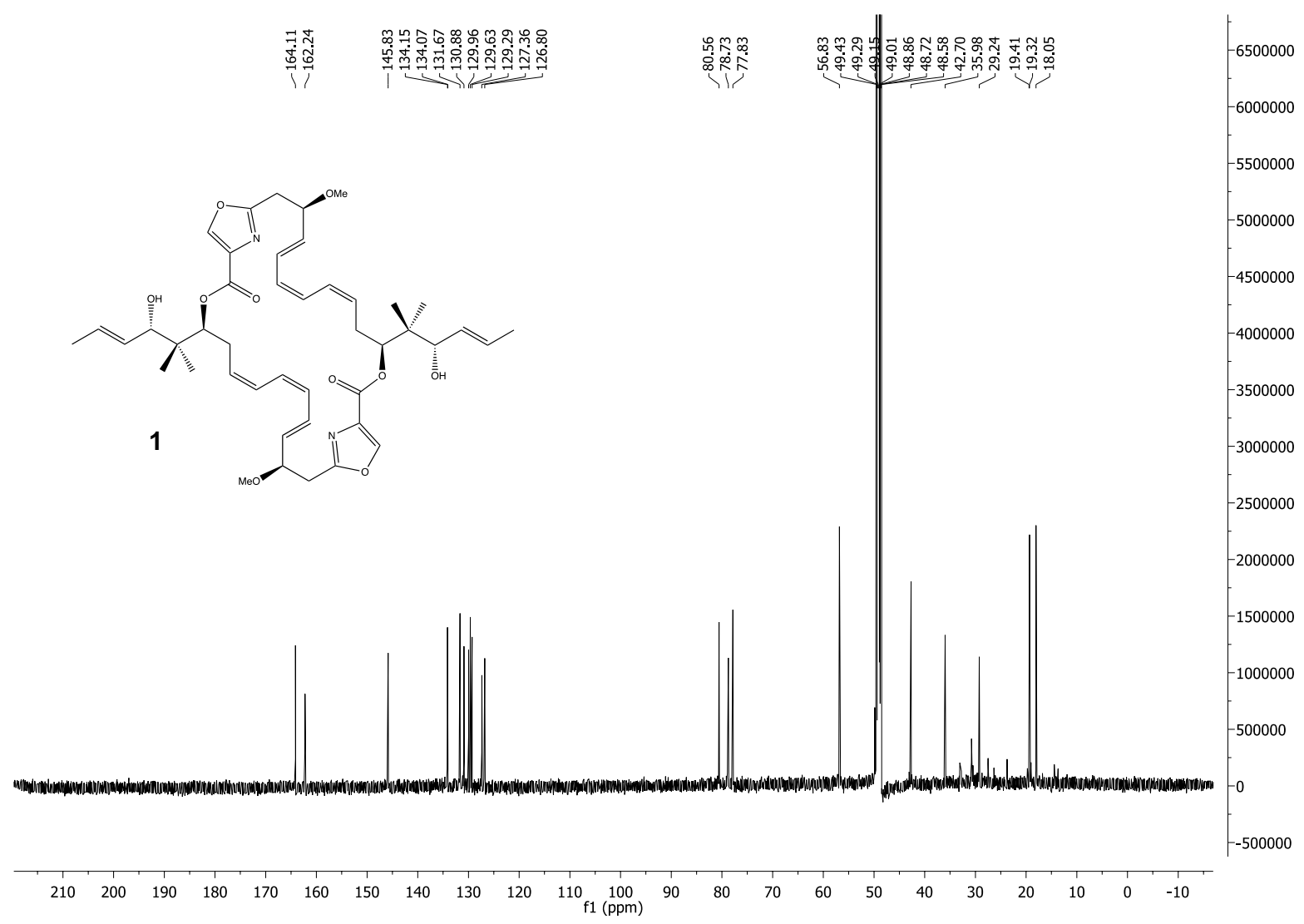

